\title{
Case Examination of Teacher-Tailored Student \\ Evaluation of Teaching Augmented with Peer \\ Observation of Teaching in a Vietnamese University
}

\author{
by \\ Nguyen Vu Phuong
}

\begin{abstract}
A thesis
submitted to the Victoria University of Wellington in fulfilment of the requirements for the degree of

Doctor of Philosophy in Education
\end{abstract}

Victoria University of Wellington 



\begin{abstract}
This study explored how teacher-tailored student evaluation of teaching (TT-SET) augmented with peer observation of teaching (POT) impacted on academics' pedagogical reasoning in a Vietnamese university. The qualitative case study within the constructivist paradigm used multiple data collection methods. Detailed analysis developed findings through descriptions of individual academics' experience of the intervention and analysis across participants (using thematic analysis).

The intervention encouraged academics to reflect on their practice. This reflection promoted changes in their understandings of practice, actions to refine practice, future plans, and other outcomes (e.g., enhanced confidence, self-efficacy, sense of autonomy, and collegiality). However, there were several challenges which varied among the participants, including perceiving TT-SET as lacking reliability and validity, limited learning from junior peers, disagreement with feedback and lack of sensitivity, and limited time for POT and changes. The findings also suggested that among other factors, the nature of the peer relationship, which is under the impact of the Vietnamese Confucian collectivist culture, was important to successful implementation of the intervention. The theoretical framework developed for this study helps explain the changes in academics' pedagogical reasoning, particularly reflection.

The study contributes to the area of tertiary teacher development, both theoretically and practically. It offers insights into how such an approach may be effective, particularly in the context of Vietnamese higher education, and provides guidance for both practice and policies. It identifies what needs to be done to improve the implementation of the intervention. It also offers ideas for leaders to make institutional policies to support academics' professional learning and development. Its findings contribute to understanding how the intervention works, and why it works in the Vietnamese context and also of academics' reflection and reflective practice.

The study includes recommendations for the use of TT-SET augmented with POT for promoting teacher reflection that may lead to changes in practice by addressing necessary conditions for the intervention to be effective. Further research is recommended into the nature of the peer relationship and the characteristics of the peer for POT (e.g., in terms of age, experience, gender, and discipline), the impact of
\end{abstract}


contextual factors, the role of leaders in creating the culture of the learning community, the timing of the intervention, and the use of students' learning outcomes to evaluate the effectiveness of changes. 


\section{Acknowledgments}

I am indebted to a number of people for their support over the completion of this thesis. I am indebted to my supervisors, Associate Professor Liz Jones and Dr. Dayle Anderson, who offered expertise support and supervised the development of the thesis. Their support has facilitated my professional growth as a researcher. The two supervisors jointly offered me their guidance and thorough critique with their expertise. I also owe them gratitude for their patience and encouragement throughout the process of the study.

Having an opportunity for completing my thesis in New Zealand, I would like to send my gratitude to the Vietnamese Ministry of Education and Training and Victoria University of Wellington for awarding me the scholarship. My sincere appreciation also goes to the Vietnam International Education Development and Victoria University Scholarship Committee for providing me with the required funding for my study.

I would like to acknowledge the 11 academics who kindly agreed to participate in this study and share their experiences. The data collection would not have been completed without their support. I give my sincere thanks to these participants for their willingness, reflectively sharing their experience, and arranging time in their heavy schedules to participate in this study. Without their help, the insights into the implementation of the theoretical model would not have been achieved.

My gratitude is due to the postgraduate administrative office personnel for providing the necessities during my $\mathrm{PhD}$ study period. I highly appreciate their care and service.

I sincerely thank my employer, the university for which I have been working, for providing me with a chance to pursue further study.

I owe thanks to Dr. Judith Loveridge for her tremendous help and advice that supported me in refining the thesis. I am grateful to Dr. Louise Starkey and Professor Emerita Luanna Meyer, who offered me useful recommendations. My thanks are due to academics and friends at the Faculty of Education, Victoria University of Wellington who have offered support in their own different ways, including Dr. Linda Hogg, who organised cohort meetings. I offer my sincere thanks to other friends for their endless encouragement that inspired me towards the completion of this thesis. 
Special thanks are due to all my family members for their support, love, and encouragement that promoted my strength to study far away from home. They have always been guarding my academic efforts and believed in my ability to succeed. I would like to dedicate this $\mathrm{PhD}$ thesis to my grandfather and my mother who have sacrificed their entire life to me. My thanks are given to my beloved wife, Pham Thi Huong, and my sons, Pham Gia Bao and Nguyen Quang Kiet for their love and support throughout the years. 


\section{Abbreviations}

DEB Debriefing

EFL English as a foreign language

ESP English for Specific Purposes

INT Interview

POF Peer observer's feedback form

POT Peer observation of teaching

RJ Researcher journal

TRR Teacher's reflection report form

TT-SET Teacher-tailored student evaluation of teaching

SEEQ Students' Evaluation of Educational Quality

S-SET Standardised student evaluation of teaching

VID Video 


\section{Table of Contents}

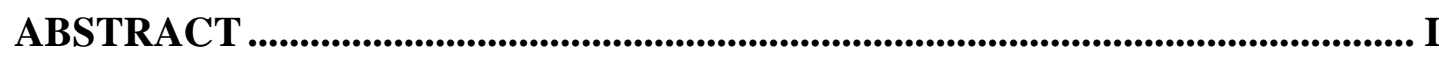

ACKNOWLEDGMENTS ............................................................................................ III

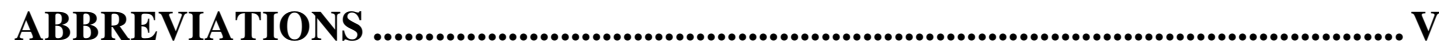

TABLE OF CONTENTS ............................................................................

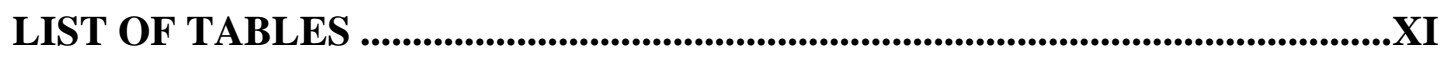

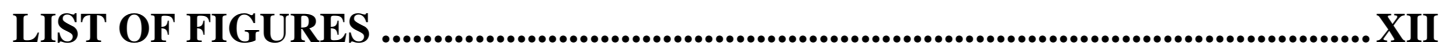

CHAPTER 1. BACKGROUND OF THE STUDY .............................................. 1

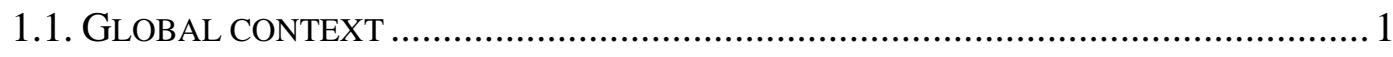

1.2. TERTIARY EDUCATION IN VIETNAM......................................................... 3

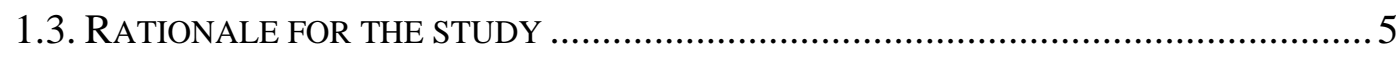

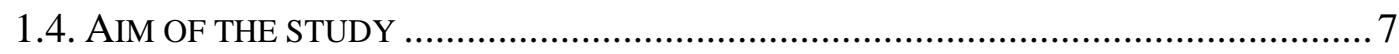

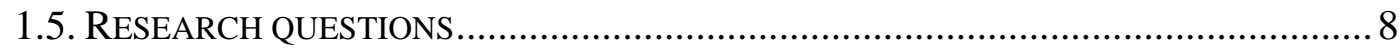

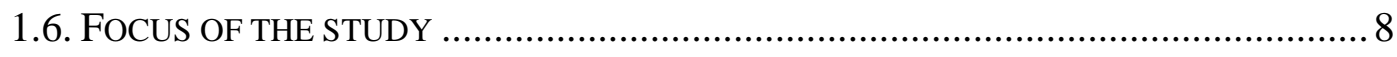

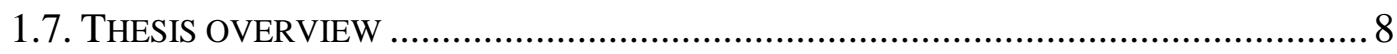

CHAPTER 2. LITERATURE REVIEW ................................................... 11

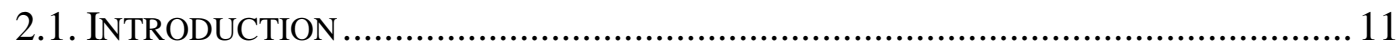

2.2. TEACHERS' PEDAGOGICAL REASONING, KNOWLEDGE, AND BELIEFS................. 12

2.2.1. Teachers' pedagogical reasoning ......................................................... 12

2.2.2. Teachers ' professional knowledge and beliefs....................................... 14

2.2.2.1. How teachers' knowledge can be developed............................................... 14

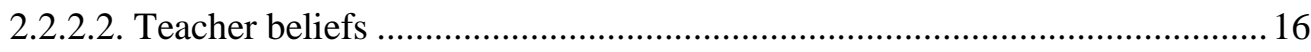

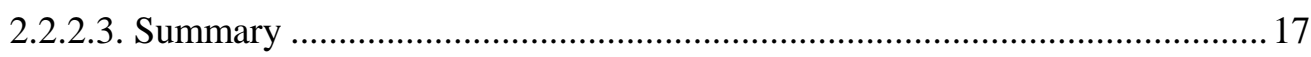

2.3. SELF-EFFICACY AND AUTONOMY: MOTIVATION FOR ACTIONS ......................... 18

2.3.1. Teacher self-efficacy .......................................................................... 18

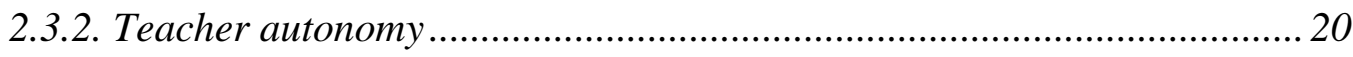

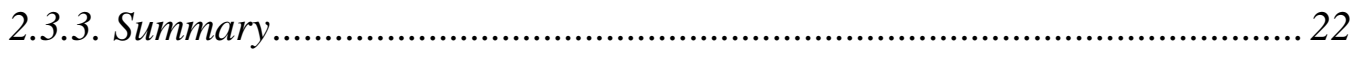

2.4. TEACHER REFLECTION AND REFLECTIVE PRACTICE ...................................... 22

2.4.1. Reflection contributing to professional learning ................................... 22

2.4.1.1. Conceptualising reflection and reflective practice .....................................22

2.4.1.2. How reflection may contribute to learning ...................................................2 29

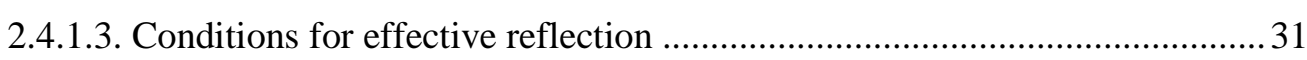

2.4.2. Academics' critically reflective lenses ................................................... 32

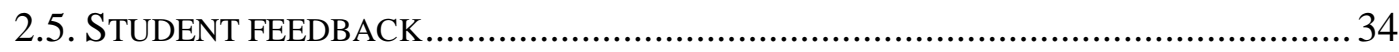


2.5.1. Purposes of student feedback in tertiary education ................................. 35

2.5.2. Formative SETs triggering reflection .................................................. 36

2.5.3. Conditions for effective SETs and collecting TT-SET........................... 37

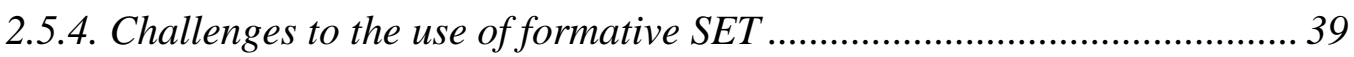

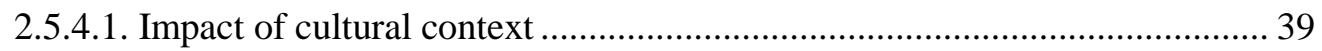

2.5.4.2. Academics' perception of student feedback .............................................. 40

2.5.5. SET as a complementary source used with consultation .......................... 42

2.6. PEER OBSERVATION OF TEACHING (POT) ...................................................... 43

2.6.1. Definition and purposes of POT ............................................................ 44

2.6.2. Structured POT procedure for reflection .................................................. 46

2.6.3. Effectiveness of formative POT ............................................................. 48

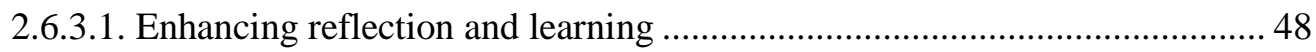

2.6.3.2. Increasing perceived changes in current practice and aspirations for future

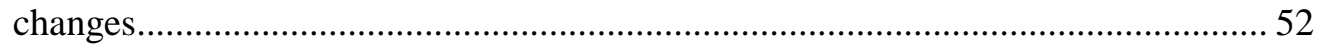

2.6.3.3. Enhancing confidence and self-efficacy .................................................. 53

2.6.3.4. Enhancing collaboration and collegiality ..................................................... 55

2.6.4. Conditions for POT to be effective ........................................................... 56

2.6.5. Challenges to peer observation of teaching.............................................. 58

2.6.6. Cultural context of implementation ........................................................... 61

2.6.6.1. Overview of cultural variations in relationships ....................................... 61

2.6.6.2. Vietnamese cultural context concerning relationships.................................. 62

2.7. SUMMARY OF THE LITERATURE REVIEW AND RESEARCH GAP ............................63

2.7.1. Summary of findings from the literature on formative SET and POT .... 63

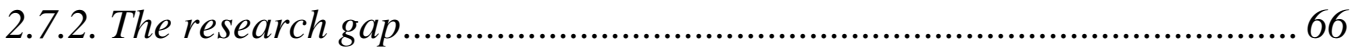

CHAPTER 3. METHODOLOGY ................................................................69

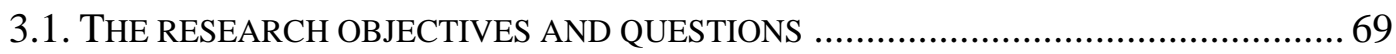

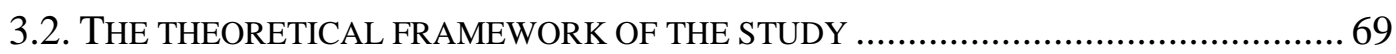

3.3. RESEARCH PARADIGM, METHODOLOGICAL APPROACH, AND METHOD ............... 77

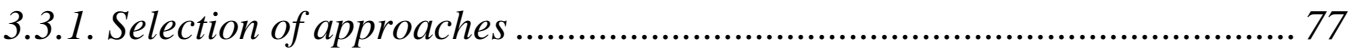

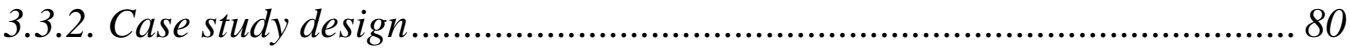

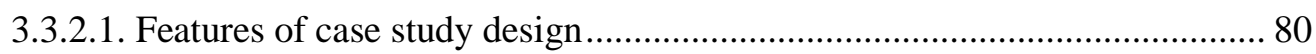

3.3.2.2. Relevance of case study design for the research ........................................ 81

3.3.2.3. Necessity of propositions for the research ................................................ 82

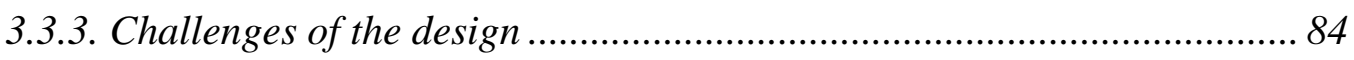

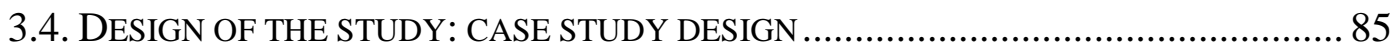

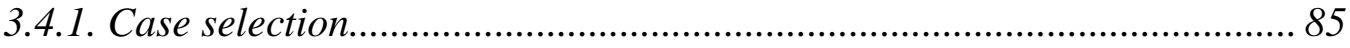

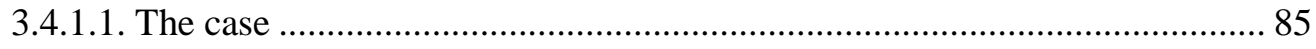

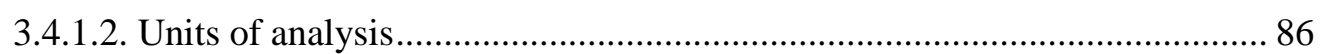




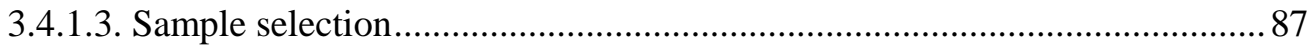

3.4.2. Propositions in accordance with the research questions........................ 88

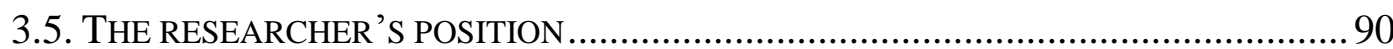

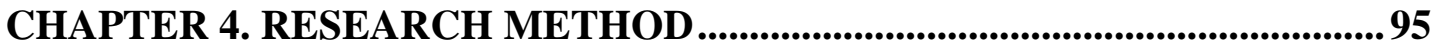

4.1. INSTITUTIONAL AND PROGRAMME CONTEXT ............................................... 95

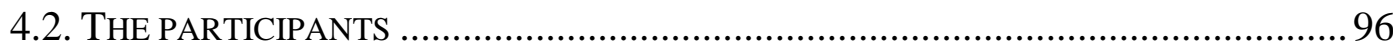

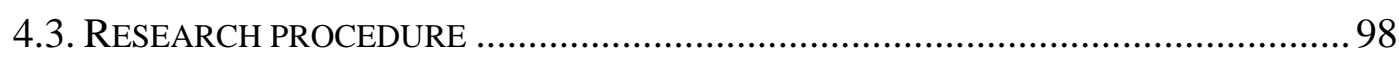

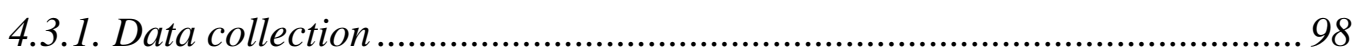

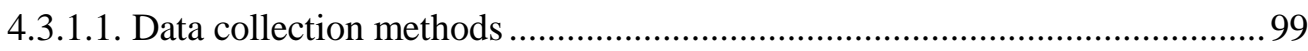

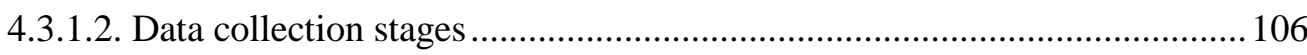

4.3.2. Relationship of data to research questions and propositions ............... 109

4.3.3. Data analysis........................................................................................... 109

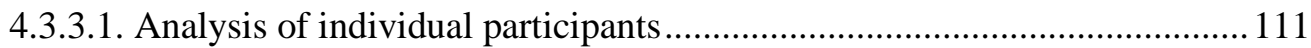

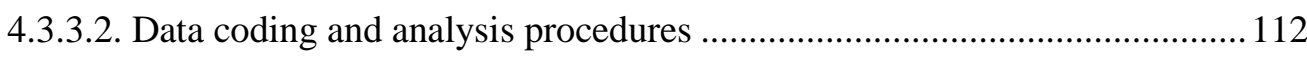

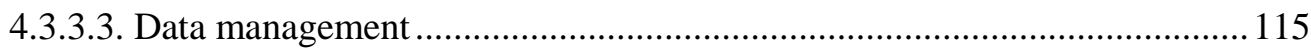

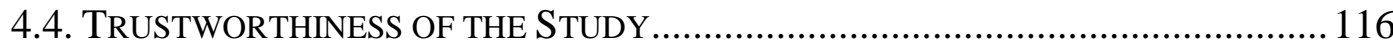

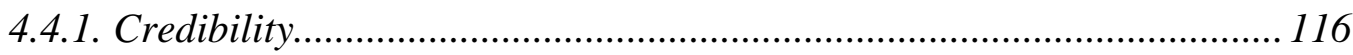

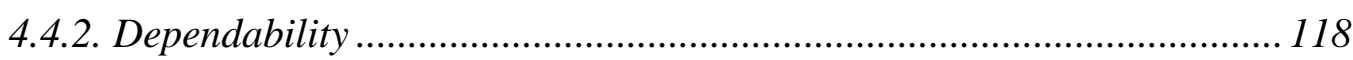

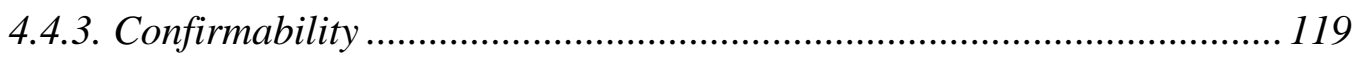

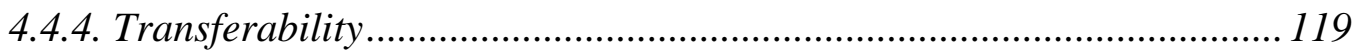

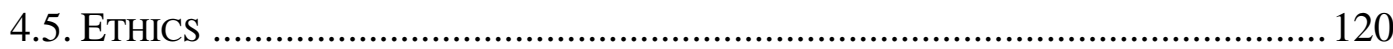

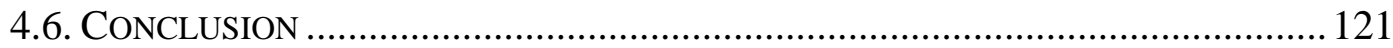

CHAPTER 5. ACADEMICS' EXPERIENCES OF TT-SET AUGMENTED

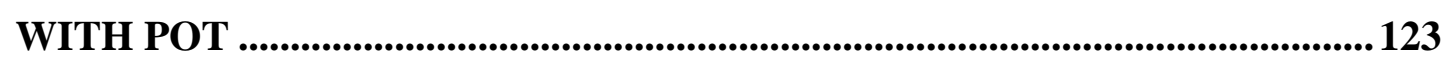

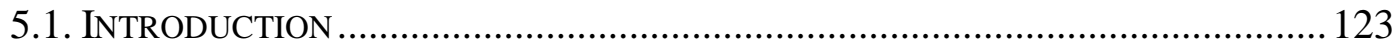

5.2. PERCEPTIONS AND PERCEIVED IMPACT …................................................ 125

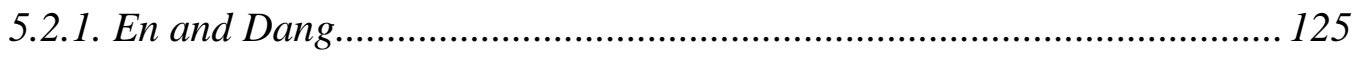

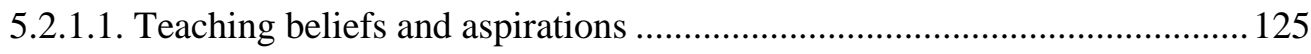

5.2.1.2. Perceived values: always highly beneficial ............................................... 127

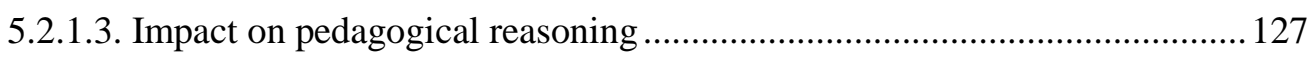

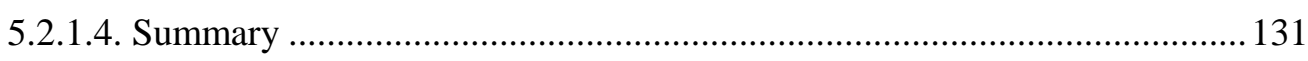

5.2.2. Dieu, Hang, and Khoa ........................................................................ 132

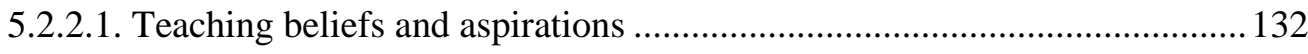

5.2.2.2. Perceived values: mostly beneficial .......................................................... 135

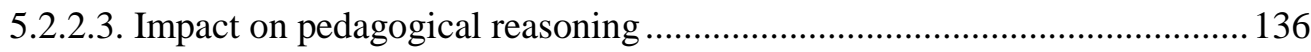

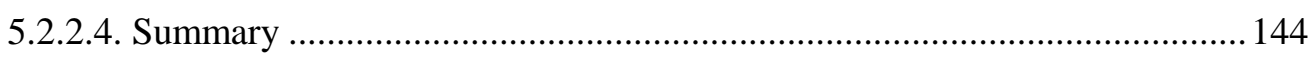

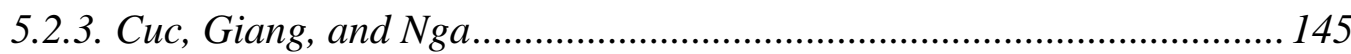




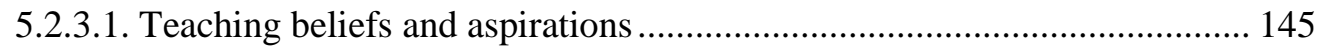

5.2.3.2. Perceived values: some beneficial aspects ............................................. 148

5.2.3.3. Impact on pedagogical reasoning........................................................... 149

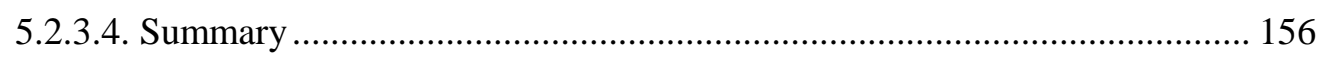

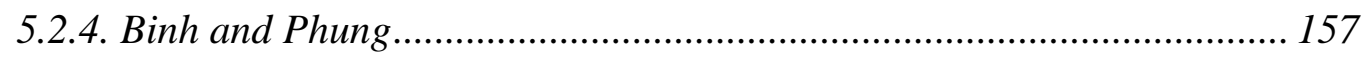

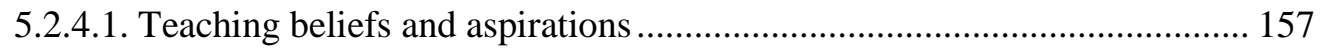

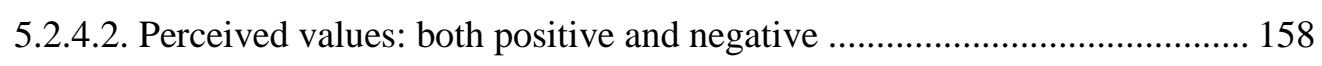

5.2.4.3. Impact on pedagogical reasoning........................................................... 159

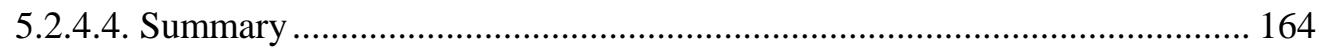

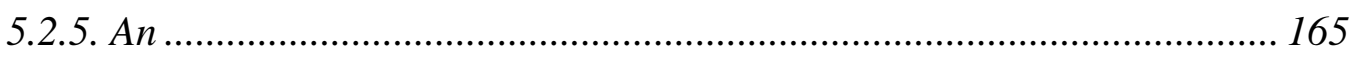

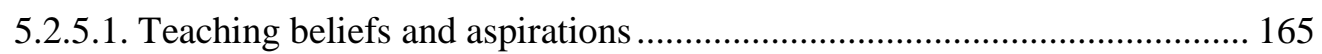

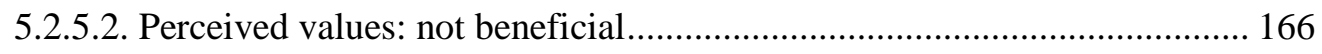

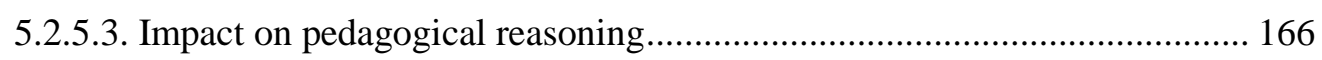

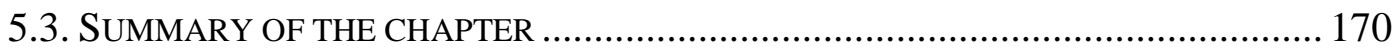

CHAPTER 6. ADDRESSING THE RESEARCH QUESTIONS..................... 171

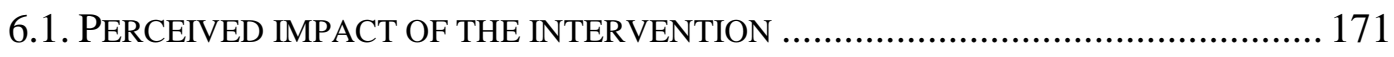

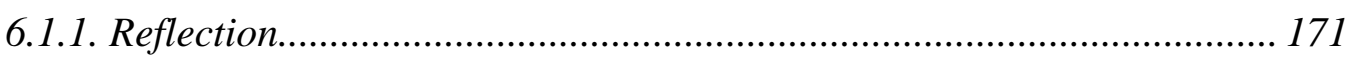

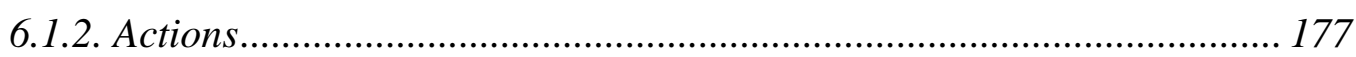

6.1.3. Attitudes: increased confidence and self-efficacy ............................... 180

6.1.4. Enhanced collegiality and collaboration .............................................. 182

6.2. ACADEMICS' PERCEPTIONS OF THE INTERVENTION....................................... 184

6.2.1. TT-SET as a possible promoter of reflection ....................................... 185

6.2.2. POT as possible promoter of reflection ................................................. 190

6.2.3. TT-SET plus POT: promoting reflection through structured steps........ 192

6.2.4. Improving the intervention to encourage participation ........................ 196

6.3. CHALLENGES TO THE IMPLEMENTATION........................................................... 198

6.3.1. Perception of TT-SET as a challenge ................................................. 198

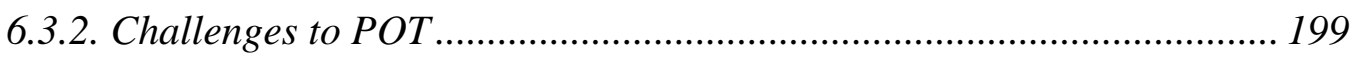

6.3.2.1. Perception of potential challenges ............................................................... 199

6.3.2.2. Actual experienced challenges................................................................. 202

6.3.2.3. Comparing potential and actual perceived challenges .............................. 206

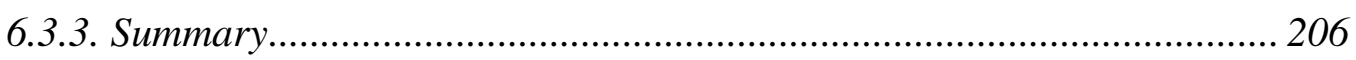

6.4. CHANGES IN ACADEMICS’ PEDAGOGICAL REASONING ................................... 207

6.4.1. Enhanced reflection and new comprehensions ................................... 207

6.4.2. Perceived changes and future plans .................................................. 208

6.4.3. Enhanced confidence, self-efficacy and autonomy .............................. 209

6.4.4. Enhanced collaboration and collegiality ............................................ 209

6.4.5. Actual challenges and possible solutions.............................................. 210 
CHAPTER 7. DISCUSSION OF FINDINGS ................................................ 213

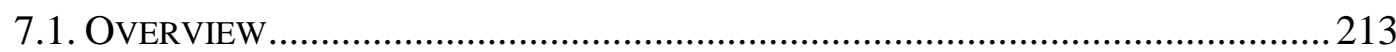

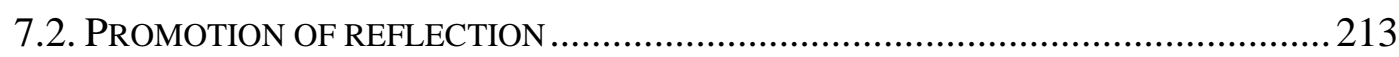

7.2.1. Features that promote reflection ........................................................ 213

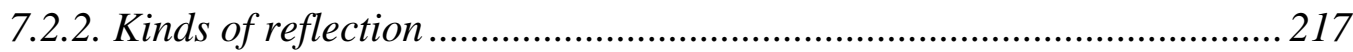

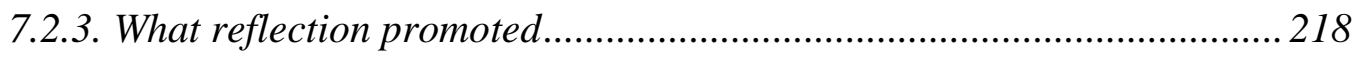

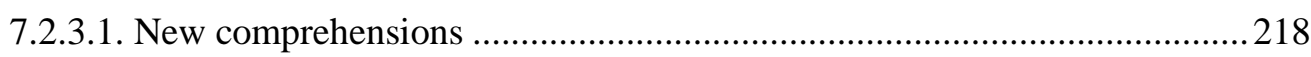

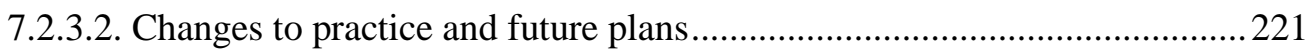

7.2.3.3. Enhanced confidence, self-efficacy and autonomy .....................................225

7.2.3.4. Nurtured collaboration and collegiality through POT .................................. 230

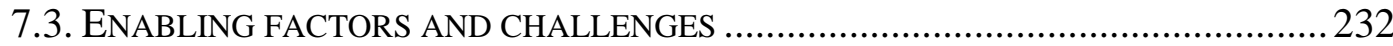

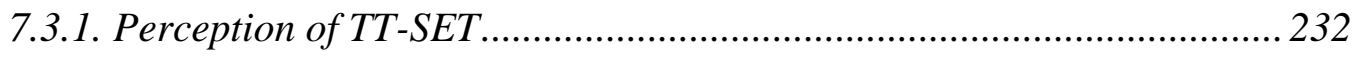

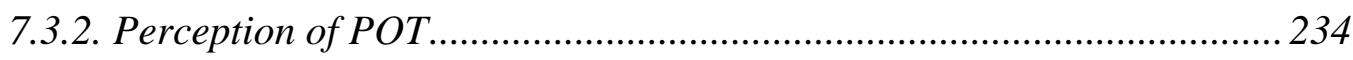

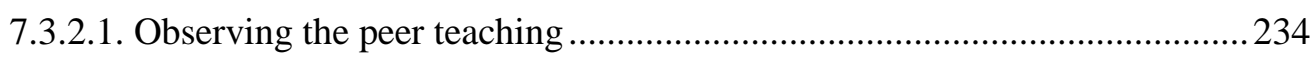

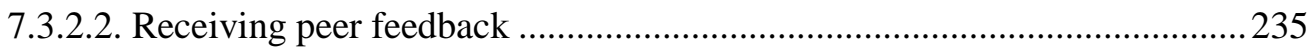

7.3.3. Time resource for POT ....................................................................... 240

7.4. THE USEFULNESS OF THE THEORETICAL FRAMEWORK ................................... 243

CHAPTER 8. CONCLUDING REMARKS ........................................................... 247

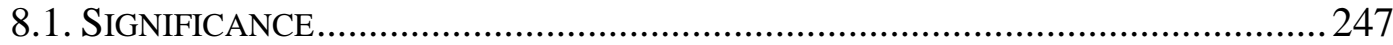

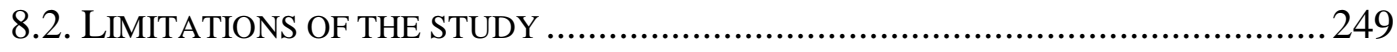

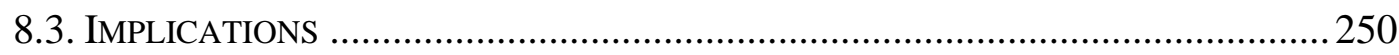

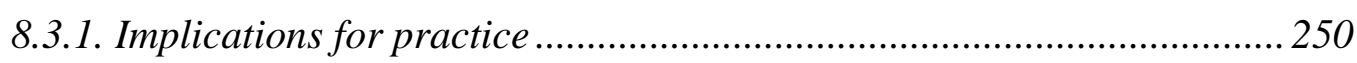

8.3.2. Implications for institutional culture and policies ............................... 252

8.3.2.1. When the benefits outweigh the cost..........................................................253

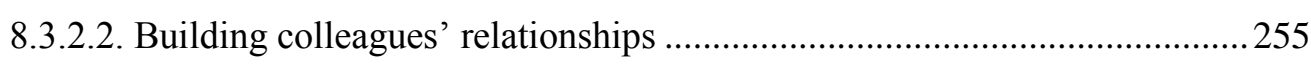

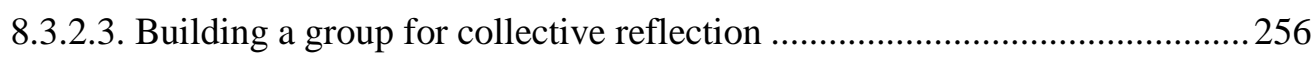

8.4. RECOMMENDATIONS FOR FURTHER RESEARCH........................................... 257

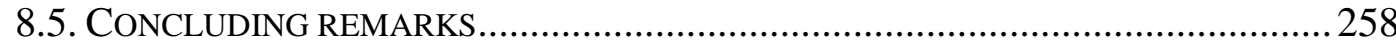

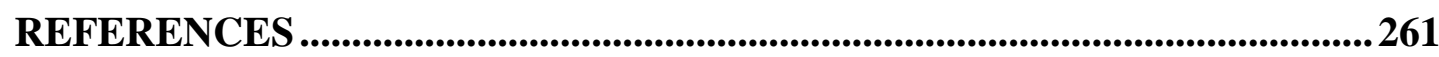

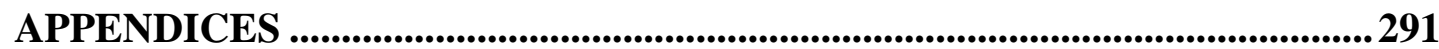




\section{List of Tables}

Table 4.1. The peers for peer observation of teaching ........................................... 97

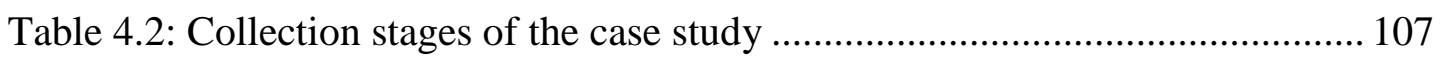

Table 4.3: The sources that generate data in relation to the research questions and the

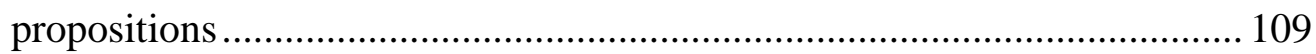

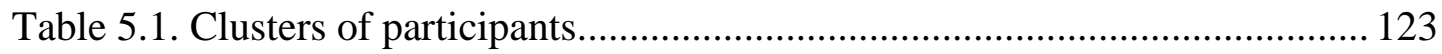

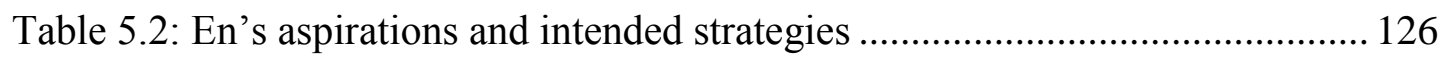

Table 5.3: Dang's aspirations and intended strategies ........................................... 126

Table 5.4: Dieu's aspirations and intended strategies ............................................ 133

Table 5.5: Hang's aspirations and intended strategies .......................................... 134

Table 5.6: Khoa's aspirations and intended strategies .......................................... 135

Table 5.7: Cuc's aspirations and intended strategies ............................................ 146

Table 5.8: Giang's aspirations and intended strategies ......................................... 147

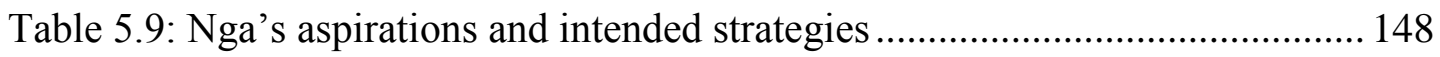

Table 5.10: Binh's aspirations and intended strategies .......................................... 157

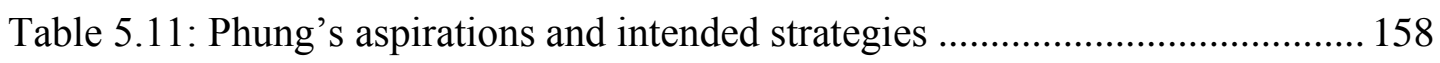

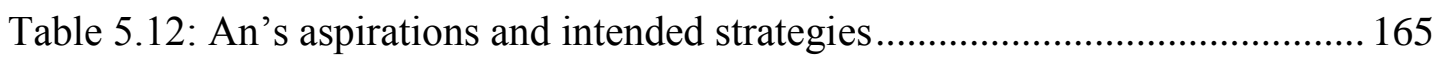

Table 6.1: Academics' enhanced reflection and other outcomes........................... 173

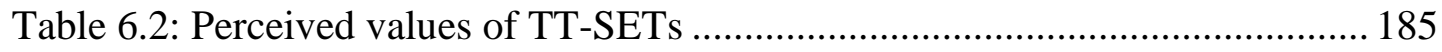

Table 6.3: The role of the TT-SET in academics' self-directed learning ................ 188

Table 6.4: Academics' expectation of end-of-term TT-SET results ........................ 189

Table 6.5: Perceived values of peer feedback and classroom observations.............. 191

Table 6.6: Usefulness of steps in the current intervention for reflection and learning 194

Table 6.7: Potential perceived challenges to POT …............................................... 200 


\section{List of Figures}

Figure 3.1: The theoretical framework for the study (developed from Shulman's (1987) pedagogical reasoning and action and LaBoskey's (1993) reflection model) .73

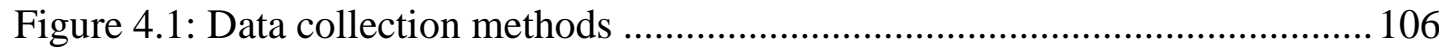

Figure 4.2: Data Analysis in Qualitative Research adapted from Creswell (2009) . 113

Figure 5.1: Summary logic model for each academic............................................. 124

Figure 5.2: Summary logic model for En............................................................ 129

Figure 5.3: Summary logic model for Dang .................................................... 131

Figure 5.4: Summary logic model for Dieu ...................................................... 138

Figure 5.5: Summary logic model for Hang ..................................................... 141

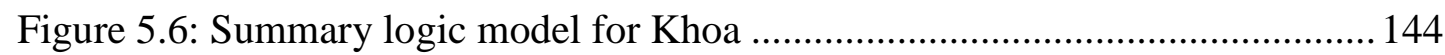

Figure 5.7: Summary logic model for Cuc ......................................................... 151

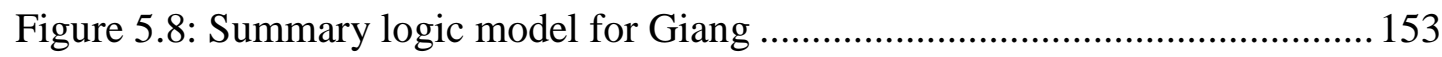

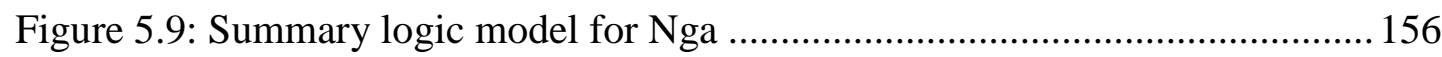

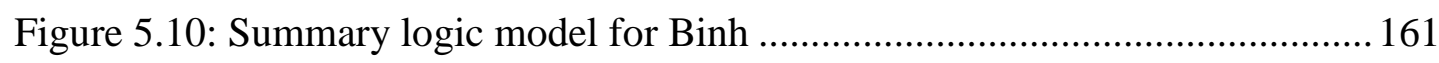

Figure 5.11: Summary logic model for Phung .................................................... 164

Figure 5.12: Summary logic model for An ...................................................... 169

Figure 7.1: Perceived enabling factors and challenges to the intervention.............. 242

Figure 7.2: Proposed model for the implementation of TT-SET and POT .............. 245 


\section{Chapter 1. Background of the Study}

\subsection{Global context}

Tertiary education has an enormous impact on individuals and society. As McArthur (2011) argued, tertiary education "has a social, an economic and an educative role that extends well beyond its walls and its own students" (p. 746). By this, she means that tertiary education not only develops students' identities with critical and political sensibilities but also contributes to the social, cultural, economic and political development of the society. There have been contested visions around the role and purpose of tertiary education, particularly in the marketization of knowledge production in a global economy (Donnelly, 2007). Its economic purpose is sometimes used in the narrowest sense: employability. Tertiary education is believed to be responsible for improving employment skills to stay competitive in the knowledgedriven global economy (Hallinger, 2010; Hénard, 2010). Regardless of whether the purpose of tertiary education is in its narrow or wider sense (e.g., the development of the individual as a model citizen), the need for good teaching is the same. The demand requires attention to be paid to the quality of tertiary teaching. Academics contribute greatly to the quality of education because they possess a particular body of knowledge and specialised capabilities that affect students, who are the subjects of the educational process. Thus, with this decisive role, academics' teaching skills or pedagogy can impact the quality of teaching and students' learning outcomes. Great effort is needed to build long-term capacity for improvement through processes such as the sustained development of teachers' skills (Hargreaves \& Fink, 2003). It is therefore necessary to promote models to enhance academics' learning about teaching. Many researchers like Darling-Hammond (2006) argue that engaging academics in professional sharing and critical reflection and assisting them to adapt knowledge to specific contexts can boost this process of learning about teaching.

To enhance economic development most developing nations such as Vietnam have promoted mass tertiary education to provide their economies with a highly skilled workforce. The increasing number of students and the expansion of private and public colleges and universities in these countries have brought about concern. In the report on "higher education in developing countries", The World Bank (2000) stated: 
Expansion, both public and private, has been unbridled, unplanned, and often chaotic. The results - deterioration in average quality, continuing inter-regional, inter-country, and intra-country inequities, and increased for-profit provision of higher education - could all have serious consequences. (p. 27)

The World Bank's report also addressed the lack of well-qualified and highly motivated academics who are the key to the quality of tertiary education institutions. It identified several problems such as academics' limited graduate training or outmoded teaching methods that result in rote learning. Low pay for academics and ill-conceived incentive structures in many developing countries make it difficult to improve the quality of academics (The World Bank, 2000). The World Bank also pointed out another reason for the low quality of teaching at tertiary level in these countries: "Many faculty work part-time at several institutions, devote little attention to research or to improving their teaching, and play little or no role in the life of the institutions employing them" (p. 24). Among other reasons, the lack of sound academic training and professional development also results in the poor quality of teaching. The case of academics who teach English as a foreign language is no exception. There is a rising need for academic professional development, particularly for teachers of English at tertiary level.

The quality of English language teaching plays an important role in development, particularly in developing countries. English, the predominant language for development in these countries (Shamim, 2011), has played several roles, as Coleman (2010) identified, including:

increasing individuals' employability; enabling international collaboration and co-operation; providing access to research and information; facilitating the international mobility of students, tourists, workers and others; facilitating disaster relief and disaster preparedness; and acting as an impartial language in contexts of disharmony. (Coleman, 2010, p. 15).

One example of the role of English in employability is related to the presence of many multinational companies in developing countries. This demands employees, whose mother tongue is not English, to have a sufficient command of English for communication.

Concern for the quality of English language teaching and its essential role in developing countries requires adequate forms of training or professional development 
to enhance academics' teaching quality. In a study (Nunan, 2003) on the impact of English as a global language on educational policies and practices in the Asia-Pacific region including developing countries like Vietnam, the issue of inadequately trained and skilled English language teachers is among other significant problems that have been revealed. The poor quality of English language teaching in these Asia-Pacific countries is believed to result from a lack of good academic training and professional development. Thus, reforms need to be initiated in English language teacher education at both the pre-service and in-service levels.

\subsection{Tertiary education in Vietnam}

The need for measures to improve the quality of tertiary teaching is also impacted by the Vietnamese tertiary educational context. The significance of higher education development, educational assessment, and quality assurance is a focus in the Vietnamese Government's Resolution 14/2005/NQ-CP on 'Substantial and Comprehensive Reforms of Vietnam's Tertiary Education in the 2006-2020 period' promulgated by the Prime Minister in November, 2005. One of the general objectives of this innovation project is to develop well-qualified tertiary teaching and administrative staff with professional ethics, expertise, and advanced pedagogy and administration (The Vietnamese Government, 2005).

Vietnam, willing to establish mutual and close ties with other countries, has conducted an open-door policy toward its integration into the world. Vietnam has integrated into the rest of the world as a World Trade Organisation member since 2007 after its membership of ASEAN (The Association of Southeast Asian Nations) and participation in AFTA (ASEAN Free Trade Area) in 1995. English has become widely used for international communication to facilitate Vietnam's trade, business, educational, and political relations with other countries. Thus, the Vietnamese government has put emphasis on the importance of English as part of Vietnam's socioeconomic development. Therefore, there is a goal to improve the quality of English teaching at the tertiary level. Communication with the rest of the world requires Vietnamese to have a good command of English, so the importance of English has increased over time. Thus, English has become the most popular foreign language taught and learnt at all levels of education in Vietnam, particularly in Vietnamese tertiary education institutions. 
In Vietnam, the quality of English language teaching has long been a concern. One of the problems is inadequately trained and skilled teachers. Therefore, improving the quality of foreign language teaching is one of the government's several measures to reform tertiary education along with the establishment of a quality assurance system and encouraging connection between teaching and research. Major reform of foreign language education was emphasised in the Prime Minister's decision 1400/QĐ-TTg on teaching and learning foreign languages in the national education system from 2008 to 2020 project. The objective of this project is to implement radical changes that enable Vietnamese graduates to use foreign languages properly in their multicultural work and study environment (The Prime Minister, 2008). As part of this initiative, English as a foreign language (EFL) or English for Specific Purposes (ESP) has become a mandatory subject in tertiary education.

Tertiary education institutions in Vietnam have taken several measures to improve teaching. However, important professional development activities such as coaching, supervision, and mentoring are under-utilised compared with other most-preferred forms of learning such as formal degree programmes and self-study (Hoa, 2008). For example, it is possible to locate just a few studies into peer observation of teaching such as critical friend groups for beginner teachers (Long \& Hoa, 2010) and peer mentoring of beginning EFL academics (Hoa, 2008) or pre-service EFL teachers (Hoa, 2013) at tertiary level in Vietnam. This indicates that academic professional development through peer observation of teaching, seminars, workshops, or informal teaching dialogue receives little attention (Hiep, 2001).

Teaching Business English, as a particular form of teaching ESP which is commonly implemented for students majoring in economics or business related areas, is seen as problematic in the current context of English language teaching in Vietnam. In a study involving Business English academics at three universities in Vietnam, Huong (2014) found that the quality of Business English teaching appeared to be affected by factors such as designing business English programmes, problematic staffing procedures, and academics' limited research activities, low pay and ineffective 'emulating' schemes (where academics make efforts to improve achievement in annual appraisal events), and academics' part-time jobs or expanded roles. These academics reported that they had limited time to engage in teaching improvement because of working part time. Many believed that for teaching to be improved, academics needed to have appropriate 
professional and pedagogical knowledge to enable student learning, engage in continuous professional development, and gain understanding of student needs, together with commitment and responsibility. Working part time may add to academics' work burden; yet, collaboration between academics can help ease their workload and reinvigorate their teaching (Eddy \& Garza Mitchell, 2012). One of the measures for improving teaching quality can be the implementation of professional development models that trigger academics' reflection and reflective practice and their commitment to learning about teaching. As Tran et al. (2014) suggested, for Vietnam's tertiary education to be improved, priority should be given to "improved resourcing over time, better staff development and reform in educational governance and in government itself" (p. 236).

\subsection{Rationale for the study}

My interest in pursuing ways to help academics reflect and learn about teaching derives from the pressing need to improve teaching in tertiary education, particularly the quality of teaching ESP. From my personal experience as a lecturer of English, if academics reflect on practice and gain deeper understanding of practice, their teaching capacity may develop. Students and peer colleagues are those who are in direct contact with academics' performance and thus appear to be a useful information source about teaching. Years ago, I often collected student feedback on my teaching with my own student evaluation of teaching (SET) form which focused on the aspects of teaching I wanted to know about. I selected items I needed from a standardised SET, making the teacher-tailored SET (TT-SET). Then, I made changes to my practice. However, I thought seeing practice through the student lens was not sufficient, so I looked for a professional viewpoint. Eventually, I joined in reciprocal peer observation of teaching (POT) with other colleagues in my department. I learnt new teaching techniques and strategies, some of which appeared 'odd' to me but could engage students in learning. I felt comfortable receiving feedback from my peers because it was not evaluative and did not threaten my reputation. Both forms of feedback have their own weaknesses with respect to their use as a trigger for reflection and reflective practice, and I believed that both could supplement each other in informing teaching.

Formative SET and POT for teaching improvement are among the models of professional development employed by tertiary education institutions worldwide. SET 
has been commonly used (Yao \& Grady, 2005) and POT has become a popular practice in tertiary education (Bennett \& Barp, 2008). A number of studies have demonstrated positive results as SET helped academics to improve their practice such as Aultman (2006), Cohen (1980), Ramsden (1991), and Yao and Grady (2005). To make effective use of SETs and provide a professional perspective on practice for teaching improvement, it is suggested that SETs be augmented with forms of consultation (e.g., Dresel \& Rindermann, 2011; Lang \& Kersting, 2007; Penny \& Coe, 2004; Rindermann, Kohler, \& Meisenberg, 2007). One of these forms can be through critical friendships (Wichadee, 2012). POT, which has been viewed as promoting reflection and learning (e.g., Bell \& Cooper, 2011; Sullivan, Buckle, Nicky, \& Atkinson, 2012) when used for formative purposes, may be used to supplement SET because peer colleagues as insiders in the profession can provide a professional viewpoint on teaching.

It can be drawn from the global context that formative SET and POT are relevant processes for engaging academics in reflection and professional learning, which may lead to their enhanced practice. Yet, SET and POT may not be automatically effective for improving teaching and successfully implemented in all contexts because of the possible impacts of the specific organisational or cultural context (Barnard et al., 2015). Therefore, there is a need to explore how they work in the Vietnamese context. In Vietnamese tertiary education, the use of SETs and POT for teaching improvement have relevance. Students have been encouraged to contribute to the teaching-learning process, and SETs have been increasingly used (Dung \& Mcinnis, 2002). Despite concerns about the SET shortcomings (e.g., academics' vulnerability to criticism and threat to their high traditional status), the researchers suggested that academics can gain valid information from SET and use it to improve performance. In addition to formative SETs, POT may be valuable for academics' reflection and reflective practice. However, little attention is paid to POT as a formal learning activity for academics in Vietnamese tertiary education (i.e., it is rarely used and mostly is selfinitiated) for fear of threats to academics' privileged position and the need for greater resources such as time and energy. Although POT is not widely used in Vietnam (Hiep, 2001), some recent studies (e.g., Long and Hoa, 2010; Hoa, 2008, 2013) that employed POT as a strategy have revealed the effective use of colleagues as a reflective lens for pre-service or beginner EFL academics to improve teaching. Despite the positive 
outcomes, I suspect that POT may face cultural challenges related to interpersonal interactions because harmony is of primary concern in collectivist cultures (Mai, Terlouw, \& Pilot, 2005). The overview of the use of SETs and POT indicates that few studies have employed TT-SET augmented with POT, so I am motivated to explore how the intervention works for Vietnamese academics.

The Vietnamese context may determine the extent to which formative TT-SET augmented with POT is successful as a process for promoting reflection and learning about teaching. Although I may predict possible challenging cultural factors for the implementation of the intervention in Vietnam, I will keep the model of the intervention as it is originally intended in Western research. Research is contextually situated, and I aim to examine the extent to which Vietnamese cultural factors may impact its implementation. Therefore, I want to explore what works, what does not work with the intervention, and how to make it work better in the future within the Vietnamese context.

\subsection{Aim of the study}

This study is a case examination of TT-SET augmented with POT in a Vietnamese university. It explores how the intervention (TT-SET augmented with face-to-face collaborative POT) impacts on academics' pedagogical reasoning, particularly their reflection and changes they make with an objective to improve teaching, by:

i. documenting academics' reflection through the process of pedagogical reasoning based on feedback from students and peers;

ii. examining the perceived impact of the intervention on academics' practice, beliefs and attitudes;

iii. recording their perceptions of the effectiveness of TT-SET augmented with POT for promoting their reflection and reflective practice in the pedagogical reasoning process;

iv. identifying possible challenges to the implementation of TT-SET augmented with POT in the Vietnamese context. 


\subsection{Research questions}

In order to achieve the objectives, the following research questions are formulated. There is one main question with three sub-questions:

In the context of a Vietnamese university, how does TT-SET augmented with POT impact on academics' pedagogical reasoning?

There are three sub-questions:

1. What is the perceived impact of TT-SET augmented with POT on academics' practice, beliefs, and attitudes?

2. What are academics' perceptions of the effectiveness of TT-SET augmented with POT for promoting their reflection in the process of pedagogical reasoning?

3. What are possible challenges to the implementation of TT-SET augmented with POT in Vietnam's context?

\subsection{Focus of the study}

This research locates the area for the implementation of TT-SET augmented with POT for formative purposes in tertiary education. Through the literature review on the use of SET and POT, it is noted that the interventions are used worldwide, focusing on their effectiveness for academics' professional learning and development. There have been few studies involving POT in Vietnam. These were conducted with pre-service or beginner EFL teachers and did not discuss the impact of cultural factors on the implementation of these intervention processes. This study examines how TT-SET augmented with POT impacts more experienced academics' pedagogical reasoning, with a focus on their reflection, learning and changes to practice and discusses the perceived impacts of cultural factors on its implementation.

\subsection{Thesis overview}

This chapter has described the context for this study and developed the research questions that guide it. Chapter 2 establishes the argument for the theoretical framework which is presented in Chapter 3 through the review of the literature on: teachers' pedagogical reasoning, beliefs, and knowledge; self-efficacy and autonomy; reflection and reflective practice; and the students' and colleagues' lenses for reflection with SET and POT. The research gap is then identified through the summary 
of findings from the review of literature. Chapter 3 presents the research objectives and questions, and the theoretical framework of the study which is developed based on the literature review, and the methodology. Chapter 4 presents the research context, the participants, and the research methods including data collection and analysis used for this study. Chapter 5 provides an overall picture of participants' experience of TTSET augmented with POT. Chapter 6 examines the data and findings across participants and answers the research questions. Chapter 7 discusses the findings of the study in relation to the literature. Chapter 8 presents the study's significance and limitations, discusses implications, proposes recommendations for future research, and ends with concluding remarks. 



\section{Chapter 2. Literature Review}

\subsection{Introduction}

Learning about teaching may result from reflection which can be triggered by internal factors such as self-assessment and external factors such as feedback from students and colleagues. LaBoskey's (1993) reflective model suggests that reflection may lead to new comprehension, which may result in actions to solve practical problems. The learning may involve the adoption of new action strategies (single-loop learning) or the changing of one's assumptions (double-loop learning) (Argyris \& Schön, 1974). Reflection may need structural aids such as student feedback and colleague's consultation to be effective. With sufficient resources or prerequisites for reflection, learning can occur and may lead to actions to enhance practice.

How teachers decide on what resources and teaching methods to use in their classroom can be explained in Shulman's idea of teaching that emphasises comprehension and reasoning, transformation and reflection. Shulman's (1987) model of pedagogical reasoning and action was developed as a foundation for teaching reform. The model identifies actions that teachers take during their practice; one of these actions is reflection. As it portrays reflective practice during the teaching process, the model is relevant for examining academics' reflection and reflective practice, knowledge development, and actions to improve practice in the process of pedagogical reasoning. Academics' desire to act as reflective professionals may stem from some source of motivation. When academics feel that they are competent (a sense of self-efficacy) and empowered to take control over aspects related to their practice (a sense of autonomy), they may take actions to change. Self-efficacy and autonomy contribute to intrinsic motivation (Ryan \& Deci, 2000a), which is seen as more powerful than extrinsic motivators in triggering adults' learning (Knowles, Holton, \& Swanson, 2005). It seems useful to find a mechanism to enhance academics' reflection as well as selfefficacy and autonomy to facilitate teaching improvement.

Multiple sources of information may be necessary for academics to grasp a complete picture of their practice (Algozzine et al., 2004). For effective reflection, academics may need to seek lenses. Brookfield (1995) proposes the four critically reflective lenses: teachers' autobiographies as learners and teachers, students' eyes, colleagues' 
experiences, and theoretical literature. They appear to be appropriate sources for reflection because, for effective reflection, teachers need to examine practice from different angles (Loughran, 2002). Yet, this study focuses on student and colleagues for their external contextual information on practice (noting that theoretical literature is an external lens as well). Students are direct recipients of the teaching process; therefore, their opinion about teaching practice is important for academics' reflection. Meanwhile, colleagues can provide a professional perspective on teaching.

This literature review aims to explore academics' reflection and reflective practice and learning about teaching in tertiary education. The review begins by examining the literature concerning teachers' pedagogical reasoning, beliefs (including self-efficacy and autonomy), and knowledge. This is followed with exploration of literature on models of reflection and learning theories, focusing on how reflection and reflective practice contribute to professional learning. Then, the review looks at two lenses for reflection: students and colleagues. It discusses the formative purpose of the lenses in the forms of SET and POT. Their effectiveness and challenges with reference to the context of implementation are also considered to see whether SET and POT can be effectively used for triggering academics' reflection and learning. Finally, the findings from the literature are summarised, which signals the research gap and the research question to guide the study.

\subsection{Teachers' pedagogical reasoning, knowledge, and beliefs}

Pedagogical reasoning is important for insight into the thoughtful and skilful acts of teaching appropriate for a given setting. Shulman (1987) described pedagogical reasoning and action as involving "a cycle through the activities of comprehension, transformation ${ }^{1}$, instruction, evaluation, and reflection" (p. 14). It is necessary to discuss teachers' pedagogical reasoning and action to understand how teachers can develop and apply knowledge in their own teaching contexts.

\subsubsection{Teachers' pedagogical reasoning}

Pedagogical reasoning is characterised as a process of transformation where teachers turn the subject content knowledge into pedagogically powerful forms that fit into

\footnotetext{
${ }^{1}$ Shulman's (1987) term "transformation" is different from Mezirow's (1991) "transformation" which refers to a significant change in understanding or the whole change of assumption and is associated with double-loop learning (Argyris \& Schön, 1974).
} 
student diversity of ability and background (Shulman, 1987). Shulman (1987) developed a model of pedagogical reasoning and action, with an idea of teaching that emphasises comprehension and reasoning, transformation and reflection. The model is composed of activities that teachers do during teaching, including: (1) comprehension of subject-matter knowledge, (2) transformation of subject knowledge into possible instructional representations, (3) instruction (observable performance of various teaching acts), (4) evaluation of students' learning and teacher's performance, (5) reflection on one's own and the class's performance, and (6) new comprehensions (consolidation of new understandings and learning from experience). Then, through reflection teachers can gain new comprehension of teaching, students, and the subject. Therefore, Shulman's model was “an attempt to illustrate reflective practice during the teaching process" (Starkey, 2010b, p. 234). In discussing the conceptualisation of second language teacher education, Richards (2011) referred to teachers' pedagogical reasoning skills as the specialised type of thinking that teachers have and make use of in planning and delivering their lessons. Richards suggested that these special skills allow English teachers to: analyse potential lesson content and identify how to use it as a teaching resource; identify specific linguistic goals for the chosen content; anticipate any possible problems and their solutions; and make appropriate decisions in lesson timing and sequencing and grouping arrangements. Teachers can develop their pedagogical reasoning skills through forms of collaboration. For example, collaboration with more experienced teachers through processes such as shared planning, team teaching, and observation can help less experienced teachers gain an understanding of these teachers' thinking processes (Richards, 2011). These examples suggest that new professional knowledge may be accumulated through critically examining present practice in relation to experience or past practice. Thus, Shulman's model appears to be appropriate for examining teachers' professional knowledge development in that the teachers may gain new comprehension through reflection.

Teachers' pedagogical reasoning can be understood as a process of transformation in which teachers turn the subject content knowledge into pedagogically powerful forms to adapt to students' ability and background, then performing and evaluating students' learning and teachers' performance, reflecting on practice based on evidence, and concluding with the new understandings. It explains the mechanism whereby teachers decide what and how to teach in their teaching context. 


\subsubsection{Teachers' professional knowledge and beliefs}

Teachers' knowledge and beliefs are important determinants of actions. It is difficult to differentiate teachers' beliefs from knowledge (S. M. Bullock, 2011; Calderhead, 1996). However, it is necessary to know what they are and how they can develop and contribute to actions.

\subsubsection{How teachers' knowledge can be developed}

Shulman (1987) identified several categories of knowledge base for teacher knowledge:

- Content knowledge - the knowledge of the subject matter to be taught;

- General pedagogical knowledge - about how to organise classroom activities, give instructions, and so on;

- Curriculum knowledge - the understanding of the course materials and programmes;

- Pedagogical content knowledge - teachers' own special form of professional understanding of how to perform professional teaching such as explicating a concept;

- Knowledge of learners and their characteristics;

- Knowledge of the educational context;

- Knowledge of educational ends, purposes, and values, and their philosophical and historical grounds.

Shulman (1987) noted among these categories, pedagogical content knowledge is distinctive to teachers because it "is the category most likely to distinguish the understanding of the content specialist from that of the pedagogue" (p. 8).

In addition, according to Wallace (1991), teacher knowledge is composed of 'received knowledge,' which is formed through academic education, and 'experiential knowledge,' which is accumulated from classroom experience through reflection. Learning about teaching results from reflection on these two types of knowledge that guide teaching practices (Wallace, 1991). Teachers' learning is commonly characterised as socially constructed through their experience as learners in classrooms 
and schools and in professional teacher education programs, and later as teachers in their work context (i.e., dependent on knowledge of self, students, subject matter, curricula, and setting) (Johnson, 2006). These claims indicate an interrelationship between received knowledge and experiential knowledge and the role of teachers as dynamic users and producers of knowledge. As Johnson and Golombek (2002) discussed, teachers' way of learning "includes an ongoing struggle to articulate an epistemology of practice that characterises teachers as legitimate knowers, producers of legitimate knowledge, and as capable of constructing and sustaining their own professional practice over time" (p. 3). Teachers can be active agents in developing their professional knowledge. Received knowledge and experiential knowledge can be linked and compared through teachers' reflection and shape the way teachers interpret their teaching experiences and refine their practice to suit the classroom context.

Reflection can be a mechanism for teachers to develop knowledge. Abd-El-Khalick (2006) found that reflection impacted the development of teacher knowledge. Learning to teach may require creating opportunities for teachers to develop, make explicit, and articulate the tacit knowledge residing in their experience (Cochran-Smith \& Lytle, 1999). This process can lead to new understanding which derives from their restructuring of existing knowledge, beliefs, and practices (Johnson \& Golombek, 2002). Crookes (2009) claimed, "raising tacit knowledge to consciousness" is central to the concepts of reflective practice (p. 132). In addition, Shulman (1987) noted that the wisdom of practice, which is among four major sources of teacher knowledge (the others being scholarship in content disciplines, educational materials and structures, and formal educational scholarship) is the least codified of all sources of knowledge and provides "reflective rationalization for the practices of able teachers" (p. 11). Wisdom of practice "refers to the full range of practical arguments engaged by practitioners as they reason about and ultimately make judgments and decisions about situations they confront and actions they must take" (Shulman, 2007, p. 560). These ideas suggest that teachers can consciously reflect on practice if they are supported to articulate their practical knowledge, and their pedagogical reasoning skills can be developed in collaboration with others (as mentioned earlier). Thus, in order to develop teachers' knowledge, it may be necessary to help them make practical pedagogical wisdom explicit (i.e., to examine their tacit knowledge). 


\subsubsection{Teacher beliefs}

Beliefs refer to a mental state holding propositions that are accepted as true (Borg, 2001), reside in a tacit form and are not directly observable (Kagan, 1992; Kane, Sandretto, \& Heath, 2002; Nespor, 1987), and can be studied when articulated (Kane et al., 2002). Beliefs are personal constructs based on perception, evaluation and judgment (Clancey, 1997; Nespor, 1987; Pajares, 1992; Roehrig \& Kruse, 2005).

Beliefs in educational settings can be referred to as "one's convictions, philosophy, tenets, or opinions about teaching and learning" (Haney, Lumpe, \& Czerniak, 2003, p. 367). Pedagogical beliefs can be simply defined as beliefs about teaching and learning (i.e., teachers' educational beliefs about teaching and learning) (Ertmer, 2005; Lim \& Chai, 2008). Pajares (1992) recommended that a distinction needs to be made between teachers' broader, general belief systems and their educational beliefs. Furthermore, he claimed that the construct of educational beliefs is broad and includes many diverse aspects, and for research purposes it is necessary to specify what those beliefs are about. According to Pajares (1992), educational beliefs can be:

...beliefs about confidence to affect students' performance (teacher efficacy), about the nature of knowledge (epistemological beliefs), about causes of teachers' or students' performance (attributions, locus of control, motivation, writing apprehension, math anxiety), about perceptions of self and feelings of self-worth (self-concept, self-esteem), about confidence to perform specific tasks (self-efficacy),...about specific subjects or disciplines (reading instruction, the nature of reading, whole language). (p. 316)

Teachers' educational beliefs are a complicated construct. To serve the purpose of this study, some particular form of teacher beliefs such as self-efficacy will be discussed. However, in other cases, teacher beliefs will be used as a generic term as defined by Basturkmen, Lowewen, and Ellis (2004): "statements teachers make about their ideas, thoughts and knowledge that are expressed as evaluations of what 'should be done', 'should be the case', and 'is preferable"' (p. 244), or teachers' pedagogical beliefs (e.g., beliefs about teaching and learning Business English in tertiary education). This definition can be used to examine teacher reflection on practice for change because it shows the interrelationship between teacher beliefs and professional practices.

Beliefs are influential determinants of teachers' classroom practices (e.g., how they define and carry out tasks, organise knowledge and deal with problems) (Donald, 
Blake, Girault, Datt, \& Ramsay, 2009; Kagan, 1992; Pajares, 1992). Beliefs can centrally mediate a teacher's selection and prioritisation of goals and actions (Aguirre $\&$ Speer, 2000). For example, in a study concerning teacher decision-making in the English as a Second Language classroom in three post-secondary institutions, Smith (1991) indicated that "teachers' instructional decisions are centrally influenced by both individually held beliefs about second language learning and teaching as well as experiential knowledge of the ESL classroom" (p. ii). Smith (1996) gave an instance where teachers who appreciated grammatical accuracy emphasised language codes in the design of learning tasks and the curriculum.

Because beliefs are regarded as socially constructed and associated with changes in professional practice, it can be assumed that if there is a mechanism to trigger a change in beliefs, it may stimulate a change in practice. Because teachers' beliefs are shaped by experiences, for beliefs to change it is important that they reflect on these experiences (Pajares, 1992). Three strategies suggested for changes in beliefs are through personal experiences, vicarious experiences, and social-cultural influences (Ertmer, 2005). Ertmer argued that if beliefs are formed through personal experiences, change in beliefs takes place after change in practice and "by helping teachers adopt new practices that are successful, the associated beliefs will also change" (p. 32). Observing others' performances can also contribute to change in beliefs. These two ideas are associated with self-efficacy and vicarious experience which are discussed in section 2.3.1. Then, teachers' practices and beliefs are continually influenced by their ongoing teaching experiences and by surrounding people's values and opinions (Ertmer, 2005). These ideas suggest that if teachers' reflection is promoted, it may bring about changes in beliefs and subsequent action, which in return can trigger reflection on beliefs. The trend in research into teachers' beliefs stemmed from a common view that to gain insight into teaching, it is necessary to understand what teachers think, know, believe, and do (Borg, 2003; D. Bullock, 2011). Thus, this area is an important issue to consider in the thesis.

\subsubsection{Summary}

Teachers' knowledge and beliefs are closely associated with academics' pedagogical reasoning and action. These affect their ability to carry out teaching tasks such as transforming content knowledge into appropriate instructional representations and make changes for effective classroom practice. In order to enhance teachers' learning 
about teaching, it may be necessary for teachers to engage in the process of reflection whereby their knowledge and beliefs, together with attitudes, values, and experience are challenged, refined, or even changed, which helps raise to consciousness the nature of their personalised theories of practice. To execute changes in practice, academics may also need to reflect on the way they transform knowledge into teachable forms in the classroom (the role of reflection in professional learning is discussed in section 2.4.1.2). Therefore, a process which makes teachers aware of how their knowledge and beliefs shape their understanding of teaching and how they make teaching decisions in practice may influence and provide insights into teachers' process of pedagogical reasoning and action.

\subsection{Self-efficacy and autonomy: motivation for actions}

As discussed above, teachers' beliefs influence teaching behaviours. However, the above explanation does not provide insight into what motivates teachers to take actions for teaching improvement. Based on difference in goals for actions, Ryan and Deci (2000a) distinguished "intrinsic motivation, which refers to doing something because it is inherently interesting or enjoyable" from "extrinsic motivation, which refers to doing something because it leads to a separable outcome" (p. 55). Intrinsic motivation reflects human inclination to learn and assimilate and is "the inherent tendency to seek out novelty and challenges, to extend and exercise one's capacities, to explore, and to learn" (Ryan \& Deci, 2000b, p. 70). This implies that being intrinsically motivated entails being willing to take actions. Knowles et al. (2005) suggest that intrinsic motivation is more powerful for adult learning. In addition, a number of studies (e.g., Canrinus, Helms-Lorenz, Beijaard, Buitink, and Hofman, 2012; Skaalvik and Skaalvik, 2014) suggested that self-efficacy and autonomy contribute to intrinsic motivation. Therefore, in carrying out an intervention process for academics to improve practice, it may be necessary to consider whether it develops their sense of self-efficacy and autonomy.

\subsubsection{Teacher self-efficacy}

Self-efficacy, from the standpoint of social cognitive theory, can be generally viewed as one's personal beliefs in one's own capabilities (Bandura, 1977a). Self-efficacy is defined as "people's judgments of their capabilities to organize and execute courses of action required to attain designated types of performances" (Bandura, 1986, p. 391). It 
denotes an internal attribution where one sees oneself as the cause of an action, refers to future behaviours, and is a predictor of actual behaviours (Schwarzer \& Hallum, 2008). Bandura (1977a, 1997) identified four possible sources of people's beliefs about their personal efficacy:

- Mastery experiences: are indicators of capability (Bandura, 1997) and are enhanced when people perceive their performance as successful. Then, enhanced self-efficacy leads to their expectations of successful future performance. Otherwise, efficacy decreases if people perceive their practice as a failure.

- Vicarious experiences: are gained when observing others' performance and contribute to the enhancement of self-efficacy through competency transmission and comparison with others' goal achievement (Bandura, 1997). The impact of the modelled performance on the observer's self-efficacy depends on the extent to which the observer identifies with the observed (Tschannen-Moran \& Hoy, 2007). Modelled performances with clear outcomes can bring about more efficacy information (Bandura, 1977a). When the observed person performs well, the observer's self-efficacy sometimes rises. When the model and the observer have salient differences in aspects such as the level of experience, training, gender, or race, watching a very competent performance may not enhance the observer's self-efficacy (Tschannen-Moran \& Hoy, 2007).

- Verbal persuasion: relates to conversations about people's performance and opportunities for success with others such as colleagues. When people receive each other's encouragement or persuasion in their capacities to perform tasks successfully, they will probably attempt greater sustained effort (Bandura, 1986).

- Physiological arousal: also contributes to a sense of efficacy. For example, feeling pleased from successful performance may enhance teachers' sense of efficacy; on the contrary, feelings of stress or anxiety relating to a fear of losing control may result in lower self-efficacy (Tschannen-Moran \& Hoy, 2007).

Self-efficacy is important because it impacts on one's feeling, thinking, and actions. Growing research supports Bandura's (1997) social cognitive theory that academics' self-efficacy is related to their classroom behaviour and affects their attempts in teaching, aspirations, and persistence to deal with difficulties (e.g., Tschannen-Moran 
\& Hoy, 2001; Schwarzer \& Hallum, 2008; Mansfield \& Woods-McConney, 2012). Different levels of self-efficacy can increase or hinder one's motivation to act. Teachers with high self-efficacy are believed to be more resilient in their teaching and likely to try harder to help students explore their capability whereas those with low self-efficacy are less likely to make an effort to meet student needs (Pendergast \& Garvis, 2011). Ryan and Deci (2000a) suggested that when three innate psychological needs (i.e., competence, autonomy, and relatedness) are satisfied, self-motivation and mental health will be enhanced. A sense of self-efficacy is associated with feeling of competence. Feeling competent helps maintain intrinsic motivation and psychological well-being (Gagne \& Deci, 2005; Mansfield \& Woods-McConney, 2012; Pendergast $\&$ Garvis, 2011). Interpersonal interactions such as feedback that bring about "feelings of competence during action can enhance intrinsic motivation for that action because they allow satisfaction of the basic psychological need for competence" (Ryan \& Deci, 2000a, p. 58). In other words, self-efficacy contributes to intrinsic motivation; thus, for teachers to take actions to improve practice, it is necessary to promote their sense of self-efficacy.

\subsubsection{Teacher autonomy}

Little (1995) describes autonomous teachers as having a strong sense of personal responsibility for their teaching exercised through continuous reflection, control, and freedom in the teaching process. Teacher autonomy is also conceptualised as an ability to develop appropriate teaching skills, knowledge and attitudes for themselves as teachers, in collaboration with others (Smith \& Erdoğan, 2008; Yan, 2010). Sometimes, it is referred to as teachers' ability to influence their teaching life (such as control over scheduling, curriculum, textbooks, and instructional planning) and freedom to make decisions (Benson, 2000; Short, 1994). These definitions appear to relate autonomy to teachers' capacity to engage in self-directed teaching.

In discussing the dimensions of teacher autonomy, Smith and Erdoğan (2008) identified six characteristics:

- Self-directed professional action,

- Capacity for self-directed professional action,

- Freedom from control over professional action, 
- Self-directed professional development,

- Capacity for self-directed professional development,

- Freedom from control over professional development (pp. 84-85).

Yan (2010) also indicated that a teacher's attitude is another crucial element in teacher autonomy. Yan argued that it is necessary to make "a distinction between capacity for and/or willingness to engage in self-direction and actual self-directed behaviour" ( $p$. 175). For example, some Chinese academics have the capacity to engage in selfdirected activity but are not willing to do so for the sake of personal responsibility (Yan, 2010). Thus, teachers' attitudes may determine whether they perform actual selfdirected behaviours when autonomy is facilitated.

The interpretations of the dimensions of teacher autonomy may be useful for research into reflective practice. As Lamb (2008) indicated, in relation to professional action or teaching, the extent of teachers' capacity to improve teaching through effort (through reflective or research-oriented approaches) and freedom to teach in their preferred way denote conceptualisations of teacher autonomy. With regard to teacher learning, Lamb stated that the interpretations of teacher autonomy emphasise teachers' capability of making decisions according to their professional learning needs, or the freedom to do so. Lamb suggested that research is necessary to investigate the manner in which teacher autonomy (and interventions designed to enhance teacher autonomy) influences learning outcomes, motivation and professional satisfaction. Considering these dimensions together with teachers' attitudes is useful for interpreting teachers' actions in teaching and learning about teaching.

Teachers' sense of autonomy can be the driving force of their actions. Teacher autonomy may relate to teachers' freedom for aspirations, teaching methods, and educational strategies that align with their own teaching beliefs and values (Skaalvik \& Skaalvik, 2014). Empirical evidence from Skaalvik and Skaalvik's research showed that teachers' autonomy is positively associated with their engagement in teaching and job satisfaction. However, they argued that high autonomy may bring about different outcomes depending on whether teachers' mastery expectations (i.e., self-efficacy one dimension of self-perceived competence) are high or low. Teachers with high mastery expectations may try new ideas and make changes to practices, which may facilitate personal learning and development. Meanwhile, those with low mastery 
expectations may view autonomy as a chance to conceal their self-aware weaknesses and retreat from challenges, which may hinder their personal learning and growth. Moreover, in addition to feelings of competence (as mentioned earlier), a sense of autonomy can help maintain intrinsic motivation (Gagne \& Deci, 2005). Thus, in order to motivate teachers to take actions for changes in teaching, it may be necessary to promote their autonomy which can be exercised through reflection.

\subsubsection{Summary}

Teachers' self-efficacy and autonomy have been believed to be associated with teachers' changes in practice because they can contribute to intrinsic motivation. Examining sources of self-efficacy such as vicarious experiences and verbal persuasion implies that the use of modelling and feedback to teaching (i.e., external perspectives on teaching) may be a way to make teachers aware of their beliefs in their capacity to execute an action. Examining the definition and dimensions of teacher autonomy suggests reflection can be a mechanism for teachers to become aware of their personal responsibility, control and freedom in teaching. Therefore, when implementing an intervention process to promote academics' reflection and changes to practice, it is necessary to take into account their sense of self-efficacy and autonomy.

\subsection{Teacher reflection and reflective practice}

\subsubsection{Reflection contributing to professional learning}

Reflection has been considered as a process that contributes to professional learning. It can be a mechanism that underlies teachers' understandings and actions. This section presents the conceptualisation of reflection and reflective practice, how it contributes to teachers' learning, and conditions for effective reflection.

\subsubsection{Conceptualising reflection and reflective practice}

The term reflection is used with many different meanings. For example, LaBoskey's (1993) robust review of the literature indicated that there is inconsistent use of the term reflection among the theoreticians, researchers, or teacher educators. Thus, it is necessary to discuss the definition of reflection and identify one for use in this thesis. 
The notion of reflection in teaching was conceptualised by Dewey $(1997)^{2}$ at the beginning of the $20^{\text {th }}$ century. He regarded reflection as an active and deliberative cognitive process (Hatton \& Smith, 1995; Pedro, 2006). With a distinction between routine action and reflective action, Dewey's discussion on reflective thought indicates that a truer and more reasonable conclusion about reality is likely to be evidenced in one's thought if there is doubt, inquiry, and collection of evidence about the nature of things. Thus, Dewey defined reflective thought as "active, persistent, and careful consideration of any belief or supposed form of knowledge in the light of the grounds that support it, and the further conclusions to which it tends" (Dewey, 1997, p. 6).

Following the definition, Dewey stated the components of reflective thinking: (1) "a state of perplexity, hesitation, and doubt", in which a problem challenges and makes one's belief questionable; and (2) "an act of search or investigation directed toward bringing to light further facts which serve to collaborate or to nullify the suggested belief" (p. 9). Dewey indicated that past experience and prior knowledge as well as thinking skills (maintaining the state of doubt and carrying on the systematic and prolonged questioning process) are the essentials of reflective thinking. It appeared that Dewey dealt with reflection in the setting of hypothetical-deductive problemsolving (Hafler, 2002; Mezirow, 1991). The distinctive feature of Dewey's concept of reflection is that one sees the relation between the situation one is in and the desired goal. What counts most in the educative process is to train a mind to be capable of identifying problems and skilled in methodologically critical thinking and solution. However, Dewey's approach "tends to over-emphasise the procedures of logical thinking” (LaBoskey, 1993, p. 26). Dewey's emphasis on prior knowledge and thinking skills as key mediators of reflective thinking may imply that the practitioner with the necessary prerequisites will be able to reach a satisfactory conclusion without external supportive sources.

A second scholar who contributed to the application of reflection in education is van Manen (1977). In discussion on teachers making practical use of knowledge about curriculum, van Manen outlined three levels of reflectivity of deliberative rationality: the technical-practical, the interpretative/contextual, and the critical. At the first level, teachers" rationality of decisions stems from "the principles of technological progress - economy, efficiency, and effectiveness" (p. 226). On this level, the practical

\footnotetext{
${ }^{2}$ Reprint of Dewey's (1910) How we think
} 
significance of the knowledge constructed through a cognitive activity means that teachers technically apply educational knowledge and basic curriculum principles to achieve a given objective. The outcomes for reflection at this level may involve effective selection and implementation of lessons to achieve objectives, and there may be a transition into linking theory development to practice and identifying the appropriateness of activities and objectives (Taggart \& Wilson, 2005).

At the second and higher level - the interpretive/contextual - the practical significance, according to van Manen, refers to "the process of analysing and clarifying individual and cultural experiences, meanings, perceptions, assumptions, prejudgments, and presuppositions, for the purpose of orienting practical actions" and focuses on "an interpretive understanding both of the nature and quality of educational experience, and of making practical choices" (pp. 226-227). In other words, educational experience and practical choices are examined within some interpretative framework; that is to say, the alignment of theory and practice (Argyris \& Schön's (1974) espoused theory versus theory-in-use) and the adequacy and coherence of one's theory are considered in the reflection process at this level (Jones, 2007). This kind of reflection is similar to the one occurring in the "double-loop learning" described by Argyris and Schön (1974, p. 18) or "theory building" described by Korthagen and Lagerwerf (2001, p. 183). Korthagen and Lagerwerf described three levels of learning: (1) gestalt formation where one's action tendencies are based on previous experience with similar situations by which one's needs, thoughts, feelings, values, and meanings are unconsciously stimulated, (2) schematization where conscious actions are taken with a need for clarifying a situation and where concepts, characteristics, and principles of practice may be developed, (3) theory building where one forms the logical structuring and reassessing of the relations of the elements in the schema or several schemata that are connected into one coherent theory. It can be inferred that learning at the third level, or double-loop learning (Argyris \& Schön, 1974), leads one to restructure core beliefs. At the third and highest level of van Manen's (1977) system of reflectivity - the critical, reflection is then focused on social, political, and ethical issues of teachers' practice. At this level, practitioners think deeply about ethical and political issues relative to teaching planning and implementation, being "concerned with worth of knowledge and social circumstances useful to students without personal bias" (Taggart 
\& Wilson, 2005, p. 5). At this level of reflection, practitioners question moral and ethical issues relating to various teaching practices.

The term 'critical' causes confusion, so it is necessary to differentiate the two meanings it refers to. Taggart and Wilson indicated that van Manen's term 'critical' is comparable to the term 'dialectical' used by Grimmett, Mackinnon, Erickson, and Riecken (1990) and Lasley (1992). In order to avoid confusion, the meaning of the word 'critical' needs to be elaborated: the first sense is associated with the process of critiquing an idea or a practice, and the second one relates to critical theory (e.g., van Manen, 1977) (Jones, 2007). Consequently, when referring to the first sense, critical will be used. When referring to the second sense, the word dialectical as employed by Taggart and Wilson (2005) is used instead.

There are critiques on van Manen's categorization of reflection. LaBoskey (1993) commented that van Manen's hierarchical conceptualisation devalues the technicalpractical and interpretive, so she suggested that these categories should be referred to as content of reflection rather than levels of reflection and that they are of equal importance. LaBoskey suggested that it is possible to reflect on issues "within or across categories" (p. 26). Much reflective content is found related to the first two levels of reflection in both expert and novice teachers (McIntyre, 1993; Sparks-Langer \& Colton, 1991). Sparks-Langer and Colton indicated that most designers of teacher education programmes at universities found it relatively easy to promote technicalpractical reflection but more difficult to achieve dialectical reflection. Many novice teachers are believed to exercise reflection at a technical level based on a lack of schemata in solving educative problems (Taggart \& Wilson, 2005). In discussing the cognitive element of reflection, Sparks-Langer and Colton (1991) indicated that because of lack of experience, novices have less developed schemata - the organised structures that portray information organised into a network of related facts, concepts, generalisations, and experiences. These schemata constitute one's understanding of the world and allow information to be stored and accessed quickly. The lack of schemata may require novice teachers to take action to improve practice; therefore, they use reflection as a way to gain knowledge for practice. Dialectical reflection, whereby teachers interrogate moral and ethical issues relating to various teaching practices, is difficult to achieve. Reflection needs confrontation (with sensitive balance of challenge and support) and collaboration (Convery, 1998). Taggart and Wilson 
(2005) suggested that "facilitators working with practitioners functioning at a dialectical level should provide a forum to assist them in deciding worthiness of actions and analysing curriculum approaches, case studies, conventional wisdom, and technocratic approaches" (p. 5). A distinctive characteristic of dialectical reflection is risk taking in the form of peer review and self-assessment, which helps teachers achieve self-efficacy and self-actualisation and thereby reconstruct action situations as a means of reviewing the self as teacher and challenging previously held assumptions (Taggart \& Wilson, 2005). The evidence means that the reflector's new understandings fundamentally depend on dialectical reflection that requires examination of assumptions and perspectives of the world, and these are not easily recognised without confrontation deriving from external sources.

Another scholar who enriched the discussion on reflective practice for teachers' professional development is Donald Schön. According to Schön (1983), professionals frame and reframe problems in practice and refine their actions. Schön (1983) employed the notion of reflective practice which incorporates rational examination of one's own experiences in applying knowledge to practice. Reflection-in-action can be viewed as a reflective form of knowing-in-action which is ordinarily tacit. Schön (1983) assumed that:

competent practitioners usually know more than they can say. They exhibit a kind of knowing-in-practice, most of which is tacit.... Indeed, practitioners themselves often reveal a capacity for reflection on their intuitive knowing in the midst of action and sometimes use this capacity to cope with the unique, uncertain, and conflicted situations of practice. (1983, pp. viii-ix)

When a professional faces a problem, the assumed structure of tacit knowing-in-action is critically questioned by 'reflection-in-action' (i.e., in the middle of an activity) that leads them to a re-examination of the situation to "restructure strategies of action, understandings of phenomena, or ways of framing problems" (Schön, 1987, p. 28). Schön's model of the reflective practitioner puts weight on practitioners' intuitive reshaping of events as a form of professional capability through 'reflection-in-action' and rejects the technical rationality and 'hypothetical-deductive' models of thinking and decision-making (Ecclestone, 1996). Such rejection can be illustrated with this critique: unreflective teachers are inclined to accept the everyday reality in their school and try to find the most effective and efficient means of problem-solving that has been 
collectively validated and prescribed (Harrison, 2008; Zeichner \& Liston, 2011). Schön argued that the technical rationalist approach, a positivist version of enquiry, did not reflect the confusing contexts of professional decision-making where technical solutions did not work with the complex/indeterminate problems. Schön (1987) also discussed another type of reflection - 'reflection-on-action' which comes after an activity and involves the same process as 'reflection-in-action.' $\mathrm{He}$ argued that professionals were different from mere technicians in their capacity to reflect about the moral and ethical aspects of their practice in messy, confusing contexts and their ability to carry out this reflection spontaneously and intuitively in action. Schön's idea on reflective practice has had a great influence on professional development programmes (Ecclestone, 1996; Jones, 2007).

The term reflection has caused confusion because of its multiple interpretations. In synthesising many different conceptualisations of reflection, Korthagen (2001) reached a common understanding of reflection that "reflection is the mental process of trying to structure or restructure an experience, a problem or existing knowledge or insights" (p. 58). However, Jones (2007, p. 51) argued that if reflection is to facilitate the development of professional expertise and capability, the process needs to involve "a more specific focus on practice and the outcome on practice that results" in addition to the mental structuring and restructuring described by Korthagen (2001). This focus that reflection aims at is discussed in adult learning.

Reflection characterises dynamic adult learners and emphasises application of knowledge to practice and its outcomes. First, research on adult learning depicts adult learners "as autonomous individuals capable of identifying their own learning needs and planning" (MacKeracher, 2004, p. 23). From a standpoint of adult learning theory, Knowles (1993) argues that reflection:

...effectively challenges the thinking about events, circumstances and philosophies which constitute and value the status quo... (it) is seen as a means of emancipation and empowerment, a vehicle for allowing both teacher educators and teachers to take control of environments and circumstances in which they work and students learn. (pp. 82-83)

This belief in the importance of autonomy, independent thinking, and challenge to existing forms of knowledge and practice is distinctive of andragogical approaches to adult education and tertiary education (Ecclestone, 1996). Self-directedness is one of 
the factors contributing to adults' motivation to learn, and life experiences are used to develop procedures for critically examining the past, interacting in the present, and anticipating the future (MacKeracher, 2004). Adults gain experience and new knowledge over their lifetime and bring this to their current learning. These ideas suggest that reflection supports teachers to gain the awareness and understanding of their own thought processes about how they apply their learning to current practices and planning for the future.

It is commonly agreed that reflection denotes the ongoing process in which the practitioner, from an importantly influential system of beliefs, critically considers their present and past practices as a way to gain knowledge and understanding to improve practice (Buysse, Sparkman, \& Wesley, 2003; Jones, 2010). It is also necessary to distinguish the terms related to the concept of reflection. Reflection can occur before, during, or after an action. That is to say, the first case - 'reflection-for-action' - is based on previous experience. Then, reflection concurrent with an action is called 'reflection-in-action' (Schön, 1983, 1987). Finally, reflection coming after an action 'reflection-on-action' (Schön, 1983, 1987) - represents the most common conception of reflection (McAlpine \& Weston, 2000). When reflection on practice is referred in the study, it means reflection after actions.

The discussion of the conceptualisation of reflection supports LaBoskey's (1993) perspective that van Manen's three levels of reflection (the practical, the interpretative, and the dialectical) are equally crucial to teachers' reflection. Ideas on reflection and adult learning provide the understanding of the context of learning through which problems are defined and solved, and practices are evaluated. Mezirow (1991) proposed that reflection can be on the content, process, and premise of one's belief or understanding of a situation. Mezirow (2003) indicated the need "to make explicit the differences involved in reflecting on the content, process, or premises of problem solving" (p. 202).

The first one is about problem-framing with a focus on the important variables in the situation. For example, an English language teacher can reflect on comments by a fellow teacher that suggests it is not worth the effort to use games in the classroom because it does not aid students' learning. The second involves examining the strategies and procedures of problem-solving and assessing one's efficacy in performing actions. For example, the teacher can reflect on how he/she feels about 
using games in the classroom and how confident he/she feels he/she could implement games which are useful for students' learning. Content and process reflections help practitioners to make conscious judgments on the procedure and the likely success of their actions in thoughtful actions where reflection may or may not occur.

The third - premise reflection - involves the questioning of one's beliefs and assumptions (Mezirow, 1991). For example, the teacher with a prior belief that games did not aid to student learning could reflect on his belief after realising that students were so tired and stressful with other subjects that they could not engage in his English lesson. The teacher then tried using games and realised that students became more active and engaged in learning. Thus, by questioning his belief whether games are necessary for student learning (i.e., whether a classroom with lack of stress is essential for learning), trying them, and recognising the students' enhanced engagement, the teacher changed his belief and decided to continue with games. Mezirow clarified that premise reflection "might involve an assessment of the validity of norms, roles, codes, 'common sense,' ideologies, language games, paradigms, philosophies, or theories" (p. 105). In other words, premise reflection or critical reflection ('critical' in the first sense mentioned above) refers to "challenging the validity of presuppositions in prior learning" (Mezirow, 2003, p. 207). This also aligns with Brookfield's (1995) idea of “hunting assumptions" (p. 3). Mezirow's premise reflection (i.e., 'critical reflection' as used by many researchers) may focus on van Manen's technical, interpretive and critical/dialectical levels. In other words, through such critical reflection (i.e., hunting/exploring assumptions about practice), teachers will be able not only to examine the technical issues of teaching, but also look interpretively and dialectically at aspects of practice. This is the process whereby practitioners make explicit their tacit knowledge upon which their practice is based. In short, reflection on content, process, and premise refers to an assessment of the what, how, and why of perceptions, thought, feelings, or actions. Mezirow's (1991) framework is used for analysing academics' reflection in this thesis because it does not devalue the technical-practical as in van Manen's conceptualisation.

\subsubsection{How reflection may contribute to learning}

Reflection has been advocated both as a process and as a tool to examine thoroughly and gain insights into teaching (Marcos, Sánchez, \& Tillema, 2008; McAlpine \& 
Weston, 2000). Reflection plays an essential role in teacher learning, as Mezirow (1991) argued, for its importance in adult learning:

Reflection is the central dynamic in intentional learning, problem solving and validity testing through rational discourse. Intentional learning centrally involves either the explication of the meaning of experience, reinterpretation of that meaning, or application of it to thoughtful action. (p. 99)

Loughran (1995) suggested that reflection should be viewed by teachers as part of their own process of learning about learning and teaching, which then will bring them the understanding of their context-specific pedagogy and the ability to make decisions on appropriate application of knowledge in their own practice. The reflective process focuses on "hunting assumptions" (Brookfield, 1995, p. 3), examining practices from as many unfamiliar perspectives as possible to have appropriate guides for action (Brookfield, 1995). Reflection through questioning and investigating these assumptions relating to teaching and learning can lead to a developing understanding of professional practice (Harrison, 2008). In addition, teachers' ability to apply ethical norms to the objectives and processes of teaching and learning would determine the quality of their reflection (Valli, 1997); therefore, they should consider the worth of knowledge, social consequence, and defence of choices (Taggart \& Wilson, 2005). In other words, professionals need to judge the worthiness of their actions that align with their goals and the ethical values of actions. Pollard (2005) stated, "reflective teaching implies an active concern with aims and consequences as well as means and technical competence" (p. 15).

Reflection may result in single-loop and/or double-loop learning (Argyris \& Schön, 1974): refining practices to solve problems and/or restructuring one's beliefs and values or attitudes, and theories of practice. Relating to long-term professional development, Jones (2007) indicated that the reflection process is a means of informing and improving practice but emphasised that it may be at times used for evaluating one's practice rather than a deliberative problem-framing and solving process. In other words, teachers through reflection are able to develop new premises of practice which will foster new and improved practices (Jones, 2007; Korthagen, 2001).

Reflection should be viewed as a mechanism that supports teachers' ongoing professional development whereby they: build their own knowledge; clarify and 
elaborate underlying assumptions, predispositions of classroom practice, and the outcomes of actions; and question the moral and ethical issues of teaching practices.

\subsubsection{Conditions for effective reflection}

For reflection to be effective, teachers require specific attitudes and skills or competence. Appropriate attitudes for reflection may maximise the effectiveness of reflection. As Moore and Asay (2013) discussed, attitudes - values expressed within social contexts - are states of readiness that will influence a person's response to situations and will change with experience and education. Dewey (1997) identified three necessary attitudes for a reflective practitioner - open-mindedness to alternative possibilities, responsibility in judging the outcomes, and whole-heartedness to take actions from ideals. In a framework for teacher reflection, Colton and Sparks-Langer (1993) suggest four qualities of reflective decision makers: efficacy (belief in one's ability to make a difference), flexibility (ability to take other perspectives to find new meanings and interpretations), social responsibility (caring about and contributing to social causes), and consciousness (awareness of one's own thinking and decision making). Grant and Zeichner (2000) emphasised the importance of 'complete openness of mind' because "reflective teaching involves a balance between the arrogance that blindly rejects what is commonly accepted as truth and the servility that blindly receives this "truth"' (p. 35).

In addition, the development of the capacity for reflection and reflective practice has been considered an important determinant of the emergence of a capable reflective teacher. Wheatley (2006) emphasised the importance of capacities for reflection:

It's hard to look at modern life and see our capacities for reflection or meaningmaking... all changes results from a change in meaning.... We change only if we decide that the change is meaningful to who we are. (p. 179)

This capacity involves the ability to build a sound knowledge base for reflection and the necessary skills for effective actions, without which reflection will not yield improved practice (Jones, 2007).

An effective reflective teacher has the ability to make a distinction between reflection and rationalisation of practice, which is crucial to the development of teachers' professional knowledge (Loughran, 2002). Loughran suggested that rationalisation may disguise itself as reflection, by which he meant that rationalisation of practice is 
apparent when a problem is dominated by existing perspectives and is not examined from varied viewpoints. Contrasting effective reflection with rationalisation of practice, Loughran asserted that reflection is effective when it facilitates a teacher's meaning-making of a situation in ways that enhance understanding in order that the teacher comes to examine and understand the context of practice from a variety of viewpoints. To examine practice through many different lenses, reflective teachers need "competence in methods of evidence-based classroom enquiry" (Pollard, 2005, p. 14). For instance, collaboration and dialogue with colleagues can lead to the enhancement of reflective teaching, professional learning, and personal fulfilment (Pollard, 2005).

In summary, reflection and reflective practice provide teachers with a means of building self-knowledge and understanding of teaching and learning, through which they are able to constantly examine and refine practice with attention to goals and outcomes of actions, thus making a valuable contribution to their ongoing process of learning about teaching. These aforementioned prerequisites for effective reflection suggest several implications for programmes that enhance teachers' reflection and teaching improvement. Programmes for improving teachers' practice may need to facilitate the development of their qualities/characteristics, capacity, and particular disposition for reflection. In addition, because effective reflection and professional learning require teachers to seek recourse to external sources of information on teaching such as students' and colleagues' lenses in order to examine their practice, forms of feedback collection and collaboration might be needed.

\subsubsection{Academics' critically reflective lenses}

Reflection can occur as a solitary activity and/or a collaborative activity. A critical examination of teaching can be done individually through solitary framing and reframing teaching events or, more publicly, by inviting an observer to experience them, using another set of lenses (Harrison, 2008). However, Convery (1998) indicated that solitary reflective practice did not lead to his self-awareness of inhibiting defensive behaviours, so he emphasised the importance of collaboration. Reflection and reflective practice can be facilitated through a specific supervisory, coaching or critical friend relationship (Convery, 1998). Thus, external sources can mediate teachers' effective reflection by encouraging the questioning of assumptions, thought 
processes, values, prejudices and habitual actions, and attitudes for understanding of practice and finding necessary strategies for improvement.

In order to gain insight into practice, reflective teachers need a variety of lenses (i.e., multiple viewpoints) to effectively reflect on practice. For reflection to be a genuine window into the world of practice, it is important that reflection facilitates teachers' questioning their taken-for-granted assumptions and examination of their practice through others' eyes (Bolton, 2010; Brookfield, 1995; Forde, McMahon, McPhee, \& Patrick, 2006; Loughran, 2002). This means teachers' reflection should be put under scrutiny for maximum effectiveness (Bolton, 2010; Harrison, 2008; Pollard, 2005). As aforementioned, the four lenses for academics to examine teaching include autobiographies as teachers and learners, students' eyes, colleagues' experiences, and theoretical literature (Brookfield, 1995), which are associated with the processes of self-reflection, student feedback, peer professional conversation, and reading of the relevant literature respectively. Examining practice through these lenses can highlight distorted aspects of teachers' assumptions and allows them to enhance their understanding and further define assumptions. For examples, teachers may need to collect external information about their practice (e.g., student feedback, colleague feedback, consultative feedback from experts, etc.) to examine the similarities and differences between their and others' perceptions of their practice. Though the four lenses are valuable to teacher reflection, students' and colleagues' lenses are viewed as external sources of evidence on practice and may generate confrontation that triggers reflection. Therefore, they are the focus in this study situated within the broader spectrum of evidence-based inquiry into teaching practice and are discussed now.

Students' eyes: Gaining the understanding of teaching and learning through students' eyes is, for Brookfield, "of utmost importance" to responsive and good teaching (p. 35). Examining practice through students' eyes can lead to responsive teaching. Gaining a deeper understanding of what students are experiencing helps teachers consciously confirm or challenge their pedagogic assumptions and check if students understand their intended meaning. Brookfield believed that it is hard for teachers to teach well without knowing students' perspectives on methodological choices; without knowing if these work for students, teachers may risk making ill-informed and inappropriate methodological choices. One problem with student feedback is their 
hesitation in offering honest critiques for fear of negative results, so providing anonymity can be a good solution (Brookfield, 1995).

Colleagues' experiences: For Brookfield, although critical reflection can be a solitary activity, "it is ultimately a collective endeavour" (p. 36) (Boud, Cressey, and Docherty (2006) also mentioned this idea of a collective approach to reflection in the discussion of productive reflection at work). Critical dialogues with colleagues can help teachers learn from others' experience and perspectives. By getting information from colleagues' observations, feedback, or critical dialogues, teachers can examine the hidden aspects of their practice. For instance, as their colleagues describe the same problem they have faced, teachers are able to check, reframe, and broaden their own theories of practice (Brookfield, 1995). These dialogues can create a community of learning where colleagues serve as a mirror to reflect on practice and as a source of possible pedagogical strategies.

Students and colleagues' perspectives appear to be valuable external sources which may be necessary for teachers to reflect on teaching in order to improve it. These reflective lenses on teaching can lead teachers to pedagogical reasoning whereby they reflect upon their own classroom practice as they deliberately scrutinise their actions and gain an understanding of teaching and learning. Therefore, programmes that aim to enhance teachers' reflection and reflective practice and positive changes in teaching practice may need to consider using students' and colleagues' lenses.

\subsection{Student feedback}

Feedback is conceptualised as "information provided by an agent...regarding aspects of one's performance or understanding" (Hattie \& Timperley, 2007, p. 102). Students' feedback in general refers to their perceptions on the educational service they receive such as the teaching and learning process, the learning environment, the support facilities, and other external aspects of being a student (Harvey, 2003). Collecting student feedback on teaching can be a way of understanding the complexity of the learning experience from students whose learning is the objective of a teacher's teaching (Belluigi, 2013). This use of student feedback indicates its role as a lens for teachers' reflection and reflective practice. Students are important stakeholders, and considering their feedback is important to any quality process in tertiary education (Harvey, 2003). With a view that the key purpose of feedback is to improve teaching 
performance, this section looks at formative student evaluations of teaching (SETs). It is necessary to discuss what SETs are used for, what value they have for academics' reflection and reflective practice, how they are collected, what challenges they face, and whether SET can be effective as the only source of information for academics' reflection.

\subsubsection{Purposes of student feedback in tertiary education}

SETs (as a form of collecting student feedback) have been used in many universities (Kember, Leung, \& Kwan, 2002; Smith, 2008; Yao \& Grady, 2005; Yin, Lu, \& Wang, 2014). Three interrelated justifications given for the use of SETs includes their contribution to teaching improvement, appraisals, and institutional explicit requirements of quality reviews (Harvey, 2003; Kember et al., 2002). The purposes of SETs may include: diagnostic feedback facilitating the development and improvement of teaching; research data that will bring further improvements to units, courses, curriculum and teaching; data used for administrative decision-making; a source of information for current and potential students' choices related to the institution and programmes; and as a measure for judging quality of performance tied to external funding formulae (Bennett \& Nair, 2010; Kember et al., 2002; Marsh \& Roche, 1993; McKeachie, 1997; Richardson, 2005; Rowley, 2003; Seldin, 1989). SETs can be used for summative and formative purposes. It should be noted that although these uses and purposes of SETs have been defined in developed countries, they are not well defined in countries where SETs are relatively new (Nair \& Mertova, 2013). SETs have been accepted as a means of obtaining student feedback on the quality of tertiary teaching (Ballantyne, Borthwick, \& Packer, 2000; Seldin, 1993). The use of SETs in tertiary education environment has been under debate around their perceived value and validity (Marsh \& Roche, 1997). However, the developing focus on quality, accountability, and the promotion of teachers' reflective practice has boosted the use of SETs to evaluate or provide feedback on teacher performance (Moore \& Kuol, 2005). A key purpose of student feedback is to improve teaching performance (Marsh \& Roche, 1993; Reid, 2010; Seldin, 1989). There are a number of studies employing SETs for improving teaching such as Winchester and Winchester (2011), Bullock (2003), and Aultman (2006). These show that besides other purposes, SETs can be used formatively for academics to reflect on and improve their teaching practice. However, SETs that contain only Likert scale questions seem not to contribute much 
to teachers' improvement (Smith, 2008). Thus, mid-term formative SETs tend to have both Likert scale questions and open-ended questions. For example, the University of Illinois at Urbana-Champaign employed online student ratings (which was developed by teachers who can choose both forced-choice and open-ended items from the existing online evaluation item bank) to collect midterm formative feedback besides end-of-term summative SETs (Bullock, 2003). Another example is that Aultman (2006) designed her mini-SETs with three sections: the first for students to write down their questions, the second for their ratings on four different areas, and the third for students' comments on ways she could improve teaching. SETs of this type can be beneficial to focus on teachers' needs. SETs can help teachers to critically examine their teaching and focus on areas that need improving. SETs with appropriate design for formative purpose can be a lens for teachers' reflection as discussed in the following section.

\subsubsection{Formative SETs triggering reflection}

Previous studies have shown that formative SETs are one possible source of information that can be used to promote reflection (Beaty, as cited in Winchester \& Winchester, 2011). Ramsden (2003) also emphasised the formative purpose of SETs:

Evaluation is a way of understanding the effects of our teaching on students' learning. It implies collecting information about our work, interpreting the information and making judgments about which actions we should take to improve practice. (p. 209)

Formative SETs are used for academics' teaching improvement and personal development. These SETs focus on academics' desired goals and the needs of the institution (Winchester \& Winchester, 2011). Formative SETs can allow academics certain autonomy to reflect on particular aspects of practice, thus promoting their intrinsic motivation to further investigate their teaching. When academics are motivated to improve practice, they are likely to use SETs for improving teaching (Yao \& Grady, 2005) because students, as "the ultimate raison d'être" for academics and their teaching, are the first and most essential source of feedback (Kelchtermans, 2009, p. 262). Early-term student feedback makes teachers aware of students' perceptions and reflect on their teaching (Penny, 2004). 


\subsubsection{Conditions for effective SETs and collecting TT-SET}

Student feedback can be obtained in a number of ways, for example through questionnaires, informal or formal conversations, quantitative inventories or formal qualitative sessions, or meetings of representative or consultative staff-student committees (Harvey, 2003; Richardson, 2005). The most common method of collecting student feedback is the use of questionnaires. Student feedback questionnaires administered centrally by institutions are regarded as standard student evaluations of teaching which "can provide a broad picture of students' experiences of learning and therefore inform teachers about the effectiveness and quality of both teaching and learning" (Stein et al., 2012, p. 2). Student questionnaires are normally multidimensional. The dimensions may include items indicating: personal attributes, knowledge, and preparation; content of teaching; student-teacher interactions; and teaching aids. These areas for feedback are shown, for example, in the Students' Evaluations of Educational Quality (Marsh, 1982, 1987) and in Student Feedback on Teaching developed by the Centre for Academic Development, Victoria University of Wellington. The SETs from Victoria University includes both Likert scale and openended questions.

As aforementioned, SETs delivered with a particular strategy can be used for formative purposes (i.e., to improve teaching). The following are several features of such formative SETs. First of all, it should provide rich formative information (i.e., students' answers to open-ended questions) for teachers to deepen understanding students' perception of their teaching rather than just ratings from Likert scale questionnaires. Teachers may use SETs to inform changes if they are informative (Penny, 2004), provide new information, encourage the teacher to improve, and facilitate the use of alternative effective methods of teaching (McKeachie, 1979).

In addition, if teachers have their voice in developing SET questionnaires or some autonomy to collect student feedback on targeted teaching aspects (i.e., to focus on their needs), they may make changes to improve practice. Teachers appreciate feedback when they value the data as cues for decision-making and accept that necessary changes may be made even though these may go against their existing values and beliefs (Burden, 2010). If teachers do not have their voice in the evaluation process, the role of SETs may be devalued (Stronge \& Tucker, 1999). When teachers 
design their own ratings form for desired information, rather than use standardised tools, they may gain a better understanding of teaching areas that need improving (Rotem \& Glasman, 1979). Feedback targeted to specific teaching problems may bring about improvement (Marsh \& Roche, 1993). As in Bullock's (2003) study, teachers developed their own SETs by choosing both forced-choice and open-ended items from an existing inventory. This kind of formative SETs may be named teacher-tailored SETs (TT-SETs). Thus, TT-SETs may allow teachers to collect the information they need, bringing them certain autonomy in making decisions on their practice. However, my experience with my TT-SET suggests that through self-designing SET, teachers might miss key areas which need feedback. To overcome this challenge, I believe that this kind of TT-SET may need two sections: a compulsory section including items that give an overview feedback on teaching and an optional section including items that teachers can choose for their needs. Teachers may need to be provided with a repertoire of feedback items from well-established standardised SETs and guides on choosing items for their TT-SETs from it. The implication for this study is that both general standardised items and teachers' self-tailored items would be useful.

Besides the focus on teachers' needs, timing for TT-SET is important for promoting its formative role. The significant impact of the timing of feedback on the improvement of teaching has stimulated debate. Marsh and Roche (1993) drew from their study that end-of-term SETs have stronger effects than those of mid-term feedback. However, they admitted that the generalisability of their conclusion needs further research because of the distortion of irrelevant items such as assignments and examinations in the standardised SET that made the mid-term feedback less appropriate. On the contrary, other researchers such as Penny (2004) and Ramsden (2003) valued earlyterm or mid-term formative SETs because they facilitate teachers' reflection on current practice and can be used as cues for upcoming changes during the rest of the course. Early-term SETs not only make teachers aware of student perspectives but also offer them a chance to reflect on teaching, and recognise the differences between their and students' experience of teaching (Narasimhan, 2001; Penny, 2004). SETs need to be collected earlier in the course (e.g., four to six weeks after the start of the course) to allow for teachers' refinement of teaching aspects that need changing (Hofman \& Kremer, 1983; Seldin, 1989). End-of-term SETs are not useful for the modification of the teaching approach to benefit current students (Belluigi, 2013; Narasimhan, 2001). 
In a study on student perception of SETs, Brown (2008) found that 88.75 percent of student participants believed that mid-term SETs would improve both academics' and students' performance and attitudes towards the class. Ramsden (2003) emphasised: "Evaluation at the end of a course, cannot replace evaluation during it" (p. 225). Earlyterm SETs may be an appropriate means to inform teaching and facilitate teaching improvement.

In summary, there are several features (also conditions) SETs may have so that they can be a lens for teachers' reflection to improve teaching. SETs need to provide teachers with rich information on teaching, focus on teachers' needs (i.e., allow them to choose feedback items for their needs), and should be collected early in the course. This implies that, the so-called TT-SETs need to have open-ended questions in addition to Likert-scaled questions that come from a repertoire of well-established SET items. Also, if collected early in the course, TT-SETs may provide early information on practice so that academics can make timely adjustment to their teaching through reflection. In other words, early-term formative TT-SETs appear to be a promising lens for teachers' reflection and action to improve practice.

\subsubsection{Challenges to the use of formative SET}

\subsubsection{Impact of cultural context}

SETs have been used to evaluate academic quality of teaching in many countries from West to East. For many years, many countries, including the UK, the USA and Australia, have developed instruments for the evaluation of academic quality in tertiary education institutions (Lee, Huang, \& Zhong, 2012). SETs have been of major interest in the tertiary education sector in China (Yin et al., 2014) and have become an emerging phenomenon in Vietnamese tertiary education institutions (Dung \& Mcinnis, 2002). The use of SETs may be influenced by cultural factors in both China and Vietnam, which have Confucian heritage culture where teachers have a special status. In China's case, as $\mathrm{Hu}$ (2002) indicated, traditional Chinese education emphasises the maintenance of a hierarchical but harmonious teacher-student relationships where students are supposed "to respect and not to challenge their teachers" (p. 98).

Both Chinese and Vietnamese teachers share Confucian ideology, suggesting that the use of SETs in Vietnamese tertiary education institutions may face similar cultural 
factors. In Vietnam, the traditional image of academics is depicted as an honourable and respectful identity with high intellectual and moral values and who establish formal and hierarchical relationships with students (Dung \& Mcinnis, 2002). The aforementioned Confucian ideology whereby academics are regarded as noble and their competence is not to be questioned may impact on the academic-student relationship. Academics do not tend to regard students as mature individuals who have independent thoughts, sound perspectives, and ability to evaluate their teachers, or of equal status to their teachers; therefore, they may think that students cannot evaluate them (Dung \& Mcinnis, 2002). If SETs are used for summative purposes, they may make academics vulnerable to criticism (Dung \& Mcinnis, 2002), which may result in their resistance. Students giving feedback on teaching may challenge this formal value perceived by academics. For example, Hao (2013) found that academics "feel a bit harmed regarding student feedback results" although they welcomed SETs for improving practice (p. 104). Academics' privileged noble status may represent a cultural obstacle to the implementation of SETs because of teachers' perception of their status to students and of SET reliability. This study uses TT-SETs for formative purpose. However, investigation into the use of TT-SETs in the Vietnamese context is needed to determine whether it is impacted by the cultural issues outlined.

\subsubsection{Academics' perception of student feedback}

Although reliability and validity are not the focus of this study, the debates over the reliability and validity of SETs may challenge academics' willingness to take SETs seriously and act on student feedback. Academics' perceptions of student feedback or beliefs about its reliability and validity affect how they use SETs for teaching improvement (Stein et al., 2012). There have been many debates about these aspects. Although many studies have claimed that SETs are reliable and valid (Barth, 2008; Moore, 2006; Murray, 1997; Wachtel, 1998), other studies have indicated that students may be biased or distorted in their feedback responses by many factors. For example, as grading leniency and class size influenced students' responses, the same teacher received more positive evaluations when assigning good grades or teaching smaller classes (Greenwald \& Gillmore, 1997; McPherson \& Kennedy, 2006; Moore, 2006). Teachers' appearance can also bias students' responses: attractive teachers received higher student evaluations than their less attractive colleagues (Riniolo, Johnson, Sherman, \& Misso, 2006). Another issue making students' evaluation questionable 
(Burden, 2010) is that students are not skilled assessors due to their lack of experience, knowledge and perspective (Arthur, 2009; Douglas \& Douglas, 2006; Dung \& Mcinnis, 2002; Richardson, 2005; Rotem \& Glasman, 1979; Wachtel, 1998). With such doubt of SET reliability and validity, academics may dismiss student feedback as a source of information for their reflection.

Other academics found problems lay in tension between their view about teaching and that of students (Yao \& Grady, 2005); thus, the academics would have thought that student feedback was distorted. As Yao and Grady exemplified:

As one participant pointed out, students may not necessarily know what was best for them. Even though the feedback truly reflected their thinking of teaching, it could be flawed or misinformed. (p. 123)

If perceived in this way, academics may make limited use of SET for their reflection. For example, the findings from Stein et al. (2012) and Yao and Grady (2005) that academics who were suspicious of SET did not use it or used it in a limited way.

Academics may question the necessity and usefulness of SETs. Not every academic is convinced of their desirability and utility (Spooren \& Mortelmans, 2006). In studies such as Spencer and Flyr, 1992 and Beran, Violato, Kline, and Frideres, 2005), many academics did not use SETs to improve teaching although they regarded SETs as useful. This may raise a possibility of using SETs in conjunction with a supportive mechanism. Yao and Grady (2005) found that academics' use of SETs for teaching improvement was impacted by external factors such as support from administrators, colleagues, and the organisation, and by internal factors such as academics' tenure status and teaching experience, their perceptions of teaching and student feedback, and their general attitude toward the teaching profession. In addition, as Belluigi (2013) cited from Rhodes University's guides for evaluation of teaching, student feedback an angle from which the academic is judging his/her teaching practice - should be triangulated with other viewpoints of teaching from sources such as peer feedback, research, theory, and self-evaluation. These examples suggest that there are a variety of factors influencing academics' use SETs, so supportive mechanisms may be needed, and that if used formatively, SETs may need to be complemented with other sources. 


\subsubsection{SET as a complementary source used with consultation}

SETs are more helpful if teachers discuss them with a consultant or a peer (McKeachie, 1997). There is ample evidence that SETs used in conjunction with forms of consultation can be effective for teaching improvement (Brinko, 1993; Dresel \& Rindermann, 2011; Lang \& Kersting, 2007; Marsh \& Roche, 1993; Marsh \& Roche, 1997; Penny \& Coe, 2004; Rindermann et al., 2007). It is suggested that negative feedback without consultative support and training appears insufficient to encourage improvement (McKeachie, 1997) because teachers may not know how to make changes (Narasimhan, 2001) or may make little attempt to use feedback information (Richardson, 2005). Mere SETs without consultation which offers opportunities for reflection on teaching and examples on how to improve it do not bring about notable improvement (Rindermann, Kohler, \& Meisenberg, 2007). It implies that a form of consultation may promote reflection and provide new teaching ideas.

Peer observation of teaching (POT) appears to be a relevant form of peer support to complement SETs. It is suggested that the consultative process may involve different augmented activities such as workshops, seminars, and class observation, videorecording, and so on (Penny \& Coe, 2004). Classroom observation is an important part in the process because it identifies teaching behaviours as being debriefed in the consultation session (Penny \& Coe, 2004). Teachers' use of formative SETs operated in a complex system whereby support from colleagues is among external variables that influence teaching improvement (Yao \& Grady, 2005). Colleagues can provide a critical lens for teachers' reflection through collaboration and collegial support (Bolton, 2010; Boud \& Walker, 1998; Colton \& Sparks-Langer, 1993; Convery, 1998; Pollard, 2005). An honest self-evaluation with associated peers is one of the typical characteristics of an effective promoter of reflection (Boud \& Walker, 1998). Peer observation has been recommended as a form of collaboration and collegial support for teachers' reflection (Cosh, 1998). In addition, problems relating to student feedback can be moderated through the support of a trusted and thoughtful colleague who conducts observation and shares his/her perspective (Moore, Walsh, \& Risquez, 2007). Academics' reflection and reflective practice can be nurtured in collegial environments where trusting relationships facilitate reflective dialogues and promote thoughtful practice (Bolton, 2010; Colton \& Sparks-Langer, 1993). 
Also, colleagues may offer necessary confrontation and appreciation though collaboration which is supportive of their reflection. Convery (1998) argued that for reflection to occur, it is necessary for teachers to "believe they have the support of others who will sufficiently respect the integrity of their enquiry to enable awkward and uncomfortable self-revelations to be identified" (p. 203). This means the collaboration with peers can provide the sensitive balance between challenge and support. Moreover, academics can be well-informed with colleagues' perspectives because there are substantive aspects of teaching which can be only judged by them (Hutchings, 1996). This means that academics can triangulate information on their practice from both students and peers to appropriately inform future practice. Thus, peer observation of teaching as part of consultation appears to be relevant when coupled with SETs for academics to improve teaching.

The use of formative SETs augmented with POT aligns with the assumption that examining practice from a variety of viewpoints can facilitate effective reflection and subsequent changes. As argued, feedback gathered from many sources and from the self can be effective because it can stimulate "cognitive dissonance created by discrepancies between feedback recipients' self-ratings and feedback sources' assessments" (Brinko, 1993, p. 577). This can lead to changes in behaviour (Brinko, 1993; McLeod, 2014). In summary, in order for academics to engage in reflection and reflective practice, the use of formative student feedback should be augmented with opportunities for consultation and discussion that can be promoted through peer observation of teaching.

\subsection{Peer observation of teaching (POT)}

Besides purposes of observation for training and research, teaching observation is a common practice that basically serves as an evaluative process for personnel decisions or as a formative process for professional development. The evaluative teaching observation might cause teachers negative feelings such as stress, nervousness, or anxiety which might affect their practice negatively because the observer is a supervisor or some authority (Day, 2013). In contrast, teaching observation for professional development is implemented by trusted colleagues or peers (Day, 2013; Malderez, 2003). There are several models of POT, but a model that focuses on teachers' reflection is relevant for personal growth and development (Day, 2013). 
Thus, with an aim to improve teaching, a reflective component is a key for the success of POT. As Cosh (1999) emphasised, "In a reflective context, peer observation is not carried out in order to judge the teaching of others, but to encourage self-reflection and self-awareness about our own teaching" (p. 25). Through sharing teaching perspectives with colleagues, teachers can develop their pedagogical reasoning (Malderez, 2003).

\subsubsection{Definition and purposes of POT}

In order to examine a relevant POT model for this study, it is necessary to examine its purposes, alternative models, and the meaning of POT used in this study. POT has been used for two distinct purposes: "as a developmental means of enhancing the quality of teaching and as a means of evaluating the quality of teaching" (Kemp \& Gosling, 2012). In other words, in addition to its use for an administrative need for achieving standard-based professional teaching, POT is employed to improve the quality of teaching (Hatzipanagos \& Lygo-Baker, 2006b).

POT can be implemented in three distinct models: the 'management/evaluation model', the 'development model,' and the 'peer review model/collaborative model' (Gosling, 2002, 2014). First of all, the 'evaluation' model, associated with a judgmental nature and linked to teachers' appraisal, serves a primarily administrative purpose and involves senior administrative or academic staff who observe other staff for monitoring teaching quality to meet institutional standards and promote best practice. This model benefits the institution. POT for professional development can employ either the development model or the peer review model. These two models are non-judgemental and formative in nature. The 'development' model involves educational developers/experts observing practitioners (Atkinson \& Bolt, 2010; Hatzipanagos \& Lygo-Baker, 2006a), or expert teachers observing others in the department (i.e., a more experienced colleague working with a less experienced teacher to develop their practice) and benefits the observed (Bell \& Cooper, 2011). POT of this type is similar to peer coaching or peer mentoring (Gosling, 2009). Lastly, the peer review/collaborative model involves academic peers observing each other's teaching (i.e., reciprocal teaching observations). It aims to engage teachers in discussion about teaching and self and mutual reflection in order to improve practice. This model offers mutual benefits between peers. It is closest in meaning to the definition of POT described by Bell (2005) or Bennett and Barp (2008) as follow. 
Bell (2005) defined POT as:

...collaborative, developmental activity in which professionals offer mutual support by observing each other teach; explaining and discussing what was observed; sharing ideas about teaching; gathering student feedback on teaching effectiveness; reflecting on understandings, feelings, actions and feedback and trying out new ideas. (p. 3)

This is a collaborative activity carried out by peer colleagues for mutual benefits of both the observer and the observed in learning about teaching (Schuck, 2011). POT also refers to reciprocal teaching observations that facilitate reflection and discussion to gain insight into the learning and teaching process and environment, with a longterm aim of improving students' learning (Bennett \& Barp, 2008). Martin and Double (1998) listed the detailed aims of a peer observation experience as follows:

- To extend and enhance an understanding of personal approaches to curriculum delivery;

- To develop and refine curriculum planning skills in collaboration with a colleague;

- To enhance and extend teaching techniques and styles of presentation through collaborative practice;

- To engage in and refine interpersonal skills through the exchange of insights relating to the review of a specific teaching performance;

- To identify areas of subject understanding and teaching activity which have particular merit or are in need of further development;

- To develop personal skills of evaluation and self-appraisal. (p. 162)

It should be noted that POT is regarded as a strategy for peer review of teaching, which is a comprehensive system of teaching review that involves gaining insight into teaching from a teacher's course or curriculum design, research into classroom practice, teaching materials, and teaching philosophy statement (Chism, 2007; Iqbal, 2013; McMahon, Barrett, \& O’Neill, 2007). POT has become more a process for professional development than a mechanism for performance management (Peel, 2005). This distinction is important for elaborating the meaning of POT for this study.

POT in this study will be used as a developmental means because it can provide academics with a colleague's lens for their reflection in order to improve practice. As 
Cairns, Bissell, and Bovill (2013) stated, "Its underlying rationale is to encourage professional development in teaching and learning through critical reflection, by both the observer and the observed" (p. 573). Therefore, to serve the purpose of this study it is necessary to make explicit the meaning of POT used in this thesis: it denotes a collaborative process whereby academics participate in reciprocal observations of their peer's teaching for purposes of examining teaching practice and learning in the classroom context, and where observations and teaching dialogue lead to reflection with the aim of improving practice.

\subsubsection{Structured POT procedure for reflection}

The consecutive steps in POT can be a systematic way for reflection. Many researchers such as Cosh (1998, 1999), Hammersley-Fletcher and Orsmond (2004), and Bell, Mladenovic, and Segara (2010) have been advocates of POT as part of a reflective approach to improve teaching. Reflection is an essential part of the POT cycle (Bell, 2001). Conscious and systematic reflection which involves both reflecting on their teaching experiences and setting goals and planning for changes (Richards, 2011). Although there have been variations in the use of POT, the POT process for reflection and learning generally includes a pre-observation meeting, a classroom observation, and a post-observation meeting (Martin \& Double, 1998; Pressick-Kilborn \& te Riele, 2008).

\section{Pre-observation meeting}

The pre-observation meeting allows the observed to inform the observer about the specific nature of the event (e.g., background, content, and purpose of the specific lesson, learning intentions, and teaching strategies) and issues about which the observed teacher particularly invites feedback (Martin \& Double, 1998; PressickKilborn \& te Riele, 2008). This means pre-observation planning allows teachers to target aspects for feedback. Other issues are also discussed at this stage such as how teaching will be documented, ownership, confidentiality, and the expressing of opinions (Martin \& Double, 1998).

\section{The observation}

The observation might occur in such a way that it does not affect the observed teacher's usual level of performance, for collaborative developmental experience without 
criticism (Martin \& Double, 1998; Pressick-Kilborn \& te Riele, 2008). A systematic approach to taking notes focuses on issues raised (Pressick-Kilborn \& te Riele, 2008).

This step may also provide vicarious experience because teachers can obtain new teaching ideas from observing their peer (Bell \& Mladenovic, 2008; Finn, Chiappa, Puig, \& Hunt, 2011; Hendry, Bell, \& Thomson, 2013; Hendry \& Oliver, 2012). This aspect POT is also supported by Bandura's (1977b) social learning theory. According to Bandura (1977b), one can learn from others through modelling, observation, and imitation. One's learning is ignited through observing others' behaviours and their outcomes. Bandura (1977b) stated:

Learning would be exceedingly laborious, not to mention hazardous, if people had to rely solely on the effects of their own actions to inform them what to do. Fortunately, most human behaviour is learned observationally through modelling: from observing others one forms an idea of how new behaviours are performed, and on later occasions this coded information serves as a guide for action. (p. 22)

The concept of observational learning in the broader social cognitive theory by Bandura (1977a, 1986, 1997) can be used to interpret the impact of POT on academics' learning about teaching. Observational learning about teaching through POT may facilitate reflection on practice, which then may trigger changes to practice.

\section{The post-observation meeting}

The feedback meeting/post-observation discussion might occur immediately after the lesson (Martin \& Double, 1998; Pressick-Kilborn \& te Riele, 2008). According to Martin and Double (1998), this debriefing requires the observer to be well prepared with their honest and constructive feedback. The participants remind themselves of the targeted learning of the observed session and collaborative reflection to see which aspects of the teaching went well and which ones were less successful. Then, reflective questions may be asked by the observer without imposing their own interpretation of the event, to facilitate discussion on the necessary steps for improvement. Debriefing facilitates the sharing of tacit knowledge which is made explicit through observation (Atkinson \& Bolt, 2010; Carroll \& O’Loughlin, 2014). This step appears to facilitate purposeful critical constructive dialogues. 
An alternative practice may include a fourth stage whereby the observer may write about the observed's strength and aspects that need improving, and the observed may write his/her summary record of the observation, including main points of the teaching dialogue, and responses to the observer's record, and developmental plan for action (Fullerton, 2003). This step may contribute to academics' systematic reflection.

POT can support teachers through this process, facilitating interactions between teachers in the pre-and-post observation meetings (Hammersley-Fletcher \& Orsmond, 2005), with discussion on targeted areas of teaching (Hatzipanagos \& Lygo-Baker, 2006b), high-quality feedback (Fullerton, 1993; Hammersley-Fletcher \& Orsmond, 2004), and reflection (Hammersley-Fletcher \& Orsmond, 2005).

The procedure described above appears relevant to meet the aim of the collaborative POT defined and targeted in this study. In general, the POT process includes preobserving, while-observing, and post-observing activities. These steps are themselves structured and together form a systematic procedure of POT. It is suggested that the collegial support, together with the non-judgmental atmosphere created through the structured POT process, may stimulate teachers' reflection on practice.

\subsubsection{Effectiveness of formative POT}

As presented in section 2.6.1, the 'developmental' and 'collaborative' models of POT can be used for formative purposes and may enhance reflection for teaching improvement. Although many studies face the problem of generalisability of POT effectiveness, its benefits have been clearly documented and are discussed in the following sections. POT has been seen as a process that enhances academics' reflection and reflective practice, professional learning about teaching, changes and aspirations, confidence and self-efficacy, and collegiality.

\subsubsection{Enhancing reflection and learning}

The collaborative POT has been viewed as a process that promotes reflection and provides insights into practice. These insights may contribute to the professional learning of both the observer and the observed.

\section{Enhancing reflection}

Purposeful observation and interaction with colleagues can lead teachers to self-reflect more frequently and explicitly. Though there are variations in the application of 
collaborative POT to improve teaching practice, POT has been regarded as a process that promotes academics' reflection and reflective practice (Barnard et al., 2011; Cairns et al., 2013; Cosh, 1999; Hammersley-Fletcher \& Orsmond, 2005; Peel, 2005). This is achieved by getting them to join in a structured cycle of pre-observation meeting, observing, and post-observation meeting (Pressick-Kilborn \& te Riele, 2008). For example, the thought of getting their practice professionally observed makes teachers think about their current practice and if it could be improved (Cairns et al., 2013). The debriefing session can facilitate academics' sharing of tacit knowledge made explicit though observation (Atkinson \& Bolt, 2010). In addition, POT can provide formative information on practice, such as "fresh orientation on teaching, on student reaction and engagement, on subject delivery and on all sorts of subtle dimensions of teaching that teachers might be otherwise unaware of" (Moore et al., 2007, p. 17). In addition to promoting teachers' awareness of their practice, POT assists teachers to develop their ability for observation and critical reflection on the dynamics and changing setting of learning (Peel, 2005).

A number of examples on the use of collaborative POT can be found such as PressickKilborn \& te Riele, 2008; Bell and Mladenovic, 2008; Cairns et al., 2013; Barnard et al., 2011; and Donnelly, 2007. Pressick-Kilborn and te Riele (2008) carried out collaborative POT at the University of Technology, Sydney, Australia to develop their teaching practice. They observed one lesson each semester over two years. Their collaborative journal and discussion were documented through observation notes and emails which were used in a three-stage process involving pre-observation planning, the observation itself, and post-observation discussion. They reported that active engagement in reciprocal POT provided prompts for articulation of reasoning and ongoing reflection.

Bell and Mladenovic (2008) used collaborative POT as a strategy for a tutor development programme in conjunction with an academic developer at the Faculty of Economics and Business at the University of Sydney to help tutors improve practice. The programme included three development sessions per semester with POT and a self-reflective exercise. Tutors completed a peer observation proforma which was based on student feedback. The rationale to use this form was to encourage them to think about what students expect and could be potentially used to compare the peer feedback with student feedback. Other data came from the development session, an 
end-of-session survey, an end-of-semester survey, and a focus group. The findings indicate that tutors obtained new teaching ideas by watching their peer and reflecting on their own teaching, and were willing to change, or became aware of it.

Another example on collaborative POT was carried by Cairns et al. (2013) to examine a POT scheme for tutors within the Department of Paediatric Dentistry at Glasgow Dental School, the United Kingdom. Random pairing form dyads of peers. The tutors were randomly paired with a peer, who then observed and gave constructive feedback on their teaching. POT engaged tutors in reflection and development of their teaching practice through observations and scholarly discussion. These examples illustrate that POT can be effective for promoting academics' reflection.

POT may stimulate academics to address the question of what (content reflection) and how (process reflection), and why (premise reflection). POT may provoke academics' critical thinking about what aspects of their practice should be promoted and/or changed. In addition to content reflection, POT can facilitate reflection on the process. It allows teachers to discuss and seek valuable feedback on teaching methods and techniques with trusted colleagues (Carroll \& O’Loughlin, 2014).

Premise reflection can be enhanced through POT. For example, Donnelly's (2007) study documents a peer partnership programme that was designed to encourage teachers' critical reflection and enhance their deeper understanding of teaching and learning within a supportive professional environment. This POT programme was carried out at Dublin Institute of Technology, in the Republic of Ireland to explore participants' perception of the impact of collaborative POT scheme offered to develop academic staffs' and faculty members' competencies. Data were collected from evaluation forms, interviews, and documents such as teaching portfolios and pre- and post-observation meeting notes. Academics over a five-year programme were seen to engage in purposeful critical reflection on classroom practice. Findings from Donnelly's study indicated instances where participants compared their teaching quality against experiences and knowledge of relevant educational theory. Participants went further than the how and addressed the why of teaching, as evidenced in one participant's quote: "in writing reflectively about the experience you were subconsciously trying to figure out why you would do this and whether you were doing it better" (Donnelly, 2007, p. 124). 


\section{Personal learning}

Reflection promoted through POT can bring about new understandings which contribute to academics' professional learning. POT can be regarded as a selfdevelopment opportunity and a learning experience for both observer and observed; it provides teachers with new insights into their own teaching (Carroll \& O'Loughlin, 2014; Pressick-Kilborn \& te Riele, 2008; Sullivan et al., 2012). Learning that occurs in POT is often attributed to the observed teacher after he/she receives the observer's feedback and engages in reflection (Cosh, 1999; Gosling, 2002; Hammersley-Fletcher \& Orsmond, 2004; Yiend, Weller, \& Kinchin, 2014). However, the observer can learn as well by adopting the observed peer's practices (Bell \& Mladenovic, 2008; Hendry \& Oliver, 2012) or thinking about solutions for problems emerging in the peer's classroom and referring to their own context (Tenenberg, 2016). Teachers can learn new teaching practices and develop motivation and confidence to use these strategies in their practice (Hendry et al., 2013; Hendry \& Oliver, 2012). For example, PressickKilborn and te Riele (2008) reported that POT brought them fresh insights into pedagogy, curriculum, their students, and themselves as teachers and learners, which contributed to their professional learning. Participants in the study by Hendry et al. (2013) observed their colleagues' teaching situation by watching students' reaction and level of engagement, made judgments on which strategies worked and were worth trying, and found out new strategies for promoting student interactions to enhance their engagement with curriculum material. These examples appear to illustrate single-loop learning described by Argyris and Schön (1974). Moreover, as previously mentioned, participants in Donnelly's (2007) study engaged in premise reflection, which led them to deeper learning or transformative learning. This is an instance of 'double-loop' learning (Argyris \& Schön, 1974) or 'theory building' (Korthagen \& Lagerwerf, 2001). Reflection stimulated through POT may lead to academics' new comprehensions that contribute to their learning.

In summary, POT has been used for a developmental purpose and has been regarded as a process that encourages academics' reflection on practice and even reflective ability. It is a structured process and provides formative information that may stimulate the articulation of reasoning and reflection on content, process, and premise. These outcomes may result in academics' new comprehensions that contribute to academics' 
professional learning. The peer collaboration with respect and trust may contribute to academics' willingness to learn from a peer.

\subsubsection{Increasing perceived changes in current practice and aspirations for future changes}

Engaging in POT will likely lead to academics' changes to current practice. PressickKilborn and te Riele (2008) engaging in their own collaborative POTs assert that these observations have provided a catalyst for change by giving them fresh understanding of their practices. POT has the capacity to bring a positive change to teachers' teaching practice, helping them refine teaching skills or try new ideas (A. Bell \& Mladenovic, 2008, 2015; M. Bell \& Cooper, 2013; Hammersley-Fletcher \& Orsmond, 2005; Hendry \& Oliver, 2012; Potter et al., 2011; Shortland, 2010). For example, Bell and Mladenovic (2008) reported changes tutors made after observing each other's tutorials and giving feedback: "The most commonly reported change was encouraging more student interaction (eight tutors). Other changes included reflecting more on their teaching, being more enthusiastic and using visual aids" (p. 745). In reference to Bell's (2001) categorisation of changes (i.e., "technical", "pedagogical" and "critical" (p. 33)), Donnelly (2007) also found that his participants made technical changes (e.g., the use of audio-visual media in the classroom) and more profound pedagogical changes (e.g., developing students' critical thinking, communication, and collaboration skills; strategies for motivating students in class; and content sequence cohesion). For instance, one participant said: "I received a lot of very valuable feedback on how I delivered my lectures, particular on my use of video and audio clips, which I was able to put into use straight away" (Donnelly, 2007, p. 126). This piece of evidence implies that the academic engaged in process reflection and learnt how to solve the teaching problem. This also means that the academic learnt from the peer's suggestion and incorporated it into his/her own practice. Critical changes, such as adopting reflective practice as a strategy for ongoing professional improvement, were also perceived (Bell, 2001). Peel (2005) stated: "my experience of POT heightened my alertness, and stimulated my sensory perceptions of my own physical presence in the classroom, and the human value of the teacher as the principal 'teaching aid"” (p. 495). POT can further lead to a transformative change in academics' educational perspectives (Bell, 2005). 
However, not all learning can immediately be transformed into practice. Arguably, POT is not a one-off activity and may not bring about immediate change because development is an ongoing process of observing, reflecting, and learning (Donnelly, 2007; Engin \& Priest, 2014; Shortland, 2004, 2010). Changes may be small but accrued over time through POT. Observing colleagues teach and questioning their own assumptions about teaching make "a mindful teacher" (p. 7) as stated by a participant in Engin and Priest's (2014) study.

Engaging in POT will also likely stimulate academics' aspiration for future changes. Pressick-Kilborn and te Riele (2008) through reciprocal POT reported that "we also highlighted some of the broader changes that we intended to make as a result of our post-observation conversations" (p. 72). Bell and Mladenovic (2008) reported that 28 tutors $(88 \%)$ said that they would change their teaching as a result of POT. They were not sure whether their findings represented significant, lasting transformation of tutors' educational perspectives but indicated their willingness to change or to start thinking about it. These findings mean POT can promote willingness and readiness to change (Wlodarsky, 2005), or awareness of it (Bell \& Mladenovic, 2008). These examples suggest that as a result of POT teachers may start setting aspirations for changes to future practice.

\subsubsection{Enhancing confidence and self-efficacy}

Another outcome that POT could bring is enhanced confidence and self-efficacy. First, POT can help build academics' confidence (Barnard et al., 2011; Bell \& Cooper, 2013; Cairns et al., 2013; Carroll \& O’Loughlin, 2014; Donnelly, 2007; Pressick-Kilborn \& te Riele, 2008). That teachers are encouraged to share understandings of practice and offer each other support through POT can enhance their affirmation of practice and aspiration for further exploration of their practice (Donnelly, 2007; Harper \& Nicolson, 2013). For example, Donnelly's participant said: “it was honestly great to be observed and to observe others; it gave me assurance that I can teach", and another said: "I felt more confident that I had been working along the right lines" (p. 125). It is argued that if academics feel competent and find their changes meaningful, they will sustain what they do (Paulsen \& Feldman, 1995). Thus, the development of confidence appears a positive outcome of POT which may be meaningful to teachers' practice. 
Further, research findings showed that the reciprocal POT had positive impacts on academics' self-efficacy, beliefs about their capacity to use a new strategy (Donnelly, 2007; Hendry \& Oliver, 2012). For example, a participant in Hendry's and Oliver's study thought that "it also gives you good ideas ... you start thinking 'I could do this" (p. 4). This result is most likely because POT appears to trigger three among four types of experiences that may contribute to the enhancement of self-efficacy: vicarious experiences, mastery experiences, and verbal persuasion (Bandura, 1977a, 1997).

Through vicarious experience, observers' self-efficacy is sometimes enhanced. This means seeing a colleague use teaching strategies successfully helps enhance teachers' self-efficacy (Hendry \& Oliver, 2012), as a participant said: "it gave me a particular impetus to try it, having seen it working" (p. 4). Watching a peer teach can also lead to teachers' affirmation of current teaching practice, reassurance that their teaching is similar to that of their peer (Harper \& Nicolson, 2013; Hendry \& Oliver, 2012) because "their colleague did things they already did" (Hendry \& Oliver, 2012, p. 5). In this observational learning, the academics could observe teaching approaches being used successfully and perceived they could also perform them successfully.

In addition, mastery experience through POT may also contribute to the enhancement of self-efficacy. Applying successfully new strategies they learn from their colleague can promote teachers' self-efficacy (Hendry et al., 2013; Hendry \& Oliver, 2012). For example, Hendry's and Oliver's participant said: “And I found that later on I started doing that [i.e., using examples] in my classes and the students reacted positively towards that... they were like, "Yeah, that makes sense now"' (p. 4). It suggests that observing the peer's successful performance stimulated the teacher to try new strategies, and when he/she yielded similar success, it may have led to the enhancement of the teacher's belief in what he/she is capable of.

Verbal persuasion in professional conversations may enhance self-efficacy. Some academics learnt new strategies from the peer observer's suggestions in the postobservation discussion (Donnelly, 2007; Harper \& Nicolson, 2013; Hendry \& Oliver, 2012). Hendry's and Oliver's participant commented that 'the feedback they received was useful because it was affirming - 'you know you're doing this very well'"' (p. 6). It suggests that engaging in teaching dialogue may have offered the academic valuable feedback on teaching strategies and enhanced the person's belief in his/her teaching capacity. 
In summary, POT may enhance academics' confidence and provide possible sources of self-efficacy. The development of this sense of professional worth seems important for changes in academics' practice because a sense of self-efficacy is believed to be associated with intrinsic motivation to act.

\subsubsection{Enhancing collaboration and collegiality}

Although collegiality is often associated with being cooperative, pleasant, and supportive, Freedman (2009) suggests that a more precise definition of collegiality would include "shared power and authority among colleagues" and "cooperative interaction among colleagues" (p. 378). For example, enhanced collegiality may include willingness to act as a support person or teaching model and sharing teaching materials among colleague academics. When POT is conducted under supportive conditions, it is believed to lead to the development of collegiality (Bell, 2005; Bell \& Cooper, 2013; Byrne, Brown, \& Challen, 2010; Donnelly, 2007). The developing collegiality and sense of tight network across the school were perceived from "the building of relationships and camaraderie" (Bell \& Cooper, 2013, p. 67). With the opportunity for mutual engagement in constructive teaching dialogue, spending much time together helps develop good working relationships, mutual understanding, and a sense of belonging to a community (Byrne et al., 2010; Harper \& Nicolson, 2013; Long \& Hoa, 2010; Schuck, Aubusson, \& Buchanan, 2008). The development of high trust relationships deepened collegiality (Shortland, 2010). The development of collegiality appears important to the success of POT because it can help further develop professional relationships. The development of collegiality and professional relationships is a crucial element of POT (Donnelly, 2007). This may be seen as a twoway effect: the nature of POT is collaborative, but its implementation may lead to strengthened relationships between peers, resulting in enhanced collegiality. This development can increase teachers' willingness to collaborate with their peer to further explore their practice through POT. Harper and Nicolson (2013) found that through POT, academics appreciated how they integrated into the flexible community of practice through the building of new friendships and developing understanding of their ability to self-develop. Thus, the enhancement of collegiality seems to be both a benefit and a facilitator of POT. 


\subsubsection{Conditions for POT to be effective}

Three conditions for workplaces to encourage tertiary teachers' professional learning are: spaces for creation of shared meaning; power relationships to encourage collegiality and participation; and relevant procedures for stimulating reflection (Knight, 2006). The literature review suggests POT has potential to create contextual learning that promotes a collaborative teaching environment (Bell, 2001; Long \& Hoa, 2010; Potter et al., 2011; Shortland, 2010) and appears to provide these conditions when implemented under the principles of parity and reciprocity.

\section{Creating an environment for shared meaning}

One of the key features of collaborative POT is to promote reciprocal learning which results from the opportunity for peers to observe teaching and talk to each other. Pressick-Kilborn and te Riele (2008) used a reciprocal POT to develop their practice and found that it supported their learning about their own practice by offering suggestions for change and mutual reassurance. Brookfield (1995) recommends that POT must be reciprocal. Gosling (2014) in discussion on collaborative peer-supported review of teaching argued that its potential for professional learning is maximised if peers "accept the space created as one for mutual or reciprocal learning" (p. 23). Reciprocity acknowledges shared learning in that "seeing you allows me to see myself differently and to explore the variables we both use" (p. 83). These examples suggest that in POT both peers are equally open to learning. This principle of reciprocity is a crucial difference between POT for professional development and POT for evaluation (Pressick-Kilborn \& te Riele, 2008). This kind of shared meaning from both peers may also require to be offered confidentially (Gosling, 2014) because there are instances of academics being concerned about the confidentiality of POT (Bell \& Mladenovic, 2008; Hammersley-Fletcher \& Orsmond, 2005).

\section{Mediating power relationships to encourage collegiality}

Teachers' learning can be nurtured in a POT process that creates a non-judgemental, collegial, and supportive environment. Learning can be achieved when both peers are open to accept challenge from each other and their communication is not distorted by power imbalance which hinders open dialogue (Gosling, 2014). This ideas indicates the importance of the peer relationship (Carroll \& O'Loughlin, 2014) which needs to be collegial and open to facilitate open conversation and honest reflection (Barnard et 
al., 2011; Carroll \& O’Loughlin, 2014; Fullerton, 2003; Hammersley-Fletcher \& Orsmond, 2005; Pressick-Kilborn \& te Riele, 2008; Shortland, 2010). Relationships of openness, trust, and support characterise a collaborative culture (Hargreaves \& Dawe, 1990). Thus, if collaborative POT is a form of collaborative peer review it must include parity at least in the process (Byrne et al., 2010; Gosling, 2014; Schuck et al., 2008). In reality sometimes judgments are made based on assumed greater knowledge and experience. Yet, judgmental feedback was viewed as ineffective (Barnard et al., 2011; Hammersley-Fletcher \& Orsmond, 2005). Both peers having equal roles in POT (as peers asking questions to help each other reflect on practice (Barnard et al., 2011; Gosling, 2014; McGill \& Beaty, 1995)) would promote collegiality, an important condition for peer review (Ambler, Chavan, Clarke, \& Matthews, 2014). A nonthreatening and supportive atmosphere is important to teachers' engagement in reflection and learning.

\section{Following appropriate procedure for reflection}

POT offers academics a structured process that allows peers to examine their focused teaching issues in a systematic way (see section 2.6.2). Does POT structured in such a way automatically lead to learning and teaching improvement? The answer may depend on how academics use formative information that POT provides. As aforementioned, vicarious experience that POT provides can be useful to teacher learning. However, Peel (2005), reflecting on her experience with POT, argued witnessing "techniques, teaching aids, or trick of trade" is not sufficient for improving teaching, but it "requires a synthesis of substantive knowledge, a critically reflective engagement with teaching practice, and a confidence in one's self' (p. 495). Among the steps of POT, teaching dialogue in the post-observation meeting is highly valuable in the learning process because it can enable critical reflection (Byrne et al., 2010; Gosling, 2009). Although there is evidence that this follow-up discussion in POT can promote learning (e.g., Pressick-Kilborn \& te Riele, 2008; Donnelly, 2007), it is uncertain that academics talking about the practice will result in new understanding. Palmer (1998) argues that if teachers do not have anything to discuss in depth rather than "tips, tricks, and techniques", these kinds of conversation "fail to touch the heart of the teacher's experience" (p. 11). Haigh (2005) through his own reflection suggests that conversation can be conducive to learning when it includes discussion about emerging ideas and puts these ideas under ongoing critique, encouraging an 
exploration of ways of articulating them. The discussion suggests that to achieve the potential of POT as a process for teachers' reflection and learning, it is necessary to focus on promoting peers' teaching dialogue in addition to the experience of observing a peer's teaching.

\subsubsection{Challenges to peer observation of teaching}

Although the effectiveness of POT for academic professional development has been documented, concerns about the use of POT have been reported.

First of all, there is a concern about the potential discomfort that POT could bring about. This reservation can come from perceived issues such as "implicit judgements being made, and the effect on students or the dynamic of teaching of having observers in the room" (Cosh, 1998, p. 175). This negative impression of teaching observation as judgmental may derive from the fact that it was once used for evaluative purposes (i.e., when teachers hear about teaching observation of any kind, they think of its 'evaluative' purpose first). For instance, English language academics in China have developed a negative impression of classroom observation "because of its subjective, judgmental, and impressionistic nature" (Wang \& Seth, 1998, p. 205). Even though the 'collaborative' model of POT (Gosling's peer review model) was used for academics across disciplines as first-time users of POT in University of Limerick, Ireland, academics' notion of it being a 'judgmental' process still existed prior to completing the POT process (Carroll \& O’Loughlin, 2014).

A sense of discomfort may result from academics' perception that POT may bring about personal vulnerability. Tensions were reported because POT represented an intrusion into academics' professional autonomy (Blackwell \& McLean, 1996; Iqbal, 2013; Lomas \& Nicholls, 2005; Spencer, 2014). There were fears about confidentiality and personal vulnerability if the observers and observed came from the same school (Hammersley-Fletcher \& Orsmond, 2005). Teaching is an individual and private activity, so disclosure of teaching practice to colleagues can be a new experience (Carroll \& O’Loughlin, 2014).

Academics' anxiety might arise because they have limited knowledge of the POT process. Participants in a POT scheme for chair-side tutors at Glasgow University Dental School expressed anxiety before being observed (Cairns et al., 2013). Irish firsttime participants of POT had a feeling of nervousness or fear of the unknown (Carroll 
\& O'Loughlin, 2014) because they did not know what to expect from POT and where to obtain information. Lack of familiarity with the POT process may make academics reluctant to participate.

There are also concerns associated with academics' responses to institutional implementation of POT. As observed by Byrne et al. (2010) in a study on POT for professional development in one department in a university in the South of England, academics perceived that POT lacks value for their development: they regarded it as a yearly 'ticking the box' exercise to be done for institutional purposes. POT used in this way had little impact on academics, resulting in little or no change to teaching practice. This means that POT is viewed as a means of compliance with institutional policies rather than a mechanism to meet academics' real desire to change practice (Shortland, 2004) when its developmental purpose becomes an institutional requirement (Gosling, 2014). Even worse, when POT is used for administrative purposes (e.g., appraisal model), it becomes meaningless to academic development because there is no worthwhile feedback. The process becomes instrospective with mutual backpatting/slapping (Byrne et al., 2010; Cosh, 1998). These examples suggest that when POT is compulsory for institutional purposes, there seems to be greater likelihood that POT will be perfunctorily practised and will not be valuable to academics' learning.

In addition, there is concern about the impact of the nature of the peer relationship on teaching dialogue. Interactions between colleagues may be influenced by social relationships involving power and authority (Gosling, 2002, 2014; HammersleyFletcher \& Orsmond, 2004). There can be anxieties around giving feedback and how colleagues might receive criticism because receiving negative feedback or perceiving feedback as criticism could damage confidence. From a peer observation process from the School of Law and the School of Sciences within one post-1992 university in the UK, findings by Hammersley-Fletcher and Orsmond (2005) indicated a tendency to view critical feedback in a negative way: "Despite knowing each other well, and describing their relationships as open and honest, lecturers nevertheless worried about giving and receiving criticism" (p. 218). Distance in relationship may hinder peers' engagement in rich open dialogue about teaching (Schuck et al., 2008). As a result, the feedback process may face more difficulties because personal relationships make it complicated (Hammersley-Fletcher \& Orsmond, 2005). Peers would have found it hard to give negative feedback to each other (Cairns et al., 2013; Carroll \& 
O’Loughlin, 2014). Also, close relationships between peers also hindered open critical feedback; only positive aspects of teaching were discussed to avoid confrontation and protect their relationship (Carroll \& O’Loughlin, 2014).

Besides possible impact of the peer relationship on teaching dialogue, there is a concern that academics are too ill-equipped to provide effective feedback. Gosling (2014) gave an example that participants in a workshop made widely differing judgments on the teaching they observed through a video. Another issue was that peer observers did not focus feedback on the observed academic's particular needs (Wang \& Seth, 1998). These examples support the idea that training is necessary for academics to provide effective feedback (Cosh, 1998, 1999).

Another challenge lies in academics' availability for the implementation of POT because of limited time and heavy workloads. For example, Adshead, White, and Stephenson (2006) reported that time constraint was a barrier to participation with twothirds of the teachers agreeing that POT would take too much time and paperwork (on questionnaire survey carried out with general practitioner teachers of medical undergraduate students from King's College London School of Medicine at Guy's, King's College and St Thomas' Hospitals). Similarly, one of the problems identified in Donnelly's (2007) POT schemes concerns time management: several participants found that preparation for POT and paperwork were time-consuming. Long and Hoa (2010) in a study on critical friend group using POT in a Vietnamese university found that it was hard to arrange post-observation meeting times to suit all peers. However, when the benefits outweigh the time, academics can still manage to participate. As a participant noted in Byrne et al.'s (2010) study on peer development: “Takes more time but benefit outweighs this. Allows ongoing discussion on issue to be followed up, more structured process - felt less disjointed, got much more from it” (p. 225).

It is obvious that POT has its limitations. However, examining the benefits the POT may offer (see section 2.6.3) suggests that it is worth implementing collaborative POT for more insight into this process. I support Bell's and Cooper's (2013) argument that "where an initiative is evaluated negatively it should not be assumed that peer observation has failed because of the nature of the thing itself" (p. 61). The value of POT should be further considered with attention paid to its formative purpose and structured process that is believed to have contributed to academics' learning. 


\subsubsection{Cultural context of implementation}

Examining the condition for effective POT and its challenges suggests that one of the keys to successful POT is the peer relationship. Different contexts may have different variables that impact on POT, so it can be assumed that relationships between people in different cultures have different characteristics and mores. Thus, it may be necessary to have an overview of cultural variations in relationships and the Vietnamese cultural context of relationships.

\subsubsection{Overview of cultural variations in relationships}

This brief overview of Western and non-Western cultural values acknowledges cultural influences on how people communicate with awareness of the significance of relationship. People in different cultures may have different beliefs and values in the maintenance of harmony in interpersonal relationships. Researchers studying cultural variations in relationships have typically indicated the distinction between individualist and collectivist cultures (Hofstede, 1997; Lalonde, Hynie, Pannu, \& Tatla, 2004; Reis, Collins, \& Berscheid, 2000). Individualism and collectivism are two fundamental values that clearly differentiate between Western cultures and nonWestern cultures (Lalonde et al., 2004; Phinney, Ong, \& Madden, 2000). Individualist cultures focus on the individual's rights and personal goals whereas collectivist cultures emphasise the rights and well-being of the groups of which individuals are the members (Lalonde et al., 2004; Reis et al., 2000) and thus focus on group interdependence, harmony in interpersonal relations, and conformity to group norms (Phinney et al., 2000; Reis et al., 2000). The latter view tends to prevail in Asian cultures (Reis et al., 2000). These cultural values may regulate the way people communicate in their interpersonal relationships. The cultural environment and social interaction have enormous impact on an individual's values, and the guiding principles of their thought and behaviour (Moore \& Asay, 2013). Values refer to stable longlasting beliefs about what is desirable or important to a person, and cultural values are used to explain people's personal behaviour and motives in a culture (Hofstede, 1997). Because POT concerns human relationships, acknowledging these cultural values and beliefs may be useful for exploring how a globalised predominantly Westerndeveloped process works in a different local context like the Vietnamese context. 


\subsubsection{Vietnamese cultural context concerning relationships}

It is important to consider possible contextual impacts before international models for academic professional development are adopted in Vietnam. The discussion on the use of the intervention processes will be lacking if Vietnamese culture is not taken into consideration with respect to human relationships.

Vietnamese regard harmony, shame, and face as important factors that relate to their status with others. Vietnam, as well as other Asian countries such as China, Korea, Singapore, and Japan, has been under the impact of Confucianism, sharing certain qualities of a collectivist society (Mai et al., 2005; Tuyet, 2013). Thus, harmony is viewed as the crucial determinant of a relationship. With much care about self-respect and face saving, direct confrontation (public criticism and humiliation) should be avoided (Mai et al., 2005; Tuong, 2002) because it is considered rude (Hofstede, 1997). Face can be seen as equivalent to honour or prestige and portrays one's proper relationship with his or her social environment, which is important to that person and his or her family (Hofstede, 1997). Losing face means losing prestige, resulting in feeling ashamed. Therefore, feeling ashamed is what Vietnamese never want to experience because shame or a painful feeling of humiliation is not only a personal matter but also the family's or group members' concern. The loss of face brings a bad feeling to people in all cultures, but it appears more damaging to Vietnamese (Borton, 2000). Saving face and avoiding criticism are often viewed as a preferable way of maintaining harmonious interpersonal relationships.

Vietnamese accept the principle of social hierarchy, so they have a tendency to consider their relative status in communication with others. Relationships in Vietnamese society are characterised by the concept of power distance, "the extent to which the less powerful members of institutions and organizations within a country expect and accept that power is distributed unequally" (Hofstede, 1997, p. 28). One of the key principles of Confucian teaching is that the stability of society is based on unequal relationships between people; thus Vietnam is considered a country with large power distance in relationships (Hofstede, 1997). In interpersonal interactions, Vietnamese accept the principle of social order (Ashwill \& Diep, 2005; Borton, 2000; Jamieson, 1993). They tend to care about their relative status by considering their addressee's age, sex, social status, and level of intimacy to establish a correct relationship in verbal communication (Ashwill \& Diep, 2005; Jamieson, 1993) and to 
perform the correct behaviour in accordance with the nature of this relationship; otherwise, they could lose face (Thang, 2013). In other words, people must consider the level of power distance in their relationship to have appropriate behaviour in communication. Showing respect is the underlying premise for such behaviour (Borton, 2000). It is a cultural practice that great deference is paid to those of higher status, regarding their social position, age or organisational position (Thang, 2013; Tuong, 2002). Respect is regarded as the foundation of any relationship in Vietnamese society.

Examples about the perceived impact of this cultural issue can be found in the Vietnamese context or China (with similar culture). Thuy (2012), in a study on POT for beginning English language teachers' professional development at the Faculty of English Language Teacher Education, Hanoi University of Languages \& International Studies, Vietnam National University, found that one of the reasons for participants' trouble finding a peer stemmed from what she described as face-saving attitude from potential participants. This means these teachers were not willing to be observed by a peer for fear of losing face when all aspects of their practice would be put under scrutiny. In the Chinese context, $\mathrm{Hu}$ (2005) argued that teachers tend to give positive comments and avoid giving critical feedback to their colleagues because such criticism is commonly perceived as face-threatening; therefore, the post-observation conversation would become shallow and less challenging than it might be.

In summary, the discussion of the Vietnamese interpersonal relationship suggests that its associated issues such as harmony, shame, and face or respect affect how people communicate under the principle of social order. Because POT is a form of collaboration, it may be impacted by this cultural aspect. Therefore, academics' beliefs and values relating to interpersonal relationships (if any) documented in this study may be considered in the discussion of findings.

\subsection{Summary of the literature review and research gap}

\subsubsection{Summary of findings from the literature on formative SET and POT}

The review of literature addressing areas such as teachers' pedagogical reasoning, reflection and reflective practice, and lenses for reflection in tertiary education 
suggests that reflection is a mechanism for the development of academics' professional knowledge. Shulman's (1987) model of pedagogical reasoning and action particularly focuses on an idea of teaching that emphasises comprehension and reasoning, transformation and reflection. Hence reflection is viewed as an underlying factor of teachers' decisions on what and how to teach in their context and as a way of gaining insights into practice. To engage in effective reflection, it is suggested that teachers use multiple external perspectives (e.g., Brookfield, 1995; Loughran, 2002). The external lenses may be students and colleagues, two of the four important reflective lenses suggested by Brookfield (1995). They appear to be contextual variables (particularly structural aids) to reflection in LaBoskey's (1993) model that discusses how reflection can be triggered.

The discussion on the use of SETs for formative purposes suggests they can be effective for teachers' reflection and learning but need several conditions for them to be effective. First of all, if SETs focus on teachers' needs and reflect their voice, they would have stronger impacts (e.g., Stronge \& Tucker, 1999). This means they need to provide formative information teachers need and they can choose areas for feedback. Besides, it is suggested that SET collected early in the course (or mid-term) may facilitate teaching improvement and benefits the current students (Belluigi, 2013; Keutzer, 1993; Narasimhan, 2001). These requirements support the argument for the use of early-term TT-SET which allows teachers certain autonomy to explore teaching aspects for their need and time to examine practice for improvement. It is also argued that SET effectiveness may depend on teachers' capacity to reflect and learn from SET data (Ramsden, 1991; Winchester \& Winchester, 2011). Thus, there is a need to provide teachers with a supportive mechanism. Particularly, SET may be effective for teaching improvement if it is coupled with a consultation process (Dresel \& Rindermann, 2011; Lang \& Kersting, 2007; Penny \& Coe, 2004; Rindermann et al., 2007) such as workshops, seminars, and class observations. POT may be one among the forms of consultation where teaching behaviours can be debriefed.

POT has been a popular practice, a formative process for academic professional development in many countries. The collaborative POT (Gosling's (2002) so-called 'peer review' model) is carried out by trusted colleagues (Day, 2013; Malderez, 2003) and engages teachers in teaching dialogue and reflection to improve practice. POT is seen to promote teachers' reflection and reflective practice that may enhance their 
learning, changes to practice, confidence and self-efficacy, and collegiality. Besides teaching discussion, POT also emphasises observational learning (Bandura, 1977b) where academics can learn from watching their peers teach. The focus of POT is to trigger academics' reflection in order to improve practice. For POT to be effective, it may require several conditions such as: creating an environment for shared meaning, mediating power relationships to encourage collegiality, and following appropriate procedure for reflection.

However, the use of SET and POT may face several challenges. The implementation of SET may face challenges concerning teachers' perception. Teachers may not use it because of its lack of reliability and validity (e.g., Stein et al., 2012) which is also associated with cultural issues (e.g., Dung \& Mcinnis, 2002; Hao, 2013). Academics in Confucian heritage cultures perceive that SETs are distorted and believe they have an honour status and students are not 'mature' to give profound feedback on practice. Therefore, these perceptions may result in the rejection and limited use of SETs.

The challenges to POT include a sense of discomfort, distortion by the nature of the peer relationship, negative responses to institutional use of POT, lack of skills to give effective feedback, and time constraints and heavy workloads. Among many factors, many studies indicate that the peer relationship is the key to the success of POT (e.g., Carroll \& O'Loughlin, 2014). For example, its nature determines whether open critical dialogue can be facilitated: closeness in the peer relationship can hinder deep critical discussion to avoid confrontation and protect the relationship (Carroll \& O'Loughlin, 2014). Cultural variations in relationships with reference to the Vietnamese Confucian heritage culture suggests that Vietnamese cultural values concerning social order, harmony, face, shame, and so on may influence the way people communicate in interpersonal relationships. This implies an area to explore in the use of POT which requires collaboration between peers.

In summary, the evolving understanding of how SET and POT have been used through research and debate provides an important socio-cultural and historical context for my study. Student and colleague lenses can be used in the forms of TT-SET and POT respectively. TT-SET and POT, supplementing each other, may allow academics certain freedom to obtain targeted important information on their performance in order to improve practice. These sources of information for academics to improve practice 
are emerging issues in Vietnamese tertiary education institutions. This summary relates the findings from the literature to the research gap that informed this thesis.

\subsubsection{The research gap}

In order to enhance insights into practice, it is suggested that academics examine practice from a variety of viewpoints. TT-SET and POT are argued to be the relevant lenses for teachers' reflection and deeper understanding about teaching. These two reflective lenses supplement each other: TT-SET is feedback from direct recipients of the teaching process while POT provides a professional's perspective.

SET or POT has been commonly used for formative purposes in both the West and the East, and has been proved to be useful for teachers' professional learning. Although challenges to SET and POT have been documented in the literature, they may have different challenges in different socio-cultural contexts. This study explores how SET and POT work with academics in a Confucian heritage culture like Vietnam. The TTSET augmented with POT will be implemented without any modifications. In other words, this study will not change the nature of the intervention as it is originally intended in Western research, particularly POT, to fit into the Vietnamese context. Instead, this aspect of cultural impact will be considered in the discussion of findings from the use of the intervention to make recommendations for improved future application. Research is contextually situated, and research in education needs to consider and be responsive to issues of diversity. Thus, it is useful to examine how TT-SET augmented with POT works in the Vietnamese context.

In addition, few studies employ TT-SET augmented with POT in Vietnam. SETs have been used in Vietnamese tertiary education institutions but are underexplored with regard to their use for improving teaching. Professional development in Vietnamese universities has been often based on event-based activities (e.g., workshops, training courses, and conferences), and non-formal activities like POT are under-investigated in Vietnamese tertiary education institutions (Hiep, 2001). Some Vietnamese studies on peer mentoring or critical friend groups that employ POT as a strategy focused on beginning teachers who are believed to be more inclined to learn, so it raises a need to examine how POT works with more experienced academics. In some cases, POT is implemented informally and unsystematically (i.e., self-initiated by some colleagues 
in the same faculty), and this suggests a need to implement POT systematically with a structured procedure.

The research gap this study aims to address is, therefore, how TT-SET augmented with POT impacts on academics' pedagogical reasoning, with a focus on their reflection, in the Vietnamese context. 



\section{Chapter 3. Methodology}

This chapter discusses the methodological approach and the design of the study. First, it presents the research objectives and research questions, which were drawn from the research gap in the literature review. Then, the theoretical framework is depicted and illustrated. This chapter discusses why the selection of the constructivist paradigm and use of a case study design are relevant to the research.

\subsection{The research objectives and questions}

As previously argued, teacher reflection and deeper understanding of teaching and learning through a process of pedagogical reasoning and action can contribute to the enhancement of teaching practice. Thus, it could be productive to employ an intervention process for professional development that promotes teacher reflection and reflective teaching, assisting academics to enhance awareness of practice, and refine and/or transform understanding of teaching and learning. Research on the implementation of TT-SET augmented with POT as formative lenses for academics' reflection and changes to practice in the Vietnamese context may make a useful practical and theoretical contribution to understanding ways for academics to improve practice in this context. The study aims to answer the research question: In the context of a Vietnamese university, how does TT-SET augmented with POT impact on academics' pedagogical reasoning?

There are three sub-questions:

1. What is the perceived impact of TT-SET augmented with POT on academics' practice, beliefs, and attitudes?

2. What are academics' perceptions of the effectiveness of TT-SET augmented with POT for promoting their reflection in the process of pedagogical reasoning?

3. What are possible challenges to the implementation of TT-SET augmented with POT in Vietnam's context?

\subsection{The theoretical framework of the study}

As discussed in Chapter 2, reflection plays an important role in teachers' intentional learning because it is a mechanism for enhancing understanding. For this study a merged framework was developed on the basis of my review of the literature. This 
framework draws on teachers' reflection for understanding their pedagogical reasoning (particularly changes in their practice with a focus on reflection, learning, and action) and underlying factors affecting these changes. The framework was adapted from Shulman's (1987) model of pedagogical reasoning and action and LaBoskey's (1993) reflective model. These models assume that there is connection between teachers' reflection and their learning (new comprehension) which may lead to their subsequent action for improved practice.

Shulman (1987) viewed teaching "as comprehension and reasoning, as transformation and reflection" (p. 13), so his model was intended to explain how teachers decide what and how to teach within their teaching context. In other words, the model illustrates reflective practice during the teaching process (i.e., Shulman emphasises teaching as reflective practice). An act of teaching may start with an understanding of content knowledge and purpose which is transferred into teachable forms. After delivering instruction, a teacher can evaluate student learning and his/her teaching by looking back on original objectives, at what was achieved and how it was achieved, and adjust for experiences. This act of evaluation can be promoted into reflection whereby the teacher critically examines practice and uses evidence he/she could gather to support explanations, allowing him/her to analyse the teaching experiences, promote what is successful, make changes to what needs improving, and build upon knowledge. This means reflection may lead to new understanding of teaching, students, and the subject, which then may guide subsequent action. Hence reflection may underlie teachers' decisions on what and how to teach in their own context. Reflection is considered as a mechanism that contributes to professional learning (e.g., Argyris \& Schon, 1974; Lagerwerf, 2001; McAlpine \& Weston, 2000; Harrison, 2008). Reflection is one of the important elements in Shulman's (1987) model of pedagogical reasoning and action. It is assumed that, in the process of pedagogical reasoning, reflecting on actual teaching performance (i.e., instruction) may contribute to teachers' learning. However, Shulman did not identify or discuss the means by which reflection may be encouraged and supported.

In a model developed for use with preservice teachers, LaBoskey (1993) argues that reflection can be triggered by both internal impetus (e.g., puzzles of practice, selfassessment, etc.) and external impetus (e.g., collegial interaction, professional requirements, etc.). She identified that there are contextual variables such as location, 
timing, and structural aids that influence reflection. The results of reflection may be a new comprehension. LaBoskey also clarifies the nature of a new comprehension, which could be an improved reflective ability, an added or changed belief about pedagogical content knowledge (e.g., curriculum, subject matter, or instruction), an attitude or value about the premise of teaching (i.e., theoretical assumption) and necessary for the reflection process, or a change in emotional states. New comprehensions may or may not result in action in current and future practice.

At any rate, the new comprehensions should always be tentative and subject to continual revision... Reflective teachers reflect in order to learn to improve their understandings of, feelings about, and responses to the world of teaching. These new comprehensions may or may not actually serve to incite or inform solutions to current or future practical problems. Thus the solving of practical problems is seen to be a secondary outcome of reflection. (LaBoskey, 1993, p. 35-36)

LaBoskey develops this framework for reflection with respect to a complex interaction of cognitive abilities, beliefs, values and attitudes, and emotions in a problem-solving context on the basis of a review of literature and empirical research. By drawing out internal and external variables influencing reflection in teacher education, LaBoskey's model can be a means of considering how reflective practice can be promoted. Additionally, the content of reflection can be impacted by the conditions of reflection (LaBoskey, 1993). This 'context' from LaBoskey's model can be complementary to Shulman's 'reflection'. To encourage reflection, it is necessary to create a context for reflection. This context can be provided by structural aids to reflection such as research-like tasks, collegial support (partners/observers), seminar discussions, and so on (LaBoskey, 1993). According to LaBoskey, structural aids can influence the nature and quality of the outcomes of reflection. The structural aids appear to be an important variable that can encourage reflection and new comprehension. As LaBoskey (2004) argues, "Since knowledge is experiential and knowledge generation is critical reflection or inquiry as stance, teacher knowledge can best be understood, transformed, constructed, and articulated by the teacher self in collaboration with others" (p. 826). Many researchers (e.g., Loughran, 2002; Bolton, 2010; Harrison, 2008; and Convery, 1998) have argued that reflection can be facilitated through opening practice to scrutiny by some form of collaboration with others. These arguments imply that structural aids such as peer collaboration can be applicable to inservice university teachers as well. 
Shulman's model and LaBoskey's model can be associated with each other in a way that the former emphasises teachers' reflection in the process of pedagogical reasoning while the latter focuses on the mechanism with which reflection can be enhanced. Shulman's model explains how reflection may lead to new knowledge and possible action while LaBoskey's model explains how reflection can be promoted. This is the rationale for a merged framework to examine the use of TT-SET augmented with POT in promoting academics' reflection and their perceptions of its impact on their thinking and practice. TT-SET and POT can be viewed as either structural aids to reflection or external impetus for reflection. In other words, for reflection on practice, academics can use TT-SET and POT as complementary sources of feedback on their observable teaching acts (instruction). Students and colleagues fit into the framework as lenses for reflection.

These two contextual elements for reflection (i.e., TT-SET and POT) were added to the merged framework in light of Brookfield's (1995) lenses for teachers' reflection. Brookfield (1995) suggests that critical reflection can be facilitated by multiple lenses, including autobiographies as teachers and learners, students' eyes, colleagues' experiences, and theoretical literature. However, for reflection to be a genuine mirror to see practice, it is important for one to see one's practice through others' eyes (Loughran, 2002), (i.e., opening one's practice to scrutiny by others (Bolton, 2010)). Confrontation and collaboration may be needed for effective reflection (Convery, 1998). As discussed in the literature review, students and colleagues are the two important lenses for teachers to critically examine their teaching. Students are the direct recipients of the teaching process, so their feedback provides ultimate information on teaching that teachers need to assess and address students' concerns and expectations to make changes for improvement. However, there are many substantial aspects of teaching that can only be judged by other teachers (Hutchings, 1996). Both sources of information (from students and colleagues) on teaching complement each other: the former is the view of the recipient of the teaching process while the latter is the view of a professional. This means, in the process of pedagogical reasoning, a teacher's reflection can be supported with viewpoints from students and a colleague that give feedback on his/her teaching. Through collecting student feedback on their teaching through TT-SET, identifying their teaching aspects to be observed and improved, conducting reciprocal POT, a teacher can critically examine 
his/her teaching, beliefs and assumptions about teaching, and explore new possibilities for professional action. In addition to new comprehension about educational topics, according to LaBoskey, the outcome of reflection can be an understanding of the reflection process itself (e.g., reflective abilities, values and attitudes for reflection). As a result, the conceptual framework guiding this study (illustrated in Figure 3.1) was developed on the basis of Shulman's (1987) model of pedagogical reasoning and action and LaBoskey's (1993) reflection model.

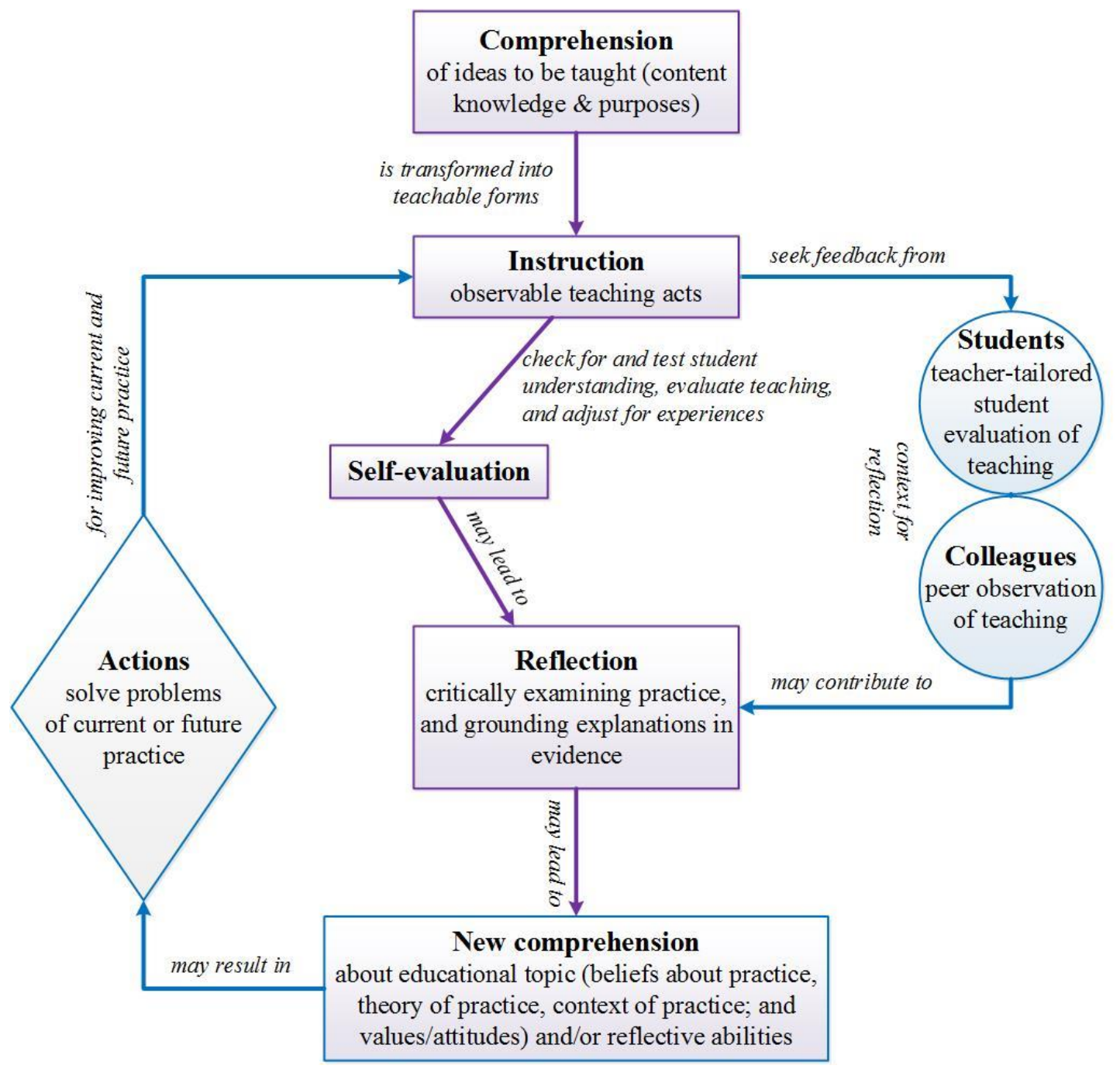

Figure 3.1: The theoretical framework for the study (developed from Shulman's (1987) pedagogical reasoning and action and LaBoskey's (1993) reflection model)

SETs have been used in tertiary education institutions in many countries, including Vietnam (e.g., Lee, Huang, \& Zhong, 2012; Yin, Lu, \& Wang, 2014, Dung \& Mcinnis, 2002; Hao, 2013). They can serve either summative or formative purposes. Researchers such as Rotem and Glasman (1979), suggest that if teachers design their 
own student feedback form to collect information they need, they may become more focused, be more aware of, and gain better understanding of their practice. This resonates with my personal experience which is presented later on to illustrate the theoretical framework. Thus, TT-SET, as an informal form of student feedback, may be useful in promoting teachers' reflection.

To enhance the effectiveness of student feedback, it is suggested that student feedback be used in conjunction with consultative support (McKeachie, 1997; Rindermann et al., 2007). Among other forms of consultation, classroom observation is an important part (Penny \& Coe, 2004). The literature has indicated that colleagues can provide a critical lens for reflection through collaboration and collegial support (e.g., Boud \& Walker, 1998; Colton \& Sparks-Langer, 1993; Covery, 1998; Pollard, 2005). POT is seen as a collaborative developmental activity in which colleagues provide mutual support by observing each other (Bennett \& Barp, 2008). It is a means to improve teaching (Kemp \& Gosling, 2012). In addition, the use of POT is supported by Bandura's (1977b, 1997) social learning theory in which observational learning is a focus. As argued in the literature review, a most-commonly used theoretical framework for the POT schemes is the reflective practice model (Bell, 2001). Thus, the assumption is that TT-SET augmented with POT can be useful for academics' reflection on practice. In other words, the theoretical framework focusing on using students and colleagues as reflective lenses situates TT-SETs plus POT as a strategy within the broader spectrum of evidence-based feedback on teaching.

Reflection with recourse to external resources such as student feedback and peer observation of teaching may lead to radical changes in practice because, as Bandura (1986) indicated, people are the agents of change in both themselves and their environment through their interaction with that environment. It is assumed that TTSET augmented with POT can promote reflection on practice (immediate outcome). Then, reflection may lead to new comprehensions (intermediate outcome). These new understandings may result in a new possibility for action taken to improve current or future practice (ultimate outcome). Because this study looks at academics' pedagogical reasoning under the intervention - a possible means to promote reflection, becoming aware of reflective abilities can also be a possible outcome together with changes in values and attitudes. Although emotional states are important in reflective teacher education as LaBoskey claimed, this aspect is not the focus of the study. Thus, for the 
purposes of this study, I assume that new comprehensions can be about educational topics (e.g., pedagogical content knowledge) and/or reflection itself. In addition, new understandings may or may not lead to action to solve current or future practical problems, so solving problems is regarded as a secondary outcome of reflection (LaBoskey, 1993). In other words, the theoretical framework supports this cycle: by reflecting on teaching - which is the result of the process of transforming content knowledge into teachable forms - through external reflective lenses, academics may gain new comprehensions, which may or may not trigger actions to improve current and future practice.

To illustrate the merged model augmented with the addition of student and colleague lenses, I will draw on my professional experience as a teacher of English for business. The first time I taught students how to describe a graph (i.e., I started with comprehension of content and purpose), I used a sample paragraph to explain the structure of a paragraph of graph description. I explained sentence structures and popular words for describing trends, asking students to write sentences based on prompts, and then asked them to describe a particular graph (i.e., these were ways knowledge was transformed into teachable forms and presented in the classroom). By checking student writing, I realised that the teaching techniques I used were not really effective in developing students' writing skill (i.e., evaluation - looking at my original objectives and reviewing what was accomplished and how it was accomplished). Thus, I began asking for their feedback on my teaching with self-designed SET which also included open-ended questions (i.e., looking for student lens). I then realised that students needed more practice to memorise verbs for describing trends, and required different structures to describe trends (i.e., reflection and new comprehension). I planned to use integrated skills (i.e., use listening, speaking, reading, and writing skills) in teaching writing (i.e., prepared for action). For the following session in a different class, I started with a listening task when teaching how to describe a graph. Students then got some slight idea of the structure of a paragraph of describing trend, several verbs, and structures. Then, I gave them a similar graph and asked them to work in pairs describing the graph using the words and structure to which they had just listened. After that, they were asked to read a sample paragraph and identify the structure. They also completed a task of sentence building and sentence transformation before writing a complete paragraph. 
For the similar next session in another class, I invited a colleague to observe my teaching and give feedback (i.e., looking for colleague lens). One suggestion was that I should create an engaging atmosphere in the classroom. I then used the peer's feedback to refine teaching by asking students to join in a role-play activity whereby students played the role of a reporter describing a trend. This really added excitement to the classroom, and students could easily complete the writing task later on. My own experience illustrates that reflection is at the core of changes in my pedagogical reasoning. At first, I reflected on teaching by collecting evidence of student learning. However, then I thought I should reflect from a variety of viewpoints to see if the changes I made were effective in promoting students' learning, so I took into account student and peer lenses and found them useful for guiding changes.

The theoretical framework does not include other elements of LaBoskey's framework such as steps of the reflection process, the necessary attitudes, or what content they should be aware of because the study is not intended to train academics with these elements. The merged framework does not focus on the steps of the reflection process itself. This part in LaBoskey's model was left out because this study focuses on changes in academics' pedagogical reasoning when TT-SET augmented with POT was implemented. So were the attitudes for reflection and the content of reflection. However, these two aspects may be referred to if they emerge from the data during analysis.

Although the use of TT-SET augmented with POT may raise challenges (see sections 2.5.4 and 2.6.5), it appears to be a prospective process for a formative purpose. A few studies on POT in Vietnam have been conducted on beginner teachers and have not identified the conditions for it to be effective. In addition, the use of standard SETs and random supervisory teaching observation by leaders in the university where I teach seems not beneficial to improving academics' teaching. These academics are not provided with information they need and constructive feedback from observers through a structured process. Besides, supervisory observations seem to bring about the observer effect, resulting in perfunctory modification of certain teaching aspects. Hence, my literature review and personal experience leads me to investigate whether TT-SET augmented with POT is beneficial to academics' learning. My assumption is that TT-SET and collaborative POT focus on academics' needs to solve teaching problems, so they may enhance academics' reflection on teaching which may lead to 
their new understanding and subsequent action. This assumption raises several questions: Is the intervention process worth implementing at the Vietnamese institution? What modification may be necessary for the intervention to be effective? What further research needs to be done for the implementation? These questions inspire me to establish the purpose of the study.

This research aims to explore the extent to which TT-SET augmented with POT contributes to changes in academics' pedagogical reasoning. This study examines academics' perception of the intervention process and how it may contribute to their reflection on teaching. Thus, it is interpretive in nature, describing the particularity of the case and suggesting explanations for the outcome by linking to the context. The study is also exploratory because it may develop ideas for further research. It aims to investigate the extent to which TT-SET and POT works in the Vietnamese context. Particularly, this study examines the adoption of POT into the Vietnamese cultural context with a model close to those in the literature from Western settings, exploring if there are any cultural issues which may pose potential challenges to the implementation of POT.

\subsection{Research paradigm, methodological approach, and method}

\subsubsection{Selection of approaches}

In order to choose an appropriate method for the study, it is necessary to select a relevant research paradigm. A paradigm is a way of perceiving the world with underlying assumptions that shape thinking and action (Mertens, 2005). Guba and Lincoln (1994) identified three questions that help define a paradigm:

1. The ontological question: "What is the form and nature of reality and, therefore, what is there that can be known about it?"

2. The epistemological question: "What is the nature of the relationship between the knower or would-be knower and what can be known?"

3. The methodological question: "How can the inquirer (would-be knower) go about finding out whatever he or she believes can be known?" (p. 108)

According to Mertens (2005), there are four paradigms for research: positivist/post positivist, constructivist, transformative, and pragmatic. They are different in beliefs 
about the nature of reality (ontology), the nature of knowledge and the relation between the knower and the would-be known (epistemology), and approach to systematic inquiry (methodology). This study examines whether or not the intervention process can lead to changes in academics' pedagogical reasoning; it is not intended to look for whether it makes an impact on practice (i.e., not intended to measure improvement), so it is not designed with predetermined variables of interest. Therefore, the positivist paradigm is not appropriate. This study looks for more nuanced effects and insights into how teachers perceived TT-SET augmented with POT. Considering the paradigms and the research context, the constructivist paradigm is appropriate for conducting this study. It assumes "a relativist ontology..., a subjectivist epistemology..., and a naturalistic... set of methodological procedures" (Denzin \& Lincoln, 2011, p. 13).

I aim to understand the meaning or knowledge constructed by these academics, the way they made sense of their teaching world and their experience with this world. In other words, my epistemological stance is closely associated with the constructivist paradigm. Researchers adopting the constructivist paradigm acknowledge that "knowledge is socially constructed" (Mertens, 2005, p. 12). The study focuses on the reality constructed by its participants; thus, multiple interpretations of the reality are expected (Merriam, 1998; Mertens, 2005; Stake, 2010). Therefore, this research looks at different interpretations of the mechanisms of TT-SET augmented with POT by English language academics and their perceptions of the intervention process (i.e., examining meanings participants constructed as they engaged with the context they were interpreting) (Merriam, 1998). The interpretation of the intervention was also impacted by my perspective as a researcher (e.g., through interpreting videorecordings of academics' performances or their responses in interviews). This is because in a qualitative study the researcher plays the role of interpreter and data gatherer, his perspective and bias may influence the interpretation of the findings (see section 3.5).

The qualitative methodology and inductive logic approach were appropriate for categorising meanings emerging from participants' perceptions and different interpretations of TT-SET augmented with POT for teaching improvement in Vietnamese tertiary education. Qualitative research methods such as interviews, peer observations, video-recordings and document analysis (POT protocols) are interpretive in nature. Although there was quantitative analysis of student feedback 
(i.e., calculating the means of early-term TT-SET items and comparing the means of these items with those in the end-of-term TT-SET), the study was mostly qualitative. A qualitative study was appropriate for this research because it helps to achieve four of the five intellectual goals of qualitative research that Maxwell (2005, pp. 22-23) describes:

- To understand "the meaning, for participants in the study, of the events, situations, experiences, and actions they are involved with or engage in" and "how their understanding influences their behaviour."

- To gain insight into "the particular context within which the participants act, and the influence that this context has on their actions"

- To "identify unanticipated phenomena and influences, and generating new, 'grounded' theories..."

- To identify "the process by which events and actions take place"

These four goals support the choice of a qualitative methodology for the research questions. It helps the researcher understand the meaning of TT-SET augmented with POT for reflection constructed by participants within particular contextual conditions. It may also help to identify unanticipated events and understanding of the intervention process. In addition, an inductive approach was appropriate for exploring how English language academics interpreted and responded to the mechanisms of TT-SET augmented with POT and how they behaved during the intervention process. The multiple data sources, analysed for patterns and themes, allowed the researcher to develop concepts and understandings of the practice of TT-SET and POT for professional development in the context of the selected university.

In summary, the description of the paradigm and the research context show that the constructivist paradigm best suited the study based on the discussion of reflection and reflective practice in the literature. While my prior experience and the initial literature review on the use of SET and POT indicated some possible outcomes, the study was conducted to gain deeper understanding of the relationship between TT-SET augmented with POT and academics' pedagogical reasoning and action, and to gain insights into the participants' perspectives and their perceptions of the outcomes of the intervention process as well as possible challenges to its implementation in Vietnamese higher education. 


\subsubsection{Case study design}

\subsubsection{Features of case study design}

A case study is defined as "a method for learning about a complex instance, based on a comprehensive understanding of that instance obtained by extensive description and analysis of that instance taken as a whole and in its context" (U.S. General Accounting Office, 1990, p. 15). The case study can be further described by its special features: particularistic, descriptive, heuristic, and inductive (Merriam, 1988).

- Particularistic: the study focuses on a particular phenomenon which is studied within its real-life context (Merriam, 1988; Yin, 2009, 2011).

- Descriptive: "the end product of a case study is a rich, 'thick' description of the phenomenon" (Merriam, 1988, p. 11). As Stake (2010) explains, "a description is rich if it provides abundant, interconnected details, and possible cultural complexity, but it becomes thick description if it offers direct connection to cultural theory and scientific knowledge" (p. 49).

- Heuristic: the case study helps the reader gain insights into the phenomenon and discover new meaning, extend experience, and confirm previously unknown factors (Merriam, 1988; Yin, 2011).

- Inductive: the case study allows the researcher to discover new relationships, concepts, and understanding rather than verify hypotheses (Merriam, 1988). In analysing qualitative data, according to Thomas (2006), "inductive analysis refers to approaches that primarily use detailed readings of raw data to derive concepts, themes, or a model through interpretations made from the raw data by an evaluator or researcher" (p. 238). The inductive approach facilitates social constructivist data interpretation that leads to "a complexity of views" (Creswell, 2009, p. 8)

The definition of a case study and its features implies that it is an appropriate research design to examine a phenomenon systematically (Merriam, 1988). The following section provides the justification of the choice of case study as a research design for the current study. 


\subsubsection{Relevance of case study design for the research}

Methodological choice depends of what is desired to be known (Merriam, 2001). The decision to choose a non-experimental research design such as a case study depends on whether it aims to answer the question 'how' or 'why' to examine a contemporary phenomenon over which the research has little or no control (Merriam, 1988; Yin, 2009). Another deciding factor for the selection of a case study is if a bounded system can be identified as the focus of the research (Merriam, 1988). As an emerging researcher, I was exploring how academics perceived the implementation of TT-SET augmented with POT (over one semester) and the ways in which it might influence their changes in pedagogical reasoning and action with a focus on their reflection. A qualitative case study can be appropriate for examining the intervention for the following reasons.

My choice of a case study first of all depended on the nature of the research question, amount of control, the desired end product, and the boundedness. This case examination of the intervention in a Vietnamese university aims to explore how it impacts on academics' pedagogical reasoning. Additionally, the use of TT-SET augmented with POT is a contemporary event involving complex social situations with a multitude of variables which cannot be controlled (Yin, 2009). This is why an experimental or quasi-experimental design was not appropriate. Furthermore, the intervention can be regarded as a bounded system that allowed the researcher comprehensive understanding of its complexity because, unlike other types of qualitative research, case studies are "intensive descriptions and analyses of a single unit" (Merriam, 2001, p. 19).

In addition, this study has the typical features of a qualitative case study which are aforementioned. First, it is particularistic because it focused on a particular phenomenon - the intervention of TT-SET augmented with POT to promote academics' changes in pedagogical reasoning. Second, it is descriptive because it described interconnected details of the phenomenon and possible cultural issues associated with the intervention. The description came from multiple data sources (Yin, 2011) such as student feedback, teachers' reflection reports, video-recordings, and observer feedback report and debriefing. The case study can accommodate varied perspectives of the participants (Merriam, 2001). The sources available included multiple perspectives of the academics from observing and being observed. Third, it 
is heuristic because it allowed in-depth understanding of TT-SET augmented with POT with descriptions from various sources of data in a real-world setting with respect to participants' perspectives, context, and emerging issues associated with the intervention. Finally, it relied on inductive reasoning because it discovered new understanding from the implementation of the intervention rather than verification of pre-determined hypotheses. It helped gain understanding of the existing or emerging meanings from participants' perception and interpretation of the intervention from a diversity of evidence sources.

In summary, a qualitative case study design was appropriate for the research question to be explored. It was used to understand a real-life phenomenon in depth, and such understanding holds within important contextual conditions, namely examination of TT-SET augmented with POT that encompassed the context of a Vietnamese university. The intent of the study is to gather sufficient information about the research issue to analyse, interpret, and theorise about the phenomenon (Merriam, 2001). This case examination of the intervention is an interpretive case study which contains descriptive data "used to develop conceptual categories or to illustrate, support, or challenge theoretical assumptions held prior to the data gathering" (Merriam, 2001, p. 38). These theoretical assumptions derived from the literature review are presented in the form of propositions which were used to guide data collection, as discussed in the next section.

\subsubsection{Necessity of propositions for the research}

As Yin (2009) states: "each proposition directs attention to something that should be examined within the scope of the study" (p. 28). While case study approaches are related to the development of theory rather than the verification of hypotheses, it is natural that researchers have some assumptions of possible outcomes at the outset of a study. My review of the literature, my prior experience with TT-SET and POT, and my experience as a teacher contributed to my prior assumption that TT-SET augmented with POT may be a useful process for teachers' reflection and professional learning about teaching to improve teaching practice. This assumption is actually a proposition. It is an orientation that guides the data collection process (Yin, 2009). Using propositions in a constructivist case study for data analysis could seem to potentially limit thorough understanding of the case's complexities and emerging findings because they may restrict the data analysis. However, the use of these prior 
assumptions was made at the research design stage to orient the study with key findings and assumptions that emerged from the literature review. Using these propositions as a starting point was to help ensure the collection of sufficient data within the scope of the study.

In designing this qualitative case study, I made choices of methodology which were instrumental to the inquiry. I used Yin's idea of propositions and Merriam's guidance in a case study design. The following are reasons for my choice: Yin (2009) proposes a carefully detailed design. He suggests that in order to avoid internal and external inconsistency, a case study must be designed with the following components: "the study's questions; its propositions, if any; its unit(s) of analysis; the logic linking the data to the propositions, and the criteria for interpreting the findings" (p. 27). These components at the design stage help direct attention to what should be studied within the scope of the study and the methods to be used. However, Yin's philosophical stance is inclined towards positivism (Yazan, 2015) because Yin suggests linking data to propositions in analysis which looks like verifying hypotheses and appears to restrict emerging findings. Stake's (1995) view of case study is a more flexible design that allows major modification when carrying out the research. According to Stake (1995), case study researchers need to develop a set of two or three issue questions (research questions) that will "help structure the observation, interviews, and document review" (p. 20). Merriam (2001) gives clear guidelines and advice to identify the theoretical framework from the review of the relevant literature that will guide the research. Merriam discusses detailed steps in designing qualitative research, including reviewing relevant literature, developing a theoretical framework, identifying a research problem, sharpening the research questions, and selecting a sample for data collection (purposive sampling). I followed these steps; yet, I also found Yin's use of propositions was helpful to my research because the theoretical propositions (derived from the literature review) reflected important theoretical issues and directed me "where to look for relevant evidence" (Yin, 2009, p. 28). Theoretical propositions helped make sure that data essential to answer the research questions were not missed. In other words, the use of theoretical propositions derived from the literature review helped sharpen the data collection tools for the focus of the study and ensure sufficient data were collected. For example, they helped focus the questions to be asked during 
the interview with participants. Thus, the propositions underlying the research questions governed the decisions about data sources and collection methods.

\subsubsection{Challenges of the design}

One of the challenges of a case study is that its results may be limited to describing the phenomenon (Merriam, 1988). TT-SET augmented with POT described in this thesis was the first study of the implementation of TT-SET coupled with a systematic voluntary reciprocal POT process in the selected university. There were only 11 participants forming five pairs and a group of three (i.e., one academic was paired with two peers). Because POT was not already embedded in the culture of the university and there were no incentives to encourage academics' participation, the researcher believed it could be difficult to access a large number of participants. However, its strength may outweigh its limitations. A case study "offers a mean of investigating complex social units consisting multiple variables of potential importance in understanding the phenomenon" (Merriam, 1988, p. 32). The study involved multiple data collection methods, through which data are triangulated for reasonable interpretation of the phenomenon. Triangulation is a means of providing a rich description of the phenomenon.

The second challenge is that because it is desirable to provide rich, thick description of the phenomenon, I believed that the data collection process should be more than one semester. However, I was unable to have more than one semester to collect data for the study. This is because of the reality of the research context: academics were overwhelmed with workload and it was impossible to ask them to join in a long process. One semester of participation in the research was reasonable to them. That is why the study was conducted in only one semester. This is one of the limitations of the study. So, this study was an initial investigation to indicate if the implementation of the intervention process is appropriate in the university context. It can be used as a basis for further research into the area.

The next concern is that the quality of the case study depends on the sensitivity and integrity of the researcher because he/she is the instrument of data collection and analysis and his/her bias can affect the interpretations of findings (Merriam, 1988). The study is interpretive and depends on the researcher's interpretation. Therefore, personal bias needs to be made explicit. In section 3.5, I present my self-examination 
of assumptions and personal bias to prepare for the research, including information on my background and reasons for doing the work.

The final concern is that the use of propositions to focus data collection may give readers the impression that it contradicts the inductive approach of analysis. Thus, the rationale for them is made explicit in section 3.3.2.3. In addition, the danger of using propositions may affect the nature of the study if the researcher uses them during the data analysis stage. I separated the self from the propositions stated in the early stage of data collection by using an inductive approach to data analysis.

\subsection{Design of the study: case study design}

Research designs are considered as "logical" plans, not "logistics" plans, with the meaning that they guarantee that the data collected and the strategies for analysing them address the intended research questions (Yin, 2011). There are no fixed designs in qualitative research, so researchers can make various choices to customise a design that best fits their study. A single case study (a programme case study with embedded cases) (Stake, 2006) was chosen to capture factors influencing the implementation of TT-SET and POT as well as teachers' perceptions of its impact and the changes they displayed. In order to guide and direct my thinking and action relating to the scope of the study and methods of data collection and analysis in the design process, I referred to the five components that Yin (2009) suggested a case study research design should have: the study's questions, its propositions, its units of analysis, the logic linking data to the propositions, and the criteria for interpreting the findings. This section describes the logic of the design by presenting the case selection and the units of analysis, and the links between the initial theoretical propositions in relation to the research questions, and the sources of data.

\subsubsection{Case selection}

\subsubsection{The case}

Stake (1995) depicts the case as "a specific, a complex, functioning thing" (p. 2). Merriam (2001) sees "the case as a thing, an entity, a unit around which there are boundaries" (p. 27). The case can be a person, a programme, a group, a specific policy and so on. According to Merriam a qualitative case study is "an intensive, holistic description and analysis of a bounded phenomenon such as a program, an institution, 
a person, a process, or a social unit" (p. viii). Merriam's perspective aligns with Miles' and Huberman's (1994) definition of the case "as a phenomenon of some sort occurring in a bounded context" (p. 25). When a phenomenon of interest can be specified and its boundaries (of what will be inquired) can be drawn, then it can be a case. Thus, the case for this study is the implementation of TT-SET augmented with POT in a Vietnamese university. Exploring how the intervention impacts on academics' pedagogical reasoning also means examining what changes occur and what mechanism leads to the changes. Also, the participants in this study teaching in the same context selected their own peer for POT, so they are embedded units within this single case (Baxter \& Jack, 2008).

\subsubsection{Units of analysis}

There has been confusion in the literature about the term units of analysis. "The unit of analysis, or 'the case', can be an individual, a program, an institution, a group, an event, a concept" (Merriam, 1988, p. 44), which implies that the case and the studied phenomenon are the same. The determinant of the case is "what it is you want to be able to say something about at the end of the study" (Patton, 2002, p. 229), and Patton advocated that "cases are units of analysis" (p. 447). Yin (2009) also considers units of analysis as cases. However, he states that units of analysis can be defined one way though the studied phenomenon is actually defined in a different way. This definition implies that units of analysis and cases are different. Yin suggests that units of analysis will emerge when the primary research questions are specified. The above definitions create confusion in distinguishing the unit of analysis from the case, and are not useful to my study. I found Grünbaum's (2007) description more useful. Grünbaum (2007) disambiguates the unit of analysis and asserts that "the unit of analysis is identical with the knowledge that key informants can provide the researcher with" (p. 88).Therefore, the "case" in this study is the TT-SET augmented with POT - the process/programme, while the unit of analysis is the perception (the knowledge) that the academics of Business English (the key informants) had on the implementation of the intervention, regarding their pedagogical reasoning (with a focus on reflection on practice). This differentiation aligns with the definition of the case study, a single case study with embedded cases. This intervention process was investigated in a Vietnamese tertiary education institution. 


\subsubsection{Sample selection}

Qualitative research usually involves "small samples of people, nested in their context and studied in-depth" (Miles \& Huberman, 1994, p. 27). Purposive sampling was used to select "the most productive sample to answer the research question" (Marshall, 1996, p. 523). The type of sampling used in this study was what Merriam (2001) calls nonprobability sampling, often employed in qualitative research. The most common form of this type is purposive or purposeful sampling (Patton, 1990). I decided that the use of this sampling method can help me learn the most from the sample (Merriam, 2001) and select information-rich (embedded) cases which can be studied in depth (Patton, 1990). I selected the academics of business English in one department because this homogeneous sample (Patton, 2002) enabled me to describe "the experiences of subgroups of people who share similar characteristics" (Mertens, 2005, p. 318). This choice is called convenience sampling (Patton, 2002) because these participants were accessible to me in terms of my experience and understanding of the academics and the research context.

In order to choose the sample I wanted, I first determined a list of criteria which were essential to the study (Merriam, 2001). These were academics who: (1) had both teaching and research responsibility, (2) taught business English, (3) had at least three years' teaching experience, (4) had a masters' degree, (5) taught in the same department. The participants will be described in section 4.2.

These criteria reflect the purpose of the study. Criteria 1,2, 4, and 5 reflect the assumption that colleagues who share similar characteristics can be supportive of each other in POT. Another reason is that the academics could easily work together because they know each other and come from the same department. When they teach the same subject, it is easy for them to understand the nature and focus of teaching in order to give useful feedback. It was the first time POT was carried out in the university. If I approached academics from different disciplines and departments, I may not have found participants easily. Criterion 3 shows the intent to explore the perceived impact of POT on more experienced academics, which as a group have not previously been formally studied in Vietnam. The review of the literature on POT in Vietnamese tertiary institutions suggests that previous studies have focused on beginner academics. 


\subsubsection{Propositions in accordance with the research questions}

This section outlines the propositions following the research questions.

Main question: In the context of a Vietnamese university, how does TT-SET augmented with POT impact on academics' pedagogical reasoning?

Proposition 1: The process of getting students' and peers' feedback with classroom visits and debriefing sessions will enable academics to make their personal theory of practice explicit. This assumption derives from the formative uses of SET (e.g., Ramsden, 2003; Winchester \& Winchester, 2011) and POT (e.g., Barnard et al., 2011; Peel, 2005; Atkinson \& Bolt, 2010) (see sections 2.5.2 and 2.6.3.1).

Proposition 2: Feedback from students and peers will promote learning about teaching, especially pedagogical content knowledge, because it will facilitate reflection and reflective practice. This assumption derives from possible effects of SET and POT on teachers' learning (e.g., Belluigi, 2013, Donnelly, 2007; Shortland, 2010, 2004; Hendry, Bell, \& Thomson, 2013) (see sections 2.5.2 and 2.6.3.1).

Proposition 3: Academics will use formative feedback from their students and peer to inform their practice or guide and direct changes to their practice for teaching improvement. This assumption derives from possible effects of feedback from students and colleagues in academics' action for current and future practice (e.g., Brookfield, A. Bell \& Mladenovic, 2008; M. Bell \& Cooper, 2011, Bell, 2001) (see sections 2.4.2 and 2.6.3.2).

There are three sub-questions:

Sub-question 1: What is the perceived impact of TT-SET augmented with POT on academics' practice, beliefs, and attitudes?

Proposition 4: If academics target the areas of their teaching for consideration, they will be intrinsically motivated to refine their practice (single-loop learning) and rethink and restructure their assumptions about teaching (double-loop learning). It is assumed from the argument in section 2.5.3 that when teachers decide the aspects of their teaching to be considered, they may have a sense of autonomy which may contribute to their motivation to take action (see section 2.3.2). Teachers' learning is assumed to fall into single-loop or double-loop learning (Argyris \& Schon, 1974). 
Proposition 5: If the TT-SET augmented with POT brings positive effects, the academics will be aware of their beliefs and attitudes about their capacity. This assumption derives from the reasoning of possible effects of TT-SET (see section 2.5.3) and POT (e.g., increasing confidence or self-efficacy (Barnard et al., 2011; Bell \& Cooper, 2011; Hendry \& Oliver, 2012)) (see section 2.6.3.3).

Sub-question 2: What are academics' perceptions of the effectiveness of TT-SET augmented with POT for promoting their reflection in the process of pedagogical reasoning?

Proposition 6: The process will help academics promote self-evaluation and integration of teaching theory and practice. This proposition derives from reports suggesting that teachers through POT (e.g., discussing and seeking valuable feedback on teaching with peers) may examine their current practice and a possibility for improvement (e.g., Cairns et al., 2013; Carroll \& O’Loughlin, 2014) (see section 2.6.3.1).

Sub-question 3: What are possible challenges to the implementation of TT-SET augmented with POT in Vietnam's context?

Proposition 7: Teachers' perception of student feedback validity and reliability may hinder their use for teaching improvement. This is assumed from reports suggesting that students are not regarded as skilled assessors because of their lack experience, knowledge, and perspective (e.g., Arthur, 2009; Dung \& Mcinnis, 2002; Richardson, 2005) and student feedback is distorted (Yao \& Grady, 2005) (see section 2.5.4).

Proposition 8: POT may face challenges such as teachers' lack of motivation, knowledge and skills, and resources for POT. This assumption derives from reports that teachers may feel unwilling to join POT (e.g., Byrne et al., 2010), anxious and are not familiar with POT (e.g., Cairns et al., 2013; Carroll \& O’Loughlin, 2014), and do not have much time available for POT (e.g., Adshead, White, \& Stephenson, 2006; Barnard et al., 2011) (see section 2.6.5).

The way each proposition was addressed by data collection tools is shown in section 4.3.2. 


\subsection{The researcher's position}

SET and supervisory teaching observations had randomly been carried out at the study university. These tools did not affect the participants because SET was collected but its results were not returned to academics, and the observations was randomly conducted without explicit policies and structure (e.g., a few random supervisory observations a year which were not experienced by the study participants) (see section 4.1 for the research context). The recent application of SET and random teaching observation by leaders in the university and my search for academic professional development processes led me to develop an interest in the use of TT-SET and POT for academics' reflection on teaching. This study is interpretive in nature, so it is necessary for me to keep focused on my positioning as a researcher.

It is important to consider the researcher's background and perspectives in doing research - reflecting the reality. Research is filtered via the logical choice of literature review, well-developed research questions, and intuitive interpretation of findings. Although all possible attempts are made to ensure the credibility of the conclusions, the research's nature is unavoidably affected by the researcher. I cannot separate myself from the research activities in which I am intimately involved; which means "no research is free of the biases, assumptions, and personality of the researcher" (Sword, 1999, p. 277). This is because the work is interpretive in nature, and for qualitative researchers, "of all the roles, the role of interpreter and gatherer of interpretations, is central" (Stake, 1995, p. 99). Therefore, for the study to be credible, it is necessary to discuss the researcher's position. Researcher's positioning may include personal characteristics such as personal experiences, beliefs, bias, preferences, theoretical, political and ideological stance, and emotional responses to participants (Berger, 2013; Cutcliffe, 2003). According to Berger, it may affect access to the field, the nature of researcher-researched relationship, and the interpretations of findings and conclusions. It is necessary for the researcher to address the potential or actual effects of his position (Horsburgh, 2003).Thus, reflexivity, "active acknowledgement by the researcher that her/his own actions and decisions will inevitably impact upon the meaning and context of the experience under investigation" (Horsburgh, 2003, p. 309), is important for monitoring such effects (Berger, 2013). Following is the description of myself as an emerging researcher and academic of English in terms of values, beliefs, knowledge, and biases. 
My perspective of the world has been shaped by experiences including observations, conversations, formal professional training, and reading. For example, observing a colleague teaching vocabulary, I realised that she preferred using bilingual word lists to using contexts for explaining word meanings although she said students could grasp word meanings better when she provided their context of use. She explained that her students wished to know the word meanings in Vietnamese. Therefore, I have learnt that what people do might be different from what they say, and they may have different theories for what they say and what they do. Another example for reading and training is that I had taught semantics which helped me learn that, in communication, people may mean something different from the denotative meanings of their words or acts (e.g., smiling or keeping silence to show disagreement). This means that I may need different data to know the meaning people truly want to communicate.

My approach to research has been influenced by reading and a desire to gain knowledge in both quantitative and qualitative research methods. I have undertaken quantitative studies to achieve a Master of Arts in TESOL (Teaching English to Speakers of Other Languages) and a Bachelor of Arts in Finance and Banking, and I have read materials on qualitative studies. I have developed logical and intuitive ways of thinking to identify factors from data (e.g., asking why some idea was repeated by the participant, what is missing, etc.), which influenced the methodology and analysis. My approach to the data is influenced by the assumption that knowledge is socially constructed. This assumption is informed by my socio-cultural understanding of language teaching (i.e., teaching English as a foreign language) and learning in the Vietnamese context. For example, I believe that there are contextual interrelated factors (e.g., low pay, limited time, heavy workload, and poor teaching facilities) impacting on academics' learning about teaching and action. This belief is considered when I establish arguments and interpretations of the data.

Additionally, my own experience of TT-SET augmented with POT led me to want to explore aspects of this intervention. I have taught English at several tertiary education institutions for 11 years and have been in charge of quality assessment (as an adjunct to support the quality assurance and assessment unit from 2009 to 2011), research, and a teaching cohort in the department for five years (as a coordinator to support academics to follow the agenda of research projects we were doing, arrange training sessions, or group meetings where academics shared their teaching ideas). I myself 
used to collect informal student feedback, participate in POT, and coordinate academics in POT. The experiences offered me understanding of English language academics' realities and the use of SET and POT for teaching improvement. These experiences could benefit my data analysis and interpretation of findings thanks to my knowledge of the research context. My interest in doing research on the use of TTSET and POT for improvement of tertiary teaching quality derived from our first attempt at POT for the departmental academics in 2007 and recent use of SET for teaching evaluation. I was the coordinator for the teaching cohort and the process of the SET survey in the department. Therefore, I perceived the value of a collaborative teaching environment for English language academics to respond to SET and POT. These experiences contributed to my assumption that when academics regularly view their teaching through students' and colleagues' lenses, they may gain knowledge about teaching. In addition, by developing the propositions, the researcher makes explicit all the preconceptions about the intervention process: surfacing expectations whether the process works or not. I also predicted possible enabling factors and challenges to the use of the student feedback and POT. However, my deep understanding of the research context and the participants could be a limitation in a way that I could ignore providing sufficient data during analysis or siding with the participants in reporting the data. Therefore, I took this into account during data collection and analysis by taking notes about the premises of my decisions or thinking with use of a researcher journal.

I believe it is important that English language academics engage students in the learning process through:

- considering the students' discipline (or major) and their youth culture associated with their psychological patterns;

- encouraging critical and creative thinking, enthusiasm in learning new things, conceptual understanding, and collaborative learning;

- having high expectations of students' learning outcomes and building a strong learning relationship with individual students;

- encouraging self-study and reflection on learning by giving students constructive feedback of progress as well as other support;

- and using diversified teaching approaches. 
My views about teaching could affect the analysis of video-recordings of academics' actual teaching, and this is addressed by using peer debriefing.

It is my belief that academics' conscious pedagogical reasoning where reflection is the key aspect may lead to enhanced practice. For reflection on teaching through students' and colleagues' perspectives, I had previously designed my own SET form and joined in informal POT. In my case these activities have led to my deeper understanding of pedagogical theories that constitute a higher level of expertise in professional learning and practice. However, for deeper learning and effective actions to occur, it requires the necessary prerequisites such as knowledge and skills for reflection, external support, and appropriate attitudes for reflection. There are also other challenges. If teachers do not have motivation to learn from peers, they will not join in POT. This lack of motivation may result from their perception of the value and nature of the process as well as whether it is worth spending their time. In addition, I recognised a link between my own learning experiences and the use of the reflective model, the model of pedagogical reasoning and action, and social learning theory. The models align with my beliefs, and they seemed to be the logically appropriate choice for the area of study.

Furthermore, I knew each of the participants, some of which have been my peer colleagues for years. Having an established relationship with the participants had ethical and research/interpretive validity implications, which were considered and are discussed with other ethical issues in section 4.5.

I have invested my time and effort in the project for mostly four years by conducting intensive literature review, constructing a theoretical framework, identifying a research problem, sharpening the research questions, and selecting a sample for data collection. I had examined the instrumentality of case study for my research and decided which approach to case study would align with my epistemology orientation. My intention in this research project was to provide an understanding of the implementation of TT-SET augmented with POT for academics' reflection and changes to their teaching. Readers can see how academics perceive and respond to the use of students' and colleagues' lenses as prompts for reflection in their teaching process in the context of a Vietnamese university and if readers could use this model for their own context by considering contextual issues presented in the thesis. 
In conclusion, reflexivity - a major strategy for enhancing the quality of qualitative research (Berger, 2013) - is a way to scrutinise the researcher's lens. Being reflexive is to examine my own position with the study and its effects on the research context, the participants, the questions being asked, the data and their interpretation. In order to maintain my reflexivity, several strategies were used during data collection process, transcribing process, analysis and interpretation, such as member checking, triangulation, peer debriefing (peer review), and a researcher journal, which are discussed in the following chapter. 


\section{Chapter 4. Research method}

This chapter looks at the research method. First the study's context is introduced and followed by a description of the participants. Then, it describes the research procedure. Finally, trustworthiness and ethical issues are discussed.

\subsection{Institutional and programme context}

The study university is among the high-quality tertiary education institutions in Ho Chi Minh City, a big city in the south of Vietnam. It has about 350 academics and administrative staff. In 2014, the number of students was about 7,500, ranging from undergraduate to postgraduate levels. The study institution's organisation includes the Board of Rectors as top management which administers faculties, departments and centres. The Department of Foreign Languages is responsible for teaching Business English to all students. It is regarded as a supporting unit. With a large number of students, the department has to employ part-time academics in addition to a small number of tenured English language academics. Therefore, most tenured academics in the department often have a heavy schedule because they not only have to work full time in their institution, but also work part time to earn more.

Designing the Business English programme is the responsibility of the head of department and academic staff. This programme is required to conform to the national programme framework. A major Business English textbook is used with exercise books on the Test of English for International Communication. The programme is divided into two stages (general stage and professional stage), each of which has 180 periods (one period is equal to 45 minutes) distributed equally over three semesters. A course for each semester lasts 60 periods (i.e., 45 hours) over ten weeks, arranged into 20 teaching sessions (with three periods per session and two sessions on two different days per week). The department also assigns lead academics who are responsible for giving the final test after each course.

The number of students in each class may vary from 15 to 50. Academics have to prepare the mid-term and final tests which then are sent to lead academics who synthesised these tests to make a common test for each course. There are more female academics than male in the department. 
SETs and teaching observations have been used in the university. The university has designed its own standard SET questionnaires used for all academics across different majors (disciplines). These standard SETs are officially used for administration as they provide information for management only. They are not used for formative purposes, so I believe they may not serve as valuable information for academics to improve teaching. Teaching observations such as POT were informally implemented among peer academics of the Department of Foreign Languages once in 2007. Yet, none of the participants experienced such observations. In addition, I attempted TT-SET and POT in 2007 to inform my own teaching, which did not involve the participants. Recently, teaching observations have been resumed when a departmental, or faculty, or even institutional leader (such as Head of the department, Dean, or Vice-rector) makes an impromptu visit to any class (without informing academics in advance about the observation) in order to observe an academic teach. This kind of teaching observation is supervisory rather than formative. It may make academics feel that they are supervised and puts them under pressure, and they do not get much information from the observation for improving teaching. Therefore, I would like to examine the forms of SET and POT for formative purposes which allow academics freedom to choose what they need. I assume that academics respond to formative SET and POT more positively than the summative or supervisory versions.

\subsection{The participants}

The following steps allowed me to approach the participants. First of all, I made an appointment with the rector of the study university to describe the study and ask for his informed consent to conduct the research. Next, I also worked with the departmental head who could support me to contact potential participants (some of which have been my colleagues as mentioned in section 3.5), and arrange fieldwork for data collection. I contacted the potential participants via email and telephone to invite them to participate in the study. When they volunteered to participate, I arranged a meeting with them to seek their informed consent as participants in the study. I contacted 10 tenured (i.e., on a permanent or long-term contract) academics in the department with an intention to form five pairs. They all agreed to participate. However, five of them then withdrew because some said they were busy while the others were uncomfortable with an observer in their classroom. Consequently, I emailed three more tenured and four non-tenured (i.e., on a temporary or short-term 
contract) academics and invited them to participate in the study. It should be noted that these non-tenured are academics from other universities that were teaching part-time in the selected university. I had 12 volunteers, but then one withdrew from the study. Therefore, there were 11 female participants. These participants were experienced in the use of SET, compulsory POT or informal POT which were different from the formative and carefully structured TT-SET and POT in this study.

The participants were eleven English language academics, seven of whom were tenured and the other four were non-tenured. They were all involved in the same programme: teaching Business English. The criteria for selection was presented in section 3.4.1.3. Each academic selected a peer from among the participating academics for reciprocal POT, making up four pairs and a group of three peers as described in Table 4.1. The selection of participants stemmed from the assumption that academics with similar academic responsibilities would be supportive of one another in reflection on teaching (see the criteria in section 3.4.1.3). One of the goals of purposeful selection that Maxwell (2005, p. 89) mentioned is to achieve "typicality of the setting, individuals, or activities" that provide more in-depth data.

Table 4.1. The peers for peer observation of teaching ${ }^{3}$

\begin{tabular}{|l|l|l|l|l|l|}
\hline Peer pairing & Participants & Age range & $\begin{array}{l}\text { Experience } \\
\text { (years) }\end{array}$ & Qualifications & Employment \\
\hline \multirow{2}{*}{$\begin{array}{l}\text { An }- \text { Binh; } \\
\text { Binh }- \text { Cuc }\end{array}$} & An & $41-45$ & 20 & MA & non-tenured \\
\cline { 2 - 6 } & Binh & $26-30$ & 5 & MA & non-tenured \\
\cline { 2 - 6 } & Cuc & $31-35$ & 8 & MA & tenured \\
\hline \multirow{2}{*}{ Dieu - En } & Dieu & $36-40$ & 13 & MA & tenured \\
\cline { 2 - 6 } & En & $31-35$ & 11 & MA & non-tenured \\
\hline \multirow{2}{*}{ Phung - Giang } & Phung & $26-30$ & 6 & MA & tenured \\
\cline { 2 - 6 } & Giang & $36-40$ & 14 & MA & tenured \\
\hline \multirow{2}{*}{ Hang - Nga } & Hang & $26-30$ & 5 & MA & tenured \\
\cline { 2 - 6 } & Nga & $36-40$ & 13 & MA & tenured \\
\hline \multirow{2}{*}{ Dang - Khoa } & Dang & $26-30$ & 3 & MA & tenured \\
\cline { 2 - 6 } & Khoa & $26-30$ & 6 & MA & non-tenured \\
\hline
\end{tabular}

The academics' students (approximately from 15 to 40 students in each class) were those in direct contact with instructional activities, so they could detect any changes in the academics' practice. These students were not participants of the study; they just provided feedback on academics' teaching. Their feedback on teaching practice, a valuable source of data, was collected via TT-SETs. The results of early-term TT-SET

\footnotetext{
${ }^{3}$ Pseudonyms were used for the participants.
} 
were used by teachers to guide their practice with targeted areas for improvement while the end-of-term TT-SET was used to examine whether there were changes in student ratings.

\subsection{Research procedure}

\subsubsection{Data collection}

In order to gain insight into the complexity and entirety of the case, it is necessary to collect data from multiple sources (Merriam, 1998; Stake, 1995; Yin, 2009). I found it useful to follow Yin's (2009) three principles of data collection: (1) using multiple sources of evidence (for the purpose of triangulation), (2) creating a case study database (for managing data and increasing the credibility of the case study), and (3) maintaining a chain of evidence (for establishing the connections between the research questions, the data, and the conclusions).

The research question, theoretical framework, and the propositions outlined in Chapter 3 guided how data were collected for individual participants. First, participants had their own TT-SET, which was delivered early in the course for their targeting teaching aspects to be observed and improved. The same TT-SET was delivered at the end of the course for detecting changes in student ratings as an indication of whether students perceived any changes. The next source of data was from POT protocols which provided data on academics' pedagogical reasoning, particularly the reflection process. The debriefing process after POT also provided information on academics' decisions on practice, beliefs, and attitudes. The purpose of the study focuses on their perception of the intervention, so interviews were a tool to gain deep understanding of their perception of the impact, enabling factors and challenges of the intervention. Video-recordings of actual teaching before, during, and after POT provided data for independent analysis. The video-recordings were not intended to measure academics' teaching effectiveness, but as an informal tool to detect their planned changes. It was used to see if academics did what they planned as reported in their pre-observation form and peer observer's feedback form. It was developed on academics' aspirations and their planned changes.

This section presents the data collection methods and stages (see Figure 4.1 and Table 4.2 for overview). 


\subsubsection{Data collection methods}

Academics' reflection in the process of pedagogical reasoning is a complex process influenced by individual and contextual factors, so data collected from various instruments complemented each other and helped to portray the diversity and multiple dimensions of the selected academics' instructional practices. A case study design allows an examination of a wide range of evidence (Evans, 2011). Moreover, "the analysis task is to reach across multiple data sources" (Miles \& Huberman, 1994, p. 8). Therefore, this study used complementary data collection methods including: earlyterm and end-of-term TT-SETs; video-recordings of academics' actual teaching before, during, and after POT; peer observation protocols; debriefing; interviews; member checking; and researcher journal.

\section{TT-SETS}

Both early-term and end-of-term SETs were the same and tailored by each academic from an inventory of SET items (see APPENDIX A). This inventory covered a wide domain of factors evaluating teaching effectiveness. The pool of SET items was adapted from several SET questionnaires including Student Feedback on Teaching developed by the Centre for Academic Development, Victoria University of Wellington and Students' Evaluation of Educational Quality (SEEQ) developed by Marsh (1982). The questionnaire developed by Victoria University of Wellington contains both compulsory and optional parts, including fixed and open-ended questions. This questionnaire was based on Ramsden's (2003) six principles of effective teaching in higher education: interest and explanation, concern and respect for students and student learning, appropriate assessment and feedback, clear goals and intellectual challenge, independence, control and engagement, learning from students (Jones, personal communication, 3 March 2015). The validity of this SET has been establised since a study by Clift, Hall, and Turner (1989). The one developed by Marsh (1982) covers a wide range of teaching aspects. Marsh's questionnaire is a wellestablished one with multi-dimensions that has been tested and used in many universities. It is clear for academics. According to Marsh, this SEEQ measures nine distinct components of teaching effectiveness which were identified in both student ratings and academics' self-evaluations of their own teaching. Its reliability is good and the ratings have been validated with little impact from suspected sources of bias. Therefore, the questions in the inventory have been chosen with strong rationale: 
Marsh's (1982) SEEQ has been well tested for many years and Victoria's questionnaire based on Ramsden's work with its validity tested (Clift et al., 1989).

Before the start of the course, the researcher met each academic individually and gave guidelines on how they could choose items from this inventory. The aim of providing such a wide ranging choice is that teachers could identify areas that they wanted feedback on through tested questions that had been used effectively in other universities. Their TT-SET questionnaire includes both compulsory and optional parts with fixed and open-ended questions. The compulsory part provides general information about their teaching and serves as a backup in case they could not identify what teaching aspects they need to be observed at the outset of the study. The academics chose items which focus on their targeted aspects from the inventory for their optional part. The meeting with the participants focused on eliciting their desired teaching areas for student feedback. After that, each participant had their individualised student feedback form. The results from this early-term TT-SET then served as the basis for peer observation. The intent of TT-SET was not to measure teaching effectiveness, but to provide student feedback on the academics' targeted aspects of teaching. It is actually an informal form of student feedback. An example of an individualised TT-SET is included in APPENDIX B.

These TT-SETs, using a five-point Likert scale and two open-ended questions, were completed anonymously by individual academics' students. The early-term TT-SET was administered to students after four sessions. It was used to provide the participating academics with students' view of their practice so that they could identify teaching aspirations. The academic administered the TT-SET, which then was processed and returned by the researcher so that the academic could use it for reflecting and planning changes that supported the design of the peer observer's feedback form. The end-of-term TT-SET was also administered by the academic to see students' view of teaching after the intervention. The result of the end-of-term TT-SET can be motivational for academics because they may expect and use it for their future reflection on practice. It was processed by the researcher and was triangulated with other data sources (e.g., videos and reflection reports). The early-term and end-of-term SET item ratings were compared to detect changes in student ratings of academics' teaching. 


\section{Video-recordings}

Video-recordings were not used as a means of documenting actual impact of the intervention but as a means of verifying what participants perceived. These videos were used to verify academics' perceived changes in classroom performance before, during, and after POT, and to gain understanding of academics' reflection and actions (i.e., they were not intended to measure academics' teaching effectiveness but as a tool to detect if they implemented their planned changes). The videos were for two purposes: (i) the academic could review their own teaching to elaborate what they discussed with their peer during debriefing; (ii) the researcher could gain insight into what was happening during the intervention and do an independent analysis of academics' planned changes to teaching and their response to reflection. The video camera was focused on the academic, not the students. The first video-recording, conducted at the time the early-term TT-SET, was collected to capture academics' practice before POT. The second video-recording, made at the time of the peer teaching observation, was used to detect how academics acted on the planned strategies informed by the pre-observation form. The third video-recording was conducted after the second teaching observation.

The reviewers (both the researcher and his peer) of video-recordings played the role of an external observer, using the data from the three videos as a way of triangulation to examine if the academics actually reflected on teaching and did what they planned as reported in their pre-observation form and peer observer's feedback form. It should be noted that the academics also viewed the videos sent from the researcher. Examining the academics' teaching through the videos is a way of checking the reliability of these informants' account and providing a rich description of their practice. A drawback of using a camera to record academics' actual teaching may be that academics just do what they thought was expected just because the camera was there. However, the academics had a plan in advance for their teaching which was written in their reflection form.

\section{POT protocols}

Peer observation protocols were designed for reports on teaching observation and used as evidence for what was going on in the POT process. The POT protocol was used to guide the academics because they were first-time participants of the intervention. After 
the early-term TT-SET was collected and processed, the results were given to academics. These results were to help them identify teaching areas that they wanted to improve. A pre-observation meeting was arranged for every pair of peers, and they were given a pre-observation form (see APPENDIX C) developed by the researcher which included questions on: areas in which their students wanted them to change; areas the academics felt they needed to improve and on which they needed feedback from a colleague, and strategies they would use as well as the reason for using them. The basis for the formation of this pre-observation form lies in the purpose of TT-SET and POT (i.e., based on the academics' targeted aspects of teaching to be improved).

The researcher adapted a pre-observation form developed by Chism (2007) by selecting the questions that suit this purpose. In addition to describing peer review of teaching comprehensively, Chism includes resources, sample forms and detailed instruction on conducting classroom observations and effective ways to document them. She provides detailed guidelines on POT in the process of peer review of teaching, including guidelines on preparation for POT, peer observation log, and reflective narrative. These guidelines are well-developed and appropriate for the purpose of POT in this study. For example, Chism's guideline for peer observation for formative purposes includes narrative prompts for what is observed and suggestions for enhancement. The guideline is clear and relevant to the development of the peer observer's feedback form in accordance with the purpose of POT in this study. Therefore, these forms were adapted to serve the purpose of POT. Because the study focuses on academics' changes in pedagogical reasoning, the adapted POT protocols also included issues related to pedagogical reasoning. For example, the preobservation form includes questions of areas academics want to be observed and improve, the changes they are going to make and strategies.

Based on the information on the pre-observation form, the pair of peers developed a peer observer's feedback form which was used by the peer observer when observing their colleague's teaching. This form consisted of items or areas of teaching for feedback agreed upon by both peers. In other words, the peer observer's feedback form was developed based on what academics wanted to improve and wanted their peer to observe, thus during observation, the peer gave comments on these targeted areas and offered suggestions for changes (if any). 
After the observation, a teacher's reflection report form (see APPENDIX C), adapted from Chism (2007), was given to the academics. The basis for the development of this form lies in purpose of POT and the research questions. Thus, relevant questions from Chism's reflective narrative were selected to form the teacher's reflection form to explore the changes that had occurred in academics' pedagogical reasoning. This reflection form included questions for academics' reflection and their perception of the values of each step in POT, such as "What have you learned about your teaching as a result of this POT process? What will you definitely keep in your teaching repertoire? Why? Focus on 1-3 aspects of your teaching. What will you most likely change about your teaching approach? Why? How? What were especially useful and how may they lead to improvement of your classroom teaching. List any future agenda items that have emerged as a result of the POT process."

\section{Debriefing after POT}

The debriefing session with both academics as a pair of peers was carried out after the academics had finished their first POT. The researcher was a facilitator of the debriefing to remind the academics to follow the POT protocols and stay focused during their limited time for debriefing. The guiding questions included "What do you think about the student feedback and how does it affect your choice of items for POT? What challenges have you faced and how can you improve your teaching? Why did you choose the items for POT? What changes in teaching are you going to make?" (see APPENDIX D). Questions were asked during their conversation about teaching to explore their perception of the intervention, their teaching beliefs, experience, and aspirations for improvement. These questions were based on the purpose of the debriefing and POT. As the pre-observation meeting provided a chance for academics to talk about what they wanted their peer to observe, the post-POT dialogue was about the changes they planned to make, why they wanted to make changes, on what basis they based their decisions, and any perceived impact of previous experience, training, etc. associated with TT-SET and POT. The purpose of these questions was to focus academics' conversation. The audio-recording of the debriefing session was used to capture areas of reflection and learning in the process of pedagogical reasoning after POT and developmental strategies for instructional improvement. 


\section{Interviews}

A well-conducted interview is an effective way to collect rich data on people's perspectives, attitudes, and the meanings underlying their lives and behaviours (Gray, 2014). The purpose of interviewing is to help the researcher uncover what could not be observed, enter into the participants' perspectives and discover the meaning attached to their own experiences (Merriam, 2001; Patton, 2002). Interviews were conducted with individual academics at the end of the course they were teaching to capture areas of reflection and learning in the process of pedagogical reasoning after the intervention, to gain deeper understanding of their behaviours, and to explore their perception of the value of TT-SET and POT for their reflection. The interviews aligned with the exploratory nature of this study because they were designed to "explain and account for the descriptive information" and address the questions 'why' and 'how' (Gray, 2014, p. 36).

Interview questions were developed to align with the research questions and variables of interest emerging from the literature review. Open-ended semi-structured interviews, with a list of guiding interview questions about the research focus or issues, were used to gather data from the academics about their perceptions of the intervention process. In other words, the basis for forming these questions is informed by the issues to be explored (see APPENDIX E). These open-ended semi-structured interviews were used because of the assumption that "individual respondents define the world in unique ways" (Merriam, 2001, p. 74). They align with the interpretive nature of the study. The wording and order of the questions were altered according to the flow of each interview; this flexibility allowed the researcher "to respond to the situation at hand, to the emerging world view of the respondent, and to new ideas on the topic" (Merriam, 2001, p. 74). Additional questions were also developed for further information in case their answers needed further clarification. Thus, the interview guide contained several specified questions, some more open-ended questions (possible probes), and a list of issues to be explored. The individual interviews were conducted in Vietnamese, which guaranteed the participants' understanding of content and ease in communication. Each interview lasted from 45 to 60 minutes. These interviewees were allowed sufficient time to think and answer each question and were asked to confirm the meaning they gave. The interviews were audio-recorded to preserve all information for analysis (Merriam, 2001). For a natural conversation, an appropriate climate for 
the interview was created. Because all the participants were my colleagues and acquaintances, the interview started with questions for information about their work or family so that they could feel relaxed. The interviewer-interviewee relationship was made clear to the participants, so the credibility of responses can be maintained. This ethical dimension is discussed in section 4.5.

\section{Member checking}

For the credibility of information elicited during the interview, member checking was used. Member checking is one of the ways of enhancing the credibility of a qualitative study (Creswell \& Miller, 2000). As Creswell and Miller described, data and interpretations are sent back to the study's participants in order to "confirm the credibility of information and narrative account" (p. 127). During an interview, the researcher restated or summarised interview information and then questioned the participant to determine its accuracy. The interviews, which were conducted in Vietnamese, were transcribed by one person who signed a confidentiality agreement with the researcher (see APPENDIX F). These transcripts were then checked by the researcher for missing words, slang, or terminology mistaken by the transcriber because academics sometimes used English words in the interviews which made it difficult for the transcriber to follow. Then, data transcripts in Vietnamese were sent back to the participants for confirmation of their accuracy. Member checking was also employed for the logic models developed during data analysis, which summarises the participant's experience of the intervention. They were sent back to them for clarification (i.e., the researcher asked them if they could understand the logic model and confirm the accuracy of the information presented).

\section{Researcher journal}

A researcher journal was used to keep a detailed record of the researcher's own thoughts, and new ideas, reasons for decisions, and interpretations during the process of data collection and analysis. This was done to help reduce bias and capture important issues for later use. It was also used for keeping notes during the debriefing session and interviews. As Corbin and Strauss (2008) noted, "Field notes are data that may contain some conceptualization and analytic remarks" (pp. 123-124). The researcher journal was also used to note any new ideas that occurred and were useful for the analysis and discussion. 
A summary of the data collection methods and purposes for this study is presented in Figure 4.1.

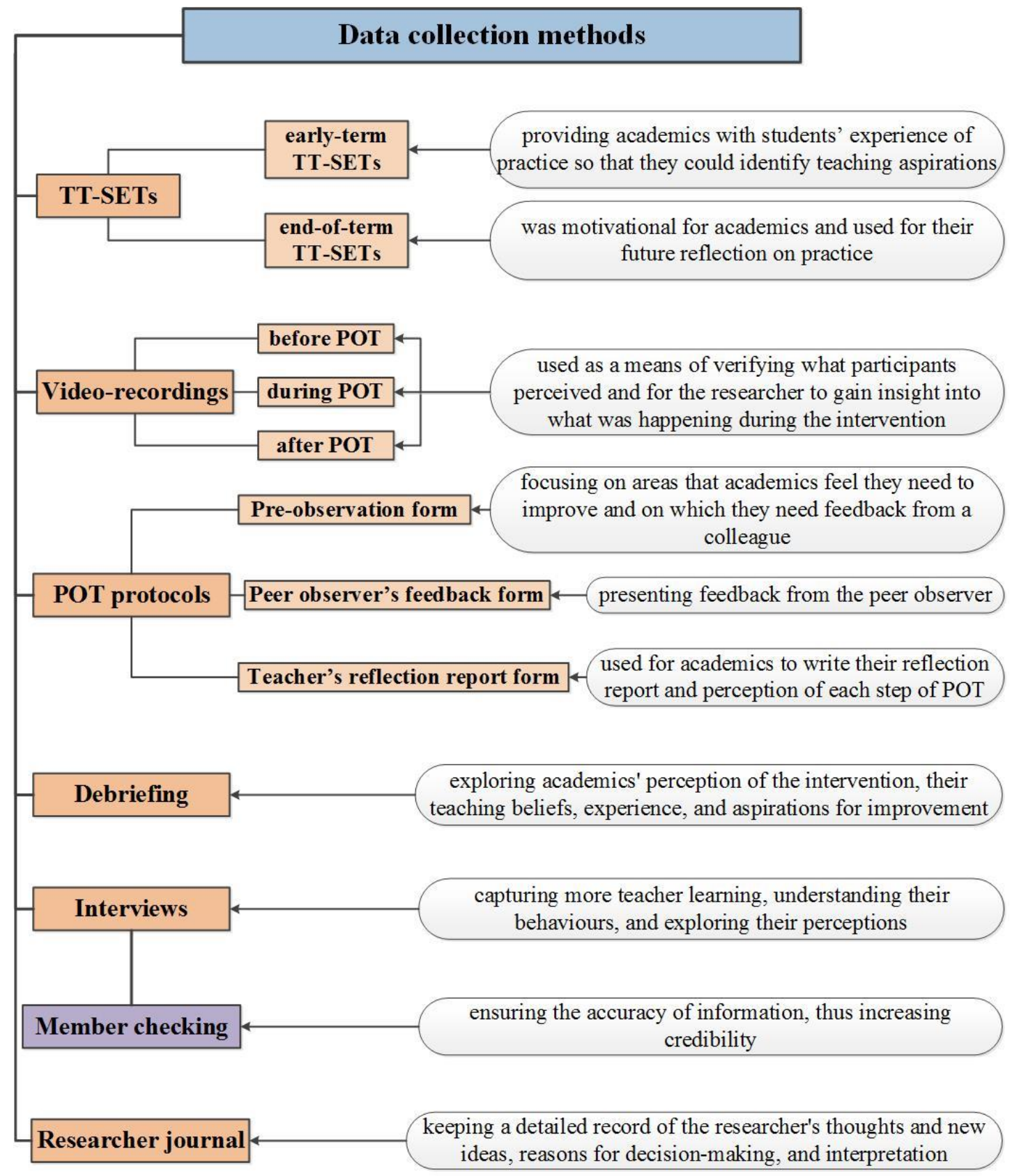

Figure 4.1: Data collection methods

\subsubsection{Data collection stages}

Data collection activities were implemented through seven stages throughout one semester, including interventions. Although the timetable was set up in advance for the data collection process, the schedule changed when I reached the field. First, the course started and ran on for two weeks before Tet holidays (Lunar New Year, 
important public holidays in Vietnam). After the three weeks, the course resumed. Second, a new regulation made the schedule flexible for academics in a way that they could lengthen the course to 12 weeks. Eventually, all the participants arranged their schedule for 12 weeks so long as they taught for 20 sessions. The actual data collection (as shown in Table 4.2) therefore changed as compared to the proposed one.

Table 4.2: Collection stages of the case study

\begin{tabular}{|c|c|c|c|}
\hline \multicolumn{2}{|c|}{ Time } & \multirow{2}{*}{$\begin{array}{c}\text { Stages } \\
\text { (1) }\end{array}$} & \begin{tabular}{|ccc}
\multicolumn{3}{c}{ Intervention } \\
ouidelines on neer selection and & TT-SET
\end{tabular} \\
\hline & & & $\begin{array}{l}\text { guidelines on peer selection and TT-SET } \\
\text { development for individual academics }\end{array}$ \\
\hline \multirow[t]{2}{*}{ Week 1} & Session 1 & & \\
\hline & Session 2 & & \\
\hline \multirow[t]{2}{*}{ Week 2} & Session 3 & & \\
\hline & Session 4 & (2) & early-term TT-SETs, pre-POT video-recording \\
\hline \multicolumn{4}{|c|}{ Lunar New Year holidays } \\
\hline \multirow[t]{2}{*}{ Week 3} & Session 5 & & \\
\hline & Session 6 & \multirow[t]{2}{*}{ (3) } & \multirow[t]{2}{*}{ reciprocal POT1, while-POT video-recording } \\
\hline \multirow[t]{2}{*}{ Week 4} & Session 7 & & \\
\hline & Session 8 & (4) & post-POT debriefing \\
\hline \multirow[t]{2}{*}{ Week 5} & Session 9 & & \\
\hline & Session 10 & & \\
\hline \multirow[t]{2}{*}{ Week 6} & Session 11 & & \\
\hline & Session 12 & & \\
\hline \multirow[t]{2}{*}{ Week 7} & Session 13 & \multirow[t]{5}{*}{ (5) } & \multirow[t]{5}{*}{ reciprocal POT2, post-POT1 video-recording } \\
\hline & Session 14 & & \\
\hline \multirow[t]{2}{*}{ Week 8} & Session 15 & & \\
\hline & Session 16 & & \\
\hline \multirow{4}{*}{$\begin{array}{l}\text { Week } 9 \text { - } \\
\text { Week } 12\end{array}$} & Session 17 & & \\
\hline & Session 18 & & \\
\hline & Session 19 & (6) & end-of-term TT-SETs \\
\hline & Session 20 & (7) & interviews with participants \\
\hline
\end{tabular}

Stage 1 (before the start of the course): The researcher offered the participants guidelines on choosing a peer from the same discipline for POT (i.e., they chose their peer and then were invited to participate in the intervention) and developing a TT-SET questionnaire from an inventory of items carefully collected from well-established SETs.

Stage 2 (Week 2 -Session 4): The academics were asked to distribute the self-tailored SET forms to their students for feedback on their teaching at the end of session 4 . The researcher processed the data and produced the feedback results. The academics' teaching was video-recorded. The video was sent to them to view.

Stage 3 (Week 3 - Session 6): After receiving student feedback, the participants were oriented to a pre-observation meeting (recorded via a pre-observation form). They 
planned their teaching and discussed the areas they wanted their peer to observe (by developing an observation form).

The participants were guided to carry out the reciprocal POT process in session 6 or 7 and were asked to be mindful of collegiality, confidentiality and purposeful enhancement of teaching quality. The observer was instructed to observe the teaching without obtrusiveness or interference. The academics attended each other's teaching for the whole class session. The peer observers completed a peer observation form during or after the session. The academics' teaching was video-recorded. The video was sent to them to view.

The observed academic then received the confidential written feedback from their peer and had teaching dialogue with the person in a post-observation meeting so that the teacher could reflect on their teaching based on the feedback. Because the researcher was present at the debriefing, he committed to keep the confidentiality of the dialogue as well.

Stage 4 (Week 4 - Session 7 or 8): The post-POT meeting was flexible and could be arranged immediately after POT or in week 8 , depending on the availability of the peer academics. At the debriefing session, the researcher facilitated the discussion on teaching between the peers. The researcher also joined the discussion by asking the academics and their peers about the areas of practice they were going to change and what governed the changes they were going to make.

Stage 5 (Week 7, 8, or 9 - Session 13-17): POT2 was carried out to see how the planned changes were made in the classroom. Because the academics had heavy workloads, they could not arrange the second POT after a week as the researcher planned, they participated in the reciprocal POT during week 7,8, or 9, depending on their arrangement with the peer. The peer observer and the observed academic wrote their reports. The peers gave each other their observation reports. The academics' teaching was video-recorded. The video was sent to them to view.

Stage 6 (Week 11 - Session 19): The participants again delivered the same TT-SETs to students to see if there were changes in students' ratings of teaching.

Stage 7 (Week 12 - Session 20): Stage 7 was flexible, depending on the availability of the academics. After the participants finished their $20^{\text {th }}$ teaching session, the researcher arranged time with individual academics for interviews. The researcher sent 
the participants a list of the questions to provoke their thinking relating to the effectiveness of TT-SET augmented with POT and related issues to be explored. The list of questions was used as a guideline for the interview. The researcher interviewed the participants, using these questions as a guide to gain deeper understanding of their perceptions.

\subsubsection{Relationship of data to research questions and propositions}

This section shows the links between the data methods and the research questions with propositions. Table 4.3 shows how the sources generate data in relation to the research questions and the propositions.

Table 4.3: The sources that generate data in relation to the research questions and the propositions

\begin{tabular}{|c|c|c|c|c|c|c|c|}
\hline Research questions and propositions & Data methods & 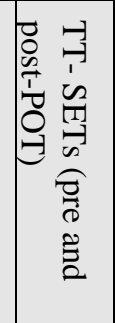 & 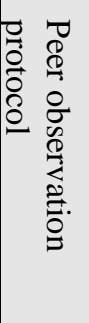 & 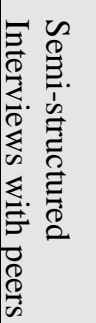 & 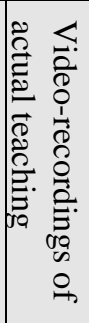 & 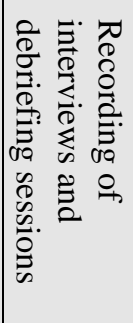 & 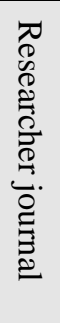 \\
\hline \multirow{3}{*}{$\begin{array}{l}\text { Main question: In the context of a } \\
\text { Vietnamese university, how does TT- } \\
\text { SET augmented with POT impact on } \\
\text { academics' pedagogical reasoning? }\end{array}$} & Proposition 1 & & $\checkmark$ & $\checkmark$ & $\checkmark$ & $\checkmark$ & $\checkmark$ \\
\hline & Proposition 2 & & $\checkmark$ & $\checkmark$ & $\checkmark$ & $\checkmark$ & $\checkmark$ \\
\hline & Proposition 3 & $\checkmark$ & $\checkmark$ & $\checkmark$ & $\checkmark$ & $\checkmark$ & $\checkmark$ \\
\hline \multirow{2}{*}{$\begin{array}{l}\text { Sub-question 1: What is the } \\
\text { perceived impact of TT-SET } \\
\text { augmented with POT on academics, } \\
\text { practice, beliefs, and attitudes? }\end{array}$} & Proposition 4 & & $\checkmark$ & $\checkmark$ & $\checkmark$ & $\checkmark$ & $\checkmark$ \\
\hline & Proposition 5 & & $\checkmark$ & $\checkmark$ & $\sqrt{2}$ & $\checkmark$ & $\checkmark$ \\
\hline $\begin{array}{l}\text { Sub-question 2: What are academics, } \\
\text { perceptions of the effectiveness of } \\
\text { TT-SET augmented with POT for } \\
\text { promoting their reflection in the } \\
\text { process of pedagogical reasoning? }\end{array}$ & Proposition 6 & & & $\checkmark$ & $\checkmark$ & $\checkmark$ & $\checkmark$ \\
\hline \multirow{2}{*}{$\begin{array}{l}\text { Sub-question 3: What are possible } \\
\text { challenges to the implementation of } \\
\text { TT-SET augmented with POT in } \\
\text { Vietnam's context? }\end{array}$} & Proposition 7 & & & $\checkmark$ & & $\checkmark$ & $\checkmark$ \\
\hline & Proposition 8 & & $\checkmark$ & $\checkmark$ & & & $\checkmark$ \\
\hline
\end{tabular}

(*) See section 3.4.2 for details of each proposition

\subsubsection{Data analysis}

Merriam (2001) regards data analysis as "the process of making sense out of the data. Making sense out of data involves consolidating, reducing, and interpreting what 
people have said and what the researcher has seen and read - it is the process of making meaning" (p. 178). The following analytic strategies and techniques were employed.

Three of the four strategies proposed by Yin (2009) were used for data analysis. The first strategy was developing an embedded case description. The descriptive approach was used to describe the embedded cases and the complexity held within them (Yin, 2009). This means this descriptive approach was used for describing 11 embedded cases and an overall pattern of complexity to describe and explain the nature of each teacher's perceptions about the intervention and how these related to the teacher's response and subsequent actions. The description included a range of themes, telling the story of the participant engaging in SET and POT. The analysis started with a description of participants' experience of the intervention as the basis for a comprehensive understanding of the practice of TT-SET and POT as a whole.

The second strategy used both qualitative and quantitative data because the data are related to an embedded case within the broader single case study and cover the outcomes of the intervention (Yin, 2009). In addition to qualitative data from the observation protocol (pre-observation forms, peer-observer's feedback forms, teacher's reflection report forms), debriefing and interview transcripts, and the researcher journal, the quantitative results of early-term TT-SET and end-of-term TTSET were compared using SPSS software to see if there were changes in students' ratings of teaching. After calculating the mean scores and standard deviations from the early-term and end-of-term SETs, paired-samples t-tests were utilised to determine if the differences were significant (confidence interval is $95 \%$, or alpha is equal to 0.05 ).

The third strategy was examining rival explanations during cross-participant analysis. It is used to analyse the data for emerging themes and explain why there are differences (if any) between participants. Considering and ruling out other possible plausible explanations is a way to validate the findings. This can be achieved by providing sufficient evidence. It should be noted again that the literature on TT-SET and POT as formative lenses for teachers' reflection was reviewed and discussed prior to conducting data collection to provide the researcher with insights into the development of intervention processes and a sound grounding in present debates, concerns, practices, and trends in the world including Vietnam. The review of literature served as a reference for discussion when analysed data were compared to confirm or refute the information from the literature. 
The analytical techniques used in this study included logic models, cross-(embedded) case synthesis, and explanation building as proposed by Yin (2009). A logic model was developed during analysis to give a full picture of each participant's experience of the intervention. The logic models were intended to show interconnection between the intervention and a certain outcome or sequence of outcomes. The logic model was basically a description and may not describe everything and may be in danger of being oversimplified. Readers may not see a full picture of the whole case because it is sometimes hard for them to make connections of elements presented in the logic models for individual participants (Merriam, 2001). Thus, cross-(embedded) case analysis would be useful. This cross-participant synthesis (using thematic analysis) was to complement the logic model. In using this kind of synthesis, it is suggested that "the examination of word tables for cross-case patterns will rely strongly on argumentative interpretations, not numeric tallies" (Yin, 2009, p. 160). Therefore, the data were organised to support plausible explanations for the outcomes of the intervention among the academics. The analysis across participants was used to answer the research questions. Then, explanation building resulted from seeking for the similarities and differences between and across the embedded cases. This study was exploratory (i.e., the goal is not to draw conclusions but to develop ideas for further research) (Yin, 2009). Explanation building was to examine other possible explanations identified during cross-participant synthesis, and the data were reviewed to see if there are any specific conditions to explain differences (if any) among participants.

\subsubsection{Analysis of individual participants}

Each individual participant's experience of the TT-SET augmented with POT was reported following a similar format. While the theoretical framework to identify interconnections between important elements in the scope of the research emerged from the synthesis of the literature, a logic model was developed during the analysis of the individual participants nested in the single case study, providing a consistent format for analysis. Embedded in the logic model were the research questions, each of which included variables of interest (or themes). The logic model was designed to summarise the key beliefs and experience that may have affected the academic's teaching practice and aspirations for teaching improvement. It also described various factors the academics experienced and that may have enabled or hindered their 
reflection for improvement of teaching quality through the TT-SET augmented with POT. Following these enablers and barriers were academics' new comprehension and action. Examining individual participants' experience of the intervention helped gain understanding of the complexity of its implementation. The logic model was used to provide a framework for "thick" descriptions and deep understanding of the individual participants and to inform the thematic analysis that followed.

\subsubsection{Data coding and analysis procedures}

The data from the sources such as peer observation protocols, debriefing, interviews, and researcher journal were coded directly in Vietnamese to maintain their original meanings. Next, the data in Vietnamese were translated into English for phrases, sentences, or paragraphs to be directly quoted in the analysis and findings chapter in the thesis by the researcher, who has three years' experience of translating. The researcher's knowledge of translation and experience in translation contributes to the trustworthiness and objectivity (equivalence) of the translation. In addition, these translations were randomly checked for consistency by a peer who is fluent in Vietnamese and English.

The process of data analysis followed the steps adapted from Creswell (2009) as shown in Figure 4.2. 


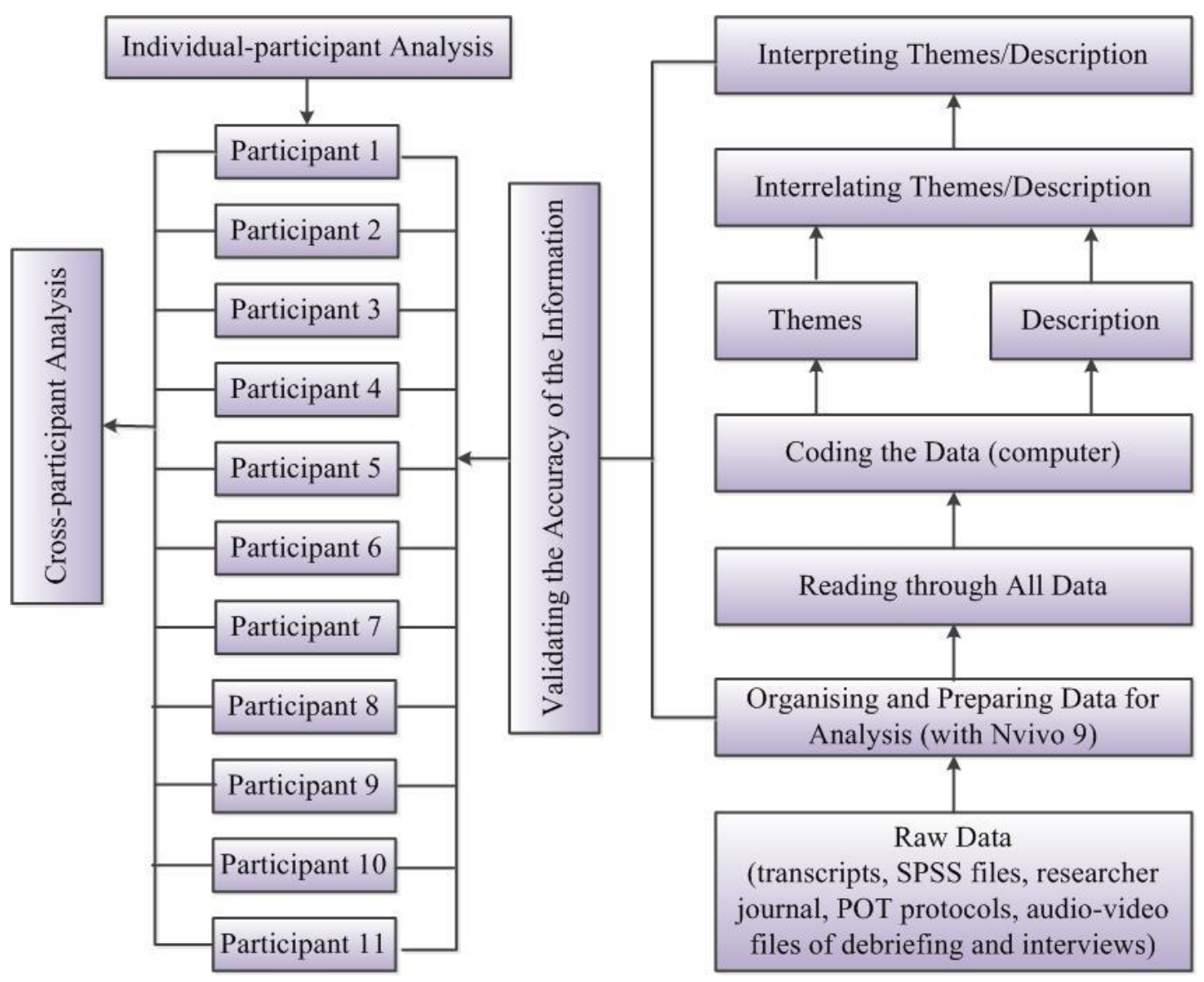

Figure 4.2: Data Analysis in Qualitative Research adapted from Creswell (2009)

The data analysis followed an inductive approach. First of all, I read through the TTSET results, POT protocols, debriefing and interview transcripts, and the research journal. I also listened to audio-recordings of the debriefing and interviews and watched all video-recordings of academics' actual teaching. Reading and listening to the information allowed initial thoughts about organising and preparing the data for analysis. This initial stage of analysis offered me an overview of the whole before breaking data down into bits of information (Maxwell, 2005). This thorough review of the data allowed the data to be broken into meaningful parts or bits of information and assigned to codes. These multiple codes were compared and contrasted and sorted into groupings that have something in common (Merriam, 2001), forming groups of categories (e.g., enhanced reflection, perceived changes, enhanced confidence and efficacy, enhanced collaboration and collegiality). I also referred to the definition of related concepts from the literature and established their distinctive features in order to decide which quotes would fit into which categories. My judgment on which quotes that fit into the codes depended on not only the meaning of a single piece of information but also the meaning implied by the participants from varied data and 
member checking. These descriptive categories allowed me to gain easy access to information in the analysis and interpretation phase (Merriam, 2009). At this early stage, I actually started examining themes across the categories (e.g., positive outcomes). By going through the data set multiple times and reviewing the coding, I formulated codes that were then condensed into categories and the themes were identified across categories. By reviewing data and coding many times - "a constant moving back and forward between the entire data set" (Braun \& Clarke, 2006, p. 86), I better grasped the categories or concepts that had emerged. Therefore, in some cases, new ideas developed and required additional coding, resulting in new categories and a re-analysis in the light of new themes. Again, themes across categories were then identified and grouped for common themes, which were assigned to conceptual labels that underpinned data interpretation. The analysis involved my intuitive, logical, and critical thinking with questions (e.g., How were the themes interrelated? How they answered the research questions? What aspects were being captured and what was missing? Which themes made meaningful contribution to the understanding what happened within the data?) (for examples of coding, see APPENDIX G). This inductive approach allowed the data to be constructed into themes (Miles \& Huberman, 1994) independent of those initially identified from the literature review and the pre-determined propositions that had been used to shape the data collection plan.

It is noted that the categories were then related to each other through combination of inductive and deductive thinking (Merriam, 2001). A large part of the qualitative data analysis was inductive. However, as the researcher progressed in analysing the data, "tentative categories, properties, and hypotheses continually emerge and must be tested against the data - that is, the researcher asks if there are sufficient data to support a certain category or hypothesis" (Merriam, 2001, p. 192). The construction of categories also reflected the focus of the study and the research questions (Merriam, 2001). Therefore, when a category lacked sufficient data and was not related to the study focus, it would be left out. In addition, comparing the themes helped identify strong themes and the relationships between themes emerging across multiple data sources to organise them into categories and sub-categories (if any). When ideas fitted into a structure of generic relationship, the relationship of categories formed the layers of the analysis. These layers helped develop a report framework with the identified 
categories or themes acting as headings or subheadings of the report (Stringer, 2007). The themes were analysed for individual participants and across participants. The analysis included answering the research questions across the participants in themes or variables of interest (Miles \& Huberman, 1994).

Exploring and refining the data analysis framework involved logical, intuitive, and critical thinking about the meaning, the applicability, the importance, and underlying connections between ideas. Discussion of interconnecting themes conveyed the findings of the analysis. Interrelating the findings or themes facilitated the interpretation of their meaning through comparing the findings with information from the literature and related theories, leading to consideration of whether these findings concurred or diverged from past information and whether new questions were raised by the data which had not been predicted. The analysis of individual participants, together with thematic analysis, allowed an exploration of how TT-SET augmented with POT facilitated academics' reflection and action to improve teaching. This rigorous procedure of analysis ensured that the research questions were being fully addressed. Eventually, a summary was drawn as a result of the analysis and synthesis.

\subsubsection{Data management}

For data management and coding, the QRS NVivo 11 software programme was used because a computer-assisted qualitative data analysis software "may serve as an able assistant and reliable tool" (Yin, 2009, p. 128). In this study, there were a lot of data from many data collection tools such as debriefing transcripts, interviews transcripts, POT protocols and researcher journal. NVivo made data easily retrievable. It facilitated a systematic way of storing the data, categorising them into topics, and coding them into themes under nodes. It helped to see the link between codes representing these themes and retrieve the data quickly. The software allowed changes (e.g., adding, merging, moving, coping, etc.) to be made to nodes when grouping or regrouping categories. A database for each participant was also created and arranged for analysis across participants. Carefully managing the data added rigour to the way they were processed, thus increasing research trustworthiness. Hard copies of POT protocols were stored in a flip folder while other electronic files including SPSS files of TT-SET results, audio-recordings of debriefing and interviews, and videorecordings of actual teaching were retained in back-up files in my computer and portable hard drive. 


\subsection{Trustworthiness of the Study}

Trustworthiness of a study is essential to evaluating its worth (Lincoln \& Guba, 1985). To establish trustworthiness, it is necessary to: (1) enhance the confidence in the truth of the findings (i.e., credibility), (2) show that the findings are consistent and could be replicated (i.e., dependability), (3) clarify the extent to which the findings of a study are shaped by the respondents and not researcher biases (i.e., confirmability), and (4) show that the findings can be applied to other contexts (i.e., transferability). The following subsections will discuss the procedures to maintain the trustworthiness of the study.

\subsubsection{Credibility}

There is general consensus that qualitative researchers need to demonstrate the credibility of their research (Creswell \& Miller, 2000). To enhance the credibility of qualitative research, a number of procedures have been used by researchers. Creswell (2009) suggested the employment of multiple strategies to maintain the accuracy of the research findings such as triangulation, member checking, external auditor reviewing, presenting negative and discrepant information, spending prolonged time in the field, using rich and thick description, being reflexive and clarifying bias, collaboration, and peer debriefing. Based on the nature of this study, several procedures were taken to enhance its credibility.

First, rich and thick descriptions were used. This study was conducted with varied data sources from lecturers and students through different instruments (TT-SET forms, video-recordings, observation protocols, debriefing, interviews, and research journals) which then provided many perspectives about a theme. Second, triangulation was used. The benefit of the case study method for social inquiry is the facilitation of triangulation. Triangulation can be corroborative, or it can ensure that the whole picture is gained.

Stake (1995) and Patton (2002), discussed four types of triangulation: data triangulation (the triangulation of data sources); investigator triangulation (triangulation from different evaluators); theory triangulation (triangulation of perspectives on the same data set); and methodological triangulation (triangulation of results from different methods). Data from the aforementioned sources (e.g., students and teachers) were compared and contrasted to ensure the logic of interpretation (i.e., 
"to establish a high level of harmony between the participants' expressions and the researcher's interpretations of them" (Jensen, 2008b, p. 139)). Triangulating data across varied sources helps "build confidence in the precepts of a theoretical proposition" (Evans, 2011, p. 59).

Different evaluators were also involved in interpreting the data. For example, in reviewing academics' actual teaching in the video-recordings, a peer was invited to interpret the videos based on a defined guideline. A review sheet for video-recordings is included in APPENDIX H. A clearly-defined set of criteria was developed based on teachers' targeted areas of improvement, and given to a peer reviewer who is an English language academic in order to find evidence supporting changes in areas targeted for improvement. Video reviewers played the role of external observer while reviewing videos. The purpose of the videos was to detect how academics acted on planned changes informed by the pre-observation form. The review is descriptive and used to complement other sources of data. The measure was developed based on what academics wanted to improve, their stated strategies (as presented in Part 1 of the video evaluation sheet), and things they learned from the first POT (Part 2). Then, the targeted items were listed again in Part 3 followed by prompt questions for evaluation (Part 3). Based on reading the three parts 1,2, and 3, the video reviewer commented on academics' teaching through the pre-POT, while-POT, and post-POT videos (Part 4). Finally, he/she wrote overall comments on academics' teaching. The reviewer through the video-recordings played his/her roles as an external observer who provided comments on the academics' teaching. My peer and I reviewed the same videos. Then, the peer's comments were compared with mine for the credibility of the data interpretation. This peer also cross-checked the researcher's translation of direct quotes from participants. Advice from my supervisors was included in the interpretation of data, forming a type of investigator triangulation which enhances the reliability of the interpretation. The interpretation of the data set was made with reference to different theoretical perspectives from the literature review.

Method triangulation strengthens data credibility and trustworthiness in qualitative research (Patton, 2002; Yin, 2009). The seven data methods were described in Figure 4.1. In addition, another procedure to enhance credibility of interpretation is to selfdisclose the researcher's assumptions, beliefs, and biases. This is researcher reflexivity, as presented in section 3.5. This allowed readers to understand the 
researcher's position and bracket the biases (Creswell \& Miller, 2000). Finally, member checking was used when transcripts were sent back to participants for confirmation of their accuracy. The logic model that summarises participants' experiences of the intervention was sent to them for confirmation. It is a way to make the information credible (Creswell \& Miller, 2000).

\subsubsection{Dependability}

Dependability emphasises "the researcher-as-instrument and the degree to which interpretation is made in a consistent manner" (Baxter \& Eyles, 1997, p. 517). It implies that if the researcher's procedure and instruments to study a phenomenon are applied by others in similar research conditions, similar findings should be yielded (Given \& Saumure, 2008). Baxter and Eyles (1997) stated that dependability, "the degree to which it is possible to deal with instability/idiosyncrasy and design-induced change..., includes the consistency with which the same constructs may be matched with the same phenomena over space and time" (p. 516). Baxter and Eyles also indicated that the dependability of interpretations in qualitative research can be threatened by poorly defined analytical constructs and premises and premature closure. The dependability of this case study was obtained by employing the same procedures of data collection and analysis (Yin, 2009) for all subjects/individual participants. For the accuracy of data collection and interpretation, all necessary information was preserved through researcher journals (including field-notes), video-recordings of teaching, and audio-recordings of interviews. Particularly, the researcher journal was used to remind me of new ideas that arrived during the analysis process. In addition, the interview transcripts, data descriptions, and data analysis (e.g., the logic model developed during analysis was sent back to participants in order to see if the interpretation reflects what participants meant) were verified by the actual participants (Patton, 2002). For data analysis, peer examination or debriefing was used to enhance the accuracy of the process of coding, identifying emerging themes, and interpreting data. As Suong (2008) stated, peer debriefing is a process whereby the researcher has a peer to probe his or her thinking around all or parts of the research process such as methodology, interpretation, and data analysis. The research findings and conclusions were shared with my colleagues, supervisors, and other experts in the field to detect problems such as misinterpretation, unconvincing explanations, and researcher bias. As described previously, for example, a colleague was invited to analyse the video- 
recordings of academics' teaching and made a translation of the interview transcripts. This procedure for data collection and analysis should yield reliable and convincing findings.

\subsubsection{Confirmability}

Confirmability, the extent to which the research's results are based on its purpose and not distorted by the researcher's bias, contributes to the trustfulness of a study when assertions are verified (Jensen, 2008a). Baxter and Eyles (1997) stated: "Confirmability, similar to the conventional notion of objectivity, focuses attention on both the investigator and the interpretations" (p. 517). Confirmability requires the researcher to make explicit the potential sources of bias in his or her personal and professional information (e.g., experience, training, prior knowledge, values, beliefs, etc.) for the investigator, being the instrument in qualitative inquiry (Patton, 2002). My experiences, motivations, formal English language training, and perceptions of an English language academic in Vietnam might have affected the interpretation of data and the problem under study, so the researcher's perspectives were made explicit (see section 3.5). To reduce researcher bias in this study, it was also necessary to consider these with critical self-reflection (Patton, 2002) by writing researcher journals to disclose my perspectives in collecting, interpreting, and analysing data (see section 4.3.1.1).

\subsubsection{Transferability}

Transferability implies that a qualitative study's results "can be transferred to other contexts and situations beyond the scope of the study context" (Jensen, 2008c, p. 887). For transferability to be enhanced, Jensen suggested that much attention should be paid to "how closely the participants are linked to the context being studied and the contextual boundaries of the findings" (p. 887). Therefore, the qualitative researcher is responsible for a comprehensive description of the study's context so that readers can determine if the study's results can be transferred to their context. The objective of this study was not to make generalisation of the research results to other populations or contexts. This study centred on in-depth understanding of a particular phenomenon; the value of qualitative research lies in the "particularity rather than generalizability" (Creswell, 2009, p. 193). Nevertheless, this thesis also provided rich, thick description 
(Merriam, 1988) of the research context and methodology so that those interested in transferability can relate the study's insights to similar research in their own context.

\subsection{Ethics}

Prior to the commencement of the study, the research proposal was sent to the Faculty of Education Ethics Committee, Victoria University of Wellington for ethics approval. This research has been assessed and approved by the Faculty of Education Ethics Committee, Victoria University of Wellington (No: SEPP/2012/88 RM 19615) (see APPENDIX I). It was essential to ensure ethical treatment of the research participants so that the study did not involve any deception and was not designed to be highly intrusive to their lives. Respect for the participants and sites needed to be shown (Creswell, 2009). Ethical principles were strictly kept throughout the research process.

First of all, a letter and a written informed consent were sent to the rector (see APPENDIX $\mathbf{J}$ and APPENDIX K), the department leader and all participants in the university where the research was to be carried out. Then, reciprocity between the researcher and the participants was enhanced with discussion on the purpose of the study, the schedules of data collection, the locations of the interviews, and anonymity protection. The participants were assured of confidentiality of the information they provided: security of information during the processing, analysing, and reporting of findings. Identifying information was removed in the thesis and in a related professional conference presentation, and will be from journal articles. There was also a need to seek peer observers' assurance that they would respect the peers' confidentiality by listing it as a condition in the consent form (for information sheet and consent forms for participants, see APPENDIX L and APPENDIX M). Peerreview and supervision of the thesis strictly followed ethical standards for the study.

Although free and informed consent was intended by the procedure in the study, an ethical issue may have arisen from the colleague-colleague relationship between the researcher and the participants. This may have either discouraged academics from participating (because of the change from colleague-colleague to researcherparticipant relationship where academics' actions were observed and questioned) or made some academics feel uncomfortable about withholding consent to participate. Therefore, it was necessary to make sure that the participants felt confident that participation in the study would not make them vulnerable to any harm by confirming 
the research use of their confidential information. During the time of the study, I was not in charge of quality assurance and assessment (my additional duty) within the same departmental unit; therefore, participating in my study and giving their information did not affect the academics' teaching appraisal, tenures, and promotion. The participants were also notified that their participation in the study was voluntary and that they had the freedom of choice to withdraw from the study any time before analysis. The interviews could bring rich data but also ethical challenges such as embarrassment or stress. Therefore, the researcher stayed neutral by creating a non-judgmental atmosphere, establishing rapport, and showing respect. In addition, it is also necessary to "balance the value of a potential response against the potential distress for the respondent" (Patton, 2002, p. 415). Participants were guaranteed that it was safe not to participate in the study. For example, one participant agreed to participate but withdrew because the person was not happy about being videoed; and this person felt free to withdraw.

In conclusion, for this study to be carried out properly without ethically violating human subjects, it was essential to strictly follow ethical standards. The researcher had to guarantee the accuracy and clarity of the research purpose, informed consent, researcher-participant reciprocity, confidentiality, data access, advice, and data collection boundaries.

\subsection{Conclusion}

With the research objectives and questions, and the theoretical framework, this chapter has described the selection of the constructivist paradigm and the qualitative research methodology. It has also described the design of this study as a case study. The data were collected through a semester in the implementation of TT-SET augmented with POT to investigate the intervention as a process for academic professional development. The research procedure was described with purposive sampling of participants, data collection methods, and data analysis. There was an explanation of necessary steps taken to ensure the ethics and trustworthiness of the study. 



\section{Chapter 5. Academics' Experiences of TT-SET Augmented with POT}

\subsection{Introduction}

This section provides an overall picture of participants' experience of TT-SET augmented with POT, including academics' perceptions of the intervention process and its perceived impacts on their pedagogical reasoning. Cluster analysis is used to group participants according to similar experience and perceptions of the value of the intervention in teaching improvement as described in Table 5.1.

Table 5.1. Clusters of participants

\begin{tabular}{|l|l|l|l|}
\hline \multirow{2}{*}{ Groups } & \multicolumn{2}{c|}{ Perceived value of the intervention } \\
\cline { 2 - 4 } & \multicolumn{1}{|c|}{ TT-SET } & \multicolumn{2}{c|}{ POT } \\
\cline { 3 - 4 } & & Peer feedback & $\begin{array}{c}\text { Observation of peer } \\
\text { teaching }\end{array}$ \\
\hline $\begin{array}{l}\text { Always highly beneficial: En } \\
\text { and Dang }\end{array}$ & useful & useful \\
\hline $\begin{array}{l}\text { Mostly beneficial: Dieu, } \\
\text { Hang, and Khoa }\end{array}$ & $\begin{array}{l}\text { useful but requiring } \\
\text { reconsideration }\end{array}$ & useful & useful \\
\hline $\begin{array}{l}\text { Some beneficial aspects: } \\
\text { Cuc, Giang, Nga }\end{array}$ & useful & $\begin{array}{l}\text { not very useful/ } \\
\text { not critical }\end{array}$ & $\begin{array}{l}\text { less skilled peer, } \\
\text { useful for reflection }\end{array}$ \\
\hline $\begin{array}{l}\text { Having both positive and } \\
\text { negative impact: Binh and } \\
\text { Phung }\end{array}$ & useful & $\begin{array}{l}\text { useful but } \\
\text { discouraging }\end{array}$ & useful \\
\hline Not beneficial: An & not useful & not trustful & not useful \\
\hline
\end{tabular}

The story of each participant was drawn from the varied data gathered through multiple data collection methods: interviews, observation protocols (pre-observation form, peer observer's feedback form, and academic's reflection report form), video-recordings, research journals, and TT-SETs. These stories follow a similar format, with possible variations based on the information gathered for each participant. The stories provide clues to gain insights into the academics' pedagogical reasoning and to consider the factors that may have enabled or hindered their use of TT-SET and POT for reflection. Each story starts with each group's overview of experience, teaching beliefs, aspirations, and perceptions of the value of the intervention programme. Then, following these are its perceived impacts on each academic's knowledge, actions, and other outcomes. Finally, each academic's story is summarised in a logic model. 
The logic model, which is based on the theoretical framework (see Figure 3.1) and several parts of which are adapted from Starkey (2010a), was developed during the analysis to summarise individual participants' experience of the intervention. It was used to provide an overview of how the intervention worked for an individual, with reference to the theoretical framework. Some elements (namely teaching aspirations, teaching beliefs, experience, enablers and barriers) were added because this logic model summarises the outcomes of the intervention, as described in Figure 5.1.

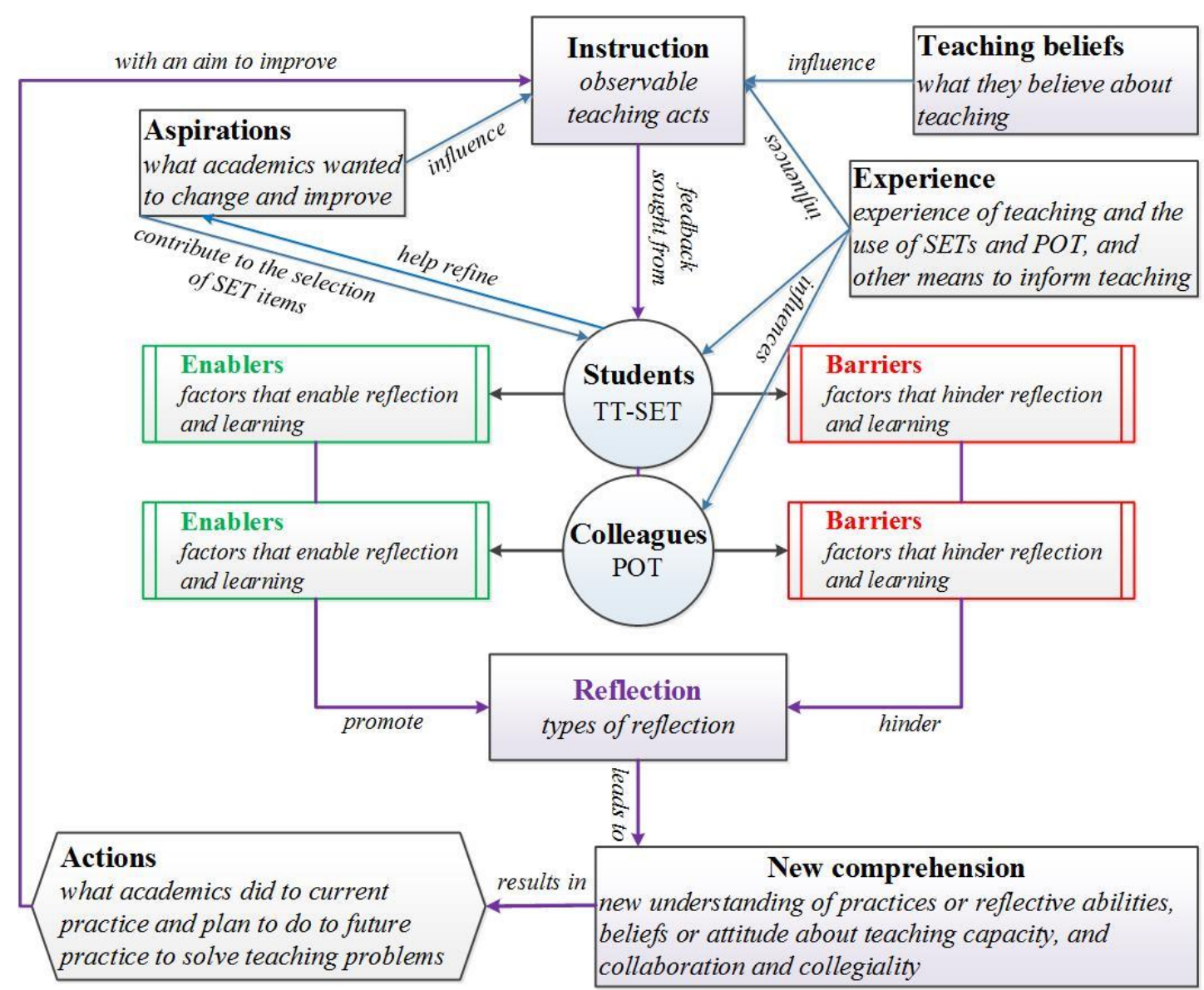

Figure 5.1: Summary logic model for each academic

The model starts with instruction (the observable teaching acts) which is affected by academics' aspirations, beliefs, and experience. Academics' aspirations contribute to academics' selection of SET items and are refined through their response to students' perceptions of their practice from early-term TT-SET. Teaching aspirations are included because they help show whether academics made changes. Teaching beliefs relate to the way academics transfer content knowledge into teachable forms (as presented in the framework). That academics had at least three years' experience of teaching and of using SET and teaching observation (in some cases for supervisory 
purposes) could have contributed to their way of teaching and perception of the intervention. Students and colleague lenses are presented in the logic model as in the theoretical framework but followed by enablers and barriers that may promote or hinder reflection respectively. Reflection, as an outcome of using the lenses, is displayed as leading to new comprehensions. Then, the ultimate outcome of the intervention is academics' action to solve problems of current or future practice. Other outcomes such as changes in beliefs or attitudes about teaching capacity, collaboration and collegiality are also included.

\subsection{Perceptions and perceived impact}

This sections looks at academics' perception of the intervention and its perceived impacts. It starts with academics' teaching beliefs and aspirations. It is noted that academics identified their aspirations from perceived teaching problems from TTSET. Then, academics' perception of the intervention is presented and followed by its impact on academics' pedagogical reasoning.

\subsubsection{En and Dang}

En had 11 years' teaching experience, and Dang has been in her career for three years. Both academics were familiar with collecting student feedback (e.g., SETs, direct talks, or observation of student attitude) to inform teaching. They had a desire to improve teaching. They had a similar view of TT-SET and POT because they perceived the two lenses as useful for their reflection.

\subsubsection{Teaching beliefs and aspirations}

En expressed her pedagogical reasoning surrounding exploiting lesson content to enhance learning interest. En defined an effective academic as having dedication and empathy with student needs that would ensure creative teaching delivery and interactions with students. She said: "An effective academic has the following qualities: creative, dedicated, empathetic for student needs, enthusiastic or patient, and caring" (En-INT(interview)).

After collecting the early-term TT-SET, En set up a plan to improve teaching on her pre-observation form as described in Table 5.2. 
Table 5.2: En's aspirations and intended strategies

\begin{tabular}{|c|c|c|}
\hline Perceived teaching problems & Aspirations & Intended strategies \\
\hline $\begin{array}{l}\text { - students needed more training on } \\
\text { listening and speaking skills } \\
\text { including pronunciation } \\
\text { correction } \\
\text { - needed to use game in classroom } \\
\text { activities }\end{array}$ & $\begin{array}{l}\text { - improve teaching methods in } \\
\text { general } \\
\text { - improve enthusiasm, prepare } \\
\text { varied knowledge and activities } \\
\text { for lesson presentation }\end{array}$ & $\begin{array}{l}\text { observing; regrouping } \\
\text { students to facilitate } \\
\text { communicative } \\
\text { activities in pairs or } \\
\text { groups }\end{array}$ \\
\hline $\begin{array}{l}\text { - needed to increase the level of } \\
\text { lessons } \\
\text { - needed to use Vietnamese in } \\
\text { explaining complex ideas }\end{array}$ & $\begin{array}{l}\text { prepare lesson content appropriate } \\
\text { for students' learning capacity }\end{array}$ & $\begin{array}{l}\text { showing rapport to } \\
\text { help individual } \\
\text { students }\end{array}$ \\
\hline $\begin{array}{l}\text { - students needed regular lesson } \\
\text { revision } \\
\text { - needed to give bonus grades }\end{array}$ & improve other aspects of teaching & $\begin{array}{l}\text { using "reward and } \\
\text { punishment" to engage } \\
\text { students in learning }\end{array}$ \\
\hline
\end{tabular}

Dang indicated that her beliefs about teaching effectiveness, teacher-student relationship, and communicative activities had affected her teaching aspirations. According to Dang, teaching effectiveness would be achieved through rapport with students and ongoing effort to learn about teaching. She also believed that establishing good teacher-student relationships would allow deeper understanding of student needs, and simple motivating communicative activities would promote learners' practice. Dang stated: “An academic can be students' sister, friend, or instructor. A good academic will continuously learn to teach and invest time in preparing lessons" (Dang-DEB (debriefing)).

After collecting the early-term TT-SET, Dang set up a plan to improve teaching as described in Table 5.3.

Table 5.3: Dang's aspirations and intended strategies

\begin{tabular}{|l|l|l|}
\hline Perceived teaching problems & \multicolumn{1}{|c|}{ Aspirations } & \multicolumn{1}{c|}{ Intended strategies } \\
\hline - needed to provide students & - give clear presentation of & organising group/pair-work so \\
with more materials & ideas and information & that students can share \\
- needed more group-work & - deliver lesson at an & knowledge and ideas for speaking \\
activities & appropriate level for & and do not fear of losing face \\
- students needed more & students & building students' confidence to \\
training on speaking and & - use more visual teaching \\
listening skills & aids (e.g., video clips) & facilitate interactions) \\
\cline { 2 - 3 } - needed to play English songs \\
for listening activities & $\begin{array}{l}\text { give more individual care } \\
\text { for students' learning and } \\
\text { assignment completion }\end{array}$ & $\begin{array}{l}\text { - observing students' interactions } \\
\text { and giving constructive } \\
\text { feedback and constant } \\
\text { individual care }\end{array}$ \\
& & $\begin{array}{l}\text { consolidating knowledge and } \\
\text { checking students' learning and } \\
\text { assignments }\end{array}$ \\
\hline
\end{tabular}




\subsubsection{Perceived values: always highly beneficial}

Dang and En believed that the TT-SET augmented with POT was valuable for informing teaching. They regarded SET as a lens to make changes to teaching. En said: "In general, SET is useful for improving teaching" (En-DEB). The academics also regarded peer feedback as being useful and reliable because of the appropriateness and good teaching ideas it brought. As noted by Dang: "Khoa's feedback was accurate and appropriate" (Dang-DEB). They valued the opportunity to observe their peer teach because they could learn good teaching techniques and self-evaluate their practice. As En said:

We can find in others something worth learning. Observing a colleague teach, we may see some aspects more interesting than ours. We may have a well-prepared lesson plan, but our colleagues may have more motivating activities. When a colleague observes our teaching, we can learn from his/her feedback. (En — INT)

Both En and Dang felt comfortable in collaborating with their peer. A sense of collegiality was felt. En said: "I felt closer to Dieu" (En- INT).

\subsubsection{Impact on pedagogical reasoning}

The intervention had positive impacts on En's and Dang's pedagogical reasoning, supporting their actions to improve their teaching.

\section{En}

The data show that En reflected on teaching and gained new comprehension. After receiving peer feedback and watching her peer teach, En realised the importance of using contexts in introducing new vocabulary instead of teaching new words in isolation. She said she learned a new strategy from Dieu:

I often present new words with explanation of their meaning. Yet, after POT, Dieu told me to ask students to find out new words in reading passages or build sentences with those words. I think it is a good idea because they could learn new words from contexts. (En-INT)

The learning led to her action for changes and future plans. En revised her teaching beliefs by emphasising the use of context in language teaching. For example, after the second POT, En started to teach vocabulary in context. The third video-recording 
illustrates the change whereby she elicited key words from the reading passage for students to guess their meanings and asked them to build sentences with these words (En-VID3 (video-recording 3)). However, the change did not yield higher student ratings (see Appendix N.1). The researcher's review also indicates that the change did not make a big difference. One of the reasons is that she planned to facilitate communicative activities in pairs or groups, but her instruction was not clearly explained to students and interactions between students were not much intensified. En also set up a plan for future changes and collection of student feedback for reflection:

I will organise more varied activities and give individual care and more suitable exercises for students at different levels. I will group the good ones and those left behind for mutual support. Then I will collect SET to know more about their opinions to improve teaching. (En-INT)

Engaging in the intervention has brought her positive attitudes. En increased in confidence throughout the process of POT because she felt that she had more credibility in teaching:

When I made changes to practice after peer feedback, students' attitudes turned positive. My teaching was improved, so they showed greater admiration for me and attended class regularly and on time. (En-INT)

En also indicated a possibility of ongoing collaborative learning with colleagues: "POT? I will participate if I have a chance" (En-INT).

In summary, with more than 11 years' teaching experience, En considered TT-SET and peer's feedback important for reflecting and improving teaching. Thanks to TTSET, she could make adjustment to teaching. Observing the peer's teaching and receiving her peer's feedback could give her some good ideas about teaching. The TTSET augmented with POT facilitated her reflection and new understanding of her practice, which appeared to trigger her action for changes. En felt confident in her teaching after the changes. She wanted to continue collaborating with her peer. She perceived that both her peer and she were open-minded and trustful, so their relationship was better. She had a positive experience of the intervention. The impact of the TT-SET augmented with POT on En's pedagogical reasoning is summarised in Figure 5.2. 


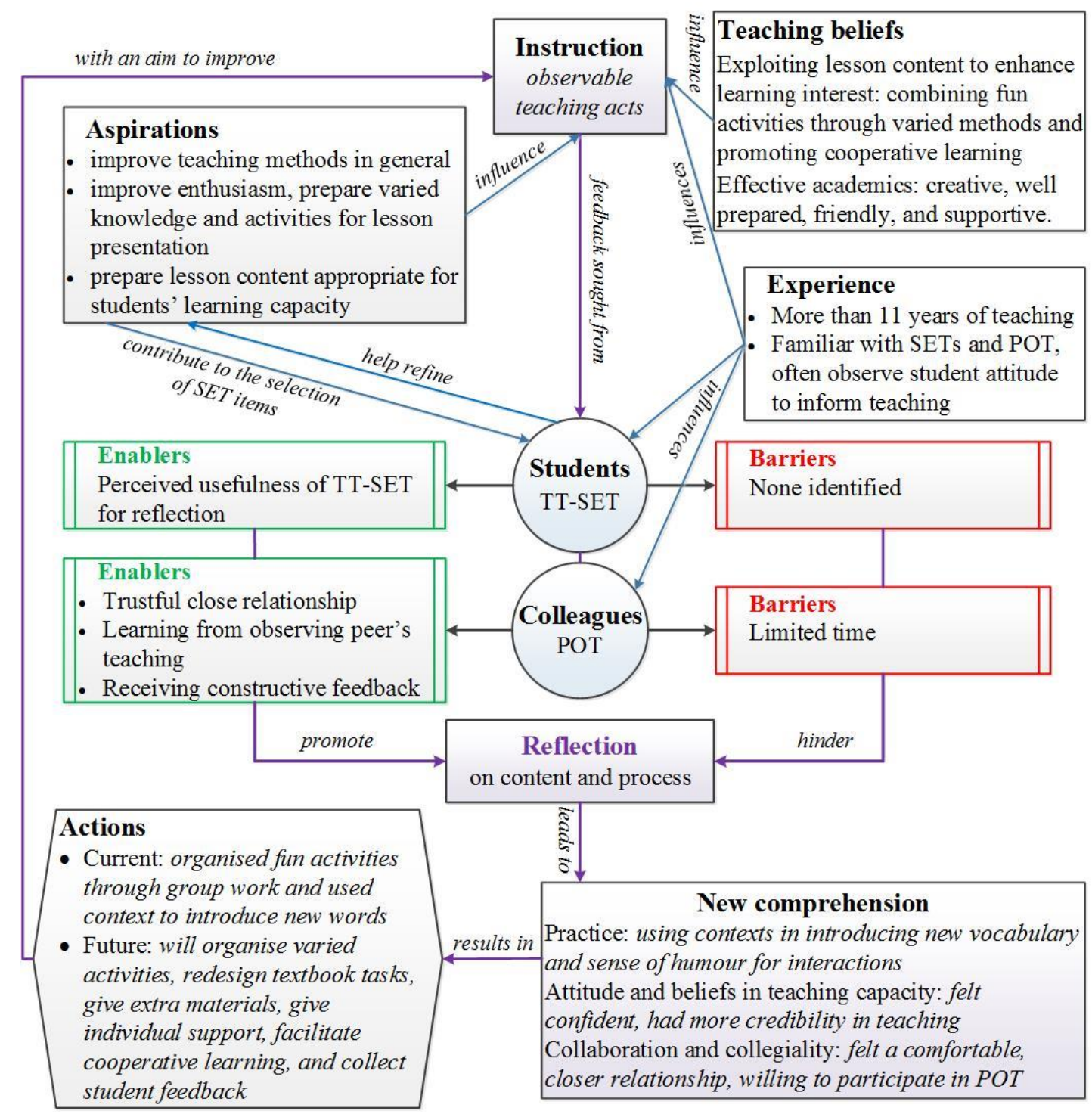

Figure 5.2: Summary logic model for En

\section{Dang}

The intervention process contributed to forming Dang's new comprehension in teaching repertoire: the importance of creating an interesting learning atmosphere. She wrote: "I learned from Khoa, using sense of humour, video clips, and games to review lessons. Khoa gave me new sources for materials, exercises, and video clips to support teaching" (Dang-TRR2 (teacher's reflection report 2)).

The new understanding of practice has shaped Dang's action to change her teaching practice. She made an adjustment to her practice. She said: "My way of teaching still emphasised students' classroom communicative activities for practice in groups, but I created a more exciting atmosphere" (Dang-INT). Dang realised that students were more interested when learning with fun activities. The third video provided evidence 
for the change: Dang used a game to help students consolidate what they had learned; the atmosphere improved, and the classroom was more exciting when students were more engaged (Dang-VID3). End-of-term ratings were high and remained unchanged (see Appendix N.2). The researcher's review indicates that Dang was making changes and her practice became livelier with more audio-visual aids. Further to the change during the intervention, Dang intended to make changes for the next semesters:

To stimulate learners' interest, I will design a variety of exercises on vocabulary, pronunciation, and grammar, which are done by groups. I will supply video clips besides pictures and guiding questions for lead-ins. Knowledge transfer will be connected to reality. (Dang-TRR2)

Dang also emphasised the necessity of continually enhancing receptive (listening) and productive (speaking and intonation) skills for students besides stimulating their learning interest. She said, "I will enhance students' listening skill and intonation" (Dang-INT).

By reconfirming the value of peer feedback on her reflection and improvement, Dang felt more confident in her teaching ability because she was able to resolve teaching problems and achieved better practice:

I learned a lot from Khoa, my teaching in this term could not have been good without POT. She showed me some aspects of which I was unaware. I changed my intonation to attract learners and created an interesting atmosphere. I learned a good way to review lessons. (Dang-INT)

Dang also indicated her enhanced collaboration with her peer: "We often share teaching ideas and materials. I like such useful activities" (Dang-INT).

In summary, Dang has been in the career for more than 3 years. After the intervention, she was aware of the importance of student and peer lenses for reflection. The academic considered POT as a good chance to learn from peer's feedback and teaching and identify her weaknesses of which she has been unaware. The intervention had positive impact on her reflection and modifying her belief (i.e. she still uses communicative approach but thought that more interesting lesson presentations (e.g., with fun activities) could engage students in learning). Responding to TT-SET and POT, Dang made some changes such as use of intonation, more video clips, and games. Her performance was rated high by students. She became confident in teaching and 
wanted to share teaching ideas with and to be observed by the peer. The impact of the TT-SET augmented with POT on Dang's pedagogical reasoning is summarised in Figure 5.3.

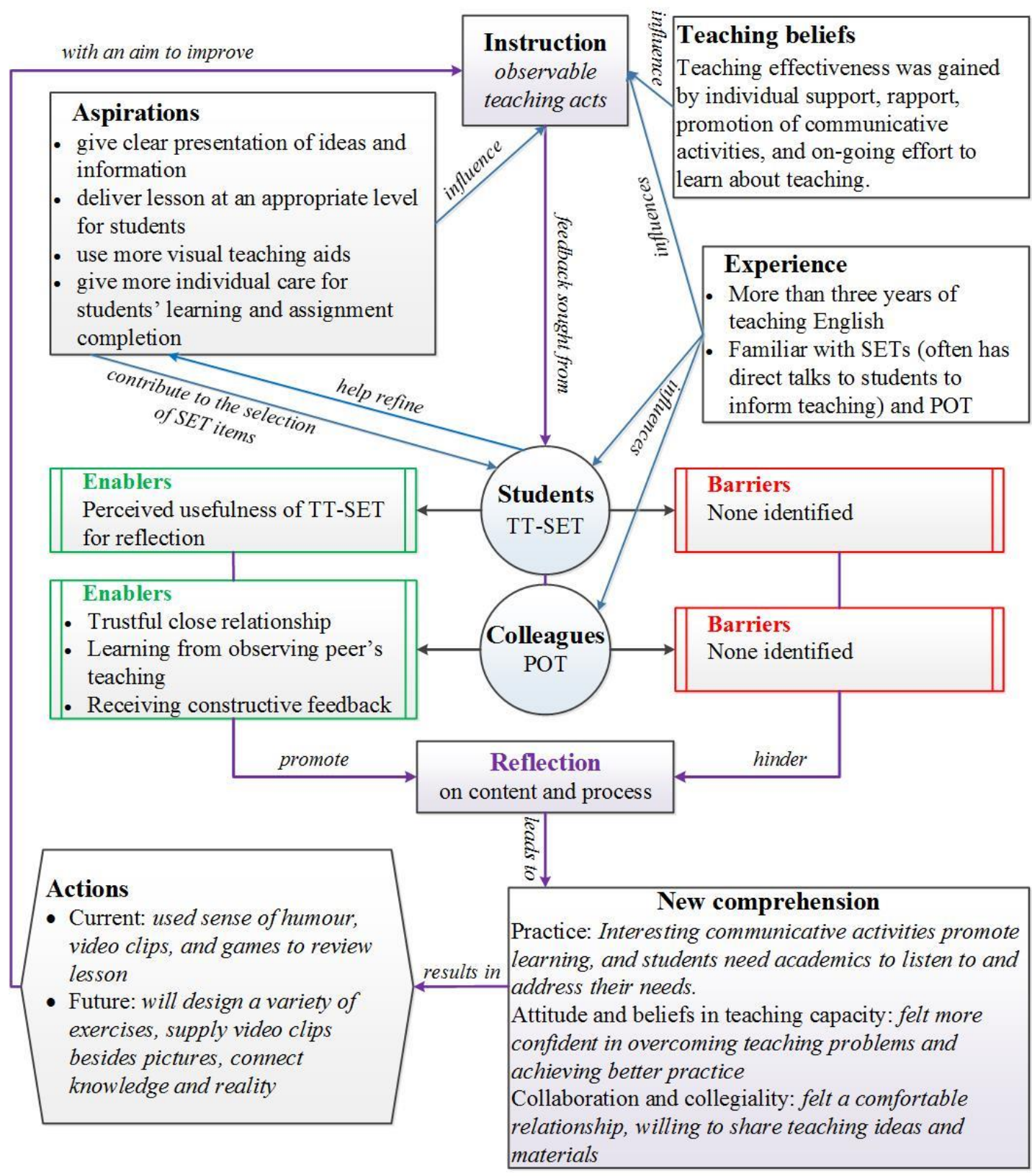

Figure 5.3: Summary logic model for Dang

\subsubsection{Summary}

Both academics valued the intervention process and exercised reflection. Their learning resulted from observing their peer's teaching and engaging in dialogue after POT1. Their reflection basically focused on the process of teaching (i.e., how to deliver lessons effectively and to attract students' attention). Although their TT-SET 
results did not show significantly higher ratings at the end of the course, from the researcher's lens they did take action for change. The impact of their reflection is that they gained confidence in teaching and felt motivated to go on making changes.

\subsubsection{Dieu, Hang, and Khoa}

Dieu had about 13 years' teaching experience; Hang has spent five years in teaching; and Khoa has been in her career for six years. The three academics were familiar with collecting student feedback (e.g., SETs, direct conversations, or observation of student attitude) to inform teaching. They thought it is important to engage in an ongoing process of learning and improving teaching. They had similar perceptions of TT-SET and POT because they perceived the two lenses as useful for their reflection, but student feedback needed critique before use.

\subsubsection{Teaching beliefs and aspirations}

Dieu believed that experience and pedagogical content knowledge played an important role in the effectiveness of academics. Besides professional knowledge, academics should have a wide range of knowledge to facilitate learning in a variety of topics:

An effective academic accumulates experience and has good pedagogical reasoning: methodology and content knowledge. Besides professional background, an academic should have knowledge of other areas in society because he or she has to dig into a variety of topics before he/she could be a facilitator. (Dieu-DEB)

Dieu also emphasised the necessity of stimulating learning interest through innovative teaching practice to teach effectively:

In general, I want to stimulate students' interest. I want to make my teaching interesting, which can help students learn better. In language teaching, if we follow 'a worn path', our performance will be boring. (Dieu-DEB)

Based on the result of the early-term TT-SET, Dieu set up a plan to improve teaching as depicted in Table 5.4: 
Table 5.4: Dieu's aspirations and intended strategies

\begin{tabular}{|l|l|l|}
\hline Perceived teaching problems & \multicolumn{1}{|c|}{ Aspirations } & \multicolumn{1}{c|}{ Intended strategies } \\
\hline $\begin{array}{l}\text { - needed a lively way of } \\
\text { organising classroom }\end{array}$ & $\begin{array}{l}\text { make more } \\
\text { interesting } \\
\text { presentation to } \\
\text { needed to stimulate students' } \\
\text { learning interest } \\
\text { stimulate } \\
\text { needed clearer way of } \\
\text { presentation for note-taking }\end{array}$ & $\begin{array}{l}\text { giving a variety of tasks to encourage } \\
\text { students' enthusiastic involvement in giving } \\
\text { opinion and taking part in other activities }\end{array}$ \\
\hline $\begin{array}{l}\text { needed to encourage students' } \\
\text { critical thinking about the } \\
\text { subject }\end{array}$ & $\begin{array}{l}\text { encourage } \\
\text { students' } \\
\text { critical } \\
\text { thinking }\end{array}$ & $\begin{array}{l}\text { - facilitating students' independent thinking } \\
\text { and defending their ideas from listening to } \\
\text { or absorbing academic's information, } \\
\text { feedback and comments } \\
\text { organising group-work and pair-work so } \\
\text { that students can share knowledge and } \\
\text { support one another for creativity }\end{array}$ \\
\hline
\end{tabular}

Hang perceived that teaching effectiveness was measured by learners' interest, learners' perceived importance of the subject, and learning outcome:

An effective academic can stimulate students' interest in learning and bring improved learning outcomes. It is also necessary to make students realise the importance of the subject in their future career. (Hang-DEB)

Hang emphasised the necessity of adapting teaching to the classroom contexts and being selective in knowledge transfer to gain teaching effectiveness:

If a class is crowded and weak, I will select what to teach. For example, I only teach pronunciation and vocabulary and ignore grammar review to save time for communicative activities... based on students' level. I transfer more profound knowledge to good students and basic knowledge to weak students. (HangDEB)

Hang believed that teacher-student mutual understanding would promote individual learning, and academics' research or experience would improve their teaching and connect content knowledge with real-life:

My belief in teaching is that it is imperative to establish teacher-student mutual understanding. In other words, academics have to show rapport with students so that every student gets help. Academics also need to do research on teaching to improve students' learning... Academics' real-life experience that is closely related to lessons can interest students. (Hang-INT) 
Combining these teaching beliefs and aspirations with the results of the early-term TTSET, Hang built her own strategies to improve teaching for the course as summarised in Table 5.5:

Table 5.5: Hang's aspirations and intended strategies

\begin{tabular}{|c|c|c|}
\hline $\begin{array}{c}\text { Perceived teaching } \\
\text { problems }\end{array}$ & Aspirations & Intended strategies \\
\hline \multirow{2}{*}{$\begin{array}{l}\text { needed an } \\
\text { environment for } \\
\text { students' } \\
\text { communication in } \\
\text { English (i.e., creating } \\
\text { chances to practice } \\
\text { listening, speaking, } \\
\text { reading, and writing) }\end{array}$} & $\begin{array}{l}\text { improve strategies to } \\
\text { promote students' } \\
\text { engagement in learning }\end{array}$ & $\begin{array}{l}\text { - creating an environment for using English } \\
\text { all the time in the classroom and saving } \\
\text { time students' communicative activities } \\
\text { - requiring students to prepare for lessons, } \\
\text { e.g., to do exercises and find useful songs } \\
\text { and games for communication in English }\end{array}$ \\
\hline & $\begin{array}{l}\text { improve strategies to } \\
\text { make students more } \\
\text { active and more } \\
\text { confident and motivate } \\
\text { students to share ideas }\end{array}$ & $\begin{array}{l}\text { encouraging pair-work and group-work to } \\
\text { build students' confidence and share ideas }\end{array}$ \\
\hline
\end{tabular}

Khoa made explicit her beliefs about an effective academic:

An effective academic can make students understand the necessity of the subject for their future career and qualify them with good premises for self-study. Sometimes they like to learn something 'practical' and dislike something 'theoretical'. They do not see the 'theoretical' as interesting because of low rationality. Saying this I mean, I will teach them 'how to fish,' not give them 'fish.' (Khoa-DEB)

Khoa adjusted her teaching practice based on students' attitudes and perceived levels of learning achievement. She increased the practicality of subject knowledge by using real-life stories to teach language:

To improve teaching, I often make changes based on students' psychological state and levels. Then, I consider the subject matter I am teaching and stimulate learners' interest by telling them real-life subject-related stories. I embed them to lesson presentation, which makes students think what they learn is practical. For example, if I teach business English, I will look for a real business story from friends or on the internet. I can see that students are more interested in learning. (Khoa-DEB)

Based on student needs found in the early-term TT-SET, Khoa set up a plan to improve teaching as listed in her pre-observation form and is combined with her aspirations in Table 5.6. 
Table 5.6: Khoa's aspirations and intended strategies

\begin{tabular}{|c|c|c|}
\hline Perceived teaching problems & Aspirations & Intended strategies \\
\hline $\begin{array}{l}\text { - needed to provide students } \\
\text { with more materials, } \\
\text { - increased more practice for } \\
\text { listening and speaking skills }\end{array}$ & $\begin{array}{l}\text { - change teaching } \\
\text { organisation through } \\
\text { varied activities } \\
\text { - make lesson presentations } \\
\text { logical and interesting }\end{array}$ & $\begin{array}{l}\text { - transferring practical } \\
\text { knowledge through humorous } \\
\text { and interesting presentations } \\
\text { - reviewing lessons more often }\end{array}$ \\
\hline $\begin{array}{l}\text { needed to organise fun } \\
\text { activities such as games and } \\
\text { creating competition among } \\
\text { learning groups }\end{array}$ & $\begin{array}{l}\text { - organise more games and } \\
\text { sense of humour useful for } \\
\text { learning } \\
\text { - promote students' } \\
\text { engagement in sharing } \\
\text { idea and knowledge }\end{array}$ & $\begin{array}{l}\text { - managing the class in groups, } \\
\text { informing course requirements } \\
\text { to create a comfortable learning } \\
\text { atmosphere } \\
\text { - planning fun games for learning }\end{array}$ \\
\hline
\end{tabular}

\subsubsection{Perceived values: mostly beneficial}

Dieu, Hang, and Khoa believed that TT-SET would be valuable if it was sound. Thus, they critically examined whether TT-SET reflected their teaching because TT-SETs may be less reliable as a result of students' indifference and distraction:

Many students would like me to be well-prepared for the lesson and make methodological changes when necessary. Others just gave their feedback without careful thought. Some others did not have any ideas. (Dieu-INT)

Students lacked wholeheartedness in giving feedback. They did not tell the truth, so SET reliability is just $50 \%$. (Khoa-INT)

Consequently, they used it critically, triangulating the information with other sources. For example, Khoa wrote: "Many students responded to the TT-SET questionnaire without care, so the information collected should be complemented with talks or interviews" (Khoa-TRR1). Yet, she said:

TT-SET is useful in some way. I compared it with my observation and students' responses to my questions during direct talks; I could draw out useful ideas... My observations and talks to get feedback are more accurate than a SET questionnaire. (Khoa-INT)

Good feedback could promote learning about teaching. The three academics believed their peer's feedback was useful for practical pedagogical advice. For example, Dieu appreciated her peer's feedback: "I see that peer feedback is really useful. My peers gave many interesting ideas about teaching" (Dieu-INT).

These academics valued the observation of their peer's teaching because they could learn new ideas. For example, Hang said: "I felt bored to teach in isolation. However, 
observing a peer colleague teach was useful and interesting because it brought me new teaching ideas" (Hang-INT). Khoa wrote in her observer's report for her peer: "I like the way you [Dang] showed tremendous care to individual students and used real-life situations to engage students in using English" (Dang-POF1 (peer observer's feedback form 1)).

\subsubsection{Impact on pedagogical reasoning}

The intervention created a positive experience for Dieu, Hang, and Khoa, assisting them in learning and facilitating action to improve their teaching.

\section{Dieu}

Dieu enhanced reflection and gained new comprehension after the intervention process. She realised that effective teaching could be promoted through task-based learning with group or pair interactions, on-the-spot application of knowledge, and lesson-and-reality connection. She explained why she regarded task-based learning as an appropriate strategy:

Task-based learning is used to encourage students to share ideas, to apply what they learned in communication, and to help one another. (Dieu-INT)

Dieu's new comprehension may be seen as resulting from premise reflection. She realised that making ongoing changes and critically examining if and why they were effective was as a way to improve practice. She said: "After getting feedback [from students and peer], I realised reflection is important for the changes" (Dieu-INT). Dieu's understanding guided her changes and future goals. To stimulate students' interest in learning and encourage them to think critically about the subject, she focused on task-based language learning to enhance students' interaction. The changes Dieu made resulted from her reflection after the first POT. As she stated:

My personal teaching philosophy is to try different ways to stimulate learners' interest and evaluate students' understanding of lessons through facilitating application of what they learned in conversations and writings. (Dieu-TRR1)

The video after POT showed Dieu's attempt to organise a variety of activities: groupwork games (listing words) after presenting word formation, sentence transformation exercises, writing sentences from pictures as hints. Students were actively engaged when joining in a competitive exercise; a picture was shown for them to build a passive sentence. However, her delivery was sequenced with a great deal of dead time. 
Activities to encourage critical thinking in the classroom were absent. Passive voice was introduced deductively through a formula of the sentence structure, explanation, and examples. There was much teacher-talking time, and students were quiet most of the time (Dieu-VID3). The change did not yield higher student ratings. Actually, ratings in end-of-term TT-SET for most of the items Dieu chose remained unchanged (see Appendix N.3). Students rated her way of encouraging students' discussion lower on the end-of-term TT-SET. From the researcher's review, Dieu made changes but they did not occur as Dieu intended. Her idea of task-based learning was good, but it was affected by her ineffective classroom management skills (i.e., creating much dead time). In addition, she did not have an effective way of presentation to stimulate students' critical thinking.

Consistent with what was planned earlier, Dieu indicated her intention to continue making changes to teaching (e.g., towards communicative language teaching with a focus on task-based language learning). She investigated the use of meaningful tasks with authentic language. She believed that facilitating more interactions among students through games and guiding questions would stimulate students' interest in learning:

My teaching will be directed towards communicative language teaching to help students have more interactions. I gave different tasks for different skills. I saw that students were interested in doing the tasks. For example, before a listening task I designed activities like a game or asked guiding questions to elicit their ideas about the topic. I see task-based language learning is really effective. (Dieu-INT)

Reflecting through student and colleague lenses, Dieu developed a sense of "being better" because she believed that her practice had improved. This may indicate a developed sense of self-efficacy. She was motivated to continuously improve practice and join in cooperative learning with colleagues:

Thanks to the process, my teaching practice is better. I keep in my mind that I must go on improving teaching so that others can learn from me and I can learn from others. (Dieu-INT)

In summary, although Dieu was concerned about the quality of TT-SET, she was aware of the importance of students' and peer's feedback for reflecting and improving teaching. The lenses were perceived as useful for timely changes. Observing the peer's 
teaching and sharing pedagogical ideas helped her identify her strengths and weaknesses. The intervention facilitated Dieu's reflection and new understandings, particularly reflective practice facilitating regular changes. Dieu made changes to teaching but did not create a big difference in practice, she planned action for future practice. She felt confident in teaching. Her peer relationship became better and she wanted to share teaching ideas and participating in POT with others. Dieu's experience of the intervention is summarised in Figure 5.4.

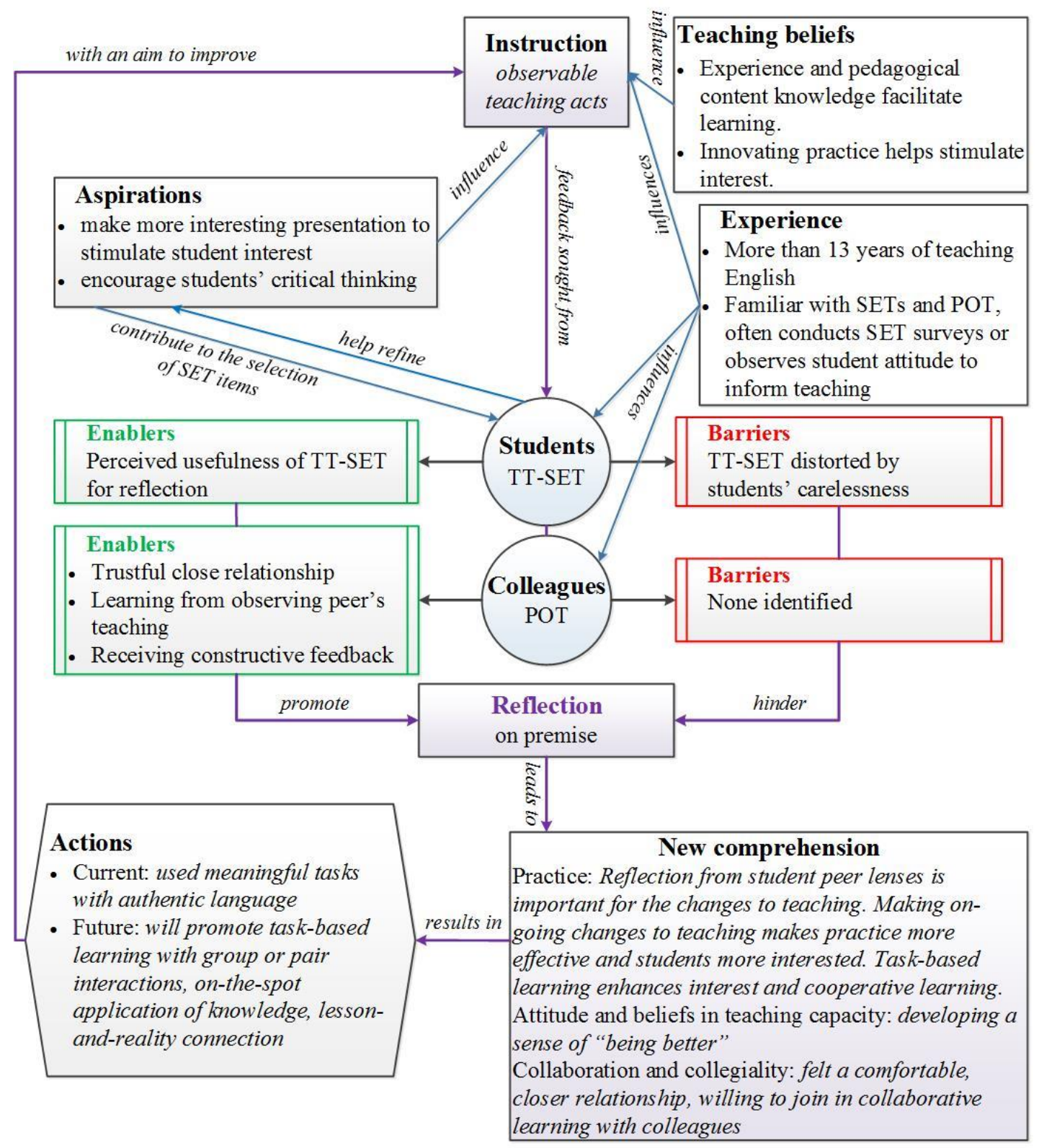

Figure 5.4: Summary logic model for Dieu 


\section{Hang}

Hang's new comprehension of practice was centred on flexibility and selectivity of lesson presentation. POTs provided Hang with experience from which she learned effective ways to prepare for teaching, techniques for clarity, supply of materials, and selection of knowledge to transfer:

I learned from Nga the attitudes, knowledge, and preparation for teaching: good preparation, clarity of presentation, and supply of more materials. So far, I have relied on the textbook, but now I am more selective in knowledge transfer. (Hang-TRR2)

Hang's understanding resulted from reflection on the process of practice. Not only gaining vicarious experience, Hang also took actions to change teaching, particularly in solving teaching problems.

Hang used student and peer lenses to resolve her problems of teaching practice. Hang reflected based on her peer's feedback and considered the issues raised by her peer: "Actually, I did not make many big changes due to a short span of time in a term, but I considered Nga's feedback and made some teaching adjustments" (Hang-INT).

Based on her peer's feedback on aspects requiring improvement after the first POT, Hang changed her teaching style and helped students with a conceptual grasp of lessons, noting that, "I was more enthusiastic and spoke English more slowly. I gave more examples for difficult words or grammatical concepts" (Hang-TRR1). Compared with previous practice through videos before and during POT, Hang's teaching after POT changed. She presented lessons more clearly. An example of this was that for a listening task she introduced vocabulary by eliciting students' ideas on their meanings and giving examples to clarify the meanings. After listening, pairs compared their answers and wrote them on the board. Hang spoke more slowly to explain new words and ideas (Hang-VID3). Ratings in TT-SETs were high and remained unchanged for most of the items, but Hang received a higher rating for her way of stimulating students' interest in learning (see Appendix N.4). From the researcher's viewpoint, Hang achieved one of her goals - engaging students in learning because her way of organising activities involved students in interactions. The intervention process not only enabled Hang to improve teaching practice, it also initiated her to think about future change. She indicated her wishes to refine her 
teaching style and use a learner-centred approach: "I will present lessons more slowly, ask students questions to establish a habit of discussion, and help them develop critical and creative thinking" (Hang-TRR2).

Hang expressed a positive attitude towards her ability. She was more confident in teaching and improved her pedagogy because she had confidence receiving feedback from an experienced peer and had opportunities to observe her teach:

POT helped me learn a lot. I got Nga's feedback on my teaching, and learned good things from observing her teaching: how she helped students explore and get involved in lessons... The feedback was useful for me because Nga is experienced and has good pedagogical knowledge. (Hang-INT)

Hang showed her willingness to learn through POT: "It is useful to learn from others, so I like to discuss teaching ideas with colleagues" (Hang-INT).

In summary, Hang has been teaching for more than 5 years. She was aware of using students as lenses for reflection despite her concern for its reliability and valued peer's feedback much more for shaping her teaching practice. The intervention positively impacted on her reflection and somehow changed her belief about the flexibility of content knowledge transfer. That is she turned from strictly following the textbook content to flexibly selecting content delivery to learners. Thanks to the response to SET and peer's feedback, Hang made some changes such as giving clearer detailed instruction and explanations and considered her speech speed, but she did not change her way of stimulating interest. Yet, she got higher end-of-term ratings for her effort in group-work activities, eliciting, clear presentation, and rapport. She became confident in teaching and wanted to share ideas about teaching via POT with colleagues who had a good relationship with her and who she felt comfortable to work with. However, she preferred POT being organized for longer time (at least two semesters) so that changes could be facilitated. The impact of the TT-SET augmented with POT on Hang's pedagogical reasoning is summarised in Figure 5.5. 




Figure 5.5: Summary logic model for Hang

\section{Khoa}

Khoa's new understanding of practice emphasised diversified teaching organisation from vicarious experience attained through watching her peer teach and receiving peer feedback. She realised the necessity of diversified teaching organisation with fun activities and appropriate class management:

To stimulate students' interest in learning, I will restructure textbook lessons with appropriate timing; use a variety of activities with games, video clips, and songs 
with relevant grammatical structures; and regroup students for individual rapport and easy management. (Khoa-TRR1)

After watching her peer use a strategy that engaged students in learning, Khoa learned that facilitating student self-study could promote learning. Khoa also thought that she would steer student thinking to prioritise the importance of content knowledge:

I realised that Dang was well prepared and promoted students' self-study. I learned that giving students time to self-study helped them better understand lessons. Thus, I will give time for students to prepare and self-study and encourage communication by all means, even forcing them to do so. (KhoaTRR2)

My teaching approach tended to prioritise learners' favourite way of learning and balance both assigned and learner-interested content (50\% and 50\% respectively). However, after POT I realised that if students were excessively offered what they like, I would fail to transfer necessary knowledge. (Khoa-INT)

Khoa explained the premise of her choice of teaching strategies. This new understanding based on reflection led to Khoa's actions to change her teaching practice. Khoa made changes regarding the balance between knowledge transfer and students' interest in that content knowledge was emphasised and more teacheroriented:

I forced students to go into my way, awakening their perceptions of the importance of the knowledge even though they do not know how useful it is. The balance is now $30 \%$ for students' interest and $70 \%$ for my interest on what should be learned. (Khoa-INT)

She also adjusted timing appropriate for students who had difficulty keeping up with the lesson and showed care for them:

I timed lessons appropriately so that weak students could keep pace with the rest. I search for funny stories on the internet to interest learners. (Khoa-INT)

Compared with before and during POT videos, the third one showed that Khoa reduced dead time and teacher-talking time. She used pictures to introduce reading passages and guessing games through PowerPoint to revise key words. She expressed a sense of humour during the class, using jokes and intonation. Some changes in teaching organisation with more group discussion were evident. Students tended to be more engaged. However, students were not instructed in the production stage where they 
could use or reproduce what they had learnt after some practice, and the focus of the lesson was unclear, such as instruction on skills or grammar points. Khoa showed support to students who could not keep pace with the lesson by moving around to offer help while they were doing exercises and discussing (Khoa-VID3). Ratings in TTSET remained unchanged for all items of feedback (see Appendix N.5). From the researcher's review, Khoa attempted to enhance practice, but she did not clearly emphasise what was intended for each teaching session. For future change, Khoa intended to apply entertaining activities with her lesson delivery to stimulate learning interest: "I will organise competitive games or play music to interest students and make them memorise lessons well" (Khoa-INT).

Khoa reported that her sense of confidence in pedagogy was developing from vicarious experience. She believed that the new teaching technique would work in enhancing students' learning:

POT has been challenging and inspiring to me, so I felt so motivated that I tried all my best and was more creative. I realised my teaching has been improved, and I was able to identify aspects of teaching which I was unaware of. I felt confident when Dang said: "You organised classroom activities logically". (Khoa-INT)

Khoa was willing to join in POT: "I often share teaching ideas and materials with Dang and like to participate in POT with her" (Khoa-INT).

In summary, Khoa has been in the career for more than 6 years. She regarded TT-SET as useful though she had some concern. Khoa considered POT as an opportunity to learn from peer's feedback and teaching and identify her weaknesses of which she has been unaware. The intervention had positive impact on her reflection and changes her belief (e.g., delivering content she thought to be useful for students' future work is better than organising fun activities students like). Responding to TT-SET and POT, Khoa made some changes such as timing lesson more appropriately and paying care to individual students, used stories to engage students in learning. The TT-SET results remained unchanged. She became confident in teaching and wanted to share teaching ideas with and to be observed by the peer. Khoa's experience of the intervention is summarised in Figure 5.6. 


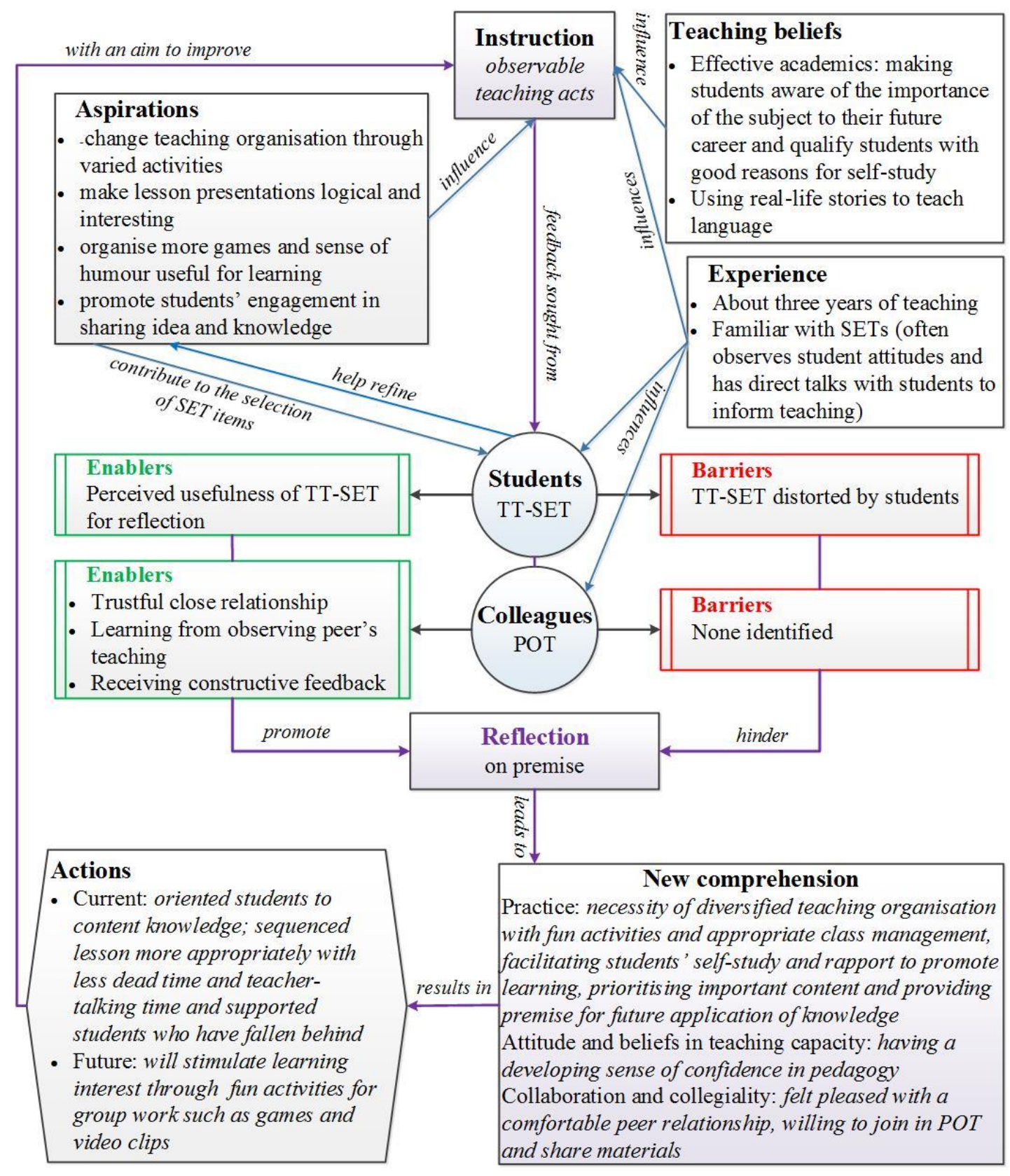

Figure 5.6: Summary logic model for Khoa

\subsubsection{Summary}

The three academics regarded student feedback as useful but used it with caution because they thought that it was distorted by other factors. The academics valued the POT experience. They learned new things from watching their peer teach and discussing teaching with their peer. While Hang reflected on the how of practice, Dieu and Khoa focused on the why of teaching; they gave their reasons why they changed their practice. All three academics made changes and intended to make changes to their future practice. They all improved their confidence in teaching. Particularly, Hang 
learned how to teach from verbal persuasion and achieved a higher rating in one aspect in addition to her vicarious experience.

\subsubsection{Cuc, Giang, and Nga}

Cuc had about eight years' teaching experience; Giang has spent 14 years in teaching; and Nga has been in her career for 13 years. The three academics were familiar with collecting student feedback (e.g., SETs, direct conversations, observation of student attitude, students' profiles, and orientation sessions) to inform teaching. They thought TT-SET was useful, but Nga thought it may be distorted on first impression. They had similar experience of POT in that their peer, who was much younger, did not give critical feedback and perform skilful teaching. They thought POT in this case may not be very beneficial to them.

\subsubsection{Teaching beliefs and aspirations}

For effective teaching, Cuc emphasised the importance of facilitating students' selfstudy and triggering the desire to learn by promoting communicative activities. She believed an effective academic should create a safe environment for students to try out new learning:

A good and effective academic should build up students' confidence in ability to study on their own. A good academic does not need to have high expertise, but has an ability to inspire students to study and help them to identify self-study ability. I let my students study and develop naturally without attention to accuracy. I would like them to be willing to speak out regardless of being right or wrong. When they join in the real world of work, they will make changes. (Cuc-DEB)

Cuc expanded her beliefs that promoting communicative activities and integrated skills could enable students to develop comprehensive language skills, which are necessary for promoting their specialised competence. According to her, it was vital to equip students with skills and content knowledge:

It is necessary to integrate all language skills [reading, writing, listening, and speaking] in one teaching session, to stimulate their interest, and to build up confidence and activeness in studying. (Cuc-TRR1)

As you know, the experience I gained while working with international relations (I have to contact many people from other countries) shows that language and 
social skills are necessary. When students are sufficiently skilled, they will better their specialised knowledge and competence. (Cuc-INT)

Considering feedback from the early-term SET, coupled with teaching aspirations and belief, Cuc designed strategies to improve teaching (Table 5.7).

Table 5.7: Cuc's aspirations and intended strategies

\begin{tabular}{|c|c|c|}
\hline Perceived teaching problems & Aspirations & Intended strategies \\
\hline $\begin{array}{l}\text { - needed to provide students with more } \\
\text { training in listening, speaking, reading, } \\
\text { and writing } \\
\text { - needed to organise games } \\
\text { - needed to provide students with extra } \\
\text { materials } \\
\text { - needed to enhance clarity of lessons with } \\
\text { practical examples } \\
\text { - needed to promote group-work activities, } \\
\text { creating communicative situations }\end{array}$ & $\begin{array}{l}\text { - promote teacher- } \\
\text { student and } \\
\text { student-student } \\
\text { interactions } \\
\text { - integrate listening, } \\
\text { speaking, reading, } \\
\text { and writing in one } \\
\text { session } \\
\text { - improve teaching } \\
\text { organisation }\end{array}$ & $\begin{array}{l}\text { - using group-work } \\
\text { discussions so that } \\
\text { students can learn from } \\
\text { one another and become } \\
\text { confident in using } \\
\text { English } \\
\text { - giving students bonus } \\
\text { grades }\end{array}$ \\
\hline $\begin{array}{l}\text { - needed to offer guidelines on learning } \\
\text { methods } \\
\text { - needed to change the method of evaluating } \\
\text { students' learning }\end{array}$ & $\begin{array}{l}\text { offer guidelines on } \\
\text { learning methods }\end{array}$ & $\begin{array}{l}\text { encouraging students to } \\
\text { communicate in English } \\
\text { without making judgment } \\
\text { on their accuracy }\end{array}$ \\
\hline
\end{tabular}

Giang regarded a sense of humour and an ability to communicate ideas and information clearly as important in teaching. Being well prepared can help develop this ability, which she believed facilitates the use of a learner-centred approach:

I think a sense of humour and an ability to communicate ideas and information are important in teaching. Whether I can teach in a lesson clearly or humorously depends on my preparation for the lesson. (Giang-DEB)

Giang indicated an aspiration to use an inductive approach: "I set tasks for students to do. Then, they themselves find out the rules. After that, I confirm the rules. I like this way" (Giang-INT).

In addition, enthusiasm and commitment with students, which can enhance students' interest in learning, contribute to the qualities of an effective academic:

Most important is being a facilitator who knows how to deliver the course book, making it unique. Students do not like reading lessons much, so my job is to encourage them to open the book, and games were a way to stimulate them to look for answers. I think my enthusiasm motivated students to learn. (Giang-INT)

Because we already had a commitment, when a session started, my students had 'automatically' prepared for their presentations. If they did not, the entire class's 
grades were deducted. You know, I maintained their willingness in learning by persuading and threatening. (Giang-DEB)

Giang also believed that giving constructive feedback to facilitate students' reflection and engagement was a way to encourage learning:

When an activity for groups was organised, the team leader was supposed to assign group members' tasks. Group members would share ideas and help each other. When the team leader could not help those left behind, I would help them. I then gave feedback without comments on accuracy and let them think. I think it is a way to encourage them to learn. (Giang-INT)

After collecting the early-term SET, Giang set up a plan to improve teaching as listed on her pre-observation form (Table 5.8).

Table 5.8: Giang's aspirations and intended strategies

\begin{tabular}{|c|c|c|}
\hline Perceived teaching problems & Aspirations & Intended strategies \\
\hline $\begin{array}{l}\text { - needed to facilitate more learning } \\
\text { organising teaching activities } \\
\text { - needed to enhance clarity of } \\
\text { communicated ideas and information } \\
\text { - needed to hold learners' interest } \\
\text { during presentations } \\
\text { - needed to deliver intellectually } \\
\text { challenging content } \\
\text { - needed to deliver lessons at an } \\
\text { appropriate level for students }\end{array}$ & $\begin{array}{l}\text { - improve ways of } \\
\text { organising teaching } \\
\text { that facilitates } \\
\text { learning } \\
\text { - enhance clarity of } \\
\text { communicated ideas } \\
\text { and information } \\
\text { - provide intellectually } \\
\text { challenging materials }\end{array}$ & $\begin{array}{l}\text { - organising group work so } \\
\text { that the students could } \\
\text { support one another } \\
\text { - facilitating students' } \\
\text { learning through organising } \\
\text { groups managed by group } \\
\text { leaders }\end{array}$ \\
\hline $\begin{array}{l}\text { needed to give constructive individual } \\
\text { feedback }\end{array}$ & $\begin{array}{l}\text { give constructive } \\
\text { individual feedback }\end{array}$ & $\begin{array}{l}\text { visiting each group during } \\
\text { their discussion to give } \\
\text { support when necessary }\end{array}$ \\
\hline
\end{tabular}

Expressing her teaching assumptions, Nga believed that teaching effectiveness was measured by students' developed ability for self-study and academics' innovative methods:

When teaching reading, it is not just to help students do exercises but show them what skills (e.g., skimming and scanning) they will develop after completing the task. As for vocabulary, academics have to pick out new words and organise appropriate practice so that students will have something in their mind. Teaching should be innovative. (Nga-INT)

Knowing that students like varied activities and fun, Nga believed that an effective academic should be creative and active: 
In general, students like interesting lessons, e.g., games, jokes, and varied activities. Something that recurs will be boring; thus, an academic should be creative and active. So, my way is that I deliver content (theory), facilitate students' practice, and organise games to 'break the ice.' (Nga-INT)

After collecting the early-term SET, Nga set up a plan to improve teaching as listed on her pre-observation form (Table 5.9).

Table 5.9: Nga's aspirations and intended strategies

\begin{tabular}{|l|l|l|}
\hline \multicolumn{1}{|c|}{ Perceived teaching problems } & \multicolumn{1}{c|}{ Aspirations } & \multicolumn{1}{c|}{ Intended strategies } \\
\hline $\begin{array}{l}\text { - needed to provide more training in students' } \\
\text { ability to make oral presentations } \\
\text { - needed to make class atmosphere more lively }\end{array}$ & $\begin{array}{l}\text { improve the } \\
\text { liveliness of the } \\
\text { atmosphere }\end{array}$ & $\begin{array}{l}\text { encouraging pair-work and } \\
\text { group-work discussions, } \\
\text { groups oral presentations, } \\
\text { interactive communication }\end{array}$ \\
\hline $\begin{array}{l}\text { needed to train students how to respond } \\
\text { appropriately to certain situations in English }\end{array}$ & $\begin{array}{l}\text { instruct students } \\
\text { with innovative } \\
\text { learning methods }\end{array}$ & $\begin{array}{l}\text { setting work to promote } \\
\text { student learning (for future } \\
\text { work life) and confidence }\end{array}$ \\
\hline
\end{tabular}

\subsubsection{Perceived values: some beneficial aspects}

Though the intervention was perceived as useful, it had limitations. First, Cuc, Giang, and Nga regarded TT-SET as valuable for informing teaching:

You have known me for a long time! I respect students and highly value their feedback. I see my students as lenses to self-reflect and make changes... In my view, the quality of SET depends on when it is collected. Yet, in general, most students were trustful in giving feedback. (Cuc-INT)

However, Nga perceived that student feedback required critique before use:

The SET collected after three weeks of teaching just provided a temporary view of my teaching and was affected by the impression I made in the sessions. I think the end-of-term SET is more reliable. (Nga-INT)

The three academics appeared to underrate peer feedback because of beliefs that their peers were either insufficiently critical in giving feedback or unskilled:

I do not highly value the peer's feedback. For some reason, maybe the influence of oriental culture - respect to the seniors and to those with a higher status - she was reluctant to give feedback. She may have had some ideas to help me improve my teaching. (Cuc-INT) 
They regarded teaching observation as an opportunity to detect similar problems their peers faced and to reflect on their teaching. They did not see it as an opportunity to learn new strategies:

Honestly speaking, I did not learn anything when observing Hang's session.

However, I saw her weaknesses and referred to my context. (Nga-TRR1)

Nga meant that she could not learn better teaching methods from Hang, but found the chance to observe her teaching useful because it was useful evidence for her reasoning about teaching. She clarified in the interview: "Observing Hang's teaching helped me find evidence for my reasoning, e.g., to examine if a combination of communicative approach with a traditional method like grammar translation could support every student in learning" (Nga-INT).

\subsubsection{Impact on pedagogical reasoning}

\section{Cuc}

Cuc's new comprehension was centred on adjusting knowledge transfer to suit students' ability. Watching her peer teach, Cuc noticed that simplifying knowledge was an effective way to engage weak students in participation in discussion. She also valued the importance of creating a good teacher-student relationship and engaging students in basic practice:

Binh tried to simplify knowledge, i.e., to make complicated issues simple and practical in order to suit students' ability and enhance their interest in learning. Students were happy when the academic remembered their names, which strengthened their relationship. It was necessary to focus on basic practice, which helped weak students catch pace with others. (Cuc-TRR2)

During the process, although receiving only compliments from her peer, Cuc decided to change teaching practice based on student needs, her evaluation on her peer's teaching aspects that needed improving, and reviewing her teaching beliefs. Cuc promoted the strengths of her pedagogy and changed practice to suit student needs. She clarified:

Students like what I am doing. They like to study with no pressure in studying, group-work communication, lessons from academic's experience, lesson on learning methods rather than overloaded content delivery. (Cuc-INT) 
She emphasised the necessity of integrating four language skills rather than transferring content knowledge:

After getting TT-SET two weeks later, I saw that integrating four language skills in one lesson met their needs. Then, I made a change: Content knowledge was supplementary to skill development in my teaching delivery. (Cuc-INT)

Cuc had consistent teaching strategies to achieve her goals as evidenced in the videorecordings. She integrated four language skills in one class session, showing similar enthusiasm and effectiveness in organising varied activities with fast grouping and clear instruction. She maintained good teacher-student interactions (with guiding questions and eliciting) and student-student interactions (with group work). Students' engagement in these activities was improved throughout the course. Students were instructed how to self-study (e.g., how to use a dictionary) (Cuc-VID2 \& 3). Results of TT-SET were high and stable (see Appendix N.6). From the researcher's perspective, Cuc was an able academic who was willing to try new teaching ideas, and was consistent in teaching methods, showing a trajectory towards promoting her strengths. For future improvement, Cuc indicated an aspiration to build teacher-student relationships, develop students' language skills, and simplify knowledge transfer. She said: "Next term, I will consider providing students with basic knowledge and promoting their language skills" (Cuc-INT).

Cuc's teaching practice was responsive to student feedback, which led to achievement. She felt confident in teaching from mastery experience: "I never checked attendance, but students came to class. It was evidence that my instruction motivated them to learn" (Cuc-INT).

In summary, Cuc had more than 8 years' teaching experience. She regarded using student and colleague lenses as useful for reflection. The TT-SET helped inform her about teaching performance and reconfirm the effectiveness of the methods, the appropriateness of her teaching styles and theory. Observing the peer's teaching was a way of reflecting on practice. The intervention facilitated her reflection and new understandings. She made changes to teaching based on the new comprehensions. Her performance was highly valued by the peer and students. The end-of-term TT-SET produced higher ratings on the aspects of teaching she paid attention to. Cuc became confident. She was open-minded and willing to share ideas about teaching with colleagues. However, because the peer did not give feedback wholeheartedly, she 
expected to learn from other peers who were not affected by her status and compatible in experience and expertise. Cuc's experience of the intervention is summarised in Figure 5.7.

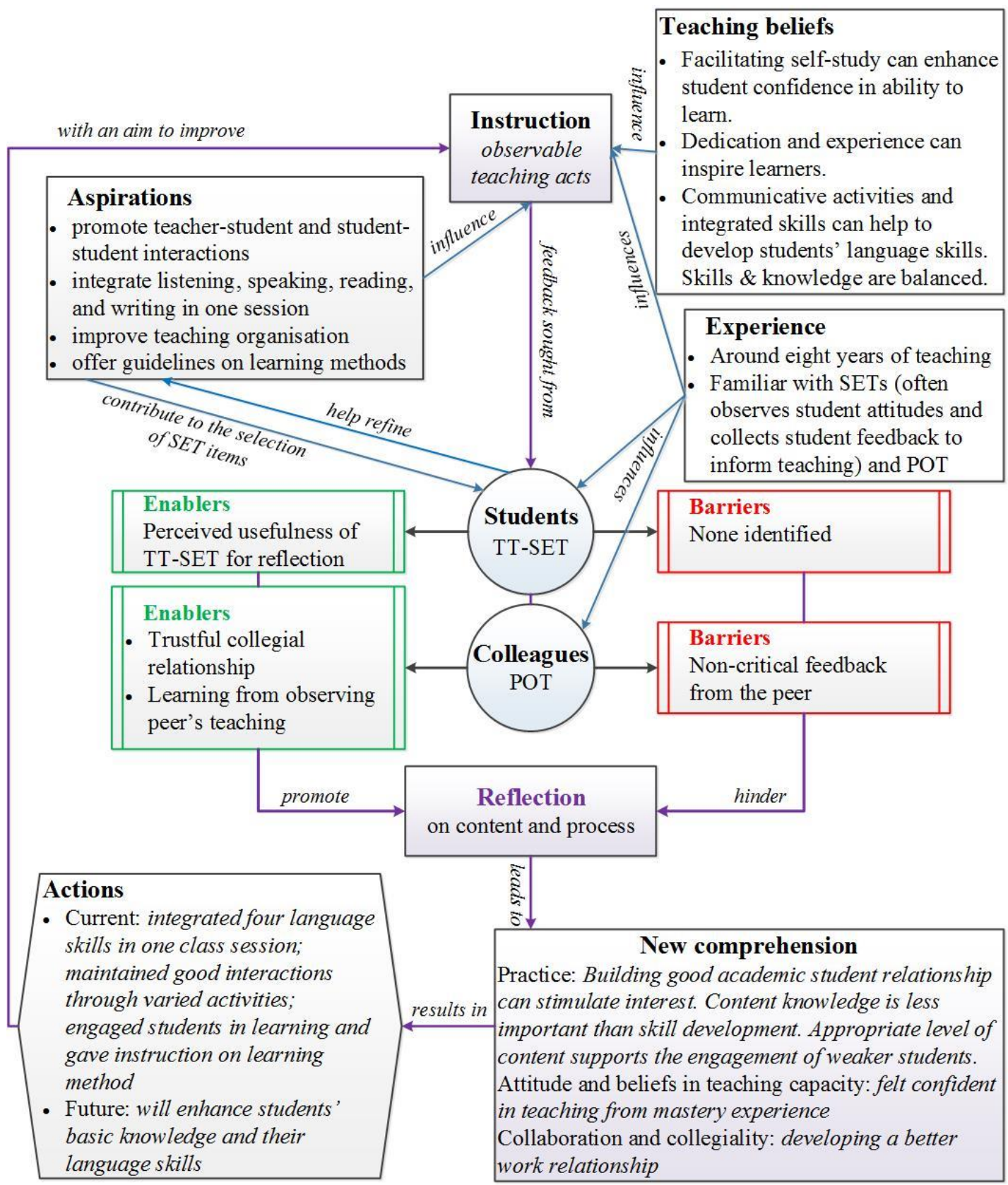

Figure 5.7: Summary logic model for Cuc

\section{Giang}

Giang's new comprehension of teaching related to teaching style. She admitted that over-excitement in organising activities sometimes resulted in her neglect of delivering lesson content knowledge at a level appropriate for those who had difficulty keeping up with the lesson: 
I agree on the idea that I was too enthusiastic when students played games that I ignored other important points. (Giang-INT)

This understanding helped her refine practice by paying attention to her style and delivery of lesson content, considering whether teaching delivery was appropriate for students:

Before, I just presented new words by giving phonetic transcription and definitions without instruction on pronunciation. I thought students could keep pace with this. After I had observed Phung guide her students to pronounce new words, I spent time instructing my students to read new words and check their pronunciation. (Giang-INT)

Giang was consistent in practice as shown in the video-recordings: organising group fun activities to stimulate student interest, making lessons clear, and giving feedback on student presentations (Giang_-VID1, 2, 3). After the first POT, Giang engaged more students in class activities. Students were asked to present group homework; then, presenters and the rest shared ideas about the presentation topic, followed by Giang's detailed constructive feedback. Giang adjusted practice and cared about students who could not keep up with the lesson by giving a detailed explanation (often in Vietnamese) of ideas (Giang-VID3). Results of end-of-term TT-SETs indicated significantly higher ratings for three items related to teaching organisation, clarity of communication, and constructive individual help (see Appendix N.7). From the researcher's review, Giang adjusted her delivery to suit students. Giang intended to improve her teaching organisation. She said: "I will diversify classroom activities to motivate learners" (Giang-INT).

Giang enhanced awareness of her practice, so positive student feedback and dialogue with the peer brought more certainty of belief in her teaching: "The process helped to confirm my reflection" (Giang-INT). This might have increased her sense of selfefficacy. Giang and Phung had a trusting peer relationship, and Giang said about her willingness for POT: "Because we trust each other, I am willing to be a teaching model if Phung wants to watch me teach" (Giang-INT).

In summary, Giang had more than 14 years' teaching experience. She considered TTSET as useful for ongoing changes to teaching practice and peer's feedback as useful for her learning. Giang thought she could not learn new teaching ideas from observing the peer because she is less skilled. The intervention had positive impact on her 
reflection and changes in her belief about content knowledge delivery. She became careful in delivering content at an appropriate level for students. Giang made changes to her teaching. She got higher end-of-term ratings for her effort in using sense of humour, diversity of group-work activities, especially games, clear presentation, and rapport. Giang became confident in her teaching and wanted to share ideas about teaching via POT with colleagues who were her friend (i.e. those who had good relationship with her). However, she would like to have an observer who was compatible with her in age and experience. In addition, she preferred POT being organized for longer time (at least two semesters). Giang's experience of the intervention process is summarised in Figure 5.8.



Figure 5.8: Summary logic model for Giang 
$N g a$

Nga's new comprehension focused on using integrated methods and caring for those who had difficulty catching up with the lesson. After watching her peer teach and reflecting on practice, Nga found it necessary to combine varied methods to suit students at different levels in the same class:

In the past, I thought a communicative approach was effective in language teaching, but now I believed it should be combined with traditional language teaching. For example, teaching grammar like articles requires very slow, careful and detailed explanations followed by interesting activities. (Nga-INT)

Revising her teaching beliefs from addressing the why of teaching, Nga identified the peer's aspects of teaching requiring improvement and figured out solutions to her own problems. She stated:

I saw that Hang did not pay attention to students' reactions and focused on her delivery. This means individual care was not considered. She might have subjectively thought that her teaching was at a level appropriate to students. She taught so fast even though there were some left behind. Observing Hang's teaching helped me refine my teaching. For example, I delivered the reading slowly and carefully, providing contexts and let students explore word meaning on their own. Then, I consolidated the issues and gave compliments to those who worked well. I think it motivated them a lot. (Nga-INT)

I involved students, even weak students, in most class activities. In fact, the class atmosphere is improved with laughs and engagement. (Nga-TRR2)

Nga gained her new comprehension that the peer lens helped her examine how her reasoning worked in other classroom setting and become more critical in reflecting on practice. She said: "By observing the peer's teaching, I know what combination of my approaches works. Then, I became more critical in analysing, for example, if these strategies work for students" (Nga-INT).

Nga was consistent in her method. Lessons were logically presented with pair work or group work discussions and stimulus response. Starting lessons by reviewing previous lessons, Nga organised fun activities to build a comfortable atmosphere. After students had presented ideas, she offered correct answers and compliments for good responses. Nga gave clear instructions, detailed explanations, and consolidations for learning issues (Nga-VID1, 2, 3). In the third video, she refined her method to help students 
learn, e.g., translating the reading passage (Nga-VID3). Results of end-of-term TTSET were high and remained unchanged (see Appendix N.8). From the researcher's review, Nga refined practice and was consistent in teaching methods she aimed to apply.

Throughout the process, Nga considered that she would make ongoing changes with respect to rapport for individual weak students and adjust her delivery to suit them:

I will teach slowly and evaluate students' understanding of lessons and facilitate their engagement and self-learning from identifying strengths and weaknesses. (Nga-TRR2)

I will try to engage those left behind in learning. I have no perfect solution now. I think I got a simple solution that I will collect their email addresses to send supportive materials. (Nga-INT)

Nga increased confidence in teaching practice throughout the process because she felt more credible in teaching and was pleased with her performance:

In the last session of the course, my students organised a farewell party where they showed a video clip they made for me. Each of thirty-six students gave me a message that they like studying English more. One girl said: "I wish you will teach me in the future." I felt pleased. (Nga-INT)

Nga also indicated her willingness for collaborative learning: "I like to be observed and share teaching ideas with a peer more often" (Nga-INT).

In summary, Nga has been in the career for more than 13 years. Though she was concerned about students' and peer's feedback quality, she regarded the lenses as useful for her reflection. The intervention had positive impact on her reflection and changes in her belief about using evidence-based approach to teaching. Nga made some changes such as giving step-by-step, clearer, detailed instruction with a view to improve classroom excitement. She got a slight increase in end-of-term ratings on teaching organization, interest maintenance, and critical thinking encouragement. She became more confident in teaching and was willing to participate in POT with others. She considered POT a good chance to reflect from peer's critical feedback to identify weaknesses which she was unaware of and expected to have a more experienced peer. She preferred POT being organized for at least two semesters so that big changes could be facilitated. Nga's experience of the intervention is summarised in Figure 5.9. 


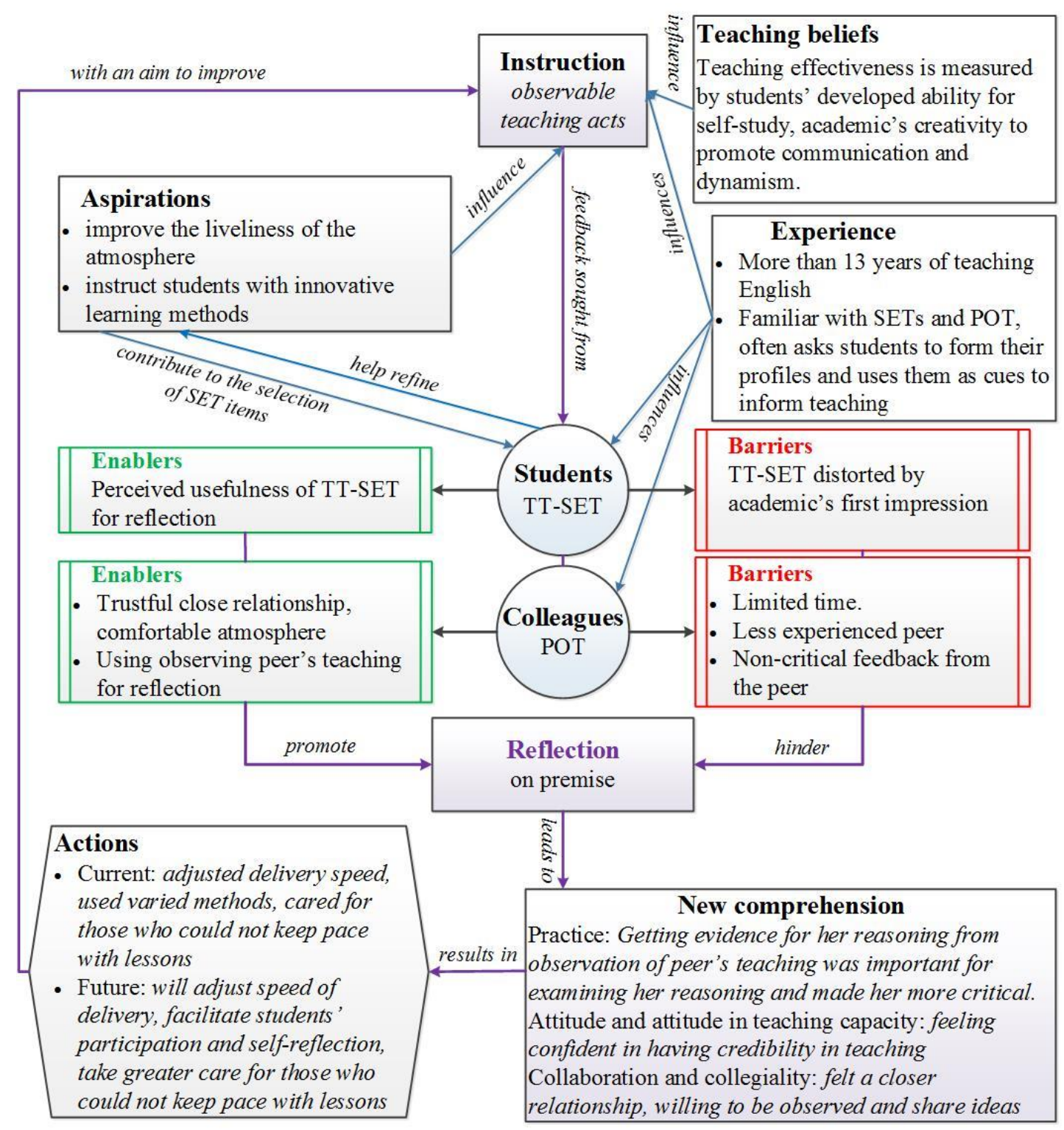

Figure 5.9: Summary logic model for Nga

\subsubsection{Summary}

Cuc, Giang, and Nga valued TT-SET, but Nga thought critique was necessary before use. Though they had a positive relationship with their peer, the three academics did not highly value their peer's feedback because they regarded them as junior peers. They believed that their peers just gave positive comment on their teaching. However, they admitted that observing their peer teach led to investigation of issues to consider for their own practice. They regarded themselves as experienced academics. It is likely that Cuc's and Giang's reflection focused on the process whereas Nga engaged in premise reflection. They all made changes despite reservations, gained confidence in their teaching capacity, and planned changes to future practice. Yet, it is possible that 
they were already competent and had a level of self-efficacy that helped them to be reflective. They seemed to get a benefit out of the intervention and use it as an opportunity to be self-reflective, which might be a quality they had before the intervention.

\subsubsection{Binh and Phung}

Binh has had about five years' teaching experience, and Phung has spent six years in teaching. The two academics were familiar with collecting SETs and often observed students' attitude and engagement to refine teaching. They had similar responses to the TT-SET and POT because they perceived the two lenses as useful for their reflection, but in some instances, they found their peer's feedback discouraging.

\subsubsection{Teaching beliefs and aspirations}

Binh believed that it was vital for academics to be pedagogically sound to facilitate classroom learning, including understanding students' needs, promoting communicative activities and being clear in delivery. According to Binh, an effective academic needs to be committed to teaching by improving pedagogical knowledge and being kind to students. She clarified: "A good and effective academic should work wholeheartedly on teaching, be good at educational methodologies, and always learn to improve teaching practices" (Binh-INT).

Binh also believed that students can engage in communicative activities where learning can occur through practice, developing comprehensive language skills:

My purpose is to develop students' communication competence. I help them not only grasp knowledge such as grammar but also use it. (Binh-INT)

After collecting the early-term SET, Binh developed strategies to use in her course to improve teaching as indicated in Table 5.10.

Table 5.10: Binh's aspirations and intended strategies

\begin{tabular}{|l|l|l|}
\hline Perceived teaching problems & \multicolumn{1}{|c|}{ Aspirations } & \multicolumn{1}{c|}{ Intended strategies } \\
\hline $\begin{array}{l}\text { needed to promote practice in } \\
\text { listening and speaking }\end{array}$ & $\begin{array}{l}\text { make speaking } \\
\text { activities } \\
\text { interesting }\end{array}$ & $\begin{array}{l}\text { - organising pair work and group work } \\
\text { and encouraging students to give } \\
\text { personal ideas and speak more English } \\
\text { - training their pronunciation }\end{array}$ \\
\hline $\begin{array}{l}\text { - needed to give clearer } \\
\text { explanations for vocabulary } \\
\text { and grammatical points } \\
\text { needed to provide students } \\
\text { with more exercises }\end{array}$ & $\begin{array}{l}\text { improve clarity of } \\
\text { communicating } \\
\text { ideas in lesson } \\
\text { presentations }\end{array}$ & $\begin{array}{l}\text { - helping students gain understanding of } \\
\text { new words } \\
\text { giving a variety of exercises to develop } \\
\text { their vocabulary }\end{array}$ \\
\hline
\end{tabular}


Phung's assumption about teaching is that, to boost learning interest, it is vital to facilitate real-life communication with fun in the classroom. As she said:

Most students like teaching organized via games. In listening, they liked to listen to news taken from BBC channel, movies, or songs. They like exciting atmosphere. They expect to learn more speaking so that they can communicate in future business environment. (Phung-INT)

Realising her students to be passive in learning, Phung thought that they needed stepby-step delivery of lesson content before being able to communicate in groups or pairs:

There is a part in this programme on "how to give a presentation." I wanted to give them clear instruction on how to structure a presentation before practicing in groups... I tried to finish "theory" before "practice." (Phung-DEB)

Phung set up a plan to improve teaching as listed on her pre-observation form as follows, combining student needs and her aspirations (Table 5.11).

Table 5.11: Phung's aspirations and intended strategies

\begin{tabular}{|c|c|c|}
\hline Perceived teaching problems & Aspirations & Intended strategies \\
\hline $\begin{array}{l}\text { needed to present lesson more } \\
\text { slowly and in more details }\end{array}$ & $\begin{array}{l}\text { - deliver lessons at an } \\
\text { appropriate level for } \\
\text { students } \\
\text { - increase constructive } \\
\text { feedback to students } \\
\text { - adjust speech flow when } \\
\text { delivering lessons } \\
\end{array}$ & $\begin{array}{l}\text { sharing teacher's realistic work } \\
\text { experience or creating rapport }\end{array}$ \\
\hline $\begin{array}{l}\text { - needed to give more time } \\
\text { speaking and listening } \\
\text { activities (i.e., encourage } \\
\text { communication in English) } \\
\text { - needed to provide students } \\
\text { with more homework and } \\
\text { reference materials }\end{array}$ & $\begin{array}{l}\text { - promote more practice on } \\
\text { pronunciation } \\
\text { - encourage students' } \\
\text { discussions in class and } \\
\text { sharing of ideas } \\
\text { - make presentations } \\
\text { interesting, using sense of } \\
\text { humour }\end{array}$ & $\begin{array}{l}\text { - organising group/pair-work } \\
\text { activities so that students can } \\
\text { get support from their peer } \\
\text { and the teacher } \\
\text { - giving rewards (bonus grades) } \\
\text { or punishment } \\
\text { - using sense of humour to } \\
\text { interest students and engage } \\
\text { them in learning }\end{array}$ \\
\hline
\end{tabular}

\subsubsection{Perceived values: both positive and negative}

Binh and Phung regarded TT-SET augmented with POT as useful for informing teaching, but they were concerned about the way peers gave feedback. They used SET to inform their teaching performance because they perceived it as reliable and worth considering: 
I think the early-term SET for my targeted area of teaching was good. They provided very specific ideas about my teaching. I think student feedback was appropriate and varied. (Phung-INT)

These academics used SET for reflecting on teaching and for guiding changes:

I respect students' ideas. As you know, there is an intangible teacher-student tie. If the tie is strong enough, academics will try to meet the needs. My students' attitude affected me a lot. I tried to see if they cooperated and made progress, or if my teaching contributed to their improvement. (Binh-DEB)

Binh found the feedback from one peer unhelpful because of peer judgmental feedback, and Phung found it discouraging because her peer gave feedback only on negative aspects of her practice without praise:

An wanted to give me feedback, but she said I did this and that is wrong. Meanwhile, my students told me that they could catch the points well and participated in speaking activities. It did not matter, you see, but she kept saying that way. (Binh-INT)

Binh had a second peer who gave constructive feedback and created a collegial relationship, and she indicated her satisfaction with the feedback and found it useful.

\subsubsection{Impact on pedagogical reasoning}

\section{Binh}

Binh's new comprehension focuses on effective use of time and facilitation of selfstudy. Through the first POT, Binh learnt new strategies to facilitate speaking activities, to time lessons appropriately, and to promote self-study:

I learned how to organise activities to promote speaking skill, make students more active, time lesson contents appropriately, save more time by quick grouping, and promote self-study. (Binh-TRR1)

With new strategies learned from the second peer, Binh changed the way she managed the class and adopted a style that engaged students in learning: "I stimulated students' interest and enthusiasm in learning" (Binh-TRR2). She also said:

After Cuc's advice, I could minimise dead time and save time for other activities.

I made a change on timing of classroom activities by grouping students more appropriately and setting time frames suitable for each activity. I was selective in getting feedback. (Binh-INT) 
The review of three videos provided evidence that Binh made some changes to her practice. During the first two videoed sessions, she had problems in class management with interactions, lack of detailed instruction for activities, and inappropriate sequencing of lessons with much dead time. In the third video, Binh stimulated interest in learning by organising varied activities, including games, exercises, and speaking activities with pair-work discussion and group-work presentation. She sequenced her lesson more appropriately; dead time was much reduced. Binh attempted to enhance clarity of teaching delivery; for example, she integrated with other activities teaching vocabulary, checking students' understanding of vocabulary, offering synonyms and Vietnamese translation during grammar exercises or the reading section. However, while she showed her enthusiasm in teaching during the first two videos, she appeared to be tired and did not move around to offer support during the third video (BinhVID3). The results of TT-SETs before and after the intervention remained unchanged (see Appendix N.9). From the researcher's viewpoint, Binh made changes to enhance practice, but her low level of enthusiasm, which might have resulted from her overloaded schedule (RJ (researcher journal)), may have affected students' interest in learning - the goal she wanted to achieve. The experience of the intervention led to Binh's intention to pursue a learner-centred approach and adjust instructional strategies. She said: "Next term, I will pursue the communicative approach and simplify lesson content so that students left behind can keep pace with others and join in communication" (Binh-INT).

Binh said she was confident in teaching. Dialogue about teaching with her peer affirmed Binh's teaching strategies as Cuc said: "Your teaching style is the same as mine" (Cuc-DEB). When both academics described similar problems and shared ideas about solutions, Binh was confident in her teaching skills: "When I organised teaching as what Cuc did, class time was used effectively... Learning from peer's feedback helped me deliver better presentations" (Binh-INT).

In summary, Binh has got more than 5 years' teaching experience in several institutions. She used students and peers as lenses for reflection: informing about teaching (i.e. identifying her strengths and weaknesses or important aspects of teaching that she was unaware of, and reconfirming teaching appropriateness). However, she believed that if colleagues' relationship was not good, feedback did not count. Learning came from an observer who was compatible in age and experience and knew 
how to give constructive feedback. The intervention had much positive impact on her reflection, helping her identify good or bad things of practice. However, heavy workload did not allow her to make timely changes to teaching even though she intensified more group-work activities in the late sessions of the course. She believed she made good changes to teaching. However, she paid deference to one of her peers in giving feedback and had a relationship breakdown with the other because of judgmental attitudes. This aspect is discussed in more depth in section 7.3.2.2. Binh's experience of the intervention is summarised in Figure 5.10.

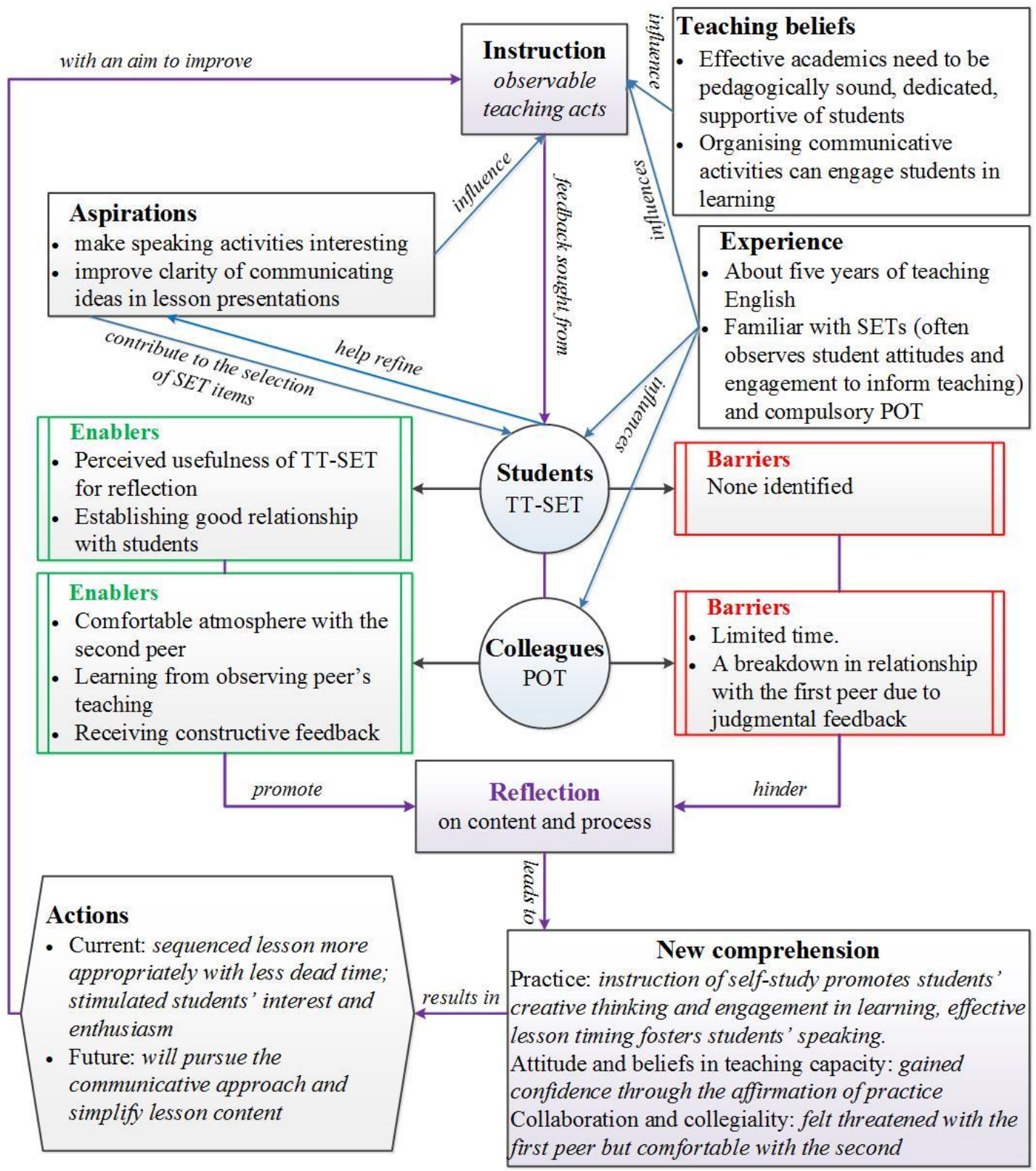

Figure 5.10: Summary logic model for Binh 


\section{Phung}

Phung's new understanding of practice emphasised the flexibility of teaching delivery. She learned that flexible teaching delivery, combined with entertaining activities, could make her students comfortable and interested in learning. She also perceived that encouragement of speaking interactions with "rewards and punishment" could promote student communicative activities:

Flexible presentations of lessons with entertaining activities could make students comfortable and interested in learning. I often encourage teacher-student and student-student communication using rewards and punishment. The communication will make students get used to speaking English. (Phung-TRR2)

Thanks to classroom observations and dialogue with her peer, Phung realised the value of asking students to prepare for lessons to have more time for speaking and using technology to vary methods that interest learners:

I learned Giang's way of organising classroom activities, grouping students, giving tasks, combining presenting lessons with games, and making classroom atmosphere exciting. (Phung-INT)

Phung's comprehension of new techniques and methods to enhance student learning resulted in changes of teaching practice. Phung explained:

I enhanced students' speaking activities in pairs or groups, designed tasks for group-work so that speaking skill could be promoted. I reduced "theoretical" content and organised games through using Power-point to create an interesting atmosphere and help students grasp lessons. (Phung-INT)

Considering areas requiring improvement suggested by the peer, Phung created more teacher-student communication by asking questions to check teaching appropriateness, facilitating students' sharing of ideas, giving clear instructions, and allowing more student-talking time. She intensified classroom communication and improved the learning atmosphere through watching her peer's presentation:

I have learned how to use sense of humour, present lessons beautifully and properly with PowerPoint, and organise games with the content. Since then, my teaching has been improved with application of games and more teacher-student and student-student interactions. The classroom atmosphere became more comfortable. (Phung-TRR2) 
These changes, which were not evident during the first two, were detected in the third video. Phung engaged students in the class activities. She used pictures and instructed the lesson more clearly, reduced dead time and teacher-talking time. She still used eliciting methods to encourage students to share their ideas and organised pair work and games to enhance their participation in discussion as in the first two videos. She appeared to be friendly and cheerful with smiles and jokes during the session. Her flow of delivery was appropriate and clear with step-by-step explanation, instruction, and feedback (Phung_-VID3). Students rated her significantly higher for end-of-term TTSET items related to interest stimulation, use of humour, and appropriateness of delivery (see Appendix N.10). The researcher believed that Phung achieved three of her goals and made effective changes to these aspects of practice.

Although stating the importance of using technology to enhance learning, Phung did not make changes regarding this for the course during the intervention; Phung promised next term changes to teaching by enhancing more communicative activities with fun through the use of technology. Her aim was to stimulate the students' interest:

Next term, I will use technology in teaching delivery with games, English movies, and songs which will lead to speaking activities. I will also set homework for them such as role-plays or dramas. I think these will make students more excited and students will use English for communication. (Phung_-INT)

Phung increased confidence throughout the process of TT-SET augmented with POT because of the belief that she had more credibility in teaching: "When I made changes with regard to student feedback, I realised that students were more interested and comfortable" (Phung-INT). She also had intention to share teaching ideas: "I like to have conversations about teaching with my peer" (Phung-INT).

In summary, Phung has been teaching for more than 5 years. She viewed TT-SET as a useful formative lens for reflection. She considered peer feedback via POT as an important opportunity to reflect and learn from others by identifying her strengths and weaknesses. However, she would like to have an observer who was enthusiastic and wholehearted in giving feedback. Phung made changes by using sense of humour, encouragement of spoken communication, reducing teaching 'theory' and organizing games with application of technology. She received higher ratings by students. Phung became confident in teaching and wanted to share teaching ideas with others and conduct POT. Phung's experience of the intervention is summarised in Figure 5.11. 


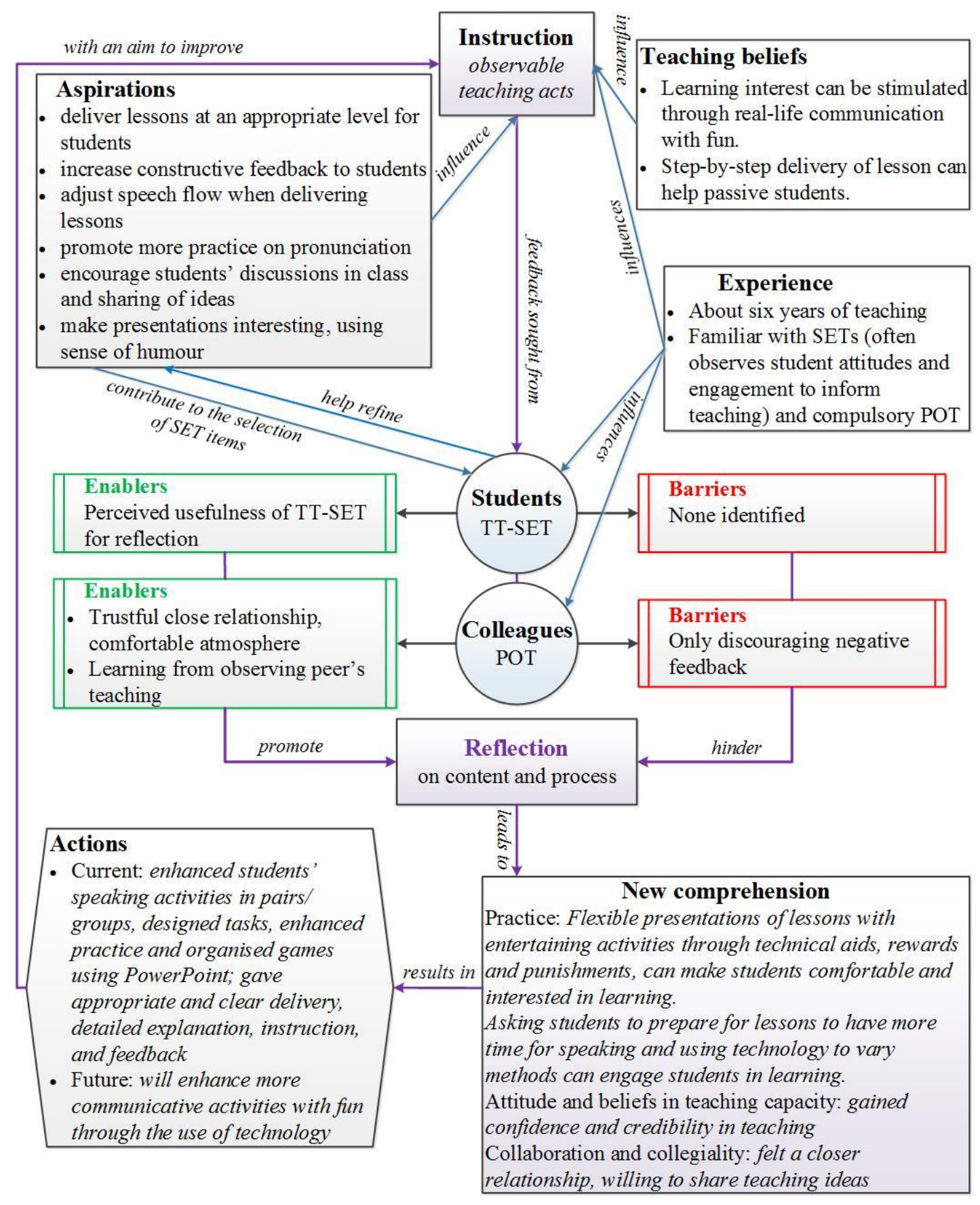

Figure 5.11: Summary logic model for Phung

\subsubsection{Summary}

Both academics regarded TT-SET as useful for their reflection whereas they were concerned about the way their peer gave feedback, which sounded judgmental or focused on negative comments. However, Binh had a positive experience with her second peer. Their negative experience with peer feedback may imply that lack of training in giving feedback may create negative feelings. However, both academics' reflection assisted them to make changes. Particularly, Phung made effective changes 
in some aspects that received higher end-of-term ratings. Both Binh and Phung felt confident in their practice and indicated they would continue to make changes.

\subsubsection{An}

An had more than 20 years' experience and felt confident in teaching. She often observed students' attitude to informing practice. TT-SET plus POT was not a positive experience for her because she did not value early-term TT-SET or the peer, whom she considered to be an inexperienced junior academic.

\subsubsection{Teaching beliefs and aspirations}

An emphasised the importance of content knowledge in effective teaching, which was viewed from the academic's role of 'a knowledge provider with a sense of humour':

Teaching delivery must ensure good content (e.g., grammar) and comprehensibility or clarity with a sense of humour. An academic can play the role of an actor/actress. If he/she cannot, he/she will fail to deliver lessons. Besides, knowledge of an academic should be beyond that of textbooks. Good students can understand lessons well, but weak ones cannot; thus, an academic with broad knowledge can help keep all students interested in learning. (An-DEB)

According to An, effective teaching also means how to keep learners engaged. Offering students extra materials was perceived as a means to motivate learners. She noted: "I often download materials for students" (An-INT). Based on her teaching beliefs without considering TT-SET, An set up a plan to improve teaching as summarised in Table 5.12.

Table 5.12: An's aspirations and intended strategies

\begin{tabular}{|l|l|l|}
\hline \multicolumn{1}{|c|}{ Perceived teaching problems } & \multicolumn{1}{c|}{ Aspirations } & \multicolumn{1}{c|}{ Intended strategies } \\
\hline $\begin{array}{l}\text { - needed to make lesson presentations } \\
\text { logical and systematic } \\
\text { - needed to deliver lessons at an } \\
\begin{array}{l}\text { appropriate level for students } \\
\text { - needed to enhance the clarity of } \\
\text { communicated ideas and information }\end{array}\end{array}$ & $\begin{array}{l}\text { improve ways of } \\
\text { organising } \\
\text { teaching }\end{array}$ & $\begin{array}{l}\text { - re-organising students for } \\
\text { participation in discussions } \\
\text { - checking students' attendance }\end{array}$ \\
\hline needed to give students feedback & $\begin{array}{l}\text { stimulate } \\
\text { students' interest } \\
\text { in learning }\end{array}$ & $\begin{array}{l}\text { - being more dynamic and } \\
\text { energetic in conducting lessons } \\
\text { - being more active to show } \\
\text { rapport and empathy } \\
\text { - being humorous }\end{array}$ \\
\hline $\begin{array}{l}\text { needed to consider the usefulness of } \\
\text { material content }\end{array}$ & $\begin{array}{l}\text { stimulate } \\
\text { students' creative } \\
\text { thinking about the } \\
\text { subject }\end{array}$ & $\begin{array}{l}\text { giving more updated detailed and } \\
\text { practical materials to motivate } \\
\text { students to learn and stimulate their } \\
\text { curiosity }\end{array}$ \\
\hline
\end{tabular}




\subsubsection{Perceived values: not beneficial}

An believed SET reliability increased with the time students studied with academics. She doubted the result of early-term SETs because she believed that a few teaching sessions did not allow students to give accurate feedback. As a result, she did not consider it in her plan to improve teaching. Instead, she found it useful to self-evaluate performance through observing student attitudes against her predetermined goals: "I do not care much about TT-SET, but I can know about student needs through observation which helps me know if students like my performance or not" (An-INT). She believed that the end-of-term TT-SET would be more reliable:

It is hard to say whether student feedback is $100 \%$ accurate. At the beginning of the course with just one or two sessions delivered, student ratings are inadequate. If the feedback is gathered after two-thirds of course time or at the end of the course, it could be more accurate, ranging from $70 \%$ to $80 \%$. (An-INT)

An also did not trust peer feedback and averred that observing her peer teach was not useful because she regarded her peer as a novice. An perceived that the reliability of peer feedback relied on a peer's degree of trustfulness and experience. She said: "My peer observer is too young and inexperienced to give feedback on my teaching" (AnINT). She believed that experience was the first criterion for a trusted and reliable peer because it was associated with the level of confidence in pedagogical capability. An thought that Binh was incapable of teaching effectively. She explained:

I have not learned anything from Binh for two reasons. First, she is inexperienced. I am much older than she is, and I have spent more years in the profession than she has. Second, I am capable of and natural in teaching even when being observed... Whether academics get the shivers down their spine under observation is important. If they do, everything will go wrong despite their good preparation. Binh was scared! (An-INT)

\subsubsection{Impact on pedagogical reasoning}

Because of An's perspectives regarding student and peer feedback, she did not take feedback into account to inform her practice. Instead, she observed students' attitudes, which helped her refine her teaching. An's new comprehension was that facilitating self-study would enhance learning interest, implying that traditional teacher-centred methods could bore students. She noted: 
In response to student needs through observation, I realised that facilitating selfstudy could motivate or interest the students in learning. For example, in the past, I used the "old" or traditional methods in teaching, students did not listen to my presentation, but now they excitedly look into studying matters more broadly even though they have known the issues. I can see the effectiveness. (An-INT)

An did not make any changes as planned. Her ways of organising teaching and stimulating learners' interest and creative thinking appeared to be the same. Her delivery was clear, but she did not actively engage students in learning as evidenced in the three videos. Students seemed bored with individual presentations because, while one student was speaking, others did not pay attention (An-VID3). The results of end-of-term TT-SETs indicated significantly lower ratings on seven teaching aspects (see Appendix N.11). From the researcher's perspective, An did not make changes to improve her practice. She did not take the peer's feedback seriously and had a breakdown in her relationship with Binh because she may have thought Binh's critique challenged her values (RJ). The interpretation of An's reluctance to use the peer's feedback was that she did not value Binh's feedback which lacked sensitivity related to power difference and may have turned into criticism. It seems more plausible than the interpretation that An thought she was good enough and did not need to improve. It was based on data noted in the researcher journal (e.g., An's motivation to participate in the intervention, changes of attitudes during the debriefing, and the meaning implied during the interview). Her awareness of practice was based on experience, which contributed to her rationalisation of her new comprehension. Further to this change in teaching beliefs, An intended to use technology to support teaching in the future despite not explaining this new understanding of practice. She said: "In the near future, I sure will use PowerPoint with pictures, which are specific/concrete and practical to interest students in learning" (An-INT). Her awareness of the usefulness of technology in teaching might have resulted from observing her peer's teaching because the peer often used PowerPoint in class (RJ). She said, "I have not used much 'slideshows' for my presentation" (An-INT).

An's confidence in teaching capabilities was not a result of the intervention process but from her experience. She said: 
In general, in terms of grammar, I do not need to prepare for it because I have been teaching for a long time and mastered it. As for generic knowledge, what the textbook covers is no problem to me. (An-INT)

In summary, An had more than 20 years' teaching experience. She considered earlyterm TT-SET unreliable and preferred to use observation on students' attitude to inform teaching. The intervention did not have much positive impact on her reflection, except that she changed her belief about using more technology in teaching. She received lower ratings for the end-of-term TT-SET. An felt self-confident in her teaching due to her experience. Although she did not have a positive experience with the intervention, she believed that students and peer lenses can be useful for teaching improvement. She experienced a breakdown in relationship with her junior peer and expected to have a more experienced professional or some observer who was compatible in age and experience. An's experience of the intervention is summarised in Figure 5.12. 


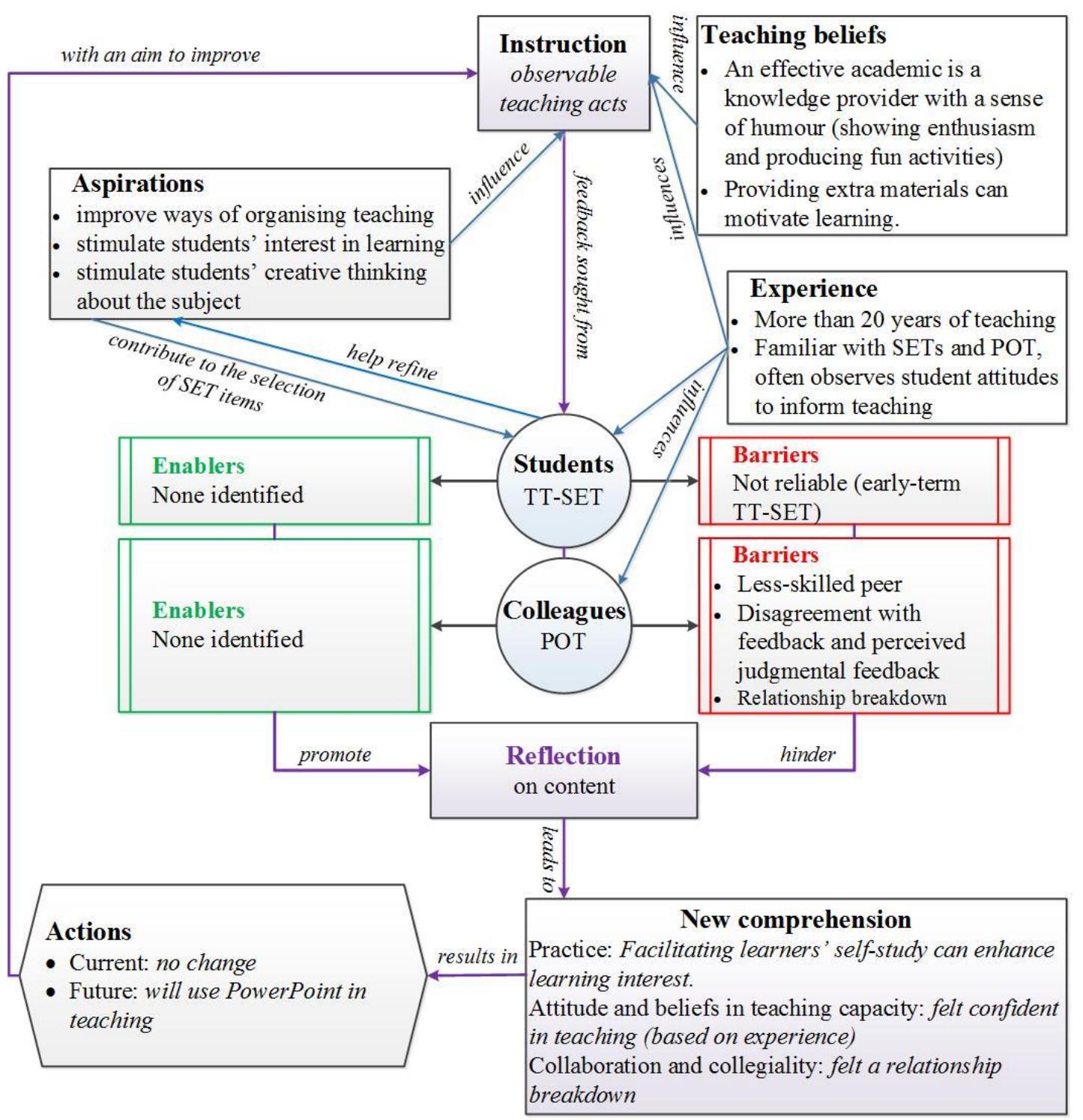

Figure 5.12: Summary logic model for An

An did not value student feedback because it was collected earlier in the course. This may imply that she confused the formative purpose with summative purpose of student feedback. She did not value her peer's feedback because she thought the peer was a novice and felt that the peer sounded judgmental. An's learning about teaching was based on her experience and her reflection was actually rationalisation of practice because she did not use external data for reflection, at least data from TT-SET and POT. She did not make changes to her teaching during the intervention. However, she intended to make changes to her future practice. 


\subsection{Summary of the chapter}

Each of the participants (the embedded cases within the overarching single case study) reflects diverse perceptions of the benefits of the intervention for their reflection on pedagogical reasoning. They varied in teaching beliefs and aspirations to improve teaching. Ten out of eleven participants appeared to be willing to engage in the implementation of TT-SET augmented with POT. They used student and peer lenses to guide changes in their teaching practice, which was illustrated within a summary logic model.

The TT-SET augmented with POT appeared to be a promising tool for professional learning and development. Academics were able to gain insights into their pedagogical practice and theory through student and peer feedback and teaching observation. As a result, they articulated their teaching beliefs and initiated actions to improve practice. This chapter, using cluster analysis where participants were grouped according to similar experience of TT-SET augmented with POT, provides a rich picture of the impact of the intervention process on individual academics. All the academics' stories will be drawn together to answer the research question in the following chapter. 


\section{Chapter 6. Addressing the Research Questions}

The individual stories described in Chapter 5 illustrate a complicated reality of the implementation of TT-SET augmented with POT in the research university. The findings in Chapter 5 were deduced by examining each participant independently. The second phase of data analysis involved examining data and findings across participants. The themes emerging from individual participants were examined across all the academics. Findings from individual academics' experiences are synthesised. An analysis of the synthesised data included examining each of the research questions across the participants. To give comprehensive answers to the main question: "In the context of a Vietnamese university, how does TT-SET augmented with POT impact on academics' pedagogical reasoning?" it is necessary to address the sub-questions through cross-participant thematic analysis. The questions are interrelated. To understand how the intervention impacts on academics' pedagogical reasoning (main question), it is necessary to document the perceived impact of the intervention on academics' new comprehensions with respect to areas such as practice, beliefs and attitudes (sub-question 1), reflection - the key mediator of these new understandings which may encourage their changes (sub-question 2), and challenges to its implementation (sub-question 3). Lastly, a summary is drawn from the analysis and synthesis.

\subsection{Perceived impact of the intervention}

Sub-question 1: What is the perceived impact of TT-SET augmented with POT on academics' practice, beliefs, and attitudes?

This section presents the changes in academics' process of pedagogical reasoning and action under the intervention. These include enhanced reflection and comprehension, action for current and future practice, and other outcomes.

\subsubsection{Reflection}

This section looks at academics' reflection and new comprehensions. The intervention prompted academics to reflect on or become aware of their practice. Through reflection, academics gained new knowledge on aspects such as practice and personal theories of English language teaching or reflective abilities. The ways in which the 
process helped them think about their teaching and make changes to their practice were the structured steps including identifying teaching problems through TT-SET, discussing them at the debriefing after reciprocal observation of peer's teaching, and writing reflection reports (see section 6.2).

Ten academics perceived the benefits of TT-SET augmented with POT for professional learning through the identification of techniques, strategies, and approaches that they thought would improve practice. Their gain in pedagogical knowledge centred on practice, context of practice, theory of practice, and reflective ability. Table 6.1 presents examples of the academics' reflection and new comprehension.

Academics' reflection is categorised, as shown in the second column of Table 6.1, based on Mezirow's (1991) content, process, and premise reflection. Content and process reflection is associated with problem-framing and problem-solving respectively, (i.e., focusing on problems in the situation and examining strategies and procedures to solve them). Premise reflection involves the questioning of one's beliefs and assumptions (i.e., addressing the why of teaching, the reasons for and the consequences of what one does (Mezirow, 2003)). In addition, it is suggested that reflection may lead to new comprehensions, and teachers' learning can be divided into: single-loop learning (the adoption of new action strategies to refine practice) and double-loop learning (the changing or complete shift of one's assumptions) (Argyris \& Schön, 1974).

All academics engaged in content and process reflection and single-loop learning, and three of them also engaged in premise reflection and double-loop learning (see Table 6.1). Content reflection can be illustrated in Hang's words: "POT made me reflect on teaching, i.e. critically identify what aspects of teaching are good and what needs improving" (Hang-INT). Hang identified some teaching problems as written in the peer observer's feedback form:

Detailed instruction should be given before carrying out activities such as discussions or exercises. It is necessary to encourage weak students to communicate by forcing them to talk instead of letting them volunteer for tasks. Giving some fun activities could inspire the students. The teacher should speak more slowly. (Hang-POF1) 
Table 6.1: Academics' enhanced reflection and other outcomes

\begin{tabular}{|c|c|c|c|c|c|}
\hline \multirow[b]{2}{*}{ Participants } & \multirow[b]{2}{*}{ Reflection } & \multirow[b]{2}{*}{ Comprehensions } & \multicolumn{2}{|c|}{ Action } & \multirow[b]{2}{*}{ Attitudes } \\
\hline & & & $\begin{array}{l}\text { Actual changes to current } \\
\text { practice }\end{array}$ & $\begin{array}{l}\text { Intended changes to future } \\
\text { practice }\end{array}$ & \\
\hline An & \multirow{7}{*}{ 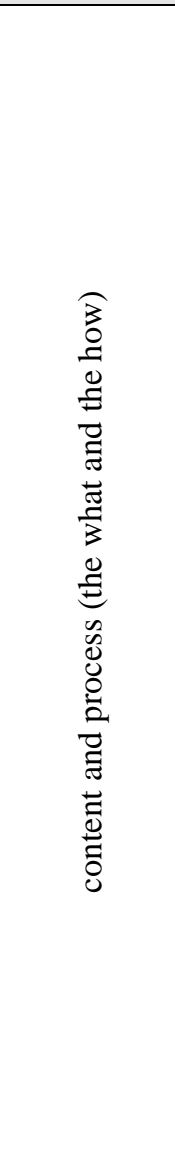 } & $\begin{array}{l}\text { Effective teaching requires facilitating learners' self- } \\
\text { study and use of technology. }\end{array}$ & no changes & will use technology in teaching & confident \\
\hline Binh & & $\begin{array}{l}\text { Instruction on self-study promotes students' creative } \\
\text { thinking and engagement in learning. Effective lesson } \\
\text { timing fosters students' speaking. }\end{array}$ & $\begin{array}{l}\text { stimulated students' interest and } \\
\text { enthusiasm } \\
\text { made a change in timing of } \\
\text { classroom activities }\end{array}$ & $\begin{array}{l}\text { will pursue the communicative } \\
\text { approach and simplify lesson } \\
\text { content }\end{array}$ & confident \\
\hline $\mathrm{Cuc}$ & & $\begin{array}{l}\text { Building good academic student relationships can } \\
\text { stimulate interest. Content knowledge is less important } \\
\text { than skill development. Appropriate level of content } \\
\text { supports the engagement of weaker students. }\end{array}$ & $\begin{array}{l}\text { integrated four language skills } \\
\text { in teaching delivery }\end{array}$ & $\begin{array}{l}\text { will promote comprehensive } \\
\text { language skills with basic } \\
\text { knowledge. }\end{array}$ & confident \\
\hline Dang & & $\begin{array}{l}\text { Interesting communicative activities promote learning. } \\
\text { Students need academics to listen to and address their } \\
\text { needs. }\end{array}$ & $\begin{array}{l}\text { used sense of humour, video } \\
\text { clips, and games to review } \\
\text { lesson }\end{array}$ & $\begin{array}{l}\text { will design a variety of } \\
\text { exercises, supply video clips } \\
\text { besides pictures, and connect } \\
\text { knowledge and reality }\end{array}$ & confident \\
\hline Phung & & $\begin{array}{l}\text { Rewards and punishments, flexible presentations of } \\
\text { lessons with entertaining activities through technical aids } \\
\text { can make students comfortable and interested in learning. }\end{array}$ & $\begin{array}{l}\text { enhanced students' speaking } \\
\text { activities in pairs or groups, } \\
\text { designed tasks for group work, } \\
\text { limited "theoretical" content and } \\
\text { organised games through using } \\
\text { PowerPoint to promote } \\
\text { interaction }\end{array}$ & $\begin{array}{l}\text { will use technology and } \\
\text { organise fun activities with } \\
\text { humour to encourage } \\
\text { communication }\end{array}$ & confident \\
\hline En & & $\begin{array}{l}\text { Effective teaching can be achieved through the use of } \\
\text { context in language teaching and sense of humour for } \\
\text { interactions. }\end{array}$ & $\begin{array}{l}\text { organised fun activities through } \\
\text { group-work } \\
\text { used context to introduce new } \\
\text { words }\end{array}$ & $\begin{array}{l}\text { will organise varied activities, } \\
\text { redesign textbook tasks, give } \\
\text { extra materials, give individual } \\
\text { support, facilitate cooperative } \\
\text { learning, and collect student } \\
\text { feedback }\end{array}$ & confident \\
\hline Giang & & $\begin{array}{l}\text { Content delivery needs more attention to avoid neglect of } \\
\text { important issues which are assumed to be easy for }\end{array}$ & $\begin{array}{l}\text { was careful in delivery of lesson } \\
\text { content }\end{array}$ & $\begin{array}{l}\text { will diversify activities to } \\
\text { motivate learners }\end{array}$ & confident \\
\hline
\end{tabular}




\begin{tabular}{|c|c|c|c|c|c|}
\hline & & $\begin{array}{l}\text { students, and individual support should be given to those } \\
\text { could not keep pace with the lesson. }\end{array}$ & $\begin{array}{l}\text { gave individual support to those } \\
\text { who had fallen behind }\end{array}$ & & \\
\hline Hang & & $\begin{array}{l}\text { Effective teaching requires being more flexibly selective } \\
\text { in content delivery (i.e., avoid complete dependence on } \\
\text { textbook content) and provide extra materials and clarity. }\end{array}$ & $\begin{array}{l}\text { made some adjustments to style } \\
\text { and subject matter: was more } \\
\text { enthusiastic, spoke more slowly, } \\
\text { gave clearer explanation of } \\
\text { concepts }\end{array}$ & $\begin{array}{l}\text { will promote students' } \\
\text { communication through } \\
\text { speaking and writing } \\
\text { will use learner-centred } \\
\text { approach to enhance students' } \\
\text { critical and creative thinking } \\
\end{array}$ & confident \\
\hline Khoa & \multirow{3}{*}{ 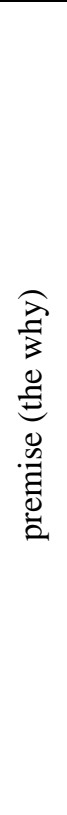 } & $\begin{array}{l}\text { Effectiveness requires innovative teaching (restructure } \\
\text { lessons with varied fun activities, promote self-study, and } \\
\text { care for individuals). Lessons and methods should be } \\
\text { more teacher-directed. Provide premises for future } \\
\text { application of knowledge and understanding of reality. }\end{array}$ & $\begin{array}{l}\text { timed lessons appropriately with } \\
\text { individual care } \\
\text { used funny stories to interest } \\
\text { students } \\
\text { focused on content knowledge } \\
\text { which was teacher-oriented }\end{array}$ & $\begin{array}{l}\text { will use competitive games or } \\
\text { music to engage students in } \\
\text { learning }\end{array}$ & confident \\
\hline Nga & & $\begin{array}{l}\text { A communicative approach should be combined with } \\
\text { traditional language teaching. Extra care should be paid } \\
\text { to weak students. } \\
\text { Getting evidence for reasoning through observations of } \\
\text { peer teaching was a way of becoming more critical in } \\
\text { reflecting on practice. }\end{array}$ & $\begin{array}{l}\text { adjusted delivery speed, used } \\
\text { varied methods, cared for those } \\
\text { who could not keep pace with } \\
\text { lessons }\end{array}$ & $\begin{array}{l}\text { will adjust speed of delivery, } \\
\text { facilitate students' participation } \\
\text { and self-reflection, take greater } \\
\text { care for those who had fallen } \\
\text { behind }\end{array}$ & confident \\
\hline Dieu & & $\begin{array}{l}\text { Reflection from students' and peer's feedback is } \\
\text { important for the changes to teaching. Making ongoing } \\
\text { changes to teaching make practice more effective and } \\
\text { students more interested. Task-based learning enhances } \\
\text { interest and cooperative learning. }\end{array}$ & $\begin{array}{l}\text { used meaningful tasks with } \\
\text { authentic language }\end{array}$ & $\begin{array}{l}\text { will promote task-based } \\
\text { learning with group or pair } \\
\text { interactions, on-the-spot } \\
\text { application of knowledge, } \\
\text { lesson-and-reality connection } \\
\text { will continue to make changes } \\
\text { and improve practice }\end{array}$ & confident \\
\hline
\end{tabular}

Note: Types of changes (Technical, Pedagogical, Critical) See section 6.1.2 
Then, Hang examined the strategies that would help to solve the problems. This is an example of process reflection, as Hang said: "I will present lessons more slowly, ask students questions to establish a habit of discussion, and help their critical and creative thinking" (Hang-TRR2). This is evidenced in the third video where Hang spoke more slowly in explaining new words and used an eliciting strategy to stimulate students' critical thinking to find word meanings (Hang-VID3). Therefore, Hang adopted new strategies to refine her teaching, and her teaching belief was modified as a result of reflection. She said: "In the past, I just stuck to the textbook content, but now I am more flexible and selective in teaching" (Hang-INT). Similarly, other participants engaged in content and process reflection and single-loop learning. For example, Giang thought about her practice by reconsidering the appropriateness of her teaching delivery for students. Dang considered the context of practice where students liked fun activities and she organised more interesting communicative activities. En gained an understanding that teaching vocabulary should be connected with their context of use. Binh learned that effective use of class time could allow more student communicative activities.

Three academics (Dieu, Nga, Khoa) engaged in premise reflection and double-loop learning. Dieu said: "I evaluated the effectiveness of my teaching activities in order to see if I should make complete changes or improve the existing activities" (DieuINT). She believed: "Solutions to the problems should be designed in different ways to see why this or that solution fits" (Dieu-INT). Dieu believed that trying different solutions to a problem more often helped to see which worked best. Dieu thought that making changes to teaching made her practice more effective and interested her students. It was also a way to know what students thought. This means she became aware of her teaching, and made changes to her teaching. This can be seen as adopting reflective practice in teaching.

I see trying making changes to teaching made practice more effective. My students became more interested because they had a chance to communicate ideas with one another through the changes. Then, I know them more. (Dieu-INT)

The reflection led Dieu to double-loop learning; she realised that reflection from students' and peer's feedback is important for the changes to teaching. She confirmed: "After getting feedback [from students and peer], I realised reflection is important for the changes" (Dieu-INT). Getting feedback from students could provide her with 
evidence in order to affirm whether or not changes are effective, and making ongoing changes and critically examining whether they were effective and why was perceived by Dieu as a way to improve practice. This can be seen as an evidence-based inquiry into practice, so Dieu's premise reflection was, after the intervention, that she became aware of the importance of reflection for changes to improve teaching.

Nga believed that she was using a communicative approach in teaching and assumed this approach would work well. However, after observing Hang's teaching and how she dealt with the students who could not keep pace with the lesson, Nga learned why these students could not engage in learning. Instead of using only a communicative approach, the grammar translation method can be used so that every student can learn. Nga believed that using a traditional method (grammar translation) in teaching could help her students learn in similar situations (as evidenced in section 5.2.3.3). By getting the evidence (from the peer's teaching) for her reasoning of practice and observations of peer teaching, Nga gained her new understanding that the peer lens helped her see how what she reasoned works in other classroom setting and she became more critical in reflecting on practice. Thus, Nga's premise reflection was that getting evidence for her reasoning from external sources such as observation of peer's teaching was important to the affirmation of her reasoning and made her more critical. She said:

By observing the peer's teaching, I know what combination of my approaches works. Then, I became more critical in analysing, for example, if these strategies work for students. I mean observations helped me find evidence for my reasoning for more appropriate applications of the strategies. So, my new knowledge about my teaching is that observing others makes me more critical in reflecting on teaching. (Nga-INT)

Similarly, POT stimulated Khoa's restructuring of belief in how to engage students in learning. Before, Khoa planned lessons to satisfy the students' preferred way of teaching: learning English with fun activities. However, she came to realise that organising fun activities did not always help them learn the necessary language skills: students could have fun, but they did not learn much. She said: "However, after POT, I realized that if the students were excessively served that way, I would fail to impart necessary knowledge" (Khoa-INT). Thus, she thought imposing her own ideas about teaching methods on students would help them learn: organising content and activities that she believed useful for their learning and future career. Khoa's belief changed 
from what she felt was 'student-directed' into 'teacher-directed' content, so her premise reflection was that delivering content she thought would be valuable for language skill development is more important than simply organising fun activities as students desired (see evidence in section 5.2.2.3).

An formed a special case. Her understanding of practice resulted from her experience. She believed facilitating students' self-study could enhance learning. Although An did not take notice of students' and peer's feedback or learn skills from her peer, the intervention somehow triggered her awareness of practice. She realised that using PowerPoint in lesson presentations could stimulate students' interest although she did not confirm this is what she learned from the peer.

Through the intervention, the academics' reflection on aspects of the what, the how, and/or the why of teaching seems have been stimulated or promoted, which led to their learning of new strategies to refine practice or changing of teaching beliefs or assumptions. The new comprehensions the academics identified may trigger actions to solve problems of current or future practice, which is presented in the following section.

\subsubsection{Actions}

The actions that academics actually took or intended to take may be categorised into technical, pedagogical, and critical changes as reported in Bell (2001). Only technical and pedagogical changes were observed in this study. Academics may have made more than one type of change, as summarised in Table 6.1.

Technical changes focused on technical adjustments (without changing teaching strategies or methods) such as timing classroom activities, using audio-visual aids, speaking slowly, and providing extra materials. For example, with new strategies from her peer, Binh reported her changes in her class management (e.g., grouping students). As noted in section 5.2.4.3, Binh made changes to her practice. Compared with her teaching in video 1 and 2, her teaching in video 3 had changed: Binh sequenced her lesson more appropriately, reduced dead time (grouped students quickly for discussion) (Binh-VID3). In the interview, Binh also confirmed that she reduced much dead time and grouped students for discussion quickly. Likewise, Khoa was responsive to her peer's feedback and started to solve the problem identified by Dang: "I think Khoa went through some sections so fast. It could be effective to use 
Vietnamese for explaining abstract vocabulary" (Khoa-POF1). Khoa made changes related to appropriate timing with care for those who could not keep pace with the lesson. The third video showed that Khoa reduced dead time and teacher-talking time by using varied activities to engage students in learning, and paid attention to individual students (Khoa-VID3). Hang also adjusted the speed of her lesson delivery to suit all students. The third video showed that Hang spoke more slowly to explain new words and ideas than before (Hang-VID3).

Pedagogical changes related to the pedagogical modification of teaching strategies or methods such as encouraging students' (English language) communication, collaboration skills (e.g., group-work, pair-work), developing students' critical and creative thinking, giving individual support, and strategies for motivating students in class (e.g., using games or music as a means to engage students in learning). Participants perceived that they had made pedagogical changes such as developing students' critical thinking, communication, and collaboration skills, strategies for motivating students in class, content sequence cohesion, strategies for facilitating students' reflection, and language skills. For instance, Binh reported that she tried to engage students in learning, and the third video-recording showed that she used varied activities (e.g., games) to stimulate students' interest in learning (Binh-VID3). Cuc gained insights into practice by exploring the effectiveness of her pedagogy. She changed her practice to suit student needs:

I changed or organised more classroom activities. I followed my students' preferred way of teaching. When they wanted to be trained with listening, I gave more listening tasks... At first at the beginning of the course, I did not pay attention to training integrated skills, but now I do. (Cuc-INT)

Cuc emphasised the necessity of integrating four language skills (listening, speaking, reading, and writing) rather than transferring content knowledge. The third video showed that Cuc scheduled teaching these language skills in her session. For example, the third video showed these steps: students were asked to share ideas about advantages and disadvantages of arbitration and litigation in groups; one student was asked to read a text from the book out loud and find the meaning of key words; students were also asked to rewrite the reading text using plain English; Cuc then explained listening strategies for the listening task, which seemed to be effective because many students could do the task (Cuc-VID3). Phung adjusted her practice after recognising 
student needs, watching her peer teach and receiving peer feedback. The changes that Phung made were based on her understanding of students' preferred way of teaching: facilitating real-life communication with fun in the classroom. Phung's actions included as having more teacher-student communication by asking questions to check teaching appropriateness, facilitating students to share ideas, giving clear instructions, and allowing more student-talking time resulted from her peer's feedback (PhungVID3). In the interview, Phung also mentioned that she intensified classroom communication and improved the learning atmosphere. Receiving Phung's feedback and observing her teach led to Giang's thinking of practice that facilitated timely adjustment to teaching. In the interview, Giang said that she refined her practice by paying attention to her style and delivery of lesson content. Dang's new comprehension that fun activities could stimulate learning interest led to her adjustment of practice. Learning from the peer's limitations in teaching, Nga identified the peer's aspects of teaching that needed improving and figured out solutions for her own problems. Caring about those students who could not keep pace with others when using the communicative approach, Nga also used a traditional method (e.g., translating a reading message (Nga-VID3) so that weaker students could understand it). Hang responded to suggestions by her peer, Nga, to solve her identified problems of teaching: clarity of instruction, management of students' communication, and organisation of interesting activities. Dieu engaged students in meaningful tasks with authentic language. For example, she set up a competitive game by showing a picture depicting an event in native speakers' setting and asking groups of students to build as many passive voice sentences as possible (Dieu - VID3). She believed that facilitating more interactions among students through games, guiding questions, and the like would stimulate students' interest in learning. These examples indicated that most academics made pedagogical changes.

Critical changes related to trying completely different practice such as the adoption of reflective practice as an ongoing strategy for teaching improvement (e.g., collecting SETs or learning from colleagues) or adopting quite different teaching methods. Critical changes were not observed in this study. However, there were two instances whereby academics intended to make critical changes to future practice. For example, with a changed view that ongoing learning enhanced teaching effectiveness, Dieu intended to join in collaborative learning with colleagues. Similarly, collecting TT- 
SETs is En's strategy to find evidence for her practice. These intentions imply that they regarded reflection as a mechanism for making changes to improve teaching and may adopt reflective practice in the future. One of the possible explanations for the lack of observed critical changes could be the short time span (which will be discussed later). For example, Nga gave her opinion: "A few weeks between POTs was not enough for major changes" (Nga-INT).

In addition to changes to current practice, the academics also wanted to make changes to their future practice. Their intention was also varied with technical, pedagogical, and critical planned changes. For example, En intended to provide students with more materials. Dieu said she would make pedagogical changes to her teaching in the direction of communicative language teaching with the focus on task-based language learning. Giang intended to refine her teaching strategy to encourage students' interest in learning in the future: "I will diversify classroom activities to motivate learners" (Giang-INT). The intention to make critical changes was reported earlier. These intentions indicate that the academics were thinking about their actions to solve problems in future practice. An did not make actual changes to current practice, but intended to use PowerPoint in future practice.

In comparing these actions with the academics' new comprehensions resulting from reflection (see Table 6.1), it is possible to say that new understanding can trigger academics' action for solving problems of current and future practice with an aim to improve teaching.

\subsubsection{Attitudes: increased confidence and self-efficacy}

Self-efficacy refers to one's belief in his/her capabilities to execute behaviours necessary to produce specific performance attainments (Bandura, 1977a, 1986, 1997). Ten participants reported increased confidence and many of them may have developed a sense of self-efficacy through their new understandings of their teaching and actions to refine practice.

Cuc, Dieu, En, Phung, Giang, and Nga reported that they developed confidence in their teaching. Cuc reported that feedback from her students and peer helped affirm her teaching methods. Cuc gave an explanation as to why she believed she could manipulate her influence on students' learning: 
Expectation? I think my students love me because they realised how enthusiastic and passionate I am [in lesson preparation and teaching] for their better learning outcomes. I believe that students may feel ashamed if they do not try their best. (Cuc-INT)

Cuc said early in the course she thought about trying to integrate four language skills in teaching (i.e., in teaching a skill such as writing, Cuc designed a session that developed speaking, listening, and reading skills with a focus on writing) and applied her strategies a few weeks later. Then, she saw that she improved student attendance. She said she did not check attendance, but students came and rarely played truant (in the university context, checking attendance is perceived as a way to force students to attend the classes, and students' course scores can be deducted for truancy). This claim is an example that implies Cuc's mastery experience of her practice. Dieu and En had a feeling of "being better" and wanted to join in future collaborative learning to teach. Dieu expressed her confidence: "I see trying making changes to teaching made practice more effective. My students became more interested because they had a chance to interact with one another. Through the changes, I know them more" (Dieu-INT). Besides feeling confident, Dieu developed a belief that she can make a change in students' learning: "I believe I can manipulate impact on students with well-prepared lessons" (Dieu-INT). This signals the development of self-efficacy. En believed she could have made a difference in student learning if she had not been involved in an accident: "I think I have tried to make good lesson plans and teach more actively in order to promote interest and engage students in learning. The outcomes could have been better if I were not injured [in the middle of the course]..." (En-INT). Phung, Giang, and Nga felt that they had more credibility in teaching. Phung believed that students were more interested in her lesson when she made changes. Giang had more certainty of her belief about teaching from applying her strategies successfully, and she said: "I realise that I taught more successfully this semester than before because I invested much time in lesson plans with varied activities, e.g., some useful games to help students learn" (Giang-INT). Nga believed that because of the changes she made, student end-of-term ratings "will sure be higher" (Nga-INT). Nga's words show not only her confidence in performing her teaching well but also her belief in her capability of making a difference. 
Three others - Dang, Khoa, and Hang - developed a belief in their ability to use a new strategy from vicarious experience or modelling which then motivated them to try the new teaching idea or become more creative. Dang felt confident in her teaching because she learned good techniques from her peer. She said: “...my teaching this term could not have been good without POT... I learned a good way to review lessons" (Dang-INT). Hang had similar vicarious experience: “[I] learned good things from observing her [Nga's] teaching" (Hang-INT). Likewise, Khoa was confident in her pedagogy. Also, Khoa believed that she understood the teaching context well and could exert control over it and make students learn. She said: "I believed understanding the context can help me make it [orient what students should learn rather than organise fun activities as they wish]" (Khoa-INT). Khoa's claim signals her enhanced selfefficacy.

Peers' verbal feedback may contribute to some academics' learning and changes, and they gained confidence when they implemented successfully what was suggested. Binh changed her practice after Cuc's advice and thought it was more effective: "Cuc suggested I try this way... After Cuc's advice, I could minimise dead time and save time for other activities..." (Binh-INT). An reported that she felt confident in her teaching based on her experience, not from learning through the intervention process.

These participating academics reported a greater awareness of themselves and their pedagogical reasoning that brought about positive attitudes they had about their capabilities. Most of them felt confident, and the evidence shows that the intervention brought about mastery experiences, vicarious experiences, and verbal persuasion (Bandura, 1977a, 1997). These are possible sources of teachers' sense of efficacy. Though not all participants indicated a clear-cut enhanced self-efficacy, several of them, namely Cuc, Dieu, En, Nga, and Khoa signalled the enhancement of selfefficacy, their beliefs in their capabilities to take action and manipulate students' learning.

\subsubsection{Enhanced collegiality and collaboration}

The development of collegiality and collaboration between peers appeared to contribute to the usefulness of reciprocal observation and debriefing. Though challenges existed in the peer relationship perceived by three academics (see section 6.3.2.2) who did not develop collegiality and collaboration, sharing ideas about 
teaching and observing peers teach was perceived by eight participants to nurture collegiality between peers. These eight participants reported that POT made their peer relationships develop to a new level.

For example in the pair Dang-Khoa, Dang perceived a trustful and cooperative atmosphere with Khoa: "I trust Khoa. She is so nice" (Dang-INT). She also said she wanted to discuss teaching ideas and carry out reciprocal observation with Khoa: "I like to go on sharing teaching ideas and doing observations with Khoa" (Dang-INT). The good relationship between the peers influenced how they perceived their peer's feedback, which facilitated reflection on practice and the course programme:

Dang is out-going. I felt pleased to do POT with her... Dang's feedback was more helpful: more constructive and more formative [i.e., more useful than TT-SET]. Because my peer knows pedagogical content knowledge and the programme, she could give more precise feedback on teaching effectiveness. (Khoa-INT)

The peers also collaborated after POT. Collegiality was sometimes shown by the peers sharing sources of teaching materials. For example, Dang said: "Khoa gave me some video-clips for teaching and showed me some useful websites where I can find useful teaching materials." (Dang-INT).

A similar atmosphere developed between Dieu and En, due to their willingness to give feedback and learn from each other. POT drew these peers closer, leading to a closer friendship. Dieu said: "POT makes learning to teach more effective and peers closer. When giving feedback, we got in harmony with each other" (Dieu-INT).

As presented in section 5.2.2.3, Dieu said that she would continue improving teaching so that other colleagues can learn from her and she can learn from them. This means she was willing to act as a support person and model teaching. Similarly, Khoa wanted to share teaching ideas and be observed by peers. She said: "I want to share my teaching ideas with others and would like them to observe my teaching because this collaboration with colleagues helps me reflect on teaching and gain new understanding" (Khoa-INT). Academics believed that classroom visits and peer feedback in mutual talks were valuable to self-directed learning. As these peers developed a strong collegial relationship that facilitated constructive feedback, they believed that they have improved important aspects of teaching. For example, En said: 'I learned Dieu's ways to stimulate students' critical thinking and organise effective and fun activities to engage students in learning" (En-POF1). 
The pair Giang-Phung felt a closer friendship than before. Phung reported that they were open in conversation about teaching and truthful about each other's teaching problems. She said: "Our relationship is quite good. In general, we were open and truthful in giving feedback without apprehension" (Phung-INT). She also emphasised: "Because we are close friends, we were open and truthful with each other" (Phung-INT). Giang perceived the closeness with Phung: "After spending much time in conversation about teaching, I felt Phung was closer to me" (Giang-INT). The evidence shows that the peer relationship between Giang and Phung became better although there was an instance that Phung commented that "Giang's feedback just focused on what I was not good at" (Phung-INT).

The pair Nga-Hang gained better understanding of each other. Although Nga thought Hang might have paid deference to her in giving feedback, she perceived a better mutual understanding with Hang: "I thought Hang paid deference to me [at the debriefing]... Now I think our relationship is just improved" (Nga-INT). Hang conveyed her feeling about the peer relationship: "I better understand Nga. We often shared ideas about family, life, and teaching. It is even better when I observed her teaching" (Hang-INT).

The development of a collegial relationship can be viewed as the willingness to share teaching ideas and beyond, modelling teaching, sharing of teaching materials, and mutual understanding. Collaborating in the intervention process in the examples above encouraged the development of better peer relationships which enhance academics' willingness to learn from colleagues in order to improve their teaching.

\subsection{Academics' perceptions of the intervention}

Sub-question 2: What are academics' perceptions of the effectiveness of TT-SET augmented with POT for promoting their reflection in the process of pedagogical reasoning?

This section presents academics' perception of TT-SET and POT on promoting their reflection. Although there were different views of the values of the intervention, many participants indicated that all the structured steps were useful for their reflection. They also indicated particular steps they liked the most. 


\subsubsection{TT-SET as a possible promoter of reflection}

Academics were divided in their perception of TT-SET with respect to how it helped them learn about teaching. While five academics expressed their concern about TTSET, six others perceived that it was valuable for reflection and guiding changes (see Table 6.2).

Table 6.2: Perceived values of TT-SETs

\begin{tabular}{|c|c|c|c|}
\hline Participants & Valuable? & Reliable? & What for? \\
\hline $\begin{array}{l}\text { Binh, Cuc, } \\
\text { En, Phung, } \\
\text { Giang, Dang }\end{array}$ & valuable & $\begin{array}{l}\text { Reliable: trustworthy, } \\
\text { diverse, formative }\end{array}$ & $\begin{array}{l}\text { - informing teaching and reflecting } \\
\text { (e.g., knowing what students need } \\
\text { and think, their reaction, and } \\
\text { learning from experience) } \\
\text { - } \text { facilitating changes }\end{array}$ \\
\hline $\begin{array}{l}\text { Dieu, Hang, } \\
\text { Nga, Khoa }\end{array}$ & $\begin{array}{l}\text { having } \\
\text { limited } \\
\text { value }\end{array}$ & $\begin{array}{l}\text { Limited reliability: } \\
\text { requiring reconsideration } \\
\text { before use because of } \\
-\quad \text { student carelessness } \\
-\quad \text { subjective thinking } \\
-\quad \text { first impression } \\
-\quad \text { lack of responsibility }\end{array}$ & $\begin{array}{l}\text { - informing teaching and reflecting } \\
\text { - facilitating changes }\end{array}$ \\
\hline An & $\begin{array}{l}\text { not } \\
\text { valuable }\end{array}$ & $\begin{array}{l}\text { Unreliable: doubtful of the } \\
\text { accuracy of early-term } \\
\text { SET }\end{array}$ & no use \\
\hline
\end{tabular}

One academic rejected the early-term TT-SET because of her doubt about its reliability and her confidence in her practice. She regarded observation of students' attitudes as an accurate means of knowing whether students were satisfied with her teaching performance. The academic believed that she could teach well without attention to student feedback so long as she showed adequate enthusiasm. Four others who regarded it as an additional source of information on teaching carefully considered its appropriateness before use. They thought that SET reliability was low due to student carelessness, subjectivity, first impression, and lack of responsibility and wholeheartedness. However, they still considered collecting TT-SET as useful because it helps them know what their students need and adjust teaching. For example, Dieu said: "I found TT-SET practical because it can help me understand the minds of students, what they expect from my teaching, and then it can help me adjust my practice" (Dieu-INT).

Six academics regarded TT-SET (as well as SET in general) as a reliable way of informing teaching to meet students' expectations. They described TT-SET as informative, supportive to teaching, useful, and important. One of them emphasised 
the essential role of TT-SET by regarding students as 'customers'. TT-SET was perceived as one of the lenses for academics to self-evaluate their practice and take initiatives for change. These academics used TT-SET for reflecting on their present performance against past performance (i.e., practice before TT-SET collection). For instance, Dang said: "Student feedback is worth considering for self-evaluation" (Dang-DD). Dieu explained how TT-SET could help her: "In order to teach well, the more opinions I can collect from students, the more effective is my teaching" (EnINT). Knowing students' experiences of teaching helped academics identify what changes in practice were needed. Dang explained: "TT-SET guided me to make changes to teaching practice appropriate for students" (Dang-INT). Nga also mentioned TT-SET as a guide for changes to practice: "TT-SET can help me see students' reaction to teaching to thereby adjust my speed of speech or the way I communicate ideas" (Nga-INT).

Participants also reported that they used other means such as observing students' attitudes, free-written feedback, written-down early-term expectations, an orientation session, and interviews to justify their understanding of student needs before refining their practices. These academics considered that responding to student feedback was a way to improve teaching. Ten participants perceived that their students preferred a lively way of teaching (e.g., interesting, practical, communicative, interactive, comfortable, experiential, and entertaining), which they mostly learned from TT-SET. For instance, Dang realised that her students liked to do communicative activities for bonus grades. She said:

From TT-SET, I was surprised that students liked group-work activities for bonus grades so much. They tried to engage in the activities by giving as many ideas as possible to receive bonus grades. (Dang-INT)

The evidence shows that though there were concerns about the reliability of TT-SET among four academics (who expressed concerns but still considered it worth using) others considered it useful as a possible reflective lens for examining and then making changes to refine teaching.

TT-SET promoted their autonomy and self-directed learning about teaching as valued by nine academics. According to Knowles (1975, p. 18), self-directed learning refers to a process: 
... in which individuals take the initiative, with or without the help of others, in diagnosing their learning needs, formulating learning goals, identifying human and material resources for learning, choosing and implementing appropriate learning strategies, and evaluating learning outcomes.

They believed that TT-SET was valuable for its formative information on targeted areas of teaching practice (see Table 6.3):

I like TT-SET for its focus on what I consider. It shows my strengths and weaknesses for making changes. S-SET provides general information that I do not know what changes should be made, informing me just low or high ratings. (Dang-INT)

One academic emphasised that TT-SET was appropriate because S-SET used for all majors could not reflect the needs of students in a particular major:

In my opinion, TT-SET is more appropriate. Students in economics have different needs compared to students from other majors. (En-INT)

Giang and Nga valued TT-SET but also mentioned it might fail to provide comprehensive information or aspects of which they were unaware. Giang needed both general information and specific focused information, whereas Nga thought TT-SET might not reflect important aspects requiring improvement:

I like TT-SET because of its focus on needed information while S-SET applies for all disciplines. However, using TT-SET may lead to a lack of important aspects that need considering because academics have a tendency to choose items based on their strengths and students' needs. (Nga-INT)

Binh and Hang preferred S-SET to TT-SET because the former provides more valuable comprehensive information while the latter contains teacher-selected items likely to be on areas they were good at and may lack some important aspects of teaching that they were unable to identify.

I think TT-SET is more suitable to me. Well...but now I prefer the standard one because it is comprehensive. With the former, academics often have a tendency to choose good aspects of practice for POT. They will conceal the bad side of things. Nobody is courageous enough to let others observe what they are not good at... There is something we may not pay attention to. With the latter, when people observe our teaching, they may discover it and give surprising feedback. (Binh-INT) 
Hang also added that she needed a disciplinary S-SET with more valuable information on a subject area rather than a general S-SET for all disciplines: "I like S-SET for English because TT-SET may not reflect other important aspects that I am unaware of" (Hang- INT).

Nine valued TT-SET and seven participants preferred it over S-SET because it helped them diagnose their teaching problems, or learning needs (i.e., diagnosing the teaching areas they want to improve). Then, based on the information from TT-SET, they can choose appropriate strategies to improve these areas. For example, Cuc said: "This will help me plan to teach appropriately" (Cuc- INT). Khoa also confirmed that TT-SET help her solve teaching problems together with peer's support:

I think the TT-SET is more valuable to me. It can help me to solve prioritized weaknesses to meet students' needs, which can be achieved with help from a peer, especially an experienced one. In short, the TT-SET facilitates step-by-step solution of teaching problems. (Khoa- INT)

Table 6.3: The role of the TT-SET in academics' self-directed learning

\begin{tabular}{|c|c|c|}
\hline & \multicolumn{2}{|c|}{ Comparison } \\
\hline & TT-SET & S-SET \\
\hline \multicolumn{3}{|c|}{ Preference of TT-SET } \\
\hline An & $\begin{array}{l}\text { provides formative information on teacher- } \\
\text { considered aspects of teaching }\end{array}$ & provides unwanted information \\
\hline $\mathrm{Cuc}$ & $\begin{array}{l}\text { provides targeted detailed information on } \\
\text { teaching practice to plan teaching } \\
\text { appropriately }\end{array}$ & is not useful for everyone \\
\hline Dieu & informs teacher-targeted aspects of teaching & does not provide focused information \\
\hline En & $\begin{array}{l}\text { allows academics to get targeted appropriate } \\
\text { information on student needs in a particular } \\
\text { discipline }\end{array}$ & $\begin{array}{l}\text { does not provide teacher-directed } \\
\text { information and meet subject standard for } \\
\text { evaluation }\end{array}$ \\
\hline Phung & $\begin{array}{l}\text { provides accurate information on areas } \\
\text { academics need to focus on }\end{array}$ & $\begin{array}{l}\text { does not provide teacher-needed } \\
\text { information }\end{array}$ \\
\hline Dang & $\begin{array}{l}\text { offers teacher-targeted information on } \\
\text { teaching }\end{array}$ & $\begin{array}{l}\text { gives general information and academics } \\
\text { do not know what changes should be } \\
\text { made, informing just low or high ratings }\end{array}$ \\
\hline Khoa & $\begin{array}{l}\text { helps to solve prioritised weaknesses } \\
\text { i.e., offers focused information on teaching, } \\
\text { facilitates step-by-step solution for teaching } \\
\text { problems }\end{array}$ & $\begin{array}{l}\text { does not provide teacher-targeted } \\
\text { information }\end{array}$ \\
\hline Giang & offers specific focused information & offers general information about practice \\
\hline Nga & $\begin{array}{l}\text { offers targeted information on teaching, but } \\
\text { could lack important issues }\end{array}$ & is applied for all disciplines \\
\hline \multicolumn{3}{|c|}{ Preference of $S-S E T$} \\
\hline Binh & $\begin{array}{l}\text { contains teacher-wanted items on areas they } \\
\text { were good at, } \\
\text { lacks some unaware areas with TT-SET }\end{array}$ & more valuable comprehensive information \\
\hline Hang & does not provide comprehensive feedback & $\begin{array}{l}\text { reflects other important aspects that } \\
\text { academics are unaware of }\end{array}$ \\
\hline
\end{tabular}


In summary, TT-SET was mostly regarded as a useful tool that facilitated academics' self-directed learning because it allowed them to target teaching aspects for feedback and to act on them for improvement. However, there was also a need for comprehensive information on teaching that only S-SET could provide. Academics preferred TT-SET because it met their needs for autonomy in refining teaching practice which was associated with self-regulated learning about teaching. In other words, academics' preference for TT-SET was influenced by the degree to which they wanted to be autonomous.

It should be noted that it was not possible to document academics' reactions to the end-of-term TT-SET results because the tight schedule made the interviews come before the results were produced and sent to participants. However, I documented their expectations of the end-of-term TT-SET results as described in Table 6.4.

Table 6.4: Academics' expectation of end-of-term TT-SET results

\begin{tabular}{|c|c|c|c|c|}
\hline \multirow[t]{2}{*}{ Participants } & \multicolumn{2}{|r|}{ Expectations } & \multirow{2}{*}{$\begin{array}{l}\text { Actual } \\
\text { TT-SET } \\
\text { results }\end{array}$} & \multirow{2}{*}{$\begin{array}{c}\text { Comments on if } \\
\text { expectation } \\
\text { matches TT- } \\
\text { SET results }\end{array}$} \\
\hline & $\begin{array}{l}\text { Expected } \\
\text { ratings }\end{array}$ & Reasons & & \\
\hline An & higher & teacher great enthusiasm & lower & different \\
\hline Binh & unchanged & $\begin{array}{l}\text { did not improve much due to } \\
\text { heavy workload }\end{array}$ & unchanged & similar \\
\hline Cuc & unchanged & maybe lack of student attention & unchanged & similar \\
\hline Dieu & don't know & $\begin{array}{l}\text { but students seemed more } \\
\text { interested }\end{array}$ & unchanged & not applicable \\
\hline En & unchanged & $\begin{array}{l}\text { higher unless the teacher showed } \\
\text { enthusiasm and movement (were } \\
\text { injured and unable to stay active) }\end{array}$ & unchanged & similar \\
\hline Phung & higher & $\begin{array}{l}\text { having improved practice and } \\
\text { achieved the goals }\end{array}$ & higher & similar \\
\hline Giang & unchanged & no reasons (just thinking so) & higher & different \\
\hline Hang & $\begin{array}{l}\text { unchanged } \\
\text { or lower }\end{array}$ & $\begin{array}{l}\text { feeling students' attitudes were } \\
\text { the same }\end{array}$ & higher & different \\
\hline $\mathrm{Nga}$ & higher & $\begin{array}{l}\text { realised students were more } \\
\text { interested and engaged }\end{array}$ & unchanged & different \\
\hline Dang & lower & $\begin{array}{l}\text { lacked experience for teaching } \\
\text { the course }\end{array}$ & unchanged & different \\
\hline Khoa & don't know & $\begin{array}{l}\text { difference between teacher } \\
\text { teaching intention and students' } \\
\text { expectation }\end{array}$ & unchanged & not applicable \\
\hline
\end{tabular}

Three academics (Phung, Nga, and An) believed they would receive higher student ratings because they thought that they became more effective towards the end of the course. Phung said: "I think students will rate my teaching higher because I believed that I have achieved the teaching goals and improved practice" (Phung-INT). Nga 
believed: "I think student ratings will be higher because I realised they were more interested and engaged in learning" (Nga-INT). An reasoned: "The end-of-term TTSET is long enough for accurate evaluation, and with my enthusiasm in teaching I think students may rate my teaching higher" (An-INT). However, only Phung's expectation matched the TT-SET results. Nga received unchanged ratings compared to the early-term TT-SET while An received much lower ratings. Five academics (Binh, Cuc, En, Giang, and Hang) expected unchanged end-of-term TT-SET results. They gave a number of reasons for this such as their insufficient efforts and students' not paying enough attention. For example, Binh said: "During that period [the intervention] I felt tired, was busy with a tight schedule, and did not try my best effort in teaching, so I do not expect higher ratings" (Binh-INT). However, three expectations matched with the TT-SET results, while Giang and Hang received higher ratings. Two academics were not sure how students would react to their changes. Dieu guessed: "I do not know what the result will be, but my students seemed more interested in my teaching" (Dieu-INT). One academic (Dang) expected to receive lower TT-SET results. She explained: "I lacked experience for teaching the course, and it was my first time" (Dang-INT). These expectations indicate that the academics evaluated the effectiveness of their changes through judgment of their effort, experience, and observation of students' attitude and expectation.

\subsubsection{POT as possible promoter of reflection}

Participants were divided in their perception of how peer feedback and observation of peer's teaching were useful for reflecting on and informing practice, as described in Table 6.5.

Seven academics regarded peer feedback as useful or reliable for reflecting on practice that could lead to learning new teaching ideas. The perception emerged from the perceived qualities of the peer such as: pedagogical knowledge, constructive feedback, good ideas, experience, and appropriate and precise feedback. For example, Hang evaluated her peer's feedback: "Nga's feedback was useful for me because of her experience and pedagogical knowledge" (Hang-INT). Teaching dialogue helped academics identify teaching aspects that needed improving. For example, Binh said: "Her [Cuc's] feedback helped me solve my problems and make changes to teaching" (Binh-INT). 
Table 6.5: Perceived values of peer feedback and classroom observations

\begin{tabular}{|c|c|c|c|c|}
\hline \multirow[t]{2}{*}{ Participants } & \multicolumn{2}{|c|}{ Peer feedback } & \multicolumn{2}{|c|}{ Observing a peer's teaching } \\
\hline & Perceived value & Reason & Perceived value & Reason \\
\hline \multicolumn{5}{|c|}{ Positive perceived values } \\
\hline En & useful & good ideas & useful & good techniques \\
\hline Dang & useful & exact, appropriate & useful & good techniques \\
\hline Dieu & useful & good ideas & useful & used for reflection \\
\hline Hang & useful & $\begin{array}{l}\text { experienced, } \\
\text { pedagogically sound }\end{array}$ & useful & good techniques \\
\hline Khoa & useful & $\begin{array}{l}\text { knowledgeable, } \\
\text { precise, constructive }\end{array}$ & useful & good techniques \\
\hline \multicolumn{5}{|c|}{ Some positive perceived values } \\
\hline Cuc & not useful & non-critical & useful & used for reflection \\
\hline Giang & useful & $\begin{array}{l}\text { peer young, } \\
\text { inexperienced }\end{array}$ & normal & $\begin{array}{l}\text { less skilled, similar } \\
\text { techniques }\end{array}$ \\
\hline Nga & not useful & $\begin{array}{l}\text { non-critical, } \\
\text { inexperienced }\end{array}$ & useful & used for reflection \\
\hline \multicolumn{5}{|c|}{ Both positive and negative perceived values } \\
\hline \multirow[t]{2}{*}{ Binh } & $\begin{array}{l}\text { not useful, } \\
\text { discouraging }\end{array}$ & peer judgmental & no opinion & no opinion \\
\hline & useful & $\begin{array}{l}\text { knowledgeable, } \\
\text { constructive }\end{array}$ & useful & $\begin{array}{l}\text { specialized } \\
\text { knowledge }\end{array}$ \\
\hline Phung & $\begin{array}{l}\text { useful, } \\
\text { discouraging }\end{array}$ & negative & useful & $\begin{array}{l}\text { good techniques, } \\
\text { used for reflection }\end{array}$ \\
\hline \multicolumn{5}{|c|}{ Negative perceived values } \\
\hline An & not trustful & $\begin{array}{l}\text { peer young, } \\
\text { inexperienced }\end{array}$ & not useful & $\begin{array}{l}\text { inexperienced, } \\
\text { young }\end{array}$ \\
\hline
\end{tabular}

Although there were four academics' concerns that peer feedback was unhelpful or non-critical or the peer was young and inexperienced in teaching (see section 6.3.2.2), three of them still considered observation of the peer's teaching as useful for reflection. For example, Nga explained why the experience of observing the peer's teaching was useful:

I expected Hang to figure out my weaknesses, but she did not. There must have been teaching aspects that needed improving. It is a pity... However, I could learn something observing Hang's teaching by analysing why some of her techniques were not effective and then did differently in my class. For example, in teaching new words in a reading passage, instead of explaining their meaning deductively as Hang did, I let students interpret their meaning by eliciting them from different contexts. $(\mathrm{Nga}-\mathrm{INT})$

In general, reciprocal POT facilitated observational learning whereby academics observed their peer's teaching behaviours and then used the experience to guide their actions. Nine academics considered observing a peer teach as useful because they could learn good teaching techniques or use the peer's teaching as a lens for reflection. 
For example, Binh said: "I learned a lot from observing Cuc teach. For example, she delivered a creatively and interestingly organized presentation of a difficult lesson" (Binh-INT).

In short, the degree to which participants believed POT was good for their reflection and learning appeared to depend on their perception of the criticality of peer feedback, the relevance and depth of their peer's feedback, their peer's attitude of feedback, teaching capacity and experience. For the academics (such as An, Binh, Hang, Nga, Cuc, Giang) whose age and experience was much different from their peers, their perception of POT may be associated with their positioning of themselves in comparison with their peer status and the collaboration and collegiality between peers. This kind of positionality in interpersonal relationships appears to impact on academics' interpretation of POT experience associated with their evaluation of their peer's quality of feedback, the ability to give critical feedback, and teaching capacity. Insights into academics' perception of POT with regard to the peer relationship are more fully reported in the section that looks at possible challenges to the implementation of TT-SET augmented with POT (see section 6.3).

\subsubsection{TT-SET plus POT: promoting reflection through structured steps}

The intervention process involved academics in steps such as targeting teaching aspects for improvement, planning observations, reciprocally observing teaching, debriefing with their peer, and writing reflection reports. All the steps can be seen as contributing to academics' reflection. Academics perceived the structured steps, including TT-SET, reciprocal observation of peer teaching, debriefing with peers, and reporting through the teachers' reflection report form, promoted reflection. The finding was supported in two ways: (i) the participants valued all the steps involved in the intervention as a whole, and (ii) they also indicated particular steps they thought most contributed to their reflection, which implied that these steps all contributed to academics' decision making.

Participants' reflection was evident in the structured procedure of implementing TTSET augmented with POT. Participants systematically identified problems of practice within actual teaching contexts from early-term TT-SET and POT. They were provided with clearly proposed steps in the process to support reciprocal POT such as 
facilitating collaborative dialogue about teaching after POT and proposed timeframes. They also participated in the development of tools such as TT-SET and POT protocol (pre-observation form, peer observer's feedback form, and teacher's reflection report form). Then, the exercise of debriefing and end-of-term interview helped academics make their pedagogical reasoning explicit. For example:

- identifying problems of practice in their teaching context from TT-SET and their peer: "TT-SET is supportive to my teaching in that it guides me to adjust my teaching appropriately" (Dang-INT). "I recognize that I need to promote the aspects which my peer highly valued and make changes to what really needs improving" (En-TRR2).

- engaging in dialogue about teaching: "Debriefing is very useful with dialogue about teaching where I could hear explanations on teaching aspects” (Binh-INT).

- observing a peer's teaching: "In collaboration with Nga, I learned much from observing her teaching" (Hang-INT)

- evaluating present practice against their past practice based on TT-SET, peer feedback, and observation of their peer's teaching by writing up their reflection: "Writing reflection reports is useful because it follows other steps systematically" (Dang-INT).

Through the structured steps, academics articulated their beliefs and tacit knowledge and made changes to current practice or planned changes to future practice:

- articulating their beliefs and tacit knowledge: "In the past, I just stuck to the textbook content, but now I am more flexible and selective in teaching (Hang-INT).

- changing current practice and planning to change future practice: "I changed by promoting students' communication in English through meaningful tasks" (Dieu-INT). "I will make lessons more interesting and reachable by relating them to the reality" (Dieu-TRR1).

Most academics valued all the steps as a whole. However, in answering the question regarding which steps in the intervention process were particularly useful to academics' reflection and learning about teaching, participants indicated particular 
steps that most contributed, although they said that the whole process was useful (Table 6.6).

Table 6.6: Usefulness of steps in the current intervention for reflection and learning

\begin{tabular}{|l|l|l|l|l|l|}
\hline Participants & $\begin{array}{l}\text { Collecting } \\
\text { TT-SET }\end{array}$ & $\begin{array}{l}\text { Pre- } \\
\text { observation } \\
\text { meeting }\end{array}$ & $\begin{array}{l}\text { Doing } \\
\text { reciprocal } \\
\text { observation }\end{array}$ & $\begin{array}{l}\text { Post- } \\
\text { observation } \\
\text { meeting }\end{array}$ & $\begin{array}{l}\text { Writing } \\
\text { reflection } \\
\text { reports }\end{array}$ \\
\hline Binh & & & & & \\
\hline Cuc & & & & & \\
\hline Dieu & & & & & \\
\hline En & & & & & \\
\hline Phung & & & & & \\
\hline Giang & & & & & \\
\hline Hang & & & & & \\
\hline Nga & & & & & \\
\hline Dang & & & & & \\
\hline Khoa & & & & & \\
\hline
\end{tabular}

Table 6.6 indicates that in their perception of which steps contributed to their learning, academics put weight on collecting TT-SET, doing reciprocal observation, and debriefing. Counting the comments through content analysis shows that eight participants supported the usefulness of TT-SET, seven participants supporting debriefing, and five supporting doing reciprocal observation. Particularly, Dieu, Nga, Dang shared a common view that all the steps are useful for reflection. For example, Nga said: "All the steps in the process influenced my decision-making” (Nga-TRR1). Other examples include interests in collecting early-term TT-SET and engaging in dialogue with their peer at the debriefing. Binh said: "Collecting TT-SET helped me gain understanding of student needs whereas talking to a colleague offered an opportunity to learn teaching ideas for better practice" (Binh-TRR1). Five academics valued collecting TT-SET and being observed by their peer. Khoa said: "Collecting student feedback and teaching observation are more important" (Khoa-INT). In addition, five academics (Dieu, Phung, Nga, Dang, and Khoa) also mentioned the usefulness of writing reflection reports. Dieu said: "Writing the reflection report is a useful way to consciously evaluate teaching" (Dieu-INT). Nga mentioned another aspect of teacher's reflection report: "It is useful for memorising what was reflected on" (Nga-INT). Dang viewed writing reflection reports as useful for reflection: "It [writing the teacher's reflection report] was useful for looking back on my performance." (Dang-INT). 
The role of the intervention on promoting academics' awareness or reflection on practice may be illustrated by what En and Dang wrote in their reports:

The programme was very useful for colleagues who are friends and want to support each other for development and teaching improvement. (En-TRR1)

In the process of teaching, if we continue to collect TT-SET and peer feedback like this programme, I will completely support it and join in. This activity allows me to know my students and gain understanding of myself and practice. (Dang-TRR1)

An did not appear in the table above because she contradicted herself. Although An did not have a positive experience of the intervention, she gave her evaluation of the process: "All steps systematically interact with one another, helping academics to draw out an overview of their teaching. The conversation between peers was important" (An-INT). An seemed to contradict herself in that on the one hand she did not value TT-SET or POT, on the other hand she considered the process as useful. This may mean she thought the intervention process itself to be useful or at least made her aware of teaching, but maybe not in this particular experience of it. In brief, the academics perceived several aspects of the process, or the overall process in some cases, as useful for their reflection. Reflection may be encouraged through these structured steps in the intervention process.

In summary, the academics perceived several aspects of the process of TT-SET augmented with POT, or the overall process in some cases, as useful for their reflection. In the process of pedagogical reasoning, TT-SET may provide academics with information about their teaching so that they could identify teaching problems or diagnosing their learning needs. TT-SET, reciprocal observation, debriefing, and writing reflection reports were perceived as important structured steps for reflection. These steps may support academics in choosing strategies to improve practice or evaluating the changes they made. POT provided a chance to learn from peer feedback and teaching observation where academics gained pedagogical knowledge: useful teaching techniques or strategies through dialogues and/or watching peers teach. Reporting through written forms may raise academics' consciousness in their evaluation of or reflection on practice. In short, these steps appear to contribute to academics' reflection and self-directed learning about teaching. 


\subsubsection{Improving the intervention to encourage participation}

With respect to improving the intervention to encourage participation, academics' suggestions focused on regulations for POT, policies that benefit academics, timing of POT, characteristics of the peer, and a professional group of practice.

Eight academics thought that POT should be voluntary. When people feel comfortable they will join in and learn about teaching. Khoa said:

POT should be voluntary. If it is imposed upon academics, they will not feel comfortable to participate. Those who have passion in teaching are willing to participate, so it is better to encourage them to join in POT. (Khoa-INT)

In contrast, three others suggested POT should be compulsory and set as academics' official responsibility. Cuc said: "POT should be regulated as academics' responsibility to make POT a routine. For example, they have to conduct POT for 50\% of their course time" (Cuc-INT). Nga gave a reason for compulsory POT: "Young [early-career] academics will volunteer to participate, but the more experienced ones will not. Thus, it is better to impose upon them" (Nga-INT).

Secondly, some of the academics that preferred voluntary POT suggested some form of incentives to encourage participation. Two participants were willing to participate in POT regardless of whether incentives should be given, but six participants suggested that academics should be offered incentives such as awards, gifts, titles, or pay to encourage their participation in POT. Nga said: "When deciding whether to join in POT, people may first consider if they will have benefits such as awards or pay" (Nga-INT).

Suggestions also related to the timing of POT. Nine academics perceived time as a challenge (see section 6.3.2.1) due to heavy workloads and the duration of the intervention being too short to see changes. Five academics accordingly suggested that the intervention should be conducted for a year (two semesters). Giang said: "The intervention process should be conducted over a long period of time so that teachers can make changes. Two semesters would be OK" (Giang-INT). Phung gave an opinion about scheduling academics' teaching time and the time between observations:

Academics' timetable should be conveniently arranged so that they can observe each other's class. The time between observations should be from 7 to 8 weeks 
so that we have more time to think deeply about our practice. Short time does not allow changes. (Phung-INT)

Academics described the characteristics of their preferred peer with respect to level of expertise or experience, relationship, and where they are from. Seven academics suggested that peers should be similarly experienced teachers or experts in the field. Nga suggested: "The peer should be someone that can give critical feedback. Thus, a dyad of peers should be composed of experienced academics for effective POT" (Nga-INT). Two academics preferred to collaborate with a peer who has similar experience (age). Binh said:

Those who have similar experience (years of teaching) or who are friends are willing (open) to share their teaching ideas. Thus, dyads for POT should be those being at about the same age. (Binh-INT)

Three mentioned that trusted peers are good for them. Giang said: "The effectiveness of POT depends on trusted peers" (Giang-INT).

Seven academics also gave their opinion about whether the peer should be an insider or outsider (a colleague inside or outside their institution). Five stated that they preferred an outsider observer. For example, Cuc said:

I prefer an outsider observer (e.g., a foreign expert) because they can give objective feedback. Their feedback will not be biased by the nature of relationship as that of the peers who are in the same institution. (Cuc-INT)

Two academics preferred an insider observer (colleague in the same department or institution). Phung said: "I preferred an insider observer because the person will know the context including students, programmes, and existing problems at the institutions to give appropriate suggestions for teaching improvement" (Phung-INT). Khoa preferred to collaborate with a colleague from a different discipline: "Observing teaching by a colleague from a different discipline can bring about good teaching ideas" (Khoa-INT).

Finally, there was a suggestion from Cuc and Hang that a seminar or forum should be established for academics of the department to share teaching ideas. Hang said: "After the course finishes, there should be a session where all academics can share their experience of what they did successfully and what challenges they had" (Hang-INT). 
In summary, in order to encourage participation in the intervention process, academics suggested that attention should be paid to whether or not POT should be voluntary and whether academics should be extrinsically motivated with incentives, appropriate timing POT to suit academics' schedule, pairing with their preferred peer, and a form of group learning.

\subsection{Challenges to the implementation}

Sub-question 3: What are possible challenges to the implementation of TT-SET augmented with POT in Vietnam's context?

The intervention faced several challenges, which were related to several academics' perception of TT-SET, and assumptions and experiences of POT.

\subsubsection{Perception of TT-SET as a challenge}

The implementation of TT-SET during the intervention faced challenges because of academics' caution or doubt about its reliability, leading to limited use or rejection of student feedback. Four academics (Dieu, Hang, Nga, and Khoa) stated that the results of SET required critical consideration before use because they perceived it as being distorted by factors including student carelessness, subjectivity, first impression, and lack of responsibility and wholeheartedness.

They believed that students might have given feedback with an "I-do-not-care" attitude, failing to provide accurate information and an entire picture of academics' teaching practice. Dieu said, "Many students just ticked on the TT-SET questionnaire automatically like a machine. They did not even think about it" (Dieu-INT). Perceiving that student feedback was superficial, Hang said, "I see that they preferred playing to studying. They act for their trivial personal desire" (Hang-INT). Therefore, they used student feedback with care. Hang said: "I consider TT-SET, but I need to think critically about whether it can truly reflect my teaching" (HangTRR1). Nga believed that first impressions might have altered students' view of academics' performance. Another challenge identified was that student expectations of teaching were different from academics' goals for effective teaching and learning. This mismatch could result in students' negative feedback on academic performance. Khoa mentioned that students would ignore an academic's teaching if it did not meet their expectations. Their perception resulted in limited use of TT-SET for reflection. 
An did not use TT-SET for reflection because she doubted its reliability and validity. In addition, An's rejection of the early-term TT-SET seemed to result from the misconception of its purpose and her confidence in her practice. Regarding the earlyterm SET as inaccurate, An ignored it and believed that she could teach well without paying attention to the feedback:

To tell the truth, I did not read the feedback carefully, just glanced at the figures...

I did not have time even though there were some explanations for the figures, and I do not care much about them... I always try my best in teaching. (An-INT)

Instead of using TT-SET, An believed that observing students' attitudes was a more accurate means to know if students were satisfied with teaching performance.

In general, academics are like policemen. Besides the (pedagogical) theories with which we are qualified, there is intuition or sensitivity: you can know whether students like you or not... If students get bored, I know that my teaching is not good. Yes, our observation or feeling is accurate. (An-INT)

Thus, An felt she did not need and trust TT-SET but could rely on her own eyes.

\subsubsection{Challenges to POT}

When asked about the challenges academics faced, they gave hypothetical challenges in addition to the ones they actually experienced during the intervention. Thus, this section presents possible challenges academics thought of and their actual challenges.

\subsubsection{Perception of potential challenges}

This section provides an analysis of academics' perspectives of potential challenges to the implementation of POT. During their interviews, academics speculated on eight potential challenges (see Table 6.7). 
Table 6.7: Potential perceived challenges to POT

\begin{tabular}{|c|c|c|c|c|c|c|c|c|c|c|c|c|}
\hline \multirow[t]{2}{*}{ Possible challenges } & \multicolumn{11}{|c|}{ Participants } & \multirow{2}{*}{ 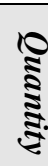 } \\
\hline & $\geqq$ & $\stackrel{\square}{\Xi}$ & 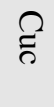 & $\underset{\mathscr{Q}}{\mathscr{Q}}$ & $\stackrel{T}{g}$ & $\underset{\sigma a}{\stackrel{D}{E}}$ & 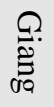 & 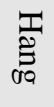 & $\underset{\alpha a}{\alpha}$ & 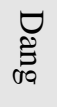 & 중 & \\
\hline A sense of discomfort & $\checkmark$ & $\checkmark$ & $\checkmark$ & $\checkmark$ & $\checkmark$ & $\checkmark$ & $\checkmark$ & $\checkmark$ & $\checkmark$ & & & 9 \\
\hline Poor colleague relationship & $\checkmark$ & & & & $\checkmark$ & & $\checkmark$ & $\checkmark$ & & & & 4 \\
\hline Insufficient skill for feedback & $\checkmark$ & $\checkmark$ & & $\checkmark$ & & $\checkmark$ & & & & & & 4 \\
\hline $\begin{array}{l}\text { Limited time for POT and } \\
\text { changes due to workload }\end{array}$ & & $\checkmark$ & & $\checkmark$ & $\checkmark$ & $\checkmark$ & $\checkmark$ & $\checkmark$ & $\checkmark$ & $\checkmark$ & $\checkmark$ & 9 \\
\hline Loss of financial benefits & $\checkmark$ & & $\checkmark$ & & & & & & $\checkmark$ & & $\checkmark$ & 4 \\
\hline No desire to learn from peers & & $\checkmark$ & & & $\checkmark$ & & & & & & & 2 \\
\hline $\begin{array}{l}\text { Non-tenured employment (Class } \\
\text { size and bad premises) }\end{array}$ & & & $\checkmark$ & & & & & & & & & 1 \\
\hline $\begin{array}{l}\text { Perfunctory action due to } \\
\text { observer effect }\end{array}$ & & $\checkmark$ & & & & & & & & & & 1 \\
\hline
\end{tabular}

The first challenge was a sense of discomfort. Nine academics perceived that POT could make them feel scared or uncomfortable with the presence of observers in the classroom. They believed that observations may disturb academic autonomy and make them feel nervous. Further, unfamiliarity with POT and fear of humiliation were articulated as contributing to the feeling:

Academics do not like POT because they love their own freedom in the classroom. People are still conservative. They just want to do what they like. They may use some 'tactics' which are not 'methodological' but interesting to students, and do not like others to see this aspect of their teaching. (Binh-INT)

The second challenge was the nature of colleague relationships. Poor colleague relationships were seen as detrimental to POT because of the belief that if colleagues were unwilling to learn from each other, feedback would trigger tension, resulting in ineffective POT:

If we do POT with people we do not like, it is hard to learn from them. Thus, POT is like torture rather than a chance to share ideas about teaching, especially when peers have completely different views of teaching effectiveness. POT values will be promoted if colleagues trust and work well with each other. (Hang-INT)

Also poor relationships might also put confidentiality at risk and cause possible humiliation. This would diminish the effectiveness of POT for cooperative learning about teaching. 
Not all colleagues who are good to each other. POT may be a chance for backstabbing. In this case, people do not really want to give useful advice to help their peer make progress, but try to criticise one's weaknesses before others. (EnINT)

Third, insufficient skills in giving and receiving feedback were also mentioned as a possible obstacle for POT. Four academics indicated that observers often had an attitude of nit-picking over tiny details, making observed academics uncomfortable:

The major point is that we need to see the core value of a teaching session: why do students like this academic? But the common thinking of POT is nit-picking over tiny details. Thus, POT should be carried out with instruction on the core value or bigger things. (An-INT)

The fourth challenge was limited time for POT and initiative for changes. Nine academics perceived that the facilitation of POT could be problematic because of the limited time available and the workload involved to observe other classes. Khoa said: "Academics that have heavy schedules will be unable to join in POT" (Khoa-INT). The schedule for POT may need to be considered so that the allotted time can facilitate changes. Dieu said:

Timing of POT should be considered. POT should come at the beginning or near the end of the course. Academics will be overloaded if they do POT during midterm because they have to prepare for midterm tests. (Dieu-INT)

Financial pressures was another challenge articulated by four academics. Because of low pay, academics had to work more to make money. Therefore, spending time on POT would affect academics' financial benefits. Khoa explained: "Academics have low salaries, so they do not want to do POT because they prefer teaching more to earn money instead" (Khoa-INT). Also, low pay may demotivate academics to initiate changes to teaching, so they would refuse to join in POT:

Participate in POT for changes? How can academics make changes when their pay is $50,000 \mathrm{VND} /$ hour [about less than $3 \mathrm{NZD}$ ]? (An-INT)

Another challenge mentioned by two academics was lack of a desire to learn from colleagues. One noted that:

Observing others' teaching makes observers sleepy to see the same thing or something they know recur. Just new things interest them. Thus, POT is useful 
for those who are active, enthusiastic, and willing to learn... If academics are not free to choose which colleague to observe, they will feel uneasy. (Binh-INT)

Next, teaching conditions were also discussed as an obstacle. One academic indicated that the classroom was overcrowded and lacked facilities, which might result in failure to achieve teaching effectiveness. Poor facilities could not facilitate effective teaching, and academics could be blamed for their personal incompetence rather than objective causes. This could deter non-tenured academics from participating in POT:

There could be opposition to compulsory POT. The grades for English are not added up to students' learning results. Students are willing to drop class, which puts visiting academics under pressure for fear of being judged "bad" based on the number of students present. The fear also results from the poor premises and crowded classroom. (Cuc-INT)

Finally, another potential challenge is observer effect that may lead to observed academics' perfunctory action. Binh thought that the observed teacher will only improve certain aspect of teaching due to the appearance of an observer. She said: "When teachers do not feel comfortable because of the pressure of observation, they may do it perfunctorily" (Binh-INT).

In summary, academics described eight possible challenges that might hinder their participation in POT as shown in Table 6.7. Most common were academics' perceived sense of discomfort, limited time available for POT and initiatives for changes, loss of financial benefits, insufficient skill for feedback, and poor colleague relationships.

\subsubsection{Actual experienced challenges}

This section provides an analysis of academics' actual experienced challenges to the implementation of POT in the university where the study was carried out.

\section{Perceived limited learning from the junior peer's lens}

The first barrier is senior academics' (i.e., those who have greater age, or greater age and experience) perceived limited learning from the junior peer's lens. This barrier is unique to senior-junior pairs such as Cuc-Binh and Nga-Hang. First, both Cuc and Nga perceived a lack of critical comments (e.g., negative feedback) from their junior peers Binh and Hang respectively. They thought deference existed in their feedback. Binh and Hang, five and nine years younger than their respective peers - Cuc and Nga were seen to be hesitant about giving critical feedback. For example, Cuc said: 
I do not highly value Binh's non-critical feedback, which was affected by the oriental culture with deference paid to the older and higher status. She must have known my age and status and was reluctant to give critical feedback for fear of losing face due to lack of experience or humiliating me. (Cuc-INT)

There were two different reasons behind the lack of negative comments from the junior peers: junior peer's deference and senior peer's good teaching. In the former case, Binh gave only positive comment because of the deference she offered to Cuc. Binh conveyed her deference indirectly:

In sharing ideas about teaching, when I see good things from Cuc, I will learn them. Otherwise, I will ignore her pedagogical approach which is not suitable for me. If two peers have the same teaching experience or age, or closeness in their relationship they are willing to be truthful with each other about teaching problems, e.g., suggesting their peer do this and that. If there is a distance in experience and age, will the junior peer dare to be truthful about the senior's problems? (Binh-INT)

The perceived deference in both Cuc and Binh implies that their deference may have hindered open critical dialogue about teaching. In this case, the challenge was perceived the same by both peers: Cuc perceived deference, and Binh paid deference to Cuc. In the latter case, Hang gave only positive comment to Nga because Hang saw Nga's teaching was so good that she did not see aspects that needed improving. Hang said: "I learned from her knowledge of the subject and pedagogical methods. She is like a teaching model" (Hang-INT). This means the junior thought her peer taught well and gave positive comments while the senior thought it was because of deference. Second, senior peers' limited learning from the peer's lens because they perceived their peer was not as experienced as they were in terms of teaching (i.e., they could not learn better teaching skills from observing the peer). Three senior peers Cuc, Nga, and Giang considered their peers Binh, Hang, and Phung as juniors. For example, Giang said: "I may have experienced what Phung is experiencing in her teaching now. I have already known every step she took" (Giang-INT).

Despite not receiving critical feedback from their junior peer, these senior peers (Cuc, Giang, Nga) perceived that they can learn from the junior peers in other ways. For example, Cuc expressed her opinion: "A debriefing may not be necessary. I think I just need to observe and learn from the peer's good aspects of teaching as well her 
problems" (Cuc-INT). Although they had freedom to select their peer, these academics still thought they would need a compatible peer. For instance, Nga suggested: "The pairing of peer should be in a way to promote critical feedback. It is mutual or collaborative reflection, so for it to be effective I may need a peer who is as experienced as I am.” (Nga-INT). In the same vein, Binh suggested: “The pairing of peers that are similar in experience or age would make sharing teaching ideas easier" (Binh-INT). The evidence indicates that the difference between peers in terms of experience or age, or both experience and organisational status (e.g., Cuc and Binh) generated a challenge, which somehow hindered open conversation about teaching. It also shows that that participants in these unequal pairings felt that more equal pairing would have facilitated more critical feedback.

\section{Disagreement with feedback and lack of perceived sensitivity}

The next challenge is the disagreement with feedback and lack of perceived sensitivity in giving and receiving feedback. This challenge is unique to the senior-junior pair AnBinh. Binh was paired with both Cuc and An. Comparing the way Cuc and An gave feedback to her, Binh perceived that An's feedback was judgmental.

Unlike An, Cuc had a conversation with me with her sensitivity. She was so kind to ask me if it was possible to try a different way of organising the class when she thought that my way was inappropriate. I was pleased with her feedback. In contrast, An wanted to give me feedback but she said I did this and that wrongly. Meanwhile, my students told me that they caught up with the lesson well. Nothing right or wrong here. So, I turned irritated. (Binh-INT)

Additionally, Binh perceived An showed her positioning during the post-POT dialogue, which prevented her from giving further feedback. She explained:

An said that she is older and more experienced than I am, so she wanted to share her teaching ideas with me. How could I dare to give feedback to her when she means I lack experience and I am too young [for her to learn something]? (BinhINT)

The evidence showed that Binh perceived An's attitude of 'superiority' and lack of respect for her. Binh also talked to the researcher after the debriefing, explaining why she became irritated: “I am not satisfied with An's feedback. It was not constructive and not focused on the areas I wanted. It was superficial and unrelated to teaching” $(\mathrm{RJ})$. 
At the debriefing, in response to An's feedback, Binh gave feedback to An in a way that made An irritated (as evidenced in the following quote). An, 16 years older than Binh, showed her disagreement and anger:

I have never looked down on students in my life. I often give students both positive and negative feedback on their presentations. Binh said I scolded them; this is not true. Early-career academics like her will not, I think, probably have an ability to give feedback on my teaching. (An-INT)

Binh's reaction may have made An feel that the junior peer was challenging her values. In the interview, Binh confessed that she had behaved inappropriately: "When I thought An's feedback was not appropriate, I felt irritated and criticised her teaching. Recalling this instance, I think I behaved incorrectly" (Binh-INT). The evidence shows that tension arose when there was disagreement with the peer's comment on teaching and the lack of perceived sensitivity about giving and receiving feedback. Both An and Binh expected to have a compatible peer with respect to experience and age. For example, An said: "My peer is too young and lacks experience in teaching... If my peer had been an experienced one, I would have learnt much from her feedback" (An-INT). The lack of sensitivity in dealing with disagreement in giving feedback brought about both peers' negative feeling and defensiveness, resulting in a poor peer relationship. Binh said, "I felt irritated by An's feedback which affected our relationship" (Binh-INT).

Besides, giving only negative comments on peer's teaching can be discouraging. For example, Phung said: "Giang gave only negative comments on my teaching. I felt discouraged but I thought she was truthful about it and accepted them as an opportunity to improve teaching" (Phung-INT). This may have caused Phung a bad feeling although she dealt with it.

This challenge presents a complicated issue of ways of giving and receiving feedback. It may have been the combination of the peers' respective personalities (a sensitive issue) which may be influenced by the peer's perception of their position regarding age and experience. Also, the imbalance between negative and positive comments may cause the peer negative feeling. 


\section{Limited time for POT and changes}

Limited time was perceived as challenging for academics to participate in POT and take initiative for changes. Although nine academics thought that limited time was a challenge, four (Binh, Giang, Hang, Nga) actually found it difficult to participate in POT because of their workload and limited time available to observe other classes and to make changes:

To be honest, observing others' teaching is mostly impossible for me even though I know POT was effective for improving teaching. This time, I joined in POT just to help you with your project. I felt tired with a heavy workload. (Giang-INT) I felt uncomfortable during the POT periods because I had to complete my research and joined in POT in another university. I was under pressure, so I just performed perfunctory teaching. (Binh-INT)

Thus, the lack of time resource due to academics' heavy workload or tight schedule appeared to be a barrier to the implementation of POT.

\subsubsection{Comparing potential and actual perceived challenges}

Comparing academics' speculated potential challenges and their actual ones, it is easy to realise that limited time for POT and changes was both anticipated and experienced challenge. The other two anticipated challenges were associated with difference in age and experience. The actual challenges of disagreement with feedback and lack of perceived sensitivity were somehow related to poor peer relationship, which was distant due to differences in age and experience. Four participants thought that poor peer relationship could be a challenge to POT, and what actually happened was a breakdown in a pair's relationship due to perceived criticism and perceived lack of respect. Perceived limited learning from the junior peer's lens was an actual challenge which was not speculated among potential challenges. Junior's deference and inexperienced teaching were perceived by academics. Thus, in addition to the time issue, the nature of the peer relationship could be a cultural issue to be discussed later.

\subsubsection{Summary}

This section identified several actual challenges to the intervention that hindered academics from reflection and learning about teaching. The barrier to academics' use of TT-SET is their perception that it was a blunt instrument to inform teaching because 
it did not truly reflect what students needed and included distorted information. This perception may be impacted by academics' misconception of its purposes. The barriers to POT include limited learning from the junior peer's lens, disagreement with feedback and lack of sensitivity in giving and receiving feedback, and limited time for POT and changes.

\subsection{Changes in academics' pedagogical reasoning}

The main question: In the context of a Vietnamese university, how does TT-SET augmented with POT impact on academics' pedagogical reasoning?

This section addresses the main question and the sub-questions, based on evidence presented in previous sections.

\subsubsection{Enhanced reflection and new comprehensions}

Finding 1: The intervention promoted most academics' reflection which led to their learning of new strategies to refine practice or changing of teaching assumptions.

- The academics perceived several aspects of the process (e.g., TT-SET, reciprocal observations, debriefing, and writing reflection reports), or the overall process in some cases, as useful for their reflection. Reflection can be the what, the how, and/or the why of teaching.

- Academic perception of the quality of TT-SET, and peer feedback and peer's teaching in the POT process determines their use of the gathered data for reflection to improve teaching: (1) Academics who thought information they received through TT-SET and POT was reliable and useful took it into consideration for reflection and to improve their teaching; (2) Academics who believed that the quality of TT-SET and POT was distorted and limited used student and peer lenses in a limited way; (3) When academics did not trust students or peers, they did not use their feedback as guidance for changing their practice. However, even when they did not think their peer's feedback was particularly valuable, they learnt from the observation experience to make changes.

Sub-finding 1.1: The intervention enhanced academics' awareness of themselves including their role of an English language teacher, their position or status in relation to their peer, and professional identity through writing reflection reports or having teaching dialogue with their peer. 
Sub-finding 1.2: The intervention facilitated the academics' articulation of teaching and learning philosophies through writing reflection reports or having teaching dialogue with their peer.

Sub-finding 1.3: The nature of the peer relationship impacted on the extent to which academics responded to peer feedback for reflection to improve teaching and the perceived value of classroom visits:

- Academics' positioning of themselves (in terms of age, experience, organisational rank, and expertise) regarding their peer's status impacted their interpretation of POT experience. When there was a great distance in the relationship between peers (e.g., age and experience), limited collegiality resulted due to junior peers' deference. This hindered critical open dialogue between peers.

- When peers do not have a close relationship, judgmental attitudes (which is harmful to respect and face saving) may cause a breakdown in the relationship. This is associated with the lack of sensitivity in giving feedback.

- In many cases, sharing ideas about teaching and observing peers teach nurtured collegiality and collaboration between peers, except in some cases.

Sub-finding 1.4: The intervention process enhanced eight academics' single-loop learning (refining their practice) and, for three, double-loop learning (rethinking and restructuring their assumptions about teaching):

- TT-SET was regarded by many as a useful tool that facilitated academics' selfdirected learning because it allowed them to target teaching aspects for feedback and to act on them for improvement.

- POT provided a chance to learn from peer's feedback and teaching (through dialogue and written feedback and/or watching peers teach) where academics gained pedagogical knowledge such as useful teaching techniques or strategies.

\subsubsection{Perceived changes and future plans}

Finding 2: The academics' new comprehensions resulting from reflection can trigger academics' action for solving problems of current and future practice with an aim to improve teaching. 
- Ten participants took actions for changes which centred on technical and pedagogical aspects.

- Through the intervention, all participants set plans or aspirations for future practice. It should be noted that although An did not have a positive experience of the process, she became aware of her practice and intended to take actions for future practice.

\subsubsection{Enhanced confidence, self-efficacy and autonomy}

Finding 3: Ten participants reported increased confidence and many of them developed a sense of self-efficacy through their new understandings of their teaching and actions to refine practice.

- Through the intervention, academics developed their confidence in teaching and their belief in their capabilities to influence students' learning.

- TT-SET augmented with POT brought about mastery experience, vicarious experience, and verbal persuasion which were the possible sources of selfefficacy.

Finding 4: The intervention also appears to contribute to nine academics' sense of autonomy because it provided an opportunity for them to take control of their practice such as identifying teaching aspects in need for improvement.

- The steps of the intervention appeared to contribute to academics' self-directed learning.

- TT-SET was mostly regarded as a useful tool that facilitated academics' selfdirected learning because it allowed them to target teaching aspects for feedback and to act on them for improvement.

\subsubsection{Enhanced collaboration and collegiality}

Finding 5: Eight participants reported that POT made their peer relationships develop to a new level, promoting collegiality and collaboration.

- The development of a collegial relationship can be viewed as the willingness to share teaching ideas and beyond, modelling teaching, sharing of teaching materials, and mutual understanding. 
- Spending time together made the peer relationship develop to a new level (e.g., enhanced mutual understanding), promoting collaboration and collegiality.

- Collaboration and collegiality were nurtured when academics knew how to deal with sensitive aspects in the peer relationship, regarding respect for each other.

- Collaboration and collegiality were enhanced, particularly when peers were close friends.

\subsubsection{Actual challenges and possible solutions}

Finding 6: Academics' caution or doubt about TT-SET reliability was a challenge for its implementation, leading to limited use or rejection of student feedback. POT faced three actual challenges, including (1) perceived limited learning from the junior peer's lens; (2) disagreement with feedback and lack of perceived sensitivity; (3) limited time for POT and changes.

- The difference between peers in terms of experience or age, or both experience and organisational status somehow hindered open conversation about teaching. Also, participants in three unequal pairings felt that more equal pairing would have facilitated more critical feedback.

- Disagreement with feedback in combination with a lack of respect (a sensitive issue) may cause tension in the peer relationship.

- The lack of time resource due to academics' heavy workload or tight schedule appeared to make it hard for them to engage in POT and make changes.

Finding 7: Regarding solutions for attracting participation in the intervention, academics suggested that attention should be paid to whether or not POT should be voluntary and whether academics should be extrinsically motivated with incentives, appropriate timing POT to suit academics' schedule, and pairing with their preferred peer.

\subsection{Summary}

The intervention engaged most participants in a path to their professional learning through reflection and knowledge gain, initiatives for change, a sense of self-efficacy and autonomy, and building a strong relationship between colleagues. However, the way they reflected on teaching and gained a new understanding of practice and took 
actions resulted in distinct learning outcomes for each individual. For some, there was single-loop learning where participants refined or improved their practice by adjusting their own strategies or learning new strategies within their existing teaching beliefs. For a few, there was double-loop learning where participants rethought and restructured their assumptions about teaching. The intervention created an opportunity for changes based on reflection through structured steps as a whole. Academics' reflection and learning about teaching were subject to several enabling factors and challenges which were associated with their perception of the value of TT-SET, and POT including peer's feedback (criticality, agreement, and balance of negative and positive comments) and observation of peer's teaching, and time resource for POT. Academics' perception of peer's feedback and teaching was dependent on the nature of the peer relationship. 



\section{Chapter 7. Discussion of Findings}

\subsection{Overview}

This chapter discusses the findings from the implementation of TT-SET augmented with POT in a Vietnamese university context. It begins with a discussion of the research findings concerning participants' perceptions of how the intervention impacted on their pedagogical reasoning. It was perceived to have made them reflect on practice. Reflection is highlighted as the fundamental factor underlying changes such as academics' new comprehensions, changes to current practice and planned changes to future practice, enhanced confidence and in some cases self-efficacy. Ways in which the intervention appears to have brought about a sense of autonomy and promoted collegiality in many academics are also discussed, as are the nature of challenges which would need to be addressed for a successful intervention. Finally, a revised theoretical framework for analysing teachers' pedagogical reasoning in the Vietnamese context is outlined as a contribution of the study.

\subsection{Promotion of reflection}

The study shows that reflection can be promoted through a structured process embedded with formative information through student and colleague lenses as contexts for learning. In the setting of this Vietnamese university where academics teach solitarily and lack time to critically examine their practice, the finding is particularly important as it suggests a mechanism to support them in enhancing practice and therefore contribute to the literature in this context. Most academics' reflection led to their learning of new strategies to refine practice or changing of teaching assumptions, which in some cases appears to have triggered their actions to change practice. It also appears to have enhanced academics' confidence, in some cases self-efficacy, autonomy, and collegiality.

\subsubsection{Features that promote reflection}

The study shows that reflection was promoted through a structured intervention embedded with formative information from student and colleague as contexts for learning. The academics perceived the structured steps as useful for their reflection. They valued all the steps involved as a whole, which implies that the overall process 
contributed to academics' decision making, but academics also indicated there were steps which they felt had most impact.

First of all, TT-SET was a structured step that helped academics collect student feedback systematically. TT-SET prioritised what the academics needed to find out about because it is a form of formative SETs which focused on academics' needs (Winchester \& Winchester, 2011) and helped "identify areas where there is scope for improvement" (Hounsell, 2009, p. 198). TT-SET used in this present study provided academics with information on targeted aspects of teaching and stimulated their teaching aspirations (i.e., for a change and improvement) from identifying teaching problems. This finding aligns with several studies such as Winchester and Winchester (2011) and Aultman (2006) which used weekly and early-term formative SETs. For example, the use of TT-SET had a similar result to Aultman's use of early-term SETs in that the participants learned about students' expectations and preferred way of academics' teaching that contributed to their reflection and decision on making changes. Although there were in several cases concerns about TT-SET reliability, TTSET was perceived as a source of information on teaching to be considered. It can be argued that TT-SET may not be necessary because observing students' reactions or attitudes, the effective use of which may not be refuted, is enough for making changes. However, it is possible to say that TT-SET focused on the academics' needs and aspirations for practice and provided them with formative information that they could use to reflect systematically. TT-SET was designed to be complemented by other steps in POT.

Undertaking reciprocal observations helped academics focus on their practice in two ways: learning new teaching ideas from their peer or learning from their peer's teaching problems. Seven found observing their peer's teaching useful because they could learn new teaching ideas, whereas the others said they could not learn new ideas but recognised their peer's teaching problems and referred to their own situation. This finding replicates the finding from other studies that teachers can obtain new teaching ideas from observing their peer (Bell \& Mladenovic, 2008; Finn et al., 2011; Hendry et al., 2013; Hendry \& Oliver, 2012). For example, Bell and Mladenovic (2008) discussed in a study on POT partnership for tutor development that observing a peer teaching was a key benefit and appeared to be more highly regarded than peer feedback because it could provide tutors with new ideas and help them reflect on their teaching. 
Although there may be differences in the ways POT is implemented in studies such as Barnard et al., 2011; Cairns, Bissell, \& Bovill, 2013; Cosh, 1999; HammersleyFletcher \& Orsmond, 2005; Peel, 2005; and Bell \& Mladenovic; 2008, the common finding is that POT develops reflection and reflective practice. Watching how the lesson was delivered, how students engaged and reacted in the peer's classroom helped the academics perceive what strategies worked and are worth trying (Hendry et al., 2013; Moore et al., 2007). This finding also aligns with Bandura's (1977b) social learning theory that people learn within a social context, and that learning is facilitated through processes such as modelling, observational learning, and imitation. Thus, it is evidenced that reflection can be triggered by the nature of observation of a peer's teaching.

The debriefing session also contributed to academics' awareness of their teaching. Seven academics reported that they could learn from the debriefing session whereby they had discussion about teaching based on TT-SET and the observation. The debriefing session facilitated academics' sharing of teaching experience made explicit through observation (Atkinson \& Bolt, 2010; Carroll \& O'Loughlin, 2014). Peer feedback can complement student feedback because academics provide a different viewpoint (Hutchings, 1996). Although there was evidence that the teaching dialogue can promote learning, there is an argument about whether teachers talking about their teaching can produce new learning (Gosling, 2014; Palmer, 1998). Palmer argued that when a talk has "nothing more than 'tips, tricks and techniques'," it "fails to touch the heart of the teacher's experience" (p. 11). It seemed that most the pairs' debriefings focused on content and process reflection. Thus, in order to encourage academics to engage in deeper learning, it may be necessary to stimulate their premise reflection (e.g., peers can elicit each other to reflect more deeply on practice through thoughtprovoking questions and giving evidence gathered from observations). POT in this study is reciprocal, which means it created spaces for mutual or reciprocal learning. POT was structured to promote peers sharing teaching ideas and reflection. It is argued that the principal role of the peer observer in collaborative POT is framed not as a knower who provides solutions but as a supportive colleague who supports academics' reflection by describing observations, offering feedback and asking questions (Barnard et al., 2011; Gosling, 2014; McGill \& Beaty, 1995) when the peer relationship went well with positive learning experience and in a comfortable atmosphere. Reciprocity 
of learning is either of the two principles (i.e., parity and reciprocity) of collaborative POT Gosling suggested. As evidenced in chapters 5 and 6, although there were concerns about the peer's feedback quality and teaching skills or experience in pairs composed of senior and junior academics, many participants perceived that POT helped them focus on their practice. POT can help teachers gain insight into their pedagogy, curriculum, students, and themselves (Pressick-Kilborn \& te Riele, 2008; Sullivan et al., 2012). The finding concurs with Korthagen's (2010) idea that peersupported learning promotes collaboration and exchanges between colleagues. Korthagen (2010) believed that learning to teach is "a socio-cultural process relying on discursive resources" (p. 104). Therefore, teaching dialogue between peers was supportive of their reflection.

Writing reflection reports was viewed as a way to look back on teaching. Five academics valued writing their reflection after POT because it was useful for consolidating what was reflected on and another way to consciously think about their practice. This finding is also supported by evidence from Donnelly's (2007) report that "in writing reflectively about the experience you were subconsciously trying to figure out why you would do this and whether you were doing it better" (p. 124). It was also found in Bell's (2001) structured teaching development programme employing collaborative POT and educational developer, that the participants reflected on the POT experience in writing. This is what Fullerton (2003) described as the fourth stage of POT which consolidates the main points of the teaching conversation, responses to the peer's written feedback, and plans for future practice Thus, writing reflection reports appeared to be a systematic way of reflecting on the POT experience.

Although academics had different perception about steps (e.g., TT-SET, reciprocal observations, debriefing, and writing reflection reports) which they felt had most impact on their reflection and learning, they valued the whole process. This finding is again important for academics' changes in pedagogical reasoning because in an environment like this university where academics are often overwhelmed with workloads and teach in isolation, such intervention stimulated their reflective thinking and made them become aware of practice. These lenses appear to be structural aids to reflection as LaBoskey (1993) suggested. 


\subsubsection{Kinds of reflection}

Participants in this study varied in the types of reflection they conducted: content, process, and premise reflection. For seven academics, reflection was about problemframing and problem-solving (see Table 6.1). They focused on aspects of the what and the how of teaching. Several participants were more pedagogically aware of their teaching and content and process of others' teaching than TT-SET had alerted them to. For example, Binh, Hang, Khoa believed that they learned more from their peer than their students. This aspect of the finding replicates the finding by Cairns et al. (2013) that the POT process led academics to insights into others' content and process of teaching. Focusing on the what and the how of practice seemed common among many participants.

The findings indicated that three participants engaged in premise reflection. They reflected on the premise by questioning their teaching beliefs and assumptions (see section 6.1.1). This finding is also supported by the literature on POT. With respect to professional conversation about teaching between peers, POT facilitated rethinking their tacit knowledge and questioning their ways of doing things (Schuck et al., 2008) or challenged their preconceptions, making them more pedagogically aware (Harper \& Nicolson, 2013), illuminating the why of teaching (Donnelly, 2007). As Peel (2005) argued, premise reflection "demands an active engagement with abstract pedagogical theory, purposeful critical reflection on classroom practice, and a challenging of assumptions through shared critical reflection with colleagues, during both formal and informal conversations about teaching" (p. 494). These academics explained why they changed their teaching beliefs and assumptions. Dieu learned why reflection from students' and peer's feedback is important for changes to practice. Nga, by getting the evidence for her reasoning from her own practice and observations of peer teaching, reflected on why her choice of teaching methods worked and she became more critical. Khoa learned why she should direct students to learn rather than choose methods to fit their preferences. These academics went further than teaching techniques or teaching aids, and these examples illuminate the 'why' of teaching or premise reflection.

With the evidence supporting academics' reflection on teaching, it can be said that the intervention made academics focus on or be more aware of their practice. Reflection appeared to contribute to their learning about teaching. 


\subsubsection{What reflection promoted}

\subsubsection{New comprehensions}

TT-SET augmented with POT provided meaningful learning opportunities for academics in this study (see section 6.1). They gained new understandings of practice (e.g., new teaching ideas or pedagogical content knowledge, students' preferred way of teaching, and reflective abilities). These aspects of learning were evident in academics' reflection reports after POTs, in dialogue at post-POT debriefing, and interviews. This outcome replicates the findings from the literature that academics appreciated feedback and regarded POT as an opportunity for deep understanding and reflection (Cairns et al., 2013; Donnelly, 2007; Pressick-Kilborn \& te Riele, 2008; Shortland, 2004, 2010; Sullivan et al., 2012). For many, enhanced collegiality through POT (see Section 7.2.3.4) seemed to contribute to their learning because POT carried out with mutual trust and respect facilitated academics' learning and teaching improvement (Bell, 2005; Bell \& Cooper, 2013; Shortland, 2010). Academics' reflection from students and colleagues' lenses played a key role in their learning about teaching; however, the levels of learning were varied among the participants.

Learning could occur at 'gestalt formation' where academics' action tendencies are based on previous experience with similar situations by which their needs, thoughts, feelings, values, and meanings are unconsciously stimulated (Korthagen \& Lagerwerf, 2001). As an example of this level of learning, An with over 20 years' experience of teaching preferred to use "chalks and the blackboard" for teaching delivery. After observing the teaching of her peer who used PowerPoint for lesson delivery, An intended to use PowerPoint for her lesson presentation the following semester despite saying she did not learn from her peer. It seems that her need to use technical aids was stimulated by her comparison between observation experience and her previous experience. Although An did not make any changes to her current practice, her intention to change future practice indicates that she became aware of her practice. This is an example of implicit learning through what can be called "the development of awareness" (Korthagen, 2010, p. 101; Marton \& Booth, 1997). It means that the academic "has become capable of discerning aspects of the phenomenon other than those she had been capable of discerning before" (Marton \& Booth, 1997, p. 142). The development of awareness may contribute to future changes to teaching. 
Most participants' learning was at the 'schematisation' level whereby conscious actions are taken with a need for clarifying a situation and whereby concepts, characteristics, and principles of practice may be developed (Korthagen \& Lagerwerf, 2001). The academics reflected on their teaching and developed a conscious network of characteristics (e.g., of students), teaching principles, and concepts related to teaching strategies to describe problems in practice and how to solve them (i.e., how to act effectively when confronting similar situations). For example, many academics such as Dang and Phung realised that students liked to learn with fun, so their strategies to engage students in learning included fun activities such as games and role-play. It can be said that recognising students' preferred way of teaching and delivering teaching in a way to meet their expectations does not always necessarily make teaching more effective or improved, but may make students receptive to academics' teaching approaches. These academics' schemata, as Korthagen (2010) discussed, "are very much coloured by the desire to know how to act in particular situations" (p. 102). It appeared that POT created what was called contextual learning that promotes a collaborative teaching environment (Bell, 2001; Long \& Hoa, 2010; Potter et al., 2011; Shortland, 2010). As Knowles et al. (2005) discussed, "adults have a readiness to learn those things that they need to know in order to cope effectively with real-life situations" (p. 72). When participants learned new strategies and applied them to their classroom practice, they engaged in single-loop learning (Argyris \& Schön, 1974). There may be some possible explanations for many academics' single-loop learning. With reference to van Manen's (1977) levels of reflection, four academics (Binh, Hang, Phung, and Dang) can be seen as young academics who carried out reflection at a technical level because they may lack schemata in solving problems (Taggart \& Wilson, 2005). Hence they might have considered reflection as a way to gain knowledge and aimed to improve their practice in their particular context. It is easier to promote technical-practical reflection than critical reflection (Sparks-Langer \& Colton, 1991). As for the more experienced academics (An, Cuc, En, and Giang), they might have established their personal theories of teaching and learning and just tested what worked and how it worked with their theories.

Most importantly, learning occurred at the 'theory building' level where academics form the logical structuring and reassessing of the relations of the elements in the schema or several schemata that are connected into one coherent theory (Korthagen \& 
Lagerwerf, 2001). Through premise reflection, three academics (Dieu, Nga, Khoa) restructured their theory of teaching. For example, both Dieu and Nga had a common change in their thinking that becoming an effective teacher requires their engagement in critical reflection with student and peer lenses and ongoing changes. For Khoa, before she thought giving whatever students liked (e.g., fun lessons) was effective teaching, but after the intervention she realised that delivering so-called fun lessons did not aid to student learning, rather she delivered content that she thought was useful for student learning. Thus, these academics were building their personal theory of teaching, restructuring their teaching assumptions. This is what Argyris and Schön (1974) call double-loop learning where there was the changing of academics' assumptions about teaching. Not many academics reached the theory level because, as Korthagen (2010) explained: "The theory level is aimed at deep and generalized understanding of a variety of similar situations, whereas practitioners are often focusing on directions for taking action in a particular situation, and as a consequence, often do not reach the theory level" (p. 102). From interview data, the three academics said they deliberately thought about their practice in different teaching contexts and were open to different viewpoints of teaching to reach their desired outcomes. It suggests that these academics at least had three common attitudes towards their practice: whole-heartedness, open-mindedness, and responsibility, the necessary attitudes of a reflective teacher (Dewey, 1997).

In discussion on situated learning theory and the pedagogy of teacher education, Korthagen (2010) mentions a state of learning called "level reduction" (p. 103) whereby schematised or even theoretical knowledge becomes so self-evident that it can be used in a less conscious way as if it is reduced to one gestalt. This reduction allows more focus on other things as Korthagen (2010) claimed; the expert level in professional growth is "the level at which the professional can act fluidly on the basis of an intuitive grasp of the situation" (p. 103). Korthagen explained that teaching is a gestalt-driven activity, so developing adequate schemata in teachers requires sufficient fruitful practical experiences (whereby gestalts are developed) and promotion of reflection. While such level reduction was not observed in this study, the intervention engaged academics in contextual practical experiences and promoted their reflection. If it is carried out as an ongoing process, the schemata and theories they articulated 
may gradually be reduced to gestalts that facilitate their effective actions with sound understanding of the situations they have faced.

In summary, the participants' gain in new comprehension indicates that the intervention process (particularly POT) has potential to promote academics' learning. The POT experience provided them with a collaborative learning context whereby they watched a colleague teach and shared teaching ideas. Many participants developed collaboration and collegiality through POT which appeared to be supportive of their learning. TT-SET and POT as formative lenses for academics' reflection could bring about their new comprehension about educational topics (e.g., teaching, students, and subject) and reflective abilities. Most academics of Business English in this study reported that the intervention provided meaningful learning for them. Levels of learning varied among academics, depending on which types of reflection they exercised. The new understandings may result in actions which are discussed next.

\subsubsection{Changes to practice and future plans}

For most academics, new comprehensions resulting from reflection triggered action for solving problems of current practice and in planning changes to future practice.

\section{Changes in practice: refining teaching skills or trying new ideas}

Ten participants reported that they refined their teaching or tried new teaching ideas. Bell (2001), in a study on implementation of a teaching development programme, categorised changes into "technical", "pedagogical" and "critical" (p. 33). Participants' perceived changes to their current practice in the present study were also associated with technical changes (e.g., reducing speech flow, timing classroom activities, using audio-visual aids, providing extra materials) and pedagogical changes (e.g., developing students' English language communication skills, collaboration skills in group-work and pair-work, developing students' critical and creative thinking, giving individual support, strategies for motivating students in class). No critical changes were made to current practice.

Changes to teaching were made by ten participants; however, whether academics' teaching is improved or not cannot be concluded. Comparing the end-of-term TT-SET ratings with the early-term ratings, it was found that three academics received higher rating for some items while the others received about the same or lower ratings. This may mean that not every change made was acknowledged by students. Changes made 
by academics may not be noticed by students who would then give about the same ratings on their performance at the end of the course (Penny \& Coe, 2004). Another possible explanation is that students might have noticed the changes but formed an opinion on academics' teaching at the outset of the course and did not want to change their thinking. I did not have data (i.e., no interview data for deeper understanding) of students' perceptions of the changes academics made. Without such data, these results may raise concern about how dependable TT-SET is when used as a measure of improvement. This suggests that some measures should be taken to understand whether students observe and notice the changes academics make; for example, should academics communicate explicit feedback and changes with students and ask for feedback on the changes? However, the main purpose of TT-SET was not to measure teaching improvement but to help academics identify possible teaching problems and areas for improvement.

The data on teacher's reflection reports, video-recordings, and interviews indicate that ten academics attempted changes to improve practice. This finding replicates the outcome that POT could lead to changes (A. Bell \& Mladenovic, 2015; M. Bell, 2001; Donnelly, 2007). Changes made to improve practice could have resulted from a state of dissonance created during academics' reflection process where they may have found a discrepancy between their view of practice and that of students and their peer. To eliminate the dissonance, academics may have changed their behaviours (Brinko, 1993; McLeod, 2014). As presented in chapter 6, TT-SET led academics to set targets for improvement - their aspirations. It concurs with the finding that student feedback promotes reflection which helps academics develop skills (Arthur, 2009). It is also supported by other findings that actively listening to students through formative SETs helped academics improve teaching (Aultman, 2006; Ramsden, 2003; Yao \& Grady, 2005). The study participants set their goals for teaching improvement based on students' feedback on their teaching. Then, their planned changes were guided through POT as a form of consultation. In this study, aspirations together with feedback from students and colleagues stimulated academics to act. This aligns with the literature, which reports that POT helped academics refine teaching skills or try new ideas (A. Bell \& Mladenovic, 2008; M. Bell \& Cooper, 2011; Hammersley-Fletcher \& Orsmond, 2005; Hendry \& Oliver, 2012; Shortland, 2010). Martin and Double (1998) believed that teaching observation and joint reflection with supportive colleagues can 
facilitate the refinement and development of teaching skills. Formative feedback is regarded as a trigger for self-corrective adjustments of behaviours (Bandura, 1977a). Thus, as external sources for reflection, TT-SET and POT provided academics with different perspectives of their practice that may challenge or change their own view of teaching, creating the inconsistency between their beliefs and practice. This then may trigger actions for changes in practice.

Reflection may not lead to enhanced practice if academics do not engage in premise/critical reflection (Hammersley-Fletcher \& Orsmond, 2005; Peel, 2005), do not have substantive knowledge and skills to take effective actions (Jones, 2007; Peel, 2005), and do not have a self-confidence (Peel, 2005). Because academics' knowledge and skills to take actions were not examined, it is impossible to make judgments about whether the academics made improvement (and also, this study was not aimed to measure improvement). Thus, further research on these aspects is needed to measure teaching improvement. However, the finding suggests that the intervention at least made academics become aware of practice and focus on aspects that they identified as needing improvement. Reflecting upon and trying new teaching strategies in the classroom can support academics in propensity towards improvement (Donnelly, 2007). No matter what level of reflection this study's participants engaged in, most of them made changes to their practice.

However, there was a case where changes were not made. An said that she reflected on her practice and mentioned that she would use PowerPoint in future practice. An did not view the problem she described as a real problem because she believed that using 'chalks and blackboard' was as useful as using PowerPoint for lesson presentations. This may explain why she did not make changes (i.e., showing persistent adherence to her approach in current practice). It might be argued that "a problem is unlikely to be acted on if it is not viewed as a problem" (Loughran, 2002, p. 35). However, An thought that the lack of PowerPoint did not actually constitute an instructional problem because what she used worked well. She realised that using PowerPoint may add something better to her practice. This outcome suggests that An began to develop awareness of her practice after the intervention (as discussed earlier). In short, it can be said that the intervention helped most academics make changes to their current practice. However, as theoretical implications suggest, for academics to effectively transform what they learned from the intervention into practice, they may 
need sufficient knowledge, a capacity and skills for reflection, and an ability to take effective action, which may be the areas to be explored in future research.

\section{Planning changes to future practice}

All academics intended to make changes to future practice. Their intention may have been associated with new understandings that stimulated their further aspirations, enhanced confidence, self-efficacy, sense of autonomy, and collegiality (see Section 7.2.3.3 and 7.2.3.4). This is supported by the argument that if academics perceive themselves as competent or self-determining and the changes they make as meaningful or significant, they will sustain the changes (Paulsen \& Feldman, 1995). It should be noted that "By virtue of being personally meaningful, the individual is more likely to be intrinsically motivated to change" (Peel, 2005, p. 501). However, there was not sufficient evidence to demonstrate the enhancement of intrinsic motivation. Nevertheless, sharing understanding and support between several pairs may have enhanced their "zest for further exploration of their practice" (Donnelly, 2007, p. 125). The enhancement of collegiality or a commitment to mutual support, as Cosh (1999) suggested, would be more conducive to academics' willingness to experiment in their practice. Furthermore, POT could promote collaborative conversation about teaching and dissemination of best practice (Atkinson \& Bolt, 2010; Cosh, 1998; HammersleyFletcher \& Orsmond, 2004; Hatzipanagos \& Lygo-Baker, 2006b; Yiend et al., 2014). This finding is supported by several studies which shows that participation in this intervention process at least indicates their willingness and readiness to change (Wlodarsky, 2005), or awareness of it (Bell \& Mladenovic, 2008). The intervention process, particularly POT, made academics more confident, more pedagogically aware, and more willing to experiment in their teaching with intentions for future changes. It is also necessary to note that although one participant (An) did not have a positive experience of the intervention, she became aware of her practice and stated that she intended to take actions for future practice.

Another explanation for intention to make changes to future practice may be that academics may not have had sufficient time to implement changes. For example, Hang claimed that she made some minor changes to current practice and would go on make changes in the future. This is quite understandable because change may be viewed as "a more long-term process of reflection, evaluation and adaptation" (Engin \& Priest, 2014 , p. 7). This means changes may not take place immediately after reflection, and 
academics may need time to evaluate and adapt new techniques or strategies. From these explanations, it can be suggested that most academics appeared to set plans for changes to future practice. TT-SET augmented with POT appeared to have triggered academics' teaching aspirations for personal development.

In summary, in addition to changes to current practice, the intervention also led academics' intention to make changes to future practice. This may have resulted from their reflection and understanding of practice that informed future teaching. Changes for development is an ongoing process of reflection, evaluation, and adaption, and thus need sufficient time to occur. The positive sign from the implementation of TT-SET augmented with POT in this study is that academics reported their intention to make changes in future performance, showing their aspirations for future changes and improvement.

\subsubsection{Enhanced confidence, self-efficacy and autonomy}

The intervention made academics become aware of their teaching ability, capacity to execute actions, and autonomy.

\section{Enhanced confidence and self-efficacy}

Ten participants reported increased confidence and many of them developed a sense of self-efficacy through their new understandings of their teaching and actions to refine practice. Most participants felt confident in their teaching. This finding is supported by many studies that POT may enhance teachers' confidence (Barnard et al., 2011; Bell \& Cooper, 2013; Cairns et al., 2013; Carroll \& O’Loughlin, 2014; Donnelly, 2007; Pressick-Kilborn \& te Riele, 2008). First of all, the intervention, particularly the POT experience, helped confirm teaching methods. For example, Cuc reported that feedback from her students and peer helped her affirm of her teaching methods. Dang learned an effective approach from Khoa to review lessons and became creative. Thus, the academics' confidence developed by the affirmation of their approach based on peer feedback (Bell \& Cooper, 2013) and on the peer's teaching they observed (Engin \& Priest, 2014). Additionally, confidence could be built when academics perceived that they implemented successfully what was suggested by their peer. Binh applied Cuc's advice in minimising dead time and did it successfully. Then, confidence could be built from seeing the positive impact of the changes on student learning. For instance, Dieu knew more about her students and realised they became more interested 
in her teaching. Next, confidence could come from the learning of new approaches. In other words, confidence was also built through reassurance that what was used in their peer's classroom could also be used in their own classroom context (Carroll \& O’Loughlin, 2014; Engin \& Priest, 2014).

Academics' confidence in performing teaching well can be a positive sign of enhanced self-efficacy. As discussed earlier, reflection led to new comprehensions which could trigger actions to change practice. The positive outcomes that academics perceived could be associated with the enhancement of self-efficacy. When a person believes he/she has capabilities to achieve, the person develops a sense of efficacy. There was evidence that academics' sense of self-efficacy was enhanced; possibly POT brought about mastery experience, vicarious experience, and verbal persuasion - possible sources of self-efficacy.

First of all, mastery experience through POT appears to have contributed to the enhancement of self-efficacy. Cuc, Dieu, En, and Nga felt that their practice was better or that they had more credibility in teaching. This attitude of confidence may stem from their belief that they could perform successfully in the classroom. According to Bandura (1977a), people's beliefs about their efficacy can be most influenced by mastery experience. When problems arise, the person believes that he/she can be successful through persistent actions. These academics' perceived success in practice could be related to the enhancement of their self-efficacy, which then could lead to their expectations of successful future practice. For example, Dieu believed she can manipulate her impact on students with well-prepared lessons; En perceived that she applied successfully a new strategy (e.g., teaching vocabulary in context) she learnt from Dieu. This finding replicates the finding that when academics applied new strategies successfully, they developed their beliefs in the usefulness of what they have learned and what they are capable of (Hendry et al., 2013; Hendry \& Oliver, 2012). The intervention appeared to provide the academics with mastery experience which may have enhanced their self-efficacy.

In addition, vicarious experience led to enhanced confidence in academics' capability to manipulate their performance. Vicarious experience or modelling can help develop a belief in academics' ability to use new teaching ideas because they saw these new teaching strategies being successfully used by their peer. A clear example of enhanced self-efficacy is Khoa, who believed that she had the capacity to make effective changes 
new strategies and affect students' learning because she learnt from watching Dang performing them successfully. This finding aligns with other findings that through observational learning (Bandura, 1997), academics could learn from observing their peer's behaviour and vicariously experiencing his/her success (Donnelly, 2007; Hendry et al., 2013; Hendry \& Oliver, 2012). In a study of online POT where teachers observed virtual classrooms and engaged in dialogue with their observed teacher as a 'learning friend', Harper and Nicolson (2013) found that participants perceived an increase in their confidence: "Participants found that observing others' teaching approaches was beneficial in developing confidence in their own capabilities, in reflecting on and challenging existing preconceptions and in widening perspectives" (p. 271). The peer in this study was regarded as a lens for reflection and changes for improvement, so Khoa felt more confident in her own pedagogy when teaching problems were solved and believed she had attained her goal as her peer could.

Furthermore, academics' self-efficacy may be enhanced through verbal persuasion. Khoa also felt confident when receiving Dang's encouragement for her capacity to organise classroom activities logically. This appears to replicate a finding from Hendry and Oliver (2012) that engaging in conversation with the peer may have offered the academic affirming feedback on teaching that helped enhance self-efficacy. It should be noted that there are other cases such as Binh and Hang who felt confident in their teaching skills after receiving their peer's verbal feedback, but it is unclear whether they enhanced self-efficacy. Binh gained affirmation of her teaching when describing similar problems and sharing ideas about solutions with Cuc. Hang, who is in her early career, highly valued the teaching observation and conversation with Nga from whom she got advice on practice and prospects for success. Tschannen-Moran and Hoy (2007) suggested that novice teachers' self-efficacy was likely to be impacted by contextual factors like verbal persuasion. A causal effect between verbal persuasion and Binh's and Hang's self-efficacy cannot be definitely established, but they believed that their peer's suggestion helped them deliver better performance.

In addition, junior academics could learn more when they were paired with an experienced peer. For instance, Hang emulated Nga because she believed that Nga was competent and had higher status as a senior academic. This result concurs with research with younger learners by Horner, Bhattacharyya, and O'Connor (2008) who indicated three characteristics of an effective model: similar, competent, and of higher 
status. Similarly, a participant in a study by Bell and Cooper (2013) found that "observing experienced colleagues teach helped develop a realistic view of teaching standards" (p. 67). The case of Hang provides an example that junior academics can benefit from vicarious experiences through their pairing with an experienced academic. These experiences may contribute to their enhanced confidence in teaching. However, the pairing of junior and experienced academics may highlight a potential problem that the junior may emulate ineffective teaching simply because they respect a more experienced peer. Also, deference will also hinder open critical dialogue (see section 7.3).

In summary, the intervention enhanced most academics' confidence and may have enhanced some academics' self-efficacy. POT could bring about sources of selfefficacy: mastery experience, vicarious experience and verbal persuasion. Participating academics became aware of their capacities in teaching. The finding is meaningful because it implies that to encourage academics to take action it is necessary to promote sources of self-efficacy through POT. The review of literature on teachers' self-efficacy implies that academics' self-efficacy is associated with their willingness to take action to change practice.

\section{A sense of autonomy}

Participants believed that the intervention allowed them autonomy in deciding which aspects of teaching to focus on. It allowed academics to identify and choose the teaching areas for feedback based on their own needs, set their goals (or aspirations), choose appropriate strategies to solve teaching problems through observation of peer's teaching and peer's feedback, and evaluated the outcomes through writing their reflection reports, as featured in Knowles' (1975) description of self-directed learning. Getting desired information may help them gain better understanding of teaching areas that need improving (Rotem \& Glasman, 1979; Stronge \& Tucker, 1999). This finding is useful because academics' autonomy in this sense is similar to Little's (1995) description of autonomy that refers to teachers' capacity to engage in self-directed teaching with personal responsibility through reflection, control, and freedom. TTSET augmented with POT also helped to convey academics' ability to develop appropriate teaching skills, knowledge and attitudes for themselves as teachers, in collaboration with others (Smith \& Erdoğan, 2008; Yan, 2010). The interpretations of teacher autonomy, as Lamb (2008) suggested, emphasise teachers' capability of 
making decisions according to their professional learning needs, or the freedom to do so. Besides, Gosling (2014) mentioned that, among other key features, collaborative peer review "recognises professional autonomy of all parties" (p. 20) and allows peers to discuss issues relating to student learning or their teaching problems in a systematic way. The collaborative POT used in this study had this feature. Autonomy, freedom, and choice are associated with self-directed learning (Brockett, 2006). Thus, the intervention that brought about a sense of autonomy may be viewed as the mechanism to promote self-directed learning.

It should be noted that whether or not a sense of autonomy actually led to actions might depend on academics' attitudes and self-efficacy. There was little direct evidence to make a strong connection between academics' autonomy and action. There is an argument that some academics have the capacity to engage in self-directed activity but are not willing to do so (Yan, 2010). It might be assumed that it depends on whether teachers' self-efficacy was high or low (Skaalvik \& Skaalvik, 2014). Teachers with high self-efficacy may view autonomy as an opportunity to try different teaching ideas. In some cases, the academics (namely Cuc, Dieu, En, Nga, and Khoa) with enhanced confidence and self-efficacy made changes and showed a propensity for personal learning. However, it is not sufficient to make connection between autonomy and action. In contrast, teachers with low self-efficacy may view autonomy as a chance to conceal their self-aware weaknesses and retreat from challenges, which may hinder their personal learning and growth (Skaalvik \& Skaalvik, 2014). Because of the lack of direct evidence, it is not possible to determine whether or not An was making use of autonomy to conceal weaknesses and retreat from challenges due to her low efficacy. However, it should be noted that she did not change practice. She believed in her teaching capability because of many years' experience and was satisfied with current practice.

\section{Summary}

The intervention developed academics' confidence, in some case, enhanced selfefficacy, and autonomy. POT offered participants sources of self-efficacy: mastery experience, vicarious experience, and verbal persuasion. These experiences helped the participants look back on their teaching capacity and felt confident in their ability. Additionally, the steps of the intervention focused on academics' professional needs (i.e., they had freedom to choose teaching aspects for student feedback collection and 
peer observation). Feeling free to focus on their needs brought about a sense of autonomy. There was no substantial evidence to establish actual connection between the enhancement of self-efficacy and autonomy and intrinsic motivation, but most participants showed their aspirations to make change and improve future practice. This indicates a certain level of willingness to take action. The enhancement of self-efficacy and autonomy is useful for future modification of the intervention because these, as fundamental psychological needs, have been believed to be associated with academics' intrinsic motivation (Gagne \& Deci, 2005) and pedagogical practice (Lu, Jiang, Yu, \& Li, 2014; Schwarzer \& Hallum, 2008) such as attempts in teaching, aspirations, enthusiasm or commitment, and persistence to deal with difficulties (Bandura, 1997; Skaalvik \& Skaalvik, 2014).

\subsubsection{Nurtured collaboration and collegiality through POT}

Colleagues' cooperation in the POT process helped develop collaboration and collegiality among some academics. Eight participants reported that POT made peer relationships develop to a new level with enhanced collegiality. Collegiality may include willingness to act as a support person or teaching model, sharing teaching materials, websites or useful resources for teaching. For example, Dang and Khoa perceived a trustful and cooperative atmosphere of their collaboration in the debriefing and cooperative learning from each other. They also joined in further collegial activities such as sharing teaching materials (e.g. sharing video clips, websites or resources they found useful) and wanted to share teaching ideas and be observed by peers. This finding is supported by many studies suggesting that peers develop collegiality, mutual trust or understanding, and friendship when spending much time together (Bell \& Cooper, 2011; Byrne et al., 2010; Donnelly, 2007; Harper \& Nicolson, 2013; Long \& Hoa, 2010; Shortland, 2010). The development of collegiality from the opportunity for peers to collaborate and share ideas and change targeted aspects of teaching appeared significant for the use of SETs in general. Because SETs used in combination with forms of consultation can be effective for teaching improvement (Brinko, 1993; Dresel \& Rindermann, 2011; Lang \& Kersting, 2007; Marsh \& Roche, 1993; Penny \& Coe, 2004; Rindermann et al., 2007), the enhancement of collaboration and collegiality may make POT a promising form of consultation for academics. 
It may be questioned if the enhanced collegiality is contrived collegiality. Hargreaves and Dawe (1990) distinguish between 'collaborative culture' and 'contrived collegiality'. They regarded collaborative culture as comprising of "relationships of openness, trust, and support" in which teachers define and develop their goals as a community whereas contrived collegiality is viewed as consisting of "administratively contrived interactions among teachers where they meet and work to implement the curricula and instructional strategies developed by others" (p. 227). In the process of POT in this study, specific methods were not forcibly imposed upon the academics, and they had freedom to target what to teach, give suggestions to their peer or accept or reject suggestions from their peer. Many academics engaged in open dialogue in a trusting environment. The pairs such as Dang-Khoa, Dieu-En, Phung-Giang trusted each other in POT and they engaged in observing each other' teaching and teaching dialogue after POT. Though Nga in the pair Nga-Hang perceived deference from Hang, the pair then gained understanding of each other. Trust and collaboration are the key for successful POT (Byrne et al., 2010; Gosling, 2002; Schuck et al., 2008; Shortland, 2010); while collaboration and collegiality are regarded as crucial for professional growth in teaching, these elements cannot be imposed but should be let develop freely with the teachers controlling their interactions (Ambler et al., 2014). The academics in this study had freedom to control their interactions with their peer in POT. As evidenced previously, POT appears to have been a learning environment where collaboration and collegiality were nurtured between some pairs of peers.

However, working with and learning from a colleague may raise several issues about: disagreement with feedback and lack of sensitivity, perceived power imbalance and deference (see section 7.3.2), and concern for an unsafe environment for POT. It should be noted that An, Binh, and Cuc did not develop collegiality through POT for several reasons. First, tension developed between An and Binh because of disagreement with feedback and lack of sensitivity which resulted in confrontation. The second reason is the deference Binh paid to Cuc so that she did not give critical feedback that may cause confrontation. The third reason is the feeling of unsafe environment in the department: Cuc said that she could share teaching ideas with a peer but would not wish to be a teaching model for others. She thought her teaching may not be better than others' and was afraid of a possible back-stabbing or nit-picking about her teaching. This fear is not uncommon because academics may find POT 
intrusive, threatening, and subjective (Bell \& Mladenovic, 2008; Thuy, 2012). The feeling of being judged was unhelpful in POT. Avoiding the use of language that infers any judgement and moving towards opening discussion on teaching with peers was a suggestion to solve this problem (Ambler et al., 2014).

In summary, the intervention nurtured collaboration and collegiality between some peers. The development of a good relationship may enhance academics' willingness to participate in and develop their positive attitude towards collaborative learning. In other words, the intervention process created an opportunity for making connections among many academics. This is the positive side of the intervention.

\subsection{Enabling factors and challenges}

The study identified several enabling factors and challenges which depended on academics perceptions of TT-SET, observation of peer teaching, receiving feedback, and time resource.

\subsubsection{Perception of TT-SET}

Academics' willingness to use TT-SET for reflection depends on their perception of its values. Six academics regarded TT-SET as completely reliable and useful for informing teaching, which might have made them willing to use it. This finding seems to concur with Belluigi's (2013) evidence on teachers' perception and use of student feedback, as a lecturer said: "the feedback I solicit through evaluations provides me with insight into how students experience my teaching, our classes, me, and I can then use this to broaden the educational experience for all of us" (p. 12). It appeared that perceiving TT-SET as reliable and valid made academics receptive to TT-SET, which also seems consistent with the result in the Vietnamese context from Dung and Mcinnis (2002) in a study that $57 \%$ of participating academics regarded SETs as valid and $67 \%$ agreed that SETs can help improve teaching. This finding implies that whether academics valued TT-SET as a reliable and valid source for reflection on practice can be associated with their willingness to use it.

However, there was also a challenge that academics would use TT-SET in a limited way or would not use it at all when perceiving it as lacking reliability and validity. Four academics regarded TT-SET as distorted by factors including student carelessness, subjective thoughts, first impression, and lack of responsibility and 
wholeheartedness, and used it an additional source of information on teaching. Students may not have been viewed as mature enough to give reliable feedback, so their feedback was regarded as a blunt tool that needs critique before use. The perception of TT-SET reliability seems to be associated with the perceptions that students are not competent to evaluate academics' performance or skills (Arthur, 2009; Douglas \& Douglas, 2006; Dung \& Mcinnis, 2002; Richardson, 2005). Yet, this perception of students as lacking independent thought, profound perspective, and ability to evaluate their teachers (Dung \& Mcinnis, 2002) might have been impacted by Confucian ideology in which teachers have a special status $(\mathrm{Hu}, 2002)$ in the hierarchical order of student-teacher relationship. This finding is also no surprise when compared to the findings from Stein et al. (2012) and Yao and Grady (2005) that academics who were suspicious of SET did not use it or used it in a limited way.

Academics appear to show reluctance generally to use SETs; another study similarly showed that not every academic "is convinced of the desirability and utility" of SETs (Spooren \& Mortelmans, 2006, p. 201). In another example, conducting a study to determine the effects of the formal evaluation process of two- and four-year college faculty on instructional improvement, Spencer and Flyr (1992) found that 77 percent of the responses indicated that SETs were not taken into consideration when academics made changes to their teaching. In addition, a study conducted at a major Canadian university to explore the utility of student ratings by Beran et al. (2005) showed that although 84 percent of the responses indicated the usefulness of SETs, academics did not usually use it to improve their teaching. However, this is not the case of the participants in the present study because they at least used it to identify teaching problems and form aspirations to change them, although this may well have been because the intervention required them to do so.

There appeared a case (namely An) that the purpose of TT-SET may have been wrongly assumed. An's perception of early-term TT-SET as being unreliable and not accurately evaluating her performance is not consistent with a common view that midterm SET is almost always collected for formative purposes while end-of-term one for summative purposes (Bullock, 2003). An may have misinterpreted its purpose and believed that end-of-term TT-SET would give more accurate evaluation. Her perception might have been shaped by a common sense of SET as an administrative tool (i.e., the summative purpose) in Vietnam where its formative and summative 
purposes have not been clearly defined. The use and purposes of SETs are not well defined in countries where SETs are relatively new (Nair \& Mertova, 2013). This implies that for An to rethink TT-SET, it may be necessary for her to be clear about its formative use.

In summary, the extent to which TT-SET enabled or hindered academics to use it for reflection depends on their perception of its reliability and validity. Many viewed it as useful and reliable. Yet, some were suspicious of its reliability and validity, which might be associated with the impact of Confucianism on their belief about students in the student-teacher relationship.

\subsubsection{Perception of POT}

The extent to which POT could promote academics' reflection and learning depends on their perception of observing the peer teaching and receiving feedback, which appeared to be impacted by the nature of the peer relationship.

\subsubsection{Observing the peer teaching}

\section{Useful opportunity for learning from observation of peer teaching}

Observing the peer teach offered many academics an opportunity to learning from seeing new strategies or referring the peer's problems to their own classroom context. It is easy to find examples that academics (e.g., Binh, Hang, Dang, and Khoa) learnt new strategies because they perceived their peer as using good teaching strategies, having specialised knowledge, and being experienced. Even when academics (e.g., Cuc and Nga) could not learn new teaching ideas, they used the observation as a chance for reflection by identifying problems in their peer's classroom activities and referring to their own situation for solution to similar problems. This finding appears to go beyond the perception that learning that occurs in POT is often attributed to the teacher being observed after he/she receives comments from the observer and engages in reflection (Cosh, 1999; Gosling, 2002; Hammersley-Fletcher \& Orsmond, 2004; Yiend et al., 2014). It replicates the findings from Hendry and Oliver (2012) and Bell and Mladenovic (2008) that academics can learn from observing someone teach as well. Academics can learn, for example, by adopting the peer's practices (Hendry \& Oliver, 2012) or thinking about solution for problem arising in the peer's classroom 
by referring to their situation (Tenenberg, 2016). Whichever way learning came from, observing a peer teach appeared to be useful.

\section{Limited learning from observing the junior peer teaching}

However, the challenge seemed to occur in unequal pairings when senior academics perceived they could not learn much from the junior peer. This means they could not learn better teaching skills from observing the peer because they perceived that their junior peer was not as experienced in teaching. This challenge is characteristic of senior-junior pairs such as Cuc-Binh, An-Binh, Giang-Phung and Nga-Hang. Their peer relationship had a distance deriving from age, experience, and organisational status. This finding indicates a tendency that these senior academics judged their junior peer's teaching based on their assumed superiority of knowledge and experience (Gosling, 2014). It appeared that these senior academics perceived the peer in POT only as a teaching model from whom they could learn effective teaching ideas. Then, it became a challenge because different observers may have very differing ideas about effective teaching, as (Gosling, 2014) found.

\subsubsection{Receiving peer feedback}

\section{Useful opportunity for learning from peer feedback}

Perceiving the appropriateness and relevance of peer feedback might have enhanced academics' willingness to use it. The participants in this study received both written and oral feedback, and the teaching dialogue provided an opportunity for face-to-face exchange. Eight participants valued teaching dialogue with useful, relevant, constructive feedback, as Sullivan et al. (2012) found. The development of collaboration and collegiality (see section 7.2.3.4) appeared to be associated with their perceived trustful and cooperative atmosphere which may be viewed as deriving from the nature of the peer relationship. Productive peer relationships built from trust encouraged reflective dialogue and learning about teaching. There were instances, such as Dieu-En and Dang-Khoa, where the peers trusted each other and engaged in discussion about teaching at the debriefing. This replicates findings that mentoring and support from a trusted colleague are an integral part of its success (Carroll \& O'Loughlin, 2014; Shortland, 2010). The attitudes of welcoming feedback might have enabled these academics to take the opportunity of collaboration with a colleague through POT to reflect on practice and learn about teaching. This kind of open- 
mindedness, a necessary characteristic of a reflective person who is "open to the voices, opinions, and advice of others" (Valli, 1997, p. 68), seemed to be associated with their perceived usefulness of peer feedback.

In another example, a productive peer relationship was built from a non-threatening atmosphere between Binh and Cuc though some challenges were also acknowledged (see the following section). Unlike the collaboration with An (discussed later), comparing An's and Cuc's feedback, Binh felt a non-threatening atmosphere with Cuc and was pleased with Cuc's feedback because of its appropriateness. This may mean Binh did not perceive Cuc's feedback as confrontation or humiliation but as a respectful constructive suggestion. As Schuck et al. (2008) indicated, "respect for one another's expertise in teaching" (p. 224) is an important factor for POT to work. Thus, that Binh's perceived Cuc's respectful attitude in giving negative comments may have helped keep the harmonious peer relationship.

Besides, a productive relationship could also overcome bad feelings when either of the peers gave only discouraging negative comments. For example, Phung overcame the bad feeling (i.e., feeling discouraged) from only negative comments given by Giang who is her peer and close friend because she perceived that Giang was truthful about her feedback. Additionally, Phung agreed with Giang's feedback and saw it as an opportunity for making changes. This is also in agreement with a finding of Carroll and O'Loughlin's study that "some participants viewed such closeness between peers as positive, in that their relationship was strong enough to cope with negative feedback" (Carroll \& O'Loughlin, 2014, p. 449). Arguably, Phung's perceived truthfulness from her friend and receptiveness to negative comments encouraged her in reflection and learning from the peer.

\section{Limited learning from the junior peer's feedback}

However, there were still challenges. First of all, limited learning was associated with not only observation of the junior peer's teaching (as discussed above) but also the junior peer's feedback in those unequal pairings. It was perceived to be associated with junior peer's deference that only positive comments were given. This is true in the case of Binh because she found it hard to give critical negative comments to Cuc. It appears to reflect a cultural aspect that avoiding critiques that may bring about confrontation by showing deference (i.e., showing respect to the senior in terms of age, status or 
position) and saving face (i.e., avoiding humiliation) was a way the Vietnamese academic maintained a harmonious relationship with their peer. In the Vietnamese Confucian collectivist culture, respect is the cornerstone of a relationship (Borton, 2000; Mai et al., 2005; Tuong, 2002). The debriefing session turned out to be backpatting (i.e., offering each other praise or compliments) (Byrne et al., 2010; Cosh, 1999). It can be argued that there is a blurred line between critical-constructive and judgmental feedback. There seems to be a fear that judgments were being made although the peers have closely worked together (Hammersley-Fletcher \& Orsmond, 2005). The feedback process is very sensitive (Phuong \& Vásquez, 2011), and giving critical constructive feedback is a demanding skill that needs to be trained (Cosh, 1998). Perhaps, Binh was not sure of the outcomes of giving negative comments, so she chose a safer way, showing deference. Academics may have difficulty giving negative feedback (Cairns et al., 2013), so they just give compliments to their peer. This case might be an example of power distance in Vietnamese relationships (Hofstede, 1997) that may prevent peers from fully engaging in rich open conversation (Schuck et al., 2008). Another possible argument is that difference in status can foster one-way conversation where one peer can feel disempowered (Gosling, 2014). However, this is not the case in this study because the senior peers expected to receive more feedback from their junior peer. The potential for using POT to enhance reflection is limited if the process fails to facilitate critical feedback (Yiend et al., 2014). In the case of Hang, only positive feedback was not from deference but from her perceptions of Nga's good teaching. Yet, Nga perceived it as deference. These two cases imply that the senior academics' perceived deference appeared to derive from their positionality that they were more experienced and/or had higher organisational rank. Their belief in power imbalance might have challenged their receptiveness to their junior peer's feedback. Thus, the communication between the peers seemed to be distorted by the imbalance of power (Gosling, 2002, 2014).

This unequal pairing appears more beneficial to the junior academics. As found Bell and Cooper's study (2013), the pairing of early-career and experienced academics was supportive of early-career academics. Consequently, participants such as Cuc, Binh, and Nga in the unequal parings felt that more equal pairing would have facilitated more critical feedback. It can be said that the unequal pairing in terms of experience or age is not beneficial to the senior academics' learning. 
However, the senior-junior relationship (a distance deriving from age and experience) between Giang and Phung is different from the above pairs because the academics are close friends. They did not perceive a lack of critical feedback. There may be an argument that closeness can bring about uncritical acceptance of peer's feedback, and peers may not be truthful about each other's weaknesses for fear of hurting each other (Carroll \& O’Loughlin, 2014). However, Schuck et al. (2008) argued that these challenges may exist in any such partnership and can be overcome when the relationship is strong. Both Giang and Phung said that they were truthful with each other and their friendship became closer. Thus, this robustness in the relationship may encourage more critical feedback.

\section{Disagreement with feedback and lack of sensitivity}

The next challenge is the disagreement with feedback and lack of perceived sensitivity in giving and receiving feedback. Three participants experienced negative feelings from peer feedback. While these academics valued the intervention process, it did not work well when they perceived peer feedback as an evaluation or a challenge to their value. The first interpretation was that Binh perceived judgmental feedback, superior attitude, and lack of respect from An - a feeling of being humiliated. Hence, the finding that defensive or bad feelings that arose as a result of the emerging threat eliminated the benefits of POT is supported by the argument that POT needs to be well implemented in a way that creates a safe, non-threatening environment (Fernandez \& Yu, 2007). Perceiving judgments made feedback sessions ineffective (Barnard et al., 2011) and may call POT into disrepute (Hammersley-Fletcher \& Orsmond, 2005). Thus, the challenge appeared to depend on how the peer interpreted feedback. Feedback may be differently interpreted as discouragingly critical instead of constructively critical as intended (Shortland, 2010). Because Binh perceived An's feedback as criticism rather than as constructively intended, tension arose.

The challenge also depended on how the peer gave feedback. In response to An, Binh criticised her practice. The junior-senior relationship seemed to increase the negative impacts of criticism when An disagreed with Binh's feedback (because she did not believe it was true about her practice) and felt irritated. An's reaction may have been associated with the feeling of being criticised without respect. Binh's criticism may have given An a feeling that her values were being challenged. Another explanation for An disagreeing with Binh's feedback and unwelcome attitudes might be based on 
her assumed superiority of knowledge and experience. As Cosh (1998) argued, "In the case of experienced teachers in particular, a natural reaction to explicit or even implied criticism is to become defensive and inimical to suggestions of change" (p.172). In the Vietnamese context, criticism may be viewed as humiliation, which may imply that the person who gives the critiques does not show respect to the other. In Vietnam, peers have to show respect for each other's age and experience (Borton, 2000; Thang, 2013), and this may be a sensitive aspect to be considered in post-POT dialogue; otherwise, discussion will fail (Fullerton, 2003). This perceived lack of respect from the peer created tension between them, resulting in a negative feeling and defensiveness. One might also argue that An's reaction can be associated with An's special characteristic that she rejected everyone's feedback and showed explicit disagreement because Binh is a junior. It is close to the argument that academics tend to view critical feedback in a negative way (Hammersley-Fletcher \& Orsmond, 2005). This possibility may need to be further studied.

A lack of sensitivity can be born of lack of balance of negative and positive feedback. Giang's only negative comments appeared discouraging to Phung. This finding replicates findings from MacKinnon (2001) that giving just negative comments was discouraging and produced a negative feeling (e.g., disheartened, frustrated) by the lack of recognition of the peer's strengths. However, that Phung accepted it as a chance to improve teaching and the close relationship where peers trusted each other may have helped her overcome this negative feeling (as discussed above).

It should be further noted that the problems (e.g., senior peer's disregard of junior peer's feedback, deference, or saving face) arising in pairs of experienced and inexperienced academics might be examples of a universal issue: lack of parity and reciprocity. This study did not have data for comparing how academics from different cultures react to such issues. However, the interpretations of the findings were based on studies on the Vietnamese culture with theoretical assumptions, what several participants believed, and the perspective of the researcher (i.e., as a member of the Vietnamese culture who has insight into that culture). For example, Cuc believed that Binh might have paid deference to her, and Binh also admitted that she had deference for senior peers. Besides, the lack of sensitivity in giving feedback may have caused a loss of face. I believe that it seems especially damaging when a Vietnamese teacher loses face. In a collectivist Confucian culture such as Vietnam, people believe that 
losing their face also means losing face of their family, group, or even organisation. There may be elements that are different from more individualistic cultures, and further research is needed to examine if such elements are particularly associated with the Vietnamese culture (i.e., to strongly justify if such elements have distinctive implications for Vietnamese academics).

In summary, academics' judgmental attitude, perceived lack of respect, delivery of imbalanced positive-negative comments detracted from the benefits of POT. Criticism of one's teaching may cause a negative feeling in all cultures, but it may be more damaging in the Vietnamese culture. The cases indicate how academics can give and receive feedback and take care of the nature of the peer relationship in teaching dialogue with regard to mutual respect. Academics may need to know how to give critical constructive feedback without challenging each other's values or causing a confrontation, because untrained academics can cause negative feelings and defensiveness (Cosh, 1998). Also, giving feedback may need to balance negative comments and recognitions of peer's teaching to avoid being disheartened. The finding in this study suggests the importance of maintaining mutual respect in giving feedback is important for POT.

\subsubsection{Time resource for POT}

Limited time was one of the big challenges for academics to fully engage in POT. Although nine academics thought that a shortage of time would be a challenge, four (Binh, Giang, Hang, Nga) actually found it hard to engage in POT because of their workload and limited time available to observe each other's teaching and to make changes. All participants in this study had a full schedule because they often had different teaching sessions in different institutions. This finding is similar to Huong's (2014) finding in that in the Vietnamese context academics had less time to engage in teaching and research due to heavy workload and working part time. There are more part-time academics, and full-time academics have more working hours with various responsibilities (Eddy \& Garza Mitchell, 2012). The participants in the present study were in a similar situation, so spending much time working part time reduced their engagement in POT. The researcher's journal shows that it was difficult to arrange pre- and post-observation meetings that suited both peers, as Long and Hoa (2010) found. This finding also concurs with studies on peer review of teaching involving 
POT in Australia such as Harris et al.'s (2008) and Barnard et al.'s (2011) studies in that academics had to compete for time in their workplace. Time management for POT may be a problem concerning participant availability (Harper \& Nicolson, 2013), preparation for POT (Donnelly, 2007), or too much time and paperwork required for POT (Adshead et al., 2006; Donnelly, 2007). Therefore, the time for POT added to their heavy workloads (Sullivan et al., 2012; Trujillo et al., 2008).

In addition to the problems with time management and preparation for POT, duration of the POT programme was viewed as insufficient for changes. These four academics thought that one semester was too short for them to make changes and suggested that POTs should be scheduled for two semesters. This may imply that professional learning through POT is a continuing process. As Engin and Priest (2014) stressed, POT is not a one-off activity and may not bring about immediate changes because development is an ongoing process of observing, reflecting, and learning. Because of the time constraints, academics may continue their previous practice or adopt ineffective practice. Although reflection may bring about knowledge of good practice, in a stressful environment people may not act in accordance with what they believe is good practice (Forde et al., 2006). Therefore, for effective changes, it is necessary to engage academics in a cycle of observing, reflecting, and learning. In short, limited time challenged academics' engagement in POT, which could have reduced the quality of their reflection and learning. 


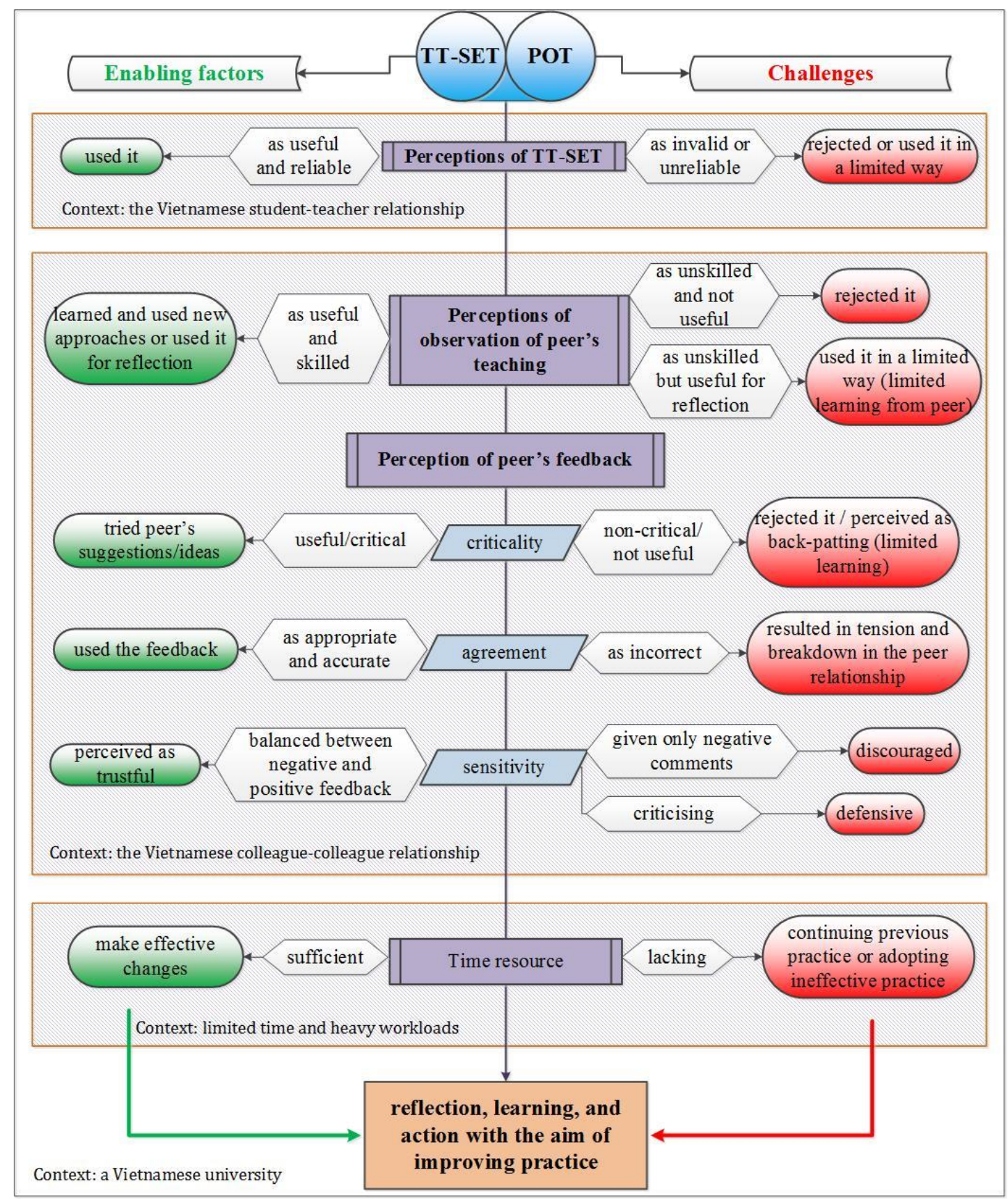

Figure 7.1: Perceived enabling factors and challenges to the intervention

In summary, there were several enabling factors and challenges (see Figure 7.1) that impacted academics' reflection and learning through student and peer lenses. Academics' use of TT-SET depends on their perception of its usefulness, and their limited use or rejection of TT-SET is associated with their view of its reliability and validity. This view seemed to be impacted by academics' view of students in the hierarchal order of the student-teacher relationship. Similarly, the academics' awareness of their position in relation to peers, regarding social connections, academic rank, and experience, affected their interpretation of POT, including perceived 
usefulness of observation of peer's teaching and feedback. A trustful, non-threatening and cooperative atmosphere are important for the collaboration between the peers because it facilitates the peer collaboration. This kind of atmosphere depends on the nature of the peer relationship and the manner in which they communicated with each other. The associated challenges include senior limited learning from the junior peer lens (due to lack of experience and non-critical feedback from deference) and disagreement with feedback and lack of sensitivity (due to attitude of superiority, criticism in giving feedback, and giving only negative comments). These findings suggest that the peer relationship is the critical underlying factor in the collaboration between peers in POT. Another challenge was time constraints and heavy workload that reduced academics' engagement in POT and making changes.

\subsection{The usefulness of the theoretical framework}

The intervention process appeared to fit into a model for promoting academics' reflection and developing their understandings of practice in the Vietnamese context. It is a structured process that provides formative feedback information on practice supportive of teachers' learning. However, several challenges need to be fully addressed for a successful intervention. A revised framework may be necessary for further research.

TT-SET augmented with POT appeared to be a structured or systematic procedure for academics' reflection. The student and colleagues lenses fulfil the role of 'structural aids' or 'external impetus' (LaBoskey, 1993) to reflection. These two of four lenses that Brookfield (1995) suggested engaged academics in examining their practice from a variety of viewpoints (Forde et al., 2006; Loughran, 2002). External perspectives play a critical role in enhancing self-awareness and transforming understanding (Peel, 2005). These lenses were structured to be a systematic approach to practice that supports reflection. TT-SET may complement POT appropriately in this structured intervention which was beneficial to academics' reflection. As students are direct recipients of the teaching process (Kelchtermans, 2009), they could provide a source of feedback that may have encouraged academics to identify their teaching problems and set aspirations for changes. Meanwhile, POT provided them with observation experience and professional conversations with a colleague who can provide a professional perspective on practice (Hutchings, 1996). Student and colleague lenses 
are external resources that are supportive of reflection and can help academics gain insight into their practice.

The theoretical framework developed for this study also explained Shulman's idea of teaching that emphasises comprehension and reasoning, transformation and reflection. It is relevant for examining academics' reflection and reflective practice, knowledge development, and actions. TT-SET augmented with POT facilitated participants' articulation of teaching beliefs. For example, teaching observation and the follow-up debriefing offered an opportunity where peers could share teaching beliefs and theories, teaching techniques and strategies, teaching experiences, and teaching contexts. These discussions between peers related to their comprehension of subject content knowledge and use of the relevant pedagogy to impart teaching. Thus, reflection from formative information obtained from students and peer helped academics develop new comprehension about their practice. This is consistent with Shulman's (1987) ideas about the development of pedagogical content knowledge in his model of pedagogical reasoning and action. In other words, it focusses on processes involved in teaching including the transformation of knowledge into teachable forms: "during this cycle of processes, pedagogical content knowledge is used and generated" (Webb, 2002, p. 241). Through the process of collecting TT-SET for teaching aspirations, observing a peer teaching, receiving peer written and verbal feedback, writing reflection reports, academics became aware of their practice and generated pedagogical content knowledge. This awareness can be viewed as one that "creates opportunities for professional growth and development" (Osterman \& Kottkamp, 1993, p. 19). It facilitated learning and triggered actions for changes to practice. The benefits of POT can be summarised, using Marshall's words: "the power of peer observation resides in its developmental and collegial orientation and its exposure of colleagues to affirmation, constructive criticism, and the experience of how others teach differently" (Marshall, 2004, p. 187).

However, there are several challenges to be addressed. These challenges are associated with academics' perception of TT-SET and POT: TT-SET reliability and validity, observation of peer teaching, and peer feedback, and time for POT (see Figure 7.1). It seemed that in some cases academics' perception was influenced by Vietnamese cultural values which appeared to be a filter for interpreting POT experience. For example, several thought that students could not have independent thought and their 
feedback was distorted. In other cases, academics positioned themselves in the peer relationship based on age, experience, or organisational rank. Thus, positionality affected the way they interpreted the POT experience. For example, in unequal pairings, some senior peers had limited learning from junior peers because deference hindered open teaching dialogue. In one case, unequal pairing brought about one's feeling superior over the other. Then, disagreement with feedback and lack of respect in giving feedback resulted in a relationship breakdown. These findings suggest that the nature of the peer relationship appears to be the contextual factor underlying how academics interpret the POT experience. In addition, limited time influenced academics' engagement in the intervention and action for changes. The identification of these challenges are important for revising the theoretical framework which may be used for further research. Hence, the application of TT-SET augmented with POT may require an addition of the enabling factors and challenges to the model for it to be successfully implemented in the Vietnamese context (see Figure 7.2).

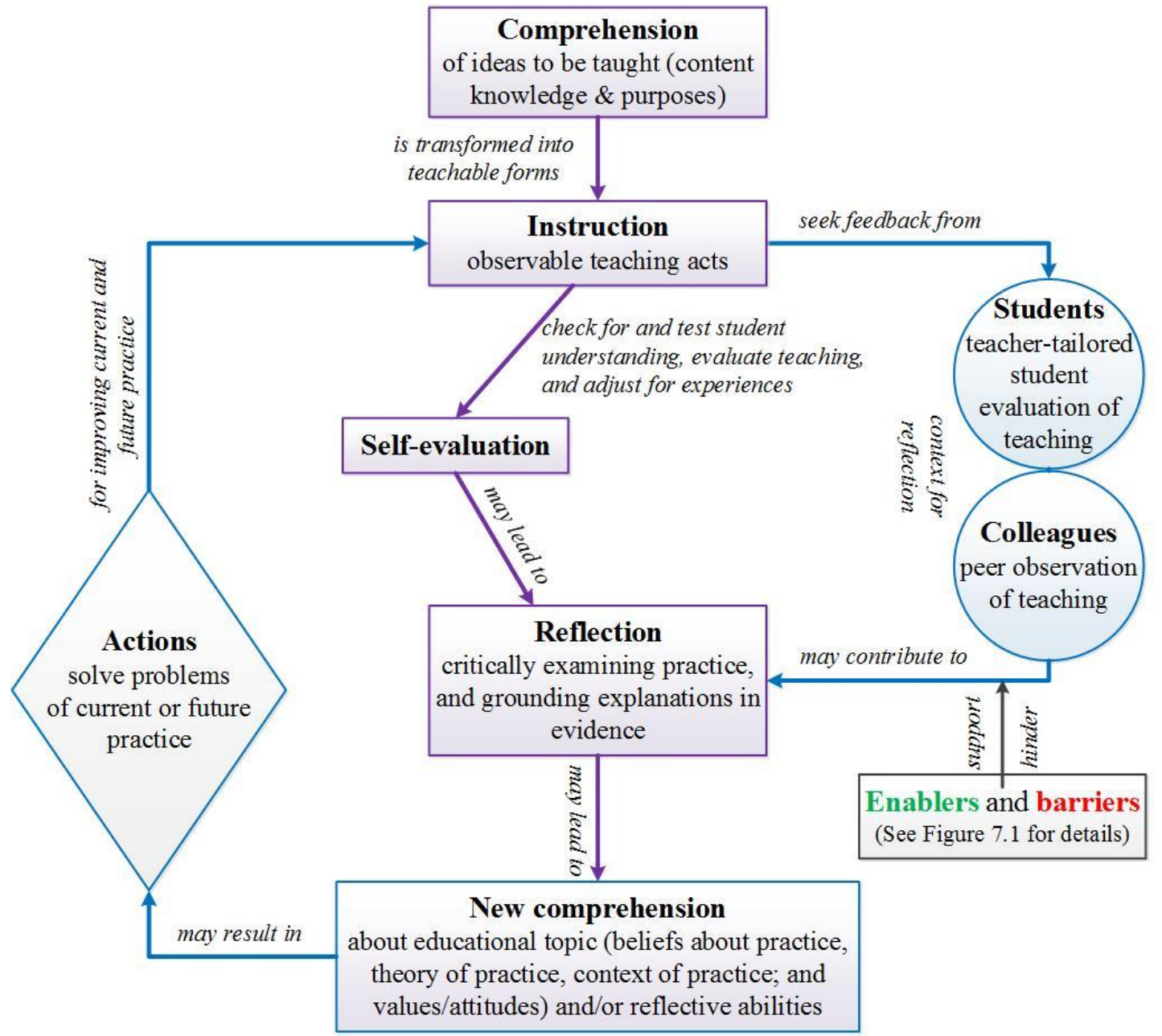

Figure 7.2: Proposed model for the implementation of TT-SET and POT 
In summary, the intervention provided academics with a context for reflection and learning. Particularly, POT was applied in a new context, which brought about understanding of how it worked in the Vietnamese context. These insights are significant for the modification of the intervention if it is to be implemented and further research into this area. 


\section{Chapter 8. Concluding Remarks}

This chapter presents the study's significance and limitations, discusses implications, raises recommendation for future research, and ends with concluding remarks.

\subsection{Significance}

This study is theoretically and practically significant. Particularly, the investigation of TT-SET augmented with POT, in a university in the Vietnamese context contributes to the understanding of this approach in depth. This case examination helped explore whether the outcomes found in other contexts can be expected to this new context and what new issues emerged.

From a theoretical aspect, this study contributes to a critique of a mechanism for academics' changes in pedagogical reasoning, particularly reflection. The theoretical framework developed from the review of literature was relevant to inform the study but is also a contribution to the research field. It provides an understanding of how reflection can be influenced by external lenses that contribute changes in academic staffs' learning and actions to solve practical problems. In other words, the intervention that provides academic staff with varied lenses for viewing their practice (by addressing the what, how, and why of teaching) appears to be a mechanism for their changes in pedagogical reasoning and actions. Testing the use of the lenses in the Vietnamese context provides an understanding of the applicability of a commonly viewed as Western-developed process in a different local context.

From a practical aspect, the study, which is exploratory in nature, helps clarify what is necessary for effective reflective academics and what hinders their reflection and professional learning. The discussion of enabling factors and challenges to academics' reflection from TT-SET and POT helps identify necessary and sufficient conditions for the intervention to be effective. Unlike the university's standard SET and random supervisory observations of teaching that seem not to affect academics' action to improve teaching, the intervention was a structured process that promoted academics' reflection and insight into their practice. Previously, a few studies in Vietnam have been conducted on POT for beginner teachers but have not identified the conditions for POT to be effective, particularly for more experienced academics. This study identified several important factors. Academics' perceptions of TT-SET and POT 
influence the extent to which they used them for reflection. For example, several participants viewed TT-SET as distorted and used it in a limited way. Therefore, if TTSET reliability is perceived, academics may use it. The positionality of Vietnamese academics through POT contributes to conceptualisation of a peer with respect to the characteristics such as age and experience or expertise and the role of the peer. Issues, for example, concerning peers' skills of teaching and criticality of feedback, seemed to occur with the unequal pairings which appeared conducive to junior peer's learning. Several academics (particularly senior academics) believed that more equal pairings would be more productive to their learning. This suggests that more equal pairing would encourage more critical feedback and learning. Another issue associated with the nature of the peer relationship is the perceived atmosphere between peers which was influenced by the distance and closeness in the peer relationship (e.g., junior peer's deference in giving feedback). A trustful and cooperative atmosphere can promote collaboration, and collaborative reflection requires strengthened collaboration and collegiality between academics through POT. The way of giving feedback can cause disagreement and confrontation (e.g., causing loss of face) or bad feelings. For example, lack of respect for peer's teaching can damage a relationship; giving only negative comments can cause bad feelings. These examples suggest that training in feedback skills and interpersonal communication (e.g., how to give constructive feedback without causing humiliation) is necessary for peers' collaboration in POT. In the Vietnamese context, the difference in status deriving from age, experience, and organisational rank sometimes matters. Thus, these ideas replicate Gosling's (2014) two principles for collaborative POT: parity and reciprocity. Furthermore, limited time due to heavy workloads is an issue to be addressed if the intervention is to be implemented. Addressing this issue may require the engagement of middle and top management.

In summary, this study provides theoretical and practical implications on the design and implementation of TT-SET augmented with POT. It discusses educational messages related to academics' pedagogical reasoning, particularly reflection in the teaching process. The outcomes of the study can inform what modification should be done to the framework if the intervention is to be further implemented in the university, with reference to the enabling factors and challenges. Academics' perceptions of the effectiveness of TT-SET and POT provide valuable information or guidelines for 
institutional policy makers and middle and top management if the intervention is to be considered for promoting teaching improvement at the university.

\subsection{Limitations of the study}

There were several limitations of the study. First, the time allotted for this study's data collection was one semester which may be too short to document a comprehensive picture of academics' reflection and changes. The changes in management and heavy workload could have affected the academics' wholeheartedness in POT. The academics' tight schedule also seemed to affect their engagement in the project. Limited time was spent for thoughtful feedback and solutions to pedagogical problems. Thus, this could have resulted in inadequate feedback and deep thinking in writing reflection reports. Some pairs of peers could not arrange a post-observation meeting right after the observation session, so their deep reflection on important aspects of teaching could be limited.

Second, the early-term formative TT-SETs were collected near a long holiday break, so students may not have been concentrating on providing the academics with high quality feedback. This could affect the academics' reflection on practice. In addition, this short period of a semester was not adequate for students to observe changes made by academics, which could raise a concern about the dependability of TT-SET being used as a measure of changes.

Third, student learning outcomes were not documented, so it is not possible to judge whether the intervention could have a significant impact on student learning. This study did not intend to measure students' achievement. However, documenting student learning outcomes may add to the comprehensive picture of teachers' learning through the intervention. This may be an area for future research.

Finally, the study did not examine the study context in details, so it cannot be determined how every contextual factor impacts on TT-SET augmented with POT. More research needs to be conducted to identify the features of a facilitative environment for TT-SET and POT.

This study was conducted with expectations that how the intervention would work in the Vietnamese context on the basis of the review of the literature (e.g., for developing the theoretical framework) and the researcher's personal experience. I believe that the findings confirm my optimism that the intervention could be potentially a valuable 
way of supporting academics' professional development. Hard work was attempted on the analysis in order not to allow the influence of biases on interpretations. It should be noted however that the findings depend on the interpretations of a single researcher and therefore might be affected by his biases. Although the researcher's position was made explicit (see section 3.5) to address issues of reflexivity, it is possible that these interpretations still reflect to some degree his beliefs and experiences.

\subsection{Implications}

The findings of this research offer implications about the application of TT-SET augmented with POT and consideration for institutional culture and policies.

\subsubsection{Implications for practice}

The analysis of the positive outcomes and challenges to the intervention provides direction for improving the practical design of the intervention.

First of all, based on the findings that many academics engaged in content and process reflection only, to enhance learning it would be helpful to promote academics' premise reflection because it can lead to deeper or transformative learning. Thus, a conceptual framework or design facilitating the implementation of TT-SET and POT should be provided and guided so that it can trigger premise reflection. When academics have a road map of what they are going to do and how they will do it, they can establish effective agendas for their personal growth. POT protocols could be revised so that academics can explore 'why' they are teaching. For example, the peer observer's feedback form and the debriefing could contain questions (e.g., why do you think that approach will work well? On what basis did you choose the strategy?) that may facilitate open debate on the choice of teaching ideas.

It is necessary to make explicit the principle of mutual respect for peers in addition to the principles of parity and reciprocity in order to help academics gain a deeper view of what they can learn from observing someone else's teaching, and introduce to them other academics' positive experience of POT. The findings on senior academics' limited learning from the junior peers indicate that senior academics often positioned themselves higher and viewed their peers as lacking experience or skilled teaching and giving non-critical feedback. Senior academics in some cases made judgments on their peers' teaching based on assumed superiority of knowledge and experience. Their 
assumption may be correct; however, it is suggested that in peer-supported review the peer is framed not from the position of a knower who provides solutions to teaching problems but as one who cooperates, gives opinion, and collaboratively explores new teaching ideas (Barnard et al., 2011; Gosling, 2014). The principles of parity and reciprocity need to be made explicit to peers in their teaching dialogue such as the communication between peers being open to challenge from either side and both peers being equally open to learning (Gosling, 2014). However, this study's findings also indicate that some academics found a lack of sensitivity associated with criticism or judgmental attitude, lack of respect (e.g., attitude of superiority), and only negative comments. Thus, a principle of mutual respect may be established such as balance of negative feedback and recognition (MacKinnon, 2001) and showing respect for each other's teaching (Schuck et al., 2008) to avoid causing bad feelings or possible confrontation. In addition, when academics reflect deeply what they can learn from their peer, they will be willing to collaborate. Their insight into the usefulness of POT can also come from communicating positive outcomes from other academics participating in POT. Therefore, if academics have freedom to choose their peer for POT and believe that the peer can be supportive of their learning, they will act accordingly to correspond with the principle of mutual respect, parity, and reciprocity for a productive peer relationship.

Training academics to collaborate in POT is necessary and should take into account the cultural aspects within the sub-culture, the organisational culture, or the society. For academics to engage in effective open teaching dialogues, they need to be trained and equipped with necessary skills (Cosh, 1998) to work collaboratively, give and receive feedback, and avoid confrontation. For example, to deal with direct comments that may sound like criticism and cause humiliation, training sessions may introduce a teacher's simulated teaching and ways of giving feedback using eliciting techniques (e.g., peers eliciting ideas from each other by using thought-provoking questions and giving observational evidence such as students' reaction, teaching style, etc. which helps academics reflect on their practice). Thus, how the cultural issues are addressed during this training depends on the nature of the culture where the academics work.

Academics in this study paid attention to the characteristics of the peer. Many liked to have a peer who has similar experience and with whom they have a close relationship, which they believed would encourage open teaching dialogue. In case of experienced 
academics, they wished to have a peer who was as experienced as they were. However, these academics and a few others also liked to have an outside expert observer. As Gosling (2014) argued, sometimes an outsider is more effective by asking more challenging questions. The data showed that academics thought about their status (as regards age, experience, expertise, and organisational status) in accordance with their peer's position, which is the essential factor that contributes to their interpretation of POT experience. Hence, in order to attain academics' willingness for collaboration and enhance the quality of collaboration, it is necessary to allow academics to explore what peer dynamics work for them. Thus, there is a possibility of blending the peer review model (i.e., the collaborative POT where academics observe each other) and the development model (where an expert observes an academic). Academics may have their own choice on whether they may wish to collaborate with a colleague only or a colleague and an expert. Academics' collaboration and collegiality can be promoted through making explicit academics' view about learning from a peer of their choice. When they choose a peer and have a clear reason or idea of what they can learn from the peer, they may collaborate well with their peer. Harmony in the peer relationship through a peer of choice can help build trust between peers.

The present institution does not have a system for TT-SET and POT to support and promote academics' reflection and learning to teach. When academics have insights into how the combination of TT-SET and POT can contribute to reflection and learning, they may use it to promote their pedagogical reasoning. If the intervention is to be implemented, the purpose and use of TT-SET and POT should be clearly defined. Guidelines on how to carry out this process should be included in a published document which will be given to academics as a supportive resource. Guidelines such as how to develop TT-SET, POT protocols (mentioned above), how to give feedback, definition of POT, etc. should be included in this document. The compilation of a peer partnership tool-kit as resources for POT would be valuable, as the one in Barnard's and colleagues' (2011) peer partnership programme.

\subsubsection{Implications for institutional culture and policies}

Although academics are under time pressures and it is hard to require them take on more burden, the whole university and the academics themselves have a responsibility to improve the quality of tertiary teaching in accordance with the Vietnamese 
Government's (2005) 2006-2020 project to improve tertiary teaching quality. However, how to encourage academics' commitment to ongoing improvement of teaching is often a problem. Although the government has established strong agendas to improve tertiary teaching quality, and the university also has strategic plan for this objective (which sometimes academics contribute to), academics often appear to be less concerned about it. In order to support this goal, it is necessary to motivate them to improve practice.

\subsubsection{When the benefits outweigh the cost}

If the intervention is to attract academics' participation, it is important to help academics see that the benefits they receive outweigh the costs they spend. Although most participants regarded the intervention as useful for their reflection and learning, many realised that time was a challenge for their engagement in POT and changes. Gosling (2014) suggests that to avoid academics' rejection of POT as a waste of time, "the outcomes need to be valued by both individuals and by the institution" (p. 27). To achieve such values for individuals, Gosling suggests a number of ways, several of which support the mechanism of the intervention and the findings of the study, for example:

- allow participants to choose the topic for review. This suggestion is consistent with the way the intervention in this study worked. Participants chose aspects of teaching to be observed.

- allow participants to choose their peer. However, they may choose an expert for teaching observation. This means they may choose whether they participate in the development model or collaborative peer review model.

- provide opportunities for the outcomes of collaborative POT processes to make a difference to approaches to teaching and student learning-not just for the individual but also for others working within the same community of practice. This means the outcomes of the peer collaboration should be shared within learning group or community through activities such as presentations in seminars. When academics realise what they do is significant and valued, they may continue to do it.

- ensure that expectations about the time to be spent on POT are realistic and not over-burdensome. This suggestion is particularly relevant for the current 
situation of academics' heavy workloads. To encourage their participation, appropriate scheduling for POT is necessary. This can be achieved in practice if the university recognises the potential for POT. They would set a timeframe for POT (i.e., as one of academics' responsibilities that is encouraged) as they do for academics' research.

- reduce reporting requirements to a minimum. This point is included because many academics in this study struggled with writing reports. I believe that choosing time-saving means of reporting is critical. For example, academics could audio-record their discussion with peer and their reflection.

Another way to encourage academics to spend their time in POT could be by offering incentives. Six academics thought it would be reasonable to encourage participation that way. Because many academics often have teaching sessions at other universities, they may think twice about the financial loss when engaged in POT. External motivators could be created to encourage academics' effort for professional development. Personal encouragement, rewards and incentives would show academics that their institution recognises and values their participation (Lomas \& Nicholls, 2005; Potter et al., 2011). It suggests that activities to present the outcomes of POT mentioned above can be potentially used as records for payment, bonus salary, or even teaching excellence awards. If this outcome is valued by the university and the academics are recognised for their effort to participate in the intervention, the academics may consider spending their time on it.

Furthermore, academics could have freedom and flexibility to establish their agendas and design steps in the intervention to suit their schedule. This also means giving academics ownership of the intervention process (Potter et al., 2011). Such flexible design can allow academics to arrange time for participation.

In summary, in a context where academics have limited time for the intervention, it is still possible to encourage them to participate in the intervention provided that recognition (e.g., through giving bonus salary or awards), positive outcomes, and flexible design of TT-SET augmented with POT are addressed and communicated clearly with the academics. 


\subsubsection{Building colleagues' relationships}

Through POT, it is evidenced that some pairs developed collaboration and collegiality while others did not. The reasons for failure of collaboration and collegial relationship include deference from junior peers, senior academics' attitude of superiority (when there was difference in status deriving from age and/or organisational rank) and fear of being judged. To facilitate collaborative learning through POT, it is necessary to establish a supportive and constructive collegial environment and a culture that values this kind of scholarship of teaching (Barnard et al., 2011). The nature of the peer relationship appeared to be important to POT success. Peers need to have strong collaboration and collegiality to work out systematic critical reflection and initiatives for teaching improvement. Gosling (2014) discussed the two principles of collaborative POT: parity and reciprocity. According to Gosling, parity is an ideal condition where peers' communication is not distorted by power imbalance and peers "are committed to rationality, openness, equality and to finding truth" (p. 22). Because positionality - the way in which academics see their status in relationship to their peer's status based on age, experience, or organisational rank - is a factor affecting the academics' interpretation of POT experience, equal pairing would solve this problems. In Pressick-Kilborn's and te Riele's (2008) study, with a belief that POT would be more beneficial between peers of equal status, they found that their peer collaboration, built on respect and trust, provided them with support and motivation despite some instances of disagreement. Caring about reciprocity is creating spaces where collaborative peers accept POT for mutual or reciprocal learning and "are equally open to professional learning" (Gosling, 2014, p. 23). Here the role of middle management is crucial. Gosling (2014) gave examples of studies that heads of department, for example, are often critical in influencing the academics' attitude to POT. As Barnard et al. (2015) discussed, developing positive working relationships is essential for cultural organisational changes, and the role of distributive leadership where a clear vision is important because it seeks to empower relationships and share responsibility. Potter et al. (2011) suggested: "Creating a culture in which faculty can learn about teaching from each other requires trust, appropriate rewards, and incentives, along with the careful design of opportunities" (p. 31). Establishing such a culture of learning requires support and engagement from institutional leaders, particularly middle management, to coordinate academics. When academics establish strong interpersonal 
relationship, they can join in dyads or triads to carry out reciprocal POT regularly over a longer period of time such as two semesters.

\subsubsection{Building a group for collective reflection}

Two participants suggested that a group of academics who have similar interest should be established to discuss aspects of teaching and learn from each other. Although it is unknown whether this form of network should be formal or informal, forming such a group is appropriate for maximising the benefits of the intervention and address several challenges. Building a network among these academics may engage them in ongoing reflection, as Barnard et al. (2015) found that "critical ongoing reflection is facilitated by professional learning networks" (p. 37). Thus, the role of leaders in promoting connection among colleagues is important when they establish a routine of collaborative learning. For example, building a learning group through POT and introducing to others how it contributes to academics' learning may stimulate their willingness to participate when they recognise its outcomes and values. In addition, if there is a group for academics to share teaching ideas, collective reflection would be promoted, because reflection is basically a collective endeavour though it can be a private activity (Brookfield, 1995). Collective reflection is crucial in gaining insights into one's own teaching, as Boud et al. (2006) suggest in discussing the notion of productive reflection. Thus, besides their peer, academics can validate their teaching approach in discussion with other members in the group. One may question if it is possible to build such a group of critical friends in a culture that cannot bear loss of face. Although challenges relate to this issue occurred to the pair An-Binh (e.g., perceived superiority and criticism) or Cuc-Binh (e.g., deference), it does not mean such group cannot be set up because the other pairs could collaborate and developed collegiality. When the principles of parity, reciprocity, and mutual respect are communicated clearly, academics can benefit from collaborative learning. For a learning group to be productive, it also requires a leader, particularly middle management, to take serious consideration of structural and cultural factors (e.g., hierarchy, saving face, or collegiality) within the environment whereby academics work. They may address these factors in ways such as training, establishing a routine, or making it a policy so that academics can gain insight into the intervention process and are willing to participate. 


\subsection{Recommendations for further research}

Based on the results and the identified limitations of this study, this section presents recommendation for further research. First of all, the impact of unequal pairing and equal pairing on POT are worth exploring. Research is needed to understand the extent to which power imbalance deriving from age, experience, and organisational rank restrict the effectiveness of collaborative POT. In addition, it is unknown if the difference in age, experience, and organisational rank influences collegiality between peers. Thus, further research is needed to look into this issue.

It would require a much longer study which might also have other benefits. The academics in this study participated on two occasions of POT during a semester. Some of them said that a semester was not enough for them to make changes. Therefore, further research needs to focus on whether a longer time (e.g., two semesters or more) for POT could promote better practice (e.g., significant changes in reflection and teaching performance). The frequency of POT in the period also needs to be examined in order to explore if it affects academic's changes.

A longer study would also enable an examination of the effect on student learning outcomes. Students' learning outcomes and their perception of academics' changes were not included in this study. Because students are the beneficiaries of the academics' teaching process, their learning outcomes and perception should be included in measuring the effectiveness of academics' changes through the intervention process.

The design of TT-SET and POT in this study did not embed cultural issues. One of the challenges is related to disagreement with feedback and lack of sensitivity in manner of giving feedback, which caused confrontation and a relationship breakdown. Thus, cultural aspects for academics to address in communication with peers are worth exploring. Necessary skills to give negative comments, deal with disagreement, and avoid confrontation need to be examined.

Another area for future research may focus on how academics can identify real teaching problems from TT-SET. The case of Khoa (see section 6.1.1) suggests that students may misunderstand what needs improvement and not perceive what they actually need for learning. Therefore, it is necessary to explore ways to help academics know what students really need for learning and identify areas where improvement is needed. 
This study focuses mainly on academics' reflection on practice. Thus, it would be useful to examine whether TT-SET or POT impacts on reflection in practice. Examining the judgements and decisions academics make while they are teaching may be important for establishing a mechanism for academics' ongoing and deeper reflection. Reflection in practice may be a way for academics to judge whether the teaching strategies suggested by their peer are actually an appropriate way of solving the problems they have identified.

\subsection{Concluding remarks}

The study results have practical and theoretical significance. They offer an understanding of what works, how the intervention works, and why it works in the Vietnamese context. The findings also contribute to the discourse of academics' reflection and reflective practice as well as the use of TT-SET and POT for reflection. The study also generates implications for practice and institutional culture and policies. There was a lack of a culture of questioning teaching practice as well as a culture of collaborative learning at the study site. Therefore, it is necessary to establish a culture of reflection and reflective practice so that academics can engage in their ongoing improvement of teaching. TT-SET and POT provide two important lenses for academics' reflection. POT as a form of collaboration can be used for collaborative learning and exchanges among academics. The study may also raise concern about the impact of the Vietnamese Confucian collectivist cultural values (e.g., harmony, facesaving, and hierarchical order) on academics' perception of the peer or students. These values affect the interpersonal relationships between academics in unequal pairings and the perceived relationship between academics and students. Time is also a critical indicator for academics' participation in POT. The intervention can work in such context provided that the challenges are addressed. Addressing these challenges may require the engagement of middle and top management. The analysis offered implications on the practical design of the intervention, the establishment of institutional culture and policies.

Research into how evidence-based approaches such as TT-SET augmented with POT could facilitate academics' changes and action in order to improve teaching is an ongoing process. The nature of reflection and change in practice is in itself a continuing process. Academics may need time to collect information on their teaching, 
reflect on it, and make changes to it (and may refine more often). In other words, academics' learning and refinement of practice is likely to be recursive. Then, more studies need to be conducted to examine other aspects of the intervention. This means that it is unlikely that a single study can uncover all aspects (e.g., cultural and structural issues and impacts) relating the TT-SET augmented with POT. I believe that it is impossible for this small research project to make great difference in the literature of POT because each study is the small contribution to the overall wealth of knowledge. Small bits of knowledge will be accumulated overtime when further research is done and adds to the understanding of the intervention process. Thus, this study is just the start of a long journey to examine the intervention. Further research may need to take into account power imbalance, students' learning outcomes, longer span of time for POT, and other cultural issues in details.

In summary, the intervention process facilitated changes in most academics' pedagogical reasoning and appeared to create an opportunity for their learning about teaching. Reflection is a factor underlying other changes (outcomes), so the intervention may have created a chance for some academics to take a reflective stance on their teaching, from which more effective teaching can result and may benefit students. TT-SET augmented with collaborative POT is for academics' reflection and by students' and peers' lenses. There are challenges to be addressed for better implementation of the intervention. With the time pressure many academics had to bear, it seems unreasonable for them to put on burden. However, it is academics' responsibility to improve teaching in order to support the Vietnamese Government's (2005) 2006-2020 project to develop well-qualified tertiary teaching. Particularly, the intervention enhanced academics' awareness of their practice which is fundamental to their action to improve practice. Besides students, the peer appeared to be a supportive source for many academics' teaching. In many cases, collaboration and collegiality were perceived to improve and learning was facilitated, so colleagues can be effective teachers to one another.

To conclude, the importance of collaboration between academics for reflection and learning can be expressed in the Vietnamese proverb that follows:

\section{"Hoc thầy không tày hoc bạn."}

(translated into English as: "Peers make better teachers.") 



\section{References}

Abd-El-Khalick, F. (2006). Preservice and experienced biology teachers' global and specific subject matter structures: Implications for conceptions of pedagogical content knowledge. Eurasia Journal of Mathematics, Science \& Technology Education, 2(1), 1-29.

Adshead, L., White, P. T., \& Stephenson, A. (2006). Introducing peer observation of teaching to GP teachers: A questionnaire study. Medical Teacher, 28(2), e68e73.

Aguirre, J., \& Speer, N. M. (2000). Examining the relationship between beliefs and goals in teacher practice. Journal of Mathematical Behaviour, 18(3), 327-356.

Algozzine, B., Beattie, J., Bray, M., Flowers, C., Gretes, J., Howley, L., . . Spooner, F. (2004). Student evaluation of college teaching: A practice in search of principles. College Teaching, 52(4), 134-141.

Ambler, T., Chavan, M., Clarke, J., \& Matthews, N. (2014). Climates of communication: Collegiality, affect, spaces and attitudes in peer review. In J. Sachs \& M. Parsell (Eds.), Peer review of learning and teaching in higher education: International perspectives (Vol. Professional learning and development in schools and higher education, pp. 67-84). New York: Springer.

Argyris, C., \& Schön, D. A. (1974). Theory in practice: Increasing professional effectiveness. San Francisco: Jossey-Bass.

Arthur, L. (2009). From performativity to professionalism: Lecturers' responses to student feedback. Teaching in Higher Education, 14(4), 441-454.

Ashwill, M. A., \& Diep, T. N. (2005). Vietnamese today: A guide to a national at a crossroads. Yarmouth, ME: Intercultural Press.

Atkinson, D. J., \& Bolt, S. (2010). Using teaching observations to reflect upon and improve teaching practice in higher education. Journal of the Scholarship of Teaching and Learning, 10(3), 1-19. 
Aultman, L. P. (2006). An unexpected benefit of formative student evaluations. College Teaching, 54(3), 251.

Ballantyne, R., Borthwick, J., \& Packer, J. (2000). Beyond student evaluation of teaching: Identifying and addressing academic staff development needs. Assessment \& Evaluation in Higher Education, 25(3), 221-236.

Bandura, A. (1977a). Self-efficacy: Toward a unifying theory of behavioral change. Psychological Review, 84, 191-215.

Bandura, A. (1977b). Social learning theory. Englewood Cliffs, NJ: Prentice-Hall.

Bandura, A. (1986). Social foundations of thought and action: A social cognitive theory. Upper Saddle River, NJ: Prentice-Hall.

Bandura, A. (1997). Self-efficacy: The exercise of control. London: W. H. Freeman.

Barnard, A., Croft, W., Irons, R., Cuffe, N., Bandara, W., \& Rowntree, P. (2011). Peer partnership to enhance scholarship of teaching: A case study. Higher Education Research \& Development, 30(4), 435-448.

Barnard, A., Nash, R., McEvoy, K., Shannon, S., Waters, C., Rochester, S., \& Bolt, S. (2015). LeaD-In: A cultural change model for peer review of teaching in higher education. Education Research \& Development, 34(1), 30-44.

Barth, M. M. (2008). Deciphering student evaluations of teaching: A factor analysis approach. Journal of Education for Business, 84(1), 40-46.

Basturkmen, H., Lowewen, S., \& Ellis, R. (2004). Teachers' stated beliefs about incidental focus on form and their classroom practices. Applied Linguistics, 25(2), 243-272.

Baxter, J., \& Eyles, J. (1997). Evaluating qualitative research in social geography: Establishing 'rigour' in interview analysis. Transactions of the Institute of British Geographers, 22(4), 505-525.

Baxter, P., \& Jack, S. (2008). Qualitative case study methodology: Study design and implementation for novice researchers. The Qualitative Report, 13(4), 544-559. 
Bell, A., \& Mladenovic, R. (2008). The benefits of peer observation of teaching for tutor development. Higher Education, 55(6), 735-752.

Bell, M. (2001). Supported reflective practice: A program of peer observation and feedback for academic teaching development. International Journal for Academic Development, 6(1), 29-39.

Bell, M. (2005). Peer observation partnerships in higher education. NSW, Australia: Higher Education Research and Development Society of Australasia.

Bell, M., \& Cooper, P. (2011). Peer observation of teaching in university departments: A framework for implementation. International Journal for Academic Development, 1-14.

Bell, M., \& Cooper, P. (2013). Peer observation of teaching in university departments: A framework for implementation. International Journal for Academic Development, 18(1), 60-73.

Belluigi, D. Z. (2013). Playing broken telephone with student feedback: The possibilities and issues of transformation within a South African case of collegial rationality model of evaluation. In C. S. Nair \& P. Mertova (Eds.), Enhancing learning and teaching through student feedback in social sciences (pp. 1-28). Oxford, UK: Chandos.

Bennett, L., \& Nair, C. S. (2010). A recipe for effective participation rates for webbased surveys. Assessment \& Evaluation in Higher Education, 35(4), 357-365.

Bennett, S., \& Barp, D. (2008). Peer observation: A case for doing it online. Teaching in Higher Education, 13(5), 559-570.

Benson, P. (2000). Autonomy as a learners' and teachers' right. In B. Sinclair, I. McGrath \& T. Lamb (Eds.), Learner autonomy, teacher autonomy: Future directions (pp. 111-117). London: Longman.

Beran, T., Violato, C., Kline, D., \& Frideres, J. (2005). The utility of student ratings of instruction for students, faculty, and administrators: A "consequential validity" study. The Canadian Journal of Higher Education, 35(2), 49-70. 
Berger, R. (2013). Now I see it, now I don't: Researcher's position and reflexivity in qualitative research. Qualitative Research, 1-16.

Blackwell, R., \& McLean, M. (1996). Peer observation of teaching and staff development. Higher Education Quarterly, 50(2), 156-171.

Bolton, G. (2010). Reflective practice: Writing and professional development ( $\left.3^{\text {rd }} \mathrm{ed}.\right)$. London: SAGE.

Borg, M. (2001). Key concepts in ELT: Teachers' beliefs. ELT Journal, 55(2), 186188.

Borg, S. (2003). Teacher cognition in language teaching: A review of research on what language teachers think, know, believe, and do. Language Teaching, 36(2), 81109.

Borton, L. (2000). Working in a Vietnamese voice. Academy of Management Executive, 14(4), 20-29.

Boud, D., Cressey, P., \& Docherty, P. (2006). Productive reflection at work: Learning for changing organisations. London: Routledge.

Boud, D., \& Walker, D. (1998). Promoting reflection in professional courses: The challenge of context. Studies in Higher Education, 23(2), 191-206.

Braun, V., \& Clarke, V. (2006). Using thematic analysis in psychology. Qualitative Research in Psychology, 3(2), 77-101.

Brinko, K. T. (1993). The practice of giving feedback to improve teaching: What is effective? The Journal of Higher Education, 64(5), 574-593.

Brockett, R. G. (2006). Self-directed learning and the paradox of choice. International Journal of Self-directed Learning, 3(2), 27-33.

Brookfield, S. (1995). Becoming a critically reflective teacher: San Francisco, CA: Jossey-Bass.

Brown, M. J. (2008). Student perceptions of teaching evaluations. Journal of Instructional Psychology, 35(2), 177-181. 
Bullock, C. D. (2003). Online collection of midterm student feedback. [Article]. New Directions for Teaching \& Learning, 2003(96), 95-102.

Bullock, D. (2011). Learner self-assessment: An investigation into teachers' beliefs. ELT Journal, 65(2), 114-125.

Bullock, S. M. (2011). Inside teacher education: Challenging prior views of teaching and learning. Rotterdam: Sense.

Burden, P. (2010). Creating confusion or creative evaluation? The use of student evaluation of teaching surveys in Japanese tertiary education. Educational Assessment, Evaluation and Accountability, 22(2), 97-117.

Buysse, V., Sparkman, K. L., \& Wesley, P. W. (2003). Communities of practice: Connecting what we know with what we do. Exceptional Children, 69(3), 263277.

Byrne, J., Brown, H., \& Challen, D. (2010). Peer development as an alternative to peer observation: A tool to enhance professional development. International Journal for Academic Development, 15(3), 215-228.

Cairns, A. M., Bissell, V., \& Bovill, C. (2013). Evaluation of a pilot peer observation of teaching scheme for chair-side tutors at Glasgow University Dental School. British Dental Journal, 214(11), 573-576.

Calderhead, J. (1996). Teachers: Beliefs and knowledge. In D. C. Berliner \& R. C. Calfee (Eds.), Handbook of educational psychology (pp. 709-725). New York: Simon \& Schuster Macmillan.

Canrinus, E. T., Helms-Lorenz, M., Beijaard, D., Buitink, J., \& Hofman, A. (2012). Self-efficacy, job satisfaction, motivation and commitment: Exploring the relationships between indicators of teachers' professional identity. Journal of Psychology of Education, 27(1), 115-132.

Carroll, C., \& O'Loughlin, D. (2014). Peer observation of teaching: Enhancing academic engagement for new participants. Innovations in Education and Teaching International, 51(4), 446-456. 
Centre for Academic Development. Feedback questions. Retrieved 15 October, 2012, from http://www.cad.vuw.ac.nz/wiki/index.php/Feedback_Questions

Chism, N. V. N. (2007). Peer review of teaching: A sourcebook ( $2^{\text {nd }}$ ed.). Bolton, MA: Anker.

Clancey, W. J. (1997). Situated cognition: On human knowledge and computer representation. Cambridge, UK: Cambridge University Press.

Clift, J., Hall, C., \& Turner, I. (1989). Establishing the validity of a set of summative teaching performance scales. Assessment and Evaluation in Higher Education, 14(3), 193-206.

Cochran-Smith, M., \& Lytle, S. L. (1999). Relationships of knowledge and practice: Teacher learning in communities. Review of Research in Education, 24, 249305 .

Cohen, P. A. (1980). Effectiveness of student-rating feedback for improving college instruction: A meta-analysis of findings. Research in Higher Education, 13(4), 321-341.

Coleman, H. (2010). The English language in development. London: British Council Retrieved from https://www.teachingenglish.org.uk/sites/teacheng/files/UK011-EnglishLanguage-Development.pdf.

Colton, A. B., \& Sparks-Langer, G. M. (1993). A conceptual framework to guide the development of teacher reflection and decision making. Journal of Teacher Education, 44(1), 45-54.

Convery, A. (1998). A teacher's response to 'reflection-in-action'. Cambridge Journal of Education, 28(2), 197-205.

Corbin, J., \& Strauss, A. (2008). Basics of qualitative research: Techniques and procedures for developing grounded theory ( $3^{\text {rd }}$ ed.). Thousand Oaks, CA: SAGE. 
Cosh, J. (1998). Peer observation in higher education: A reflective approach. Innovations in Education and Training International, 35(2), 171-176.

Cosh, J. (1999). Peer observation: A reflective model. ELT Journal, 53(1), 22-27.

Creswell, J. W. (2009). Research design: Qualitative, quantitative, and mixed methods approaches ( $3^{\text {rd }}$ ed.). Thousand Oaks, CA: SAGE.

Creswell, J. W., \& Miller, D. L. (2000). Determining validity in qualitative inquiry. Theory into Practice, 39(3), 124-130.

Cutcliffe, J. R. (2003). Reconsidering reflexivity: Introducing the case for intellectual entrepreneurship. Qualitative Health Research, 13(1), 136-148.

Darling-Hammond, L. (2006). Constructing 21st-century teacher education. Journal of Teacher Education, 57(3), 300-314.

Denzin, N. K., \& Lincoln, Y. S. (2011). The SAGE handbook of qualitative research ( $4^{\text {th }}$ ed.). Thousand Oak,CA: SAGE.

Dewey, J. (1910). How we think. Boston: D. C. Health \& Co. Publishers.

Dewey, J. (1997). How we think (Original work published 1910, Reprint Unabridged ed.). Mineola, NY: Dover.

Donald, C., Blake, A., Girault, I., Datt, A., \& Ramsay, E. (2009). Approaches to learning design: Past the head and the hands to the HEART of the matter. Distance Education, 30(2), 179-199.

Donnelly, R. (2007). Perceived impact of peer observation of teaching in higher education. International Journal of Teaching and Learning in Higher Education, 19(2), 117-129.

Douglas, J., \& Douglas, A. (2006). Evaluating teaching quality. Quality in Higher Education, 12(1), 3-13.

Dresel, M., \& Rindermann, H. (2011). Counseling university instructors based on student evaluations of their teaching effectiveness: A multilevel test of its 
effectiveness under consideration of bias and unfairness variables. Research in Higher Education, 52(7), 717-737.

Dung, N. K., \& Mcinnis, C. (2002). The possibility of using student evaluations in Vietnamese higher education. Quality in Higher Education, 8(2), 151-158.

Ecclestone, K. (1996). The reflective practitioner: Mantra or a model for emancipation. Studies in the Education of Adults, 28(2), 146-161.

Eddy, P. L., \& Garza Mitchell, R. L. (2012). Faculty as learners: Developing thinking communities. Innovative Higher Education, 37(4), 283-296.

Engin, M., \& Priest, B. (2014). Observing teaching: A lens for self-reflection. Journal of Perspectives in Applied Academic Practice, 2(2), 2-9.

Ertmer, P. A. (2005). Teacher pedagogical beliefs: The final frontier in our quest for technology integration? Educational Technology Research and Development, 53(4), 25-39.

Evans, R. (2011). Case study method in sustainability research. In A. Franklin \& P. Blyton (Eds.), Researching sustainability: A guide to social science methods, practice and engagement (pp. 54-70). New York: Earthscan.

Fernandez, C. E., \& Yu, J. (2007). Peer review of teaching. The Journal of Chiropractic Education, 21(2), 154-161.

Finn, K., Chiappa, V., Puig, A., \& Hunt, D. P. (2011). How to become a better clinical teacher: A collaborative peer observation process. Medical Teacher, 32(2), 151155.

Forde, C., McMahon, M., McPhee, A. D., \& Patrick, F. (2006). Professional development, reflection and enquiry. London: SAGE.

Freedman, S. (2009). Collegiality matters: How do we work with others? Paper presented at the Charleston Library Conference, Purdue University. http://dx.doi.org/10.5703/1288284314771 
Fullerton, H. (1993). Observation of teaching : Guidelines for observers and observed. In S. Brown, G. Jone \& S. Rawnsley (Eds.), Observing teaching (pp. 77-90). Birmingham: SEDA.

Fullerton, H. (2003). Observation of teaching. In H. Fry, S. Ketteridge \& S. Marshall (Eds.), A handbook for teaching and learning in higher education $\left(2^{\text {nd }}\right.$ ed., pp. 226-241). London: Kogan Page.

Gagne, M., \& Deci, E. L. (2005). Self-determination theory and work motivation. Journal of Organizational Behavior, 26(3), 331-362.

Given, L. M., \& Saumure, K. (2008). Trustworthiness. In L. M. Given (Ed.), The Sage encyclopedia of qualitative research methods (pp. 896-897). Thousand Oaks, CA: SAGE.

Gosling, D. (2002). Models of peer observation of teaching. Retrieved from http://www.heacademy.ac.uk/resources/detail/resource_database/id200_Models _of_Peer_Observation_of_Teaching.

Gosling, D. (2009). A new approach to peer review of teaching. In D. Gosling \& K. M. O'Connor (Eds.), Beyond the peer observation of teaching (pp. 7-15). London: SEDA.

Gosling, D. (2014). Collaborative peer-supported review of teaching. In J. Sachs \& M. Parsell (Eds.), Peer review of learning and teaching in higher education: International perspectives (Vol. Professional learning and development in schools and higher education, pp. 13-32). New York: Springer.

Grant, C. A., \& Zeichner, K. M. (2000). On becoming a reflective teacher. In J. M. Iseke-Barnes \& N. N. Wane (Eds.), Equity in schools and society (pp. 25-42). Toronto, Ontario: Canadian Scholars' Press.

Gray, D. E. (2014). Doing research in the real world (3 ${ }^{\text {rd }}$ ed.). London: Sage.

Greenwald, A. G., \& Gillmore, G. M. (1997). Grading leniency is a removable contaminant of student ratings. American Psychologist, 52(11), 1209-1217. 
Grimmett, P., Mackinnon, A., Erickson, G., \& Riecken, T. (1990). Reflective practice in teacher education. In R. T. Clift, W. R. Houston \& M. C. Pugach (Eds.), Encouraging reflective practice in education: An analysis of issues and programs (pp. 20-37). New York: Teachers College Press.

Grünbaum, N. N. (2007). Identification of ambuguity in the case study research typology: What is a unit of analysis? Qualitative Market Research: An International Journal, 10(1), 78-97.

Guba, E. G., \& Lincoln, Y. S. (1994). Competing paradigms in qualitative research. In N. K. Denzin \& Y. S. Lincoln (Eds.), Handbook of qualitative research (pp. 105117). Thousand Oaks, CA: SAGE.

Hafler, J. P. (2002). Observing, developing, and reflecting on residents' teaching strategies. In J. C. Edwards, J. A. Friedland \& R. Bing-You (Eds.), Residents' teaching skills (pp. 66-80). New York: Springer.

Haigh, N. (2005). Everyday conversation as a context for professional learning and development. International Journal for Academic Development, 10(1), 3-16.

Hallinger, P. (2010). Using faculty evaluation to improve teaching quality: A longitudinal case study of higher education in Southeast Asia. Educational Assessment, Evaluation and Accountability, 22(4), 253-274.

Hammersley-Fletcher, L., \& Orsmond, P. (2004). Evaluating our peers: Is peer observation a meaningful process? Studies in Higher Education, 29(4), 489-503.

Hammersley-Fletcher, L., \& Orsmond, P. (2005). Reflecting on reflective practices within peer observation. Studies in Higher Education, 30(2), 213-224.

Haney, J. J., Lumpe, A. T., \& Czerniak, C. M. (2003). Constructivist beliefs about the science classroom learning environment: Perspectives from teachers, administrators, parents, community members, and students. School Science and Mathematics, 103(8), 366-377. 
Hao, N. T. (2013). Student ratings in Vietnam higher education: How are instructors' reactions? International Journal of Innovative Management, Information \& Production, 4(1), 99-109.

Hargreaves, A., \& Dawe, R. (1990). Paths of professional development: Contrived collegiality, collaborative culture, and the case of peer coaching. Teaching and Teacher Education, 6(3), 227-241.

Hargreaves, A., \& Fink, D. (2003). Sustaining leadership. Phi Delta Kappan, 84(9), 693-700.

Harper, F., \& Nicolson, M. (2013). Online peer observation: Its value in teacher professional development, support and well-being. International Journal for Academic Development, 18(3), 264-275.

Harris, K.-L., Farrell, K., Bell, M., Devlin, M., \& James, R. (2008). Peer review of teaching in Australian higher education: A handbook to support institutions in developing and embedding effective policies and practices Retrieved from http://www.cshe.unimelb.edu.au/research/teaching/docs/PeerReviewHandbook _eVersion.pdf

Harrison, J. (2008). Professional learning and the reflective practitioner. In S. Dymoke \& J. Harrison (Eds.), Reflective teaching and learning (pp. 7-44). London: SAGE.

Harvey, L. (2003). Student feedback. Quality in Higher Education, 9(1), 3-20.

Hattie, J., \& Timperley, H. (2007). The power of feedback. Review of Educational Research, 77(1), 81-112.

Hatton, N., \& Smith, D. (1995). Reflection in teacher education: Towards definition and implementation. Teaching and Teacher Education, 11(1), 33-49.

Hatzipanagos, S., \& Lygo-Baker, S. (2006a). Teaching observations: A meeting of minds? International Journal of Teaching and Learning in Higher Education, 17(2), 97-105. 
Hatzipanagos, S., \& Lygo-Baker, S. (2006b). Teaching observations: Promoting development through critical reflection. Journal of Further and Higher Education, 30(4), 421-431.

Hénard, F. (2010). Learning our lesson: Review of quality teaching in higher education doi:10.1787/9789264079281-en

Hendry, G. D., Bell, A., \& Thomson, K. (2013). Learning by observing a peer's teaching situation. International Journal for Academic Development, 1-12.

Hendry, G. D., \& Oliver, G. R. (2012). Seeing is believing: The benefits of peer observation. Journal of University Teaching \& Learning Practice, 9(1), 1-9.

Hiep, P. H. (2001). Teacher development: A real need for English departments in Vietnam. English Teaching Forum, 39(4). Retrieved from http://americanenglish.state.gov/files/ae/resource_files/01-39-4-f.pdf

Hoa, N. T. M. (2008). Mentoring beginning EFL teachers at tertiary level in Vietnam. The Asian EFL Journal, 10(1), 111-132.

Hoa, N. T. M. (2013). Peer mentoring: A way forward for supporting preservice EFL teachers psychosocially during the practicum. Australian Journal of Teacher Education, 38(7), 31-44.

Hofman, J. E., \& Kremer, L. (1983). Course evaluation and attitudes toward college teaching. Higher Education, 12(6), 681-690.

Hofstede, G. (1997). Cultures and organizations: Software of the mind. The United State of America: McGraw-Hill.

Horner, S. L., Bhattacharyya, S., \& O'Connor, E. A. (2008). Modeling: It's more than just imitation. Childhood Education, 84(4), 219-222.

Horsburgh, D. (2003). Evaluation of qualitative research. Journal of Clinical Nursing, $12,307-312$. 
Hounsell, D. (2009). Evaluating courses and teaching. In H. Fry, S. Ketteridge \& S. Marshall (Eds.), A handbook for teaching and learning in higher education: Enhancing academic practice (pp. 1998-1211). New York: Routledge.

Hu, G. (2002). Potential cultural resistance to pedagogical imports: The case of communicative language teaching in China. Language, Culture and Curriculum, 15(2), 93-105.

Hu, G. (2005). Professional development of secondary EFL teachers: Lessons from China. Teachers College Record, 107(4), 654-705.

Huong, P. T. (2014). Quality culture in Vietnamese universities: a multiple case study of quality assurance and quality culture of business English undergraduate programmes at three universities in Vietnam. PhD Thesis, Victoria University of Wellington, New Zealand.

Hutchings, P. (1996). The peer review of teaching: Progress, issues and prospects. Innovative Higher Education, 20(4), 221-234.

Iqbal, I. (2013). Academics' resistance to summative peer review of teaching: Questionable rewards and the importance of student evaluations. Teaching in Higher Education, 18(5), 557-569.

Jamieson, N. L. (1993). Understanding Vietnam. Berkeley, CA: University of Carlifornia Press.

Jensen, D. (2008a). Confirmability. In L. M. Given (Ed.), The Sage encyclopedia of qualitative research methods (pp. 113). Thousand Oaks, CA: SAGE.

Jensen, D. (2008b). Credibility. In L. M. Given (Ed.), The Sage encyclopedia of qualitative research methods (pp. 139-140). Thousand Oaks, CA: SAGE.

Jensen, D. (2008c). Transferability. In L. M. Given (Ed.), The Sage encyclopedia of qualitative research methods (pp. 887). Thousand Oaks, CA: SAGE.

Johnson, K. E. (2006). The sociocultural turn and its challenges for second language teacher education. TESOL Quarterly, 40(1), 235-257. 
Johnson, K. E., \& Golombek, P. R. (2002). Teachers' narrative inquiry as professional development. Cambridge: Cambridge University Press.

Jones, E. (2007). A portfolio for assessment of the practice of the special education resource teachers. $\mathrm{PhD}$ Thesis, Victoria University of Wellington, New Zealand.

Jones, E. (2010). A professional practice portfolio for quality learning. Higher Education Quarterly, 64(3), 292-312.

Kagan, D. M. (1992). Implications of research on teacher belief. Educational Psychologist, 27(1), 65-90.

Kane, R., Sandretto, S., \& Heath, C. (2002). Telling half the story: A critical review of research on the teaching beliefs and practices of university academics. Review of Educational Research, 72(2), 177-228.

Kelchtermans, G. (2009). Who I am in how I teach is the message: Self-understanding, vulnerability and reflection. Teachers and Teaching: Theory and Practice, 15(2), 257-272.

Kember, D., Leung, D. Y. P., \& Kwan, K. P. (2002). Does the use of student feedback questionnaires improve the overall quality of teaching? Assessment \& Evaluation in Higher Education, 27(5), 411-426.

Kemp, R., \& Gosling, D. (2012). Peer observation of teaching Retrieved 18 May 2015, from https://www.academia.edu/2233137/Peer_observation_of_teaching

Knight, P. J. M. (2006). The professional learning of teachers in higher education. Studies in Higher Education, 31(3), 319-339.

Knowles, J. G. (1993). Life-history accounts as mirrors: A practical avenue for the conceptualization of reflection in teacher education. In J. Calderhead \& P. Gates (Eds.), Conceptualizing reflection in teacher development (pp. 70-92). London: Falmer Press.

Knowles, M. S. (1975). Self-directed learning: a guide for learners and teachers. New York: Cambridge, The Adult Education Company 
Knowles, M. S., Holton, E. F., \& Swanson, R. A. (2005). The adult learner: The definitive classic in adult education and human resource development $\left(6^{\text {th }} \mathrm{ed}\right.$.). California: Elsevier.

Korthagen, F. (2001). Linking practice and theory: The pedagogy of realistic teacher education. Mahwah, NJ: Lawrence Erlbaum.

Korthagen, F., \& Lagerwerf, B. (2001). Teachers' professional learning: How does it work? In F. Korthagen (Ed.), Linking practice and theory: The pedagogy of realistic teacher education (pp. 175-206). Mahwah, NJ: Lawrence Erlbaum.

Korthagen, F. A. J. (2010). Situated learning theory and the pedagogy of teacher education: Towards an integrative view of teacher behavior and teacher learning. Teaching and Teacher Education, 26(1), 98-106.

LaBoskey, V. (1993). A conceptual framework for reflection in preservice teacher education. In J. Calderhead \& P. Gates (Eds.), Conceptualizing reflection in teacher development (pp. 23-38). London: Falmer Press.

LaBoskey, V. K. (2004). The methodology of self-study and its theoretical underpinnings. In J. J. Loughran, M. L. Hamilton, V. K. LaBoskey \& T. Russell (Eds.), International handbook of self-study of teaching and teacher education practices (pp. 817-869). Dordrecht: Kluwer.

Lalonde, R. N., Hynie, M., Pannu, M., \& Tatla, S. (2004). The role of culture in interpersonal relationships: Do second generation South Asian Canadians want a traditional partner? Journal of Cross-Cultural Psychology, 35(5), 503-524.

Lamb, T. (2008). Learner autonomy and teacher autonomy: Synthesising an agenda. In T. Lamb \& H. Reinders (Eds.), Learner and teacher autonomy: Concepts, realities, and responses (pp. 269-284). Amsterdam: John Benjamins.

Lang, J. W. B., \& Kersting, M. (2007). Regular feedback from student ratings of instruction: Do college teachers improve their ratings in the long run? Instructional Science, 35(3), 187-205. 
Lasley, T. (1992). Promoting teacher reflection. Journal of Staff Development, 13(1), 24-29.

Lee, J. C.-K., Huang, Y. X., \& Zhong, B. (2012). Friend or foe: The impact of undergraduate teaching evaluation in China. Higher Education Review, 44(2), 255.

Lincoln, Y. S., \& Guba, E. G. (1985). Naturalistic inquiry. Newbury Park, CA: Sage.

Little, D. (1995). Learning as dialogue: The dependence of learner autonomy on teacher autonomy. System, 23(2), 175-181.

Lomas, L., \& Nicholls, G. (2005). Enhancing teaching quality through peer review of teaching. Quality in Higher Education, 11(2), 137-149.

Long, V. T., \& Hoa, N. T. M. (2010). Critical friends group for EFL teacher professional development. ELT Journal, 64(2), 205-213.

Loughran, J. (1995). Practising what I preach: Modelling reflective practice to student teachers. Research in Science Education, 25(4), 431-451.

Loughran, J. (2002). Effective reflective practice: In search of meaning in learning about teaching. Journal of Teacher Education, 53(1), 33-43.

Lu, J., Jiang, X., Yu, H., \& Li, D. (2014). Building collaborative structures for teachers' autonomy and self-efficacy: the mediating role of participative management and learning culture. School Effectiveness and School Improvement: An International Journal of Research, Policy and Practice.

MacKeracher, D. (2004). Making sense of adult learning ( $2^{\text {nd }}$ ed.). Toronto, Buffalo: University of Toronto Press.

MacKinnon, M. M. (2001). Using observational feedback to promote academic development. International Journal for Academic Development, 6(1), 21-28.

Mai, N. P., Terlouw, C., \& Pilot, A. (2005). Cooperative learning vs Confucian heritage culture's collectivism: Confrontation to reveal some cultural conflicts and mismatch. Asia Europe Journal, 3(3), 403-419. 
Mansfield, C. F., \& Woods-McConney, A. (2012). “I didn’t always perceive myself as a science person": Examining efficacy for primary science teaching. Australian Journal of Teacher Education, 37(10), 37-52.

Marcos, J. J. M., Sánchez, E., \& Tillema, H. (2008). Teachers reflecting on their work: articulating what is said about what is done. Teachers and Teaching: theory and practice, $14(2), 95-114$.

Marsh, H. W. (1982). SEEQ: A reliable, valid, and useful instrument for collecting students' evaluations of university teaching. British Journal of Educational Psychology, 52(1), 77-95.

Marsh, H. W. (1987). Students' evaluations of university teaching: Research findings, methodological issues, and directions for future research. International Journal of Educational Research, 11(3), 253-388.

Marsh, H. W., \& Roche, L. A. (1993). The use of students' evaluations and an individually structured intervention to enhance university teaching effectiveness. American Educational Research Journal, 30(1), 217-251.

Marsh, H. W., \& Roche, L. A. (1997). Making students' evaluations of teaching effectiveness effective: The critical issues of validity, bias, and utility. American Psychologist, 52(11), 1187-1197.

Marshall, B. (2004). Learning from the Academy: From peer observation of teaching to peer enhancement of learning and teaching. Journal of Adult Theological Education, 1(2), 185-204.

Marshall, M. N. (1996). Sampling for qualitative research. Family Practice, 13(6), $522-525$.

Martin, G. A., \& Double, J. M. (1998). Developing higher education teaching skills through peer observation and collaborative reflection. Innovations in Education and Training International, 35(2), 161-169.

Marton, F., \& Booth, S. (1997). Learning and awareness. Mahwah, NJ: Lawrence Erlbaum. 
Maxwell, J. A. (2005). Qualitative research design: An interative approach ( $\left.2^{\text {nd }} \mathrm{ed}.\right)$. Thousand Oaks, CA: SAGE.

McAlpine, L., \& Weston, C. (2000). Reflection: Issues related to improving professors' teaching and students' learning. Instructional Science, 28(5), 363385.

McArthur, J. (2011). Reconsidering the social and economic purposes of higher education. Higher Education Research \& Development, 30(6), 737-749.

McGill, I., \& Beaty, L. (1995). Action learning: A guide for professional, management, and educational development ( $2^{\text {nd }}$ ed.). London: Kogan Page

McIntyre, D. (1993). Theory, theorising and reflection in initial teacher education. In J. Calderhead \& P. Gates (Eds.), Conceptualizing reflection in teacher development (pp. 39-52). London: Falmer Press.

McKeachie, W. J. (1997). Student ratings: The validity of use. American Psychologist, 52(11), 1218-1225.

McLeod, S. A. (2014). Cognitive dissonance. Retrieved from www.simplypsychology.org/cognitive-dissonance.html

McMahon, T., Barrett, T., \& O’Neill, G. (2007). Using observation of teaching to improve quality: Finding your way through the muddle of competing conceptions, confusion of practice and mutually exclusive intentions. Teaching in Higher Education, 12(4), 499-511.

McPherson, M. A., \& Kennedy, P. (2006). Determinants of how students evaluate teachers. Journal of Economic Education, 37(1), 3-20.

Merriam, S. B. (1988). Case study research in education: A qualitative approach. San Francisco, CA: Jossey-Bass.

Merriam, S. B. (1998). Qualitative research and case study applications in education. San Francisco: Jossey-Bass. 
Merriam, S. B. (2001). Qualitative research and case study applications in education ( $2^{\text {nd }}$ ed.). San Francisco, CA: Jossey-Bass.

Merriam, S. B. (2009). Qualitative research: A guide to design and implementation. San Francisco, CA: Jossey-Bass.

Mertens, D. M. (2005). Research and evaluation in education and psychology: Integrating diversity with quantitative, qualitative, and mixed methods. Thousand Oaks, CA: SAGE.

Mezirow, J. (1991). Transformative dimensions of adult learning. San Fransisco, CA: Jossey-Bass.

Mezirow, J. (2003). How critical reflection triggers transformative learning. In P. Jarvis \& C. Griffin (Eds.), Adult and continuing education: Major themes in education (Vol. Teaching, Learning, and Research, pp. 199-213). London: Routledge.

Miles, M. B., \& Huberman, A. M. (1994). Qualitative data analysis: An expanded sourcebook (2 $2^{\text {nd }}$ ed.). Thousand Oaks, CA: SAGE.

Moore, S., Walsh, G., \& Risquez, A. (2007). Teaching at college and university: Effective strategies and key principles. Maidenhead, Berkshire: Open University Press.

Moore, T. (2006). Teacher evaluations and grades: Additional evidence. Journal of American Academy of Business, 9(2), 58-62.

Moore, T. J., \& Asay, S. M. (2013). Family resource management. Thousand Oaks, CA: SAGE.

Murray, H. G. (1997). Does evaluation of teaching lead to improvement of teaching? International Journal for Academic Development, 2(1), 8-23.

Nair, C. S., \& Mertova, P. (2013). Emerging trends and approaches in the student voice in the social sciences. In C. S. Nair \& P. Mertova (Eds.), Enhancing learning and teaching through student feedback in social sciences (pp. 201-208). Oxford, UK: Chandos. 
Narasimhan, K. (2001). Improving the climate of teaching sessions: The use of evaluations by students and instructors. Quality in Higher Education, 7(3), 179190.

Nespor, J. (1987). The role of beliefs in the practice of teaching. Journal of Curriculum Studies, 19(4), 317-328.

Nunan, D. (2003). The impact of English as a global language on educational policies and practices in the Asia-Pacific Region. TESOL Quarterly, 37(4), 589-613.

Osterman, K. F., \& Kottkamp, R. B. (1993). Reflective practice for educators: Improving schooling through professional development. Newbury Park, CA: Corwin Press.

Pajares, M. F. (1992). Teachers beliefs and educational research: Cleaning up a messy construct. Review of Educational Research, 62(3), 307-332.

Palmer, P. J. (1998). The courage to teach: Exploring the inner landscape of a teacher's life (1 $1^{\text {st }}$ ed.). San Francisco, CA: Jossey-Bass.

Patton, M. Q. (1990). Qualitative evaluation and research methods. Beverly Hills, CA: Sage.

Patton, M. Q. (2002). Qualitative research and evaulation methods. Thousand Oaks, CA: SAGE.

Paulsen, M. R., \& Feldman, K. A. (1995). Taking teaching seriously: Meeting the challenge of instructional improvement (ASHE-ERIC Higher Education Reports ed.). Washington, DC: The George Washington University.

Pedro, J. (2006). Taking reflection into the real world of teaching. Kappa Delta Pi Record, 42(3), 129-132.

Peel, D. (2005). Peer observation as a transformatory tool? Teaching in Higher Education, 10(4), 489-504. 
Pendergast, D., \& Garvis, S. (2011). Pre-service student-teacher self-efficacy beliefs: An insight into the making of teachers. Australian Journal of Teacher Education, 36(12), 46-58.

Penny, A. R. (2004). Responding to students' views about university teaching: the experience of teachers in Jamaica. International Journal of Educational Development, 24(6), 667-676.

Penny, A. R., \& Coe, R. (2004). Effectiveness of consultation on student ratings feedback: A meta-analysis. Review of Educational Research, 74(2), 215-253.

Phinney, J. S., Ong, A., \& Madden, T. (2000). Cultural values and intergenerational value discrepancies in immigrant and non-immigrant families. Child Development, 71(2), 528-539.

Phuong, L. T. A., \& Vásquez, C. (2011). Feedback in teacher education: Mentor discourse and intern perceptions. Teacher Development: An International Journal of Teachers' Professional Development, 15(4), 453-470.

Pollard, A. (2005). Reflective teaching ( $2^{\text {nd }}$ ed.). London: Continuum.

Potter, M., Haynes, S., Heyl, K., Phillips, K. R., Pozzi, R., Stroup, L., \& Tull, T. (2011). Peer observation of teaching: A case for culture change. TeacherScholar: The Journal of the State Comprehensive University, 3(1), 29-38.

Pressick-Kilborn, K., \& te Riele, K. (2008). Learning from reciprocal peer observation: A collaborative self-study. Studying Teacher Education: A Journal of Self-Study of Teacher Education Practices, 4(1), 61-75.

Ramsden, P. (1991). A performance indicator of teaching quality in higher education: The course experience questionnaire. Studies in Higher Education, 16(2), 129150.

Ramsden, P. (2003). Learning to teach in higher education ( $2^{\text {nd }}$ ed.). London: RoutledgeFalmer. 
Reid, K. (2010). An evaluation of an internal audit on student feedback within a British university: A quality enhancement process. Quality Assurance in Education, 18(1), 47-63.

Reis, H. T., Collins, W. A., \& Berscheid, E. (2000). The relationship context of human behavior and development. Psychological Bulletin, 126(6), 844-872.

Richards, J. C. (2011). Competence and performance in language teaching. Cambridge: Cambridge University Press.

Richardson, J. T. E. (2005). Instruments for obtaining student feedback: A review of the literature. Assessment \& Evaluation in Higher Education, 34(4), 387-415.

Rindermann, H., Kohler, J., \& Meisenberg, G. (2007). Quality of instruction improved by evaluation and consultation of instructors. International Journal for Academic Development, 12(2), 73-85.

Riniolo, T., Johnson, C., Sherman, T., \& Misso, J. (2006). Hot or not: Do professors perceived as physically attractive receive higher student evaluations? The Journal of General Psychology, 133(1), 19-35.

Roehrig, G. H., \& Kruse, R. A. (2005). The role of teachers' beliefs and knowledge in the adoption of a reform- based curriculum. School Science and Mathematics, $105(8), 412-422$.

Rotem, A., \& Glasman, N. S. (1979). On the effectiveness of students' evaluative feedback to univeristy instructors. Review of Educational Research, 49(3), 497511.

Rowley, J. (2003). Designing student feedback questionnaires. Quality Assurance in Education, 11(3), 142-149.

Ryan, R. M., \& Deci, E. L. (2000a). Intrinsic and extrinsic motivations: Classic definitions and new directions. Contemporary Educational Psychology, 25(1), 54-67. 
Ryan, R. M., \& Deci, E. L. (2000b). Self-determination theory and the facilitation of intrinsic motivation, social development, and well being. American Psychologist, 55(1), 68-78.

Schön, D. A. (1983). The reflective practitioner: How professionals think in action. New York: Basic Books.

Schön, D. A. (1987). Educating the reflective practitioner: Toward a new design for teaching and learning in the professions. San Francisco: Jossey-Bass.

Schuck, S. (2011). Resisting complacency: My teaching through an outsider's eyes. In S. Schuck \& P. Pereira (Eds.), What counts in teaching mathematics: Adding value to self and content (pp. 61-76). New York: Springer.

Schuck, S., Aubusson, P., \& Buchanan, J. (2008). Enhancing teacher education practice through professional learning conversations. European Journal of Teacher Education, 31(2), 215-227.

Schwarzer, R., \& Hallum, S. (2008). Perceived teacher self-efficacy as a predictor of job stress and burnout: Mediation analyses. Apllied Psychology, 57, 152-171.

Seldin, P. (1989). Using student feedback to improve teaching. In A. F. Lucas (Ed.), The department chairperson's role in enhancing college teaching (New directions for teaching and learning ed., Vol. 37, pp. 89-97). San Francisco: Jossey-Bass.

Seldin, P. (1993). The use and abuse of student ratings of professors. The Chronicle of Higher Education, 39(46), A40.

Shamim, F. (2011). English as the language for development in Pakistan: Issues, challenges and possible solutions. In H. Coleman (Ed.), Dreams and realities: Developing countries and the English language (pp. 291-309). London, UK: British Council.

Short, P. M. (1994). Defining teacher empowerment. Education, 114(4), 488-492.

Shortland, S. (2004). Peer observation: A tool for staff development or compliance? Journal of Further and Higher Education, 28(2), 219-228. 
Shortland, S. (2010). Feedback within peer observation: Continuing professional development and unexpected consequences. Innovations in Education and Teaching International, 47(3), 295-304.

Shulman, L. S. (1987). Knowledge and teaching: Foundations of the new reform. Harvard Educational Review, 57(1), 1-21.

Shulman, L. S. (2007). Practical wisdom in the service of professional practice. Educational Researcher, 36(9), 560-563.

Skaalvik, E. M., \& Skaalvik, S. (2014). Teacher self-efficacy and perceived autonomy: Relations with teacher engagement, job satisfaction, and emotional exhaustion. Psychological Reports: Employment Psychology \& Marketing, 114(1), 68-77.

Smith, C. (2008). Building effectiveness in teaching through targeted evaluation and response: Connecting evaluation to teaching improvement in higher education. Assessment \& Evaluation in Higher Education, 33(5), 517-533.

Smith, D. B. (1991). Teacher decision-making in the ESL classroom: The influence of theory, beliefs, perceptions and context. $\mathrm{PhD}$ Thesis, University of British Columbia, Canada.

Smith, D. B. (1996). Teacher decision making in the adult ESL classroom. In D. Freeman \& J. C. Richards (Eds.), Teacher learning in language teaching (pp. 197-216). Cambridge, UK: Cambridge University Press.

Smith, R., \& Erdoğan, S. (2008). Teacher-learner autonomy: Programme goals and student-teacher constructs. In T. Lamb \& H. Reinders (Eds.), Learner and teacher autonomy: Concepts, realities, and responses (pp. 84-101). Amsterdam: John Benjamins.

Sparks-Langer, G. M., \& Colton, A. B. (1991). Synthesis of research on teachers' reflective thinking. Educational Leadership, 48(6), 37-44.

Spencer, D. (2014). Was Moses peer observed? The ten commandments of peer observation of teaching. In J. Sachs \& M. Parsell (Eds.), Peer review of learning 
and teaching in higher education: International perspectives (pp. 183-199). London: Springer.

Spencer, P. A., \& Flyr, M. L. (1992). The formal evaluation as an impetus to classroom change: Myth or reality? (ERIC Document Reproduction Service No. ED 349 053). Retrieved from http://eric.ed.gov/?id=ED349053

Spooren, P., \& Mortelmans, D. (2006). Teacher professionalism and student evaluation of teaching: Will better teachers receive higher ratings and will better students give higher ratings? Educational Studies, 32(2), 201-214.

Stake, R. E. (1995). The art of case study research. Thousand Oaks, CA: Sage.

Stake, R. E. (2006). Multiple Case Study Analysis. New York: The Guilford Press.

Stake, R. E. (2010). Qualitative research: Studying how things work. New York: The Guilford Press.

Starkey, L. (2010a). Digital saviours: Digitally able secondary school teachers in their first year of teaching. $\mathrm{PhD}$ Thesis, Victoria University of Wellington, New Zealand.

Starkey, L. (2010b). Teachers' pedagogical reasoning and action in the digital age. Teachers and Teaching: Theory and Practice, 16(2), 233-244.

Stein, S., Spiller, D., Terry, S., Harris, T., Deaker, L., \& Kennedy, J. (2012). Unlocking the impact of tertiary teachers' perceptions of student evaluations of teaching (pp. 1-281). Wellington.

Stringer, E. T. (2007). Action research (3 ${ }^{\text {rd }}$ ed.). Thousand Oaks, CA: Sage.

Stronge, J. H., \& Tucker, P. D. (1999). The politics of teacher evaluation: A case study of new system design and implementation. Journal of Personnel Evaluation in Education, 13(4), 339-359.

Sullivan, P. B., Buckle, A., Nicky, G., \& Atkinson, S. H. (2012). Peer observation of teaching as a faculty development tool. BMC Medical Education, 12(26), 1-6. 
Suong, N. T. T. (2008). Peer debriefing. In L. M. Given (Ed.), The Sage encyclopedia of qualitative research methods (pp. 605). Thousand Oaks, CA: SAGE.

Sword, W. (1999). Accounting for presence of self: reflections on doing qualitative research. Qualitative Health Research, 9, 270-278.

Taggart, G. L., \& Wilson, A. P. (2005). Promoting reflective thinking in teachers: 50 action strategies. Thousand Oaks, CA: Corwin Press.

Tenenberg, J. (2016). Learning through observing peers in practice. Studies in Higher Education, 41(4), 756-773.

Thang, T. D. (2013). Confucian values and school leadership in Vietnam. PhD Thesis, Victoria University of Wellington, New Zealand.

The Prime Minister. (2008). The Prime Minister's decision 1400/QĐ-TTg Teaching and learning foreign languages in the national education system from 2008 to 2020 project. Hanoi: Vietnamese Government.

The Vietnamese Government. (2005). Government resolution 14/2005/NQ-CP on substantial and comprehensive reforms of Vietnam's tertiary education in the 2006-2020 period. Hanoi: Author.

The World Bank. (2000). Higher education in developing countries (Report of the Task Force on Higher Education and Society ed.). Washington, DC: The World Bank.

Thomas, D. R. (2006). A general inductive approach for analyzing qualitative evaluation data. American Journal of Evaluation, 27(2), 237-246.

Thuy, D. T. P. (2012). Beginning teachers' observation of peers - the values, limitations, and suggestions from the perspectives of the insiders. Master of Arts Thesis, Hanoi University of Languages and International Studies, Vietnam.

Tran, L., Marginson, S., Do, H., Do, Q., Le, T., Nguyen, N., . . Nguyen, H. (2014). Higher education in Vietnam: Flexibility, mobility and practicality in the global knowledge economy. Hampshire, UK: Palgrave Macmillan. 
Trujillo, J. M., DiVall, M. V., Barr, J., Gonyeau, M., Amburgh, J. A. V., Matthews, S. J., \& Qualters, D. (2008). Development of a peer teaching-assessment program and a peer observation and evaluation tool. American Journal of Pharmaceutical Education, 72(6), 1-9.

Tschannen-Moran, M., \& Hoy, A. W. (2007). The differential antecedents of selfefficacy beliefs of novice and experienced teachers. Teaching and Teacher Education, 23(6), 944-956.

Tuong, N. H. (2002). Vietnam: Cultural background for ESL/EFL teachers. Review of Vietnamese Studies, 2(1), 1-6.

Tuyet, T. T. (2013). Is the learning approach of students from the Confucian heritage culture problematic? Educational Research for Policy and Practice, 12(1), 5765.

U.S. General Accounting Office. (1990). Case study evaluations. Washington, D.C: Government Printing Office Retrieved from http://www.gao.gov/special.pubs/10_1_9.pdf.

Valli, L. (1997). Listening to other voices: A description of teacher reflection in the United States. Peabody Journal of Education, 72(1), 67-88.

van Manen, M. (1977). Linking ways of knowing with ways of being practical. Curriculum Inquiry, 6(3), 205-228.

Wachtel, H. K. (1998). Student evaluation of college teaching effectiveness: A brief review. Assessment \& Evaluation in Higher Education, 23(2), 191-212.

Wallace, M. J. (1991). Training foreign language teachers: A reflective approach. Cambridge: Cambridge University Press.

Wang, Q., \& Seth, N. (1998). Self-development through classroom observation: Changing perceptions in China. ELT Journal, 52(3), 205-213.

Webb, M. E. (2002). Pedagogical reasoning: Issues and solutions for the teaching and learning of ICT in secondary schools. Education and Information Technologies, 7(3), 237-255. 
Wheatley, M. (2006). Leadership and the new science: Discovering order in a chaotic world. San Francisco, CA: Berrett-Koehler.

Wichadee, S. (2012). Factors related to professional development of English language university teachers in Thailand. Journal of Education for Teaching: International Research and Pedagogy, 38(5), 615-627.

Winchester, T. M., \& Winchester, M. (2011). Exploring the impact of faculty reflection on weekly student evaluations of teaching. International Journal for Academic Development, 16(2), 119-131.

Wlodarsky, R. (2005). The professoriate: Transforming teaching practices through critical reflection and dialogue. Teaching \& Learning: The Journal of Natural Inquiry and Reflective Practice, 19(3), 156-172.

Yan, H. (2010). A brief analysis of teacher autonomy in second language acquisition. Journal of Language Teaching and Research, 1(2), 175-176.

Yao, Y., \& Grady, M. L. (2005). How do faculty make formative use of student evaluation feedback? A multiple case study. Journal of Personnel Evaluation in Education, 18(2), 107-126.

Yazan, B. (2015). Three approaches to case study methods in education: Yin, Merriam, and Stake. The Qualitative Report, 20(2), 134-152.

Yiend, J., Weller, S., \& Kinchin, I. (2014). Peer observation of teaching: The interaction between peer review and developmental models of practice. Journal of Further and Higher Education, 38(4), 465-484.

Yin, H., Lu, G., \& Wang, W. (2014). Unmasking the teaching quality of higher education: students' course experience and approaches to learning in China. Assessment \& Evaluation in Higher Education, 39(8), 949-970.

Yin, R. K. (2009). Case study research: Design and methods ( $4^{\text {th }}$ ed.). Thousand Oaks, CA: SAGE.

Yin, R. K. (2011). Qualitative research from start to finish. New York: The Guilford Press. 
Zeichner, K. M., \& Liston, D. P. (2011). Reflective teaching: An introduction. New York: Routledge. 



\section{Appendices}

\section{APPENDIX A Inventory of SET Questionnaires}

\section{INVENTORY OF STUDENT EVELUATION OF TEACHING \\ QUESTIONNAIRES}

Teachers will choose from this pool of evaluation statements on teaching effectiveness to design their own SET form with standard questions as compulsory part.

\section{Standard Questions}

Note: Rating scale

Never

(1)
Rarely

(2)
Sometimes

(3)
Usually

(4)
Always

(5)
No Opinion

(9)

\begin{tabular}{|c|l|c|c|c|c|c|c|}
\hline No. & \multicolumn{1}{|c|}{ Statements } & (1) & (2) & (3) & (4) & (5) & (9) \\
\hline 1 & $\begin{array}{l}\text { The way this teacher organised his/her teaching } \\
\text { helped me learn }\end{array}$ & $\mathrm{O}$ & $\mathrm{O}$ & $\mathrm{O}$ & $\mathrm{O}$ & $\mathrm{O}$ & $\mathrm{O}$ \\
\hline 2 & $\begin{array}{l}\text { This teacher communicated ideas and information } \\
\text { clearly }\end{array}$ & $\mathrm{O}$ & $\mathrm{O}$ & $\mathrm{O}$ & $\mathrm{O}$ & $\mathrm{O}$ & $\mathrm{O}$ \\
\hline 3 & $\begin{array}{l}\text { This teacher stimulated my interest in learning more } \\
\text { about this subject }\end{array}$ & $\mathrm{O}$ & $\mathrm{O}$ & $\mathrm{O}$ & $\mathrm{O}$ & $\mathrm{O}$ & $\mathrm{O}$ \\
\hline 4 & $\begin{array}{l}\text { This teacher treated students and their ideas with } \\
\text { respect }\end{array}$ & $\mathrm{O}$ & $\mathrm{O}$ & $\mathrm{O}$ & $\mathrm{O}$ & $\mathrm{O}$ & $\mathrm{O}$ \\
\hline
\end{tabular}

5. Overall, I would rate this teacher's effectiveness as:
(1) Very poor
(2) Poor
(3) Good
(4) Very good
(5) Excellent
(9) No Opinion

\section{Standard Questions - Open Ended}

6. Please list 2 or 3 specific things this teacher did that stimulated and/or helped you to learn.

7. Please list 2 or 3 specific things this teacher might do to improve learning.

Optional Questions - Multiple choices (choose at least 6 items in 6 categories)

$\begin{array}{cccccc}\begin{array}{l}\text { Strongly } \\ \text { disagree }\end{array} & \text { Disagree } & \begin{array}{c}\text { Neither } \\ \text { agree nor } \\ \text { disagree }\end{array} & \text { Agree } & \begin{array}{c}\text { Strongly } \\ \text { agree }\end{array} & \begin{array}{c}\text { Not } \\ \text { Applicable }\end{array} \\ \text { (1) } & \text { (2) } & \text { (3) } & \text { (4) } & \text { (5) } & \text { (9) }\end{array}$




\begin{tabular}{|c|c|c|c|c|c|c|c|}
\hline No. & Statements & (1) & (2) & (3) & (4) & (5) & (9) \\
\hline \multicolumn{8}{|c|}{ I. Personal attributes, knowledge, and preparation } \\
\hline 8 & The teacher was enthusiastic about his/her teaching & $\mathrm{O}$ & $\mathrm{O}$ & $\mathrm{O}$ & $\mathrm{O}$ & $\mathrm{O}$ & $\mathrm{O}$ \\
\hline 9 & $\begin{array}{l}\text { The teacher is dynamic and energetic in conducting the } \\
\text { lesson }\end{array}$ & $\mathrm{O}$ & $\mathrm{O}$ & $\mathrm{O}$ & $\mathrm{O}$ & $\mathrm{O}$ & $\mathrm{O}$ \\
\hline 10 & $\begin{array}{l}\text { The teacher enhances presentations with the use of } \\
\text { humour }\end{array}$ & $\mathrm{O}$ & $\mathrm{O}$ & $\mathrm{O}$ & $\mathrm{O}$ & $\mathrm{O}$ & $\mathrm{O}$ \\
\hline 11 & $\begin{array}{l}\text { The teacher's presentation style holds your interest } \\
\text { during class }\end{array}$ & $\mathrm{O}$ & $\mathrm{O}$ & $\mathrm{O}$ & $\mathrm{O}$ & $\mathrm{O}$ & $\mathrm{O}$ \\
\hline 12 & $\begin{array}{l}\text { Course materials are well prepared and carefully } \\
\text { explained }\end{array}$ & $\mathrm{O}$ & $\mathrm{O}$ & $\mathrm{O}$ & $\mathrm{O}$ & $\mathrm{O}$ & $\mathrm{O}$ \\
\hline 13 & The teaching time is used effectively & $\mathrm{O}$ & $\mathrm{O}$ & $\mathrm{O}$ & $\mathrm{O}$ & $\mathrm{O}$ & $\mathrm{O}$ \\
\hline 14 & $\begin{array}{l}\text { This teacher contrasts the implications of various } \\
\text { theories }\end{array}$ & $\mathrm{O}$ & $\mathrm{O}$ & $\mathrm{O}$ & $\mathrm{O}$ & $\mathrm{O}$ & $\mathrm{O}$ \\
\hline 15 & $\begin{array}{l}\text { This teacher presents the background or origin of } \\
\text { ideas/concepts developed in class }\end{array}$ & $\mathrm{O}$ & $\mathrm{O}$ & $\mathrm{O}$ & $\mathrm{O}$ & $\mathrm{O}$ & $\mathrm{O}$ \\
\hline 16 & $\begin{array}{l}\text { This teacher presents points of view other than his/her } \\
\text { own when appropriate }\end{array}$ & $\mathrm{O}$ & $\mathrm{O}$ & $\mathrm{O}$ & $\mathrm{O}$ & $\mathrm{O}$ & $\mathrm{O}$ \\
\hline 17 & $\begin{array}{l}\text { This teacher adequately discusses current } \\
\text { developments in field }\end{array}$ & $\mathrm{O}$ & $\mathrm{O}$ & $\mathrm{O}$ & $\mathrm{O}$ & $\mathrm{O}$ & $\mathrm{O}$ \\
\hline \multicolumn{8}{|c|}{ II. Teaching skills } \\
\hline 18 & The lesson is presented logically and systematically & $\mathrm{O}$ & $\mathrm{O}$ & $\mathrm{O}$ & $\mathrm{O}$ & $\mathrm{O}$ & $\mathrm{O}$ \\
\hline 19 & $\begin{array}{l}\text { This teacher's presentations helped me to take good } \\
\text { notes }\end{array}$ & $\mathrm{O}$ & $\mathrm{O}$ & $\mathrm{O}$ & $\mathrm{O}$ & $\mathrm{O}$ & $\mathrm{O}$ \\
\hline 20 & $\begin{array}{l}\text { Comments and feedback provided by this teacher } \\
\text { helped me learn }\end{array}$ & $\mathrm{O}$ & $\mathrm{O}$ & $\mathrm{O}$ & $\mathrm{O}$ & $\mathrm{O}$ & $\mathrm{O}$ \\
\hline 21 & $\begin{array}{l}\text { This teacher has encouraged me to think critically } \\
\text { about the subject }\end{array}$ & $\mathrm{O}$ & $\mathrm{O}$ & $\mathrm{O}$ & $\mathrm{O}$ & $\mathrm{O}$ & $\mathrm{O}$ \\
\hline 22 & $\begin{array}{l}\text { This teacher presented material in ways that held my } \\
\text { attention }\end{array}$ & $\mathrm{O}$ & $\mathrm{O}$ & $\mathrm{O}$ & $\mathrm{O}$ & $\mathrm{O}$ & $\mathrm{O}$ \\
\hline 23 & $\begin{array}{l}\text { This teacher's delivery was at a level appropriate for } \\
\text { me }\end{array}$ & $\mathrm{O}$ & $\mathrm{O}$ & $\mathrm{O}$ & $\mathrm{O}$ & $\mathrm{O}$ & $\mathrm{O}$ \\
\hline 24 & $\begin{array}{l}\text { This teacher gave constructive individual help when I } \\
\text { requested it }\end{array}$ & $\mathrm{O}$ & $\mathrm{O}$ & $\mathrm{O}$ & $\mathrm{O}$ & $\mathrm{O}$ & $\mathrm{O}$ \\
\hline 25 & $\begin{array}{l}\text { This teacher has encouraged me to think creatively } \\
\text { about this subject }\end{array}$ & $\mathrm{O}$ & $\mathrm{O}$ & $\mathrm{O}$ & $\mathrm{O}$ & $\mathrm{O}$ & $\mathrm{O}$ \\
\hline \multicolumn{8}{|c|}{ III. Content of teaching } \\
\hline 26 & $\begin{array}{l}\text { Material covered by this teacher clearly related to the } \\
\text { course objectives }\end{array}$ & $\mathrm{O}$ & $\mathrm{O}$ & $\mathrm{O}$ & $\mathrm{O}$ & $\mathrm{O}$ & $\mathrm{O}$ \\
\hline 27 & $\begin{array}{l}\text { Work set by this teacher has encouraged my study and } \\
\text { learning }\end{array}$ & $\mathrm{O}$ & $\mathrm{O}$ & $\mathrm{O}$ & $\mathrm{O}$ & $\mathrm{O}$ & $\mathrm{O}$ \\
\hline 28 & $\begin{array}{l}\text { I found this teacher's material intellectually } \\
\text { challenging }\end{array}$ & $\mathrm{O}$ & $\mathrm{O}$ & $\mathrm{O}$ & $\mathrm{O}$ & $\mathrm{O}$ & $\mathrm{O}$ \\
\hline 29 & $\begin{array}{l}\text { The majority of material this teacher presented was } \\
\text { useful to me }\end{array}$ & $\mathrm{O}$ & $\mathrm{O}$ & $\mathrm{O}$ & $\mathrm{O}$ & $\mathrm{O}$ & $\mathrm{O}$ \\
\hline \multicolumn{8}{|c|}{ IV. Student- teacher interactions } \\
\hline 30 & $\begin{array}{l}\text { This teacher friendly towards individual students, i.e. } \\
\text { achieved and maintained good rapport with me }\end{array}$ & $\mathrm{O}$ & $\mathrm{O}$ & $\mathrm{O}$ & $\mathrm{O}$ & $\mathrm{O}$ & $\mathrm{O}$ \\
\hline
\end{tabular}




\begin{tabular}{|c|c|c|c|c|c|c|c|}
\hline 31 & $\begin{array}{l}\text { This teacher encouraged my participation in class } \\
\text { discussions }\end{array}$ & $\mathrm{O}$ & $\mathrm{O}$ & $\mathrm{O}$ & $\mathrm{O}$ & $\mathrm{O}$ & $\mathrm{O}$ \\
\hline 32 & $\begin{array}{l}\text { This teacher encouraged me to raise questions in class, } \\
\text { i.e., question the instructor }\end{array}$ & $\mathrm{O}$ & $\mathrm{O}$ & $\mathrm{O}$ & $\mathrm{O}$ & $\mathrm{O}$ & $\mathrm{O}$ \\
\hline 33 & $\begin{array}{l}\text { Students are encouraged to share their ideas and } \\
\text { knowledge. }\end{array}$ & $\mathrm{O}$ & $\mathrm{O}$ & $\mathrm{O}$ & $\mathrm{O}$ & $\mathrm{O}$ & $\mathrm{O}$ \\
\hline \multicolumn{8}{|c|}{ V. Teaching aids } \\
\hline 34 & $\begin{array}{l}\text { The teacher's hand-outs have been valuable aids to my } \\
\text { learning }\end{array}$ & $\mathrm{O}$ & $\mathrm{O}$ & $\mathrm{O}$ & $\mathrm{O}$ & $\mathrm{O}$ & $\mathrm{O}$ \\
\hline 35 & $\begin{array}{l}\text { This teacher used audio-visual aids or computer } \\
\text { resources effectively to promote learning }\end{array}$ & $\mathrm{O}$ & $\mathrm{O}$ & $\mathrm{O}$ & $\mathrm{O}$ & $\mathrm{O}$ & $\mathrm{O}$ \\
\hline \multicolumn{8}{|c|}{ VI. Assignment and Evaluation } \\
\hline 36 & $\begin{array}{l}\text { Readings, homework, etc., contribute to appreciation } \\
\text { and understanding of the subject. }\end{array}$ & $\mathrm{O}$ & $\mathrm{O}$ & $\mathrm{O}$ & $\mathrm{O}$ & $\mathrm{O}$ & $\mathrm{O}$ \\
\hline 37 & $\begin{array}{l}\text { Methods of evaluating student work are fair and } \\
\text { appropriate. }\end{array}$ & $\mathrm{O}$ & $\mathrm{O}$ & $\mathrm{O}$ & $\mathrm{O}$ & $\mathrm{O}$ & $\mathrm{O}$ \\
\hline
\end{tabular}

This questionnaire was adapted from several SET questionnaires including Student

Feedback on Teaching developed by Centre for Academic Development, Victoria University of Wellington and Student Evaluation of Educational Quality developed by Marsh (1982). 


\section{APPENDIX B A Sample of Academics' TT-SET Questionnaire}

\section{STUDENT EVELUATION OF TEACHING QUESTIONNAIRES}

Academic's name: An

\section{Subject: Business English}

In order to improve teaching quality, we need your feedback on teaching performance. Could you please read the questionnaire carefully and give your ratings by blackening the appropriate cell? This questionnaire is used for research purpose. Your identity will be kept confidential.

\section{Student's General Information}

1. Gender: (1) Male (2) Female 2. Major:

3. Course:
4. Academic years:
(1)
(2)
(3)
(4)

\section{Questions}

Standard Questions

Never

(1)

\begin{abstract}
Rarely
\end{abstract}
(2)
Sometimes

(3)
Usually

(4)
Always

(5)
No Opinion

(9)

\begin{tabular}{|c|l|c|c|c|c|c|c|}
\hline No. & \multicolumn{1}{|c|}{ Statements } & (1) & (2) & (3) & (4) & (5) & (2) \\
\hline 1 & $\begin{array}{l}\text { The way this academic organised his/her teaching } \\
\text { helped me learn }\end{array}$ & $\mathrm{O}$ & $\mathrm{O}$ & $\mathrm{O}$ & $\mathrm{O}$ & $\mathrm{O}$ & $\mathrm{O}$ \\
\hline 2 & $\begin{array}{l}\text { This academic communicated ideas and information } \\
\text { clearly }\end{array}$ & $\mathrm{O}$ & $\mathrm{O}$ & $\mathrm{O}$ & $\mathrm{O}$ & $\mathrm{O}$ & $\mathrm{O}$ \\
\hline 3 & $\begin{array}{l}\text { This academic stimulated my interest in learning } \\
\text { more about this subject }\end{array}$ & $\mathrm{O}$ & $\mathrm{O}$ & $\mathrm{O}$ & $\mathrm{O}$ & $\mathrm{O}$ & $\mathrm{O}$ \\
\hline 4 & $\begin{array}{l}\text { This academic treated students and their ideas with } \\
\text { respect }\end{array}$ & $\mathrm{O}$ & $\mathrm{O}$ & $\mathrm{O}$ & $\mathrm{O}$ & $\mathrm{O}$ & $\mathrm{O}$ \\
\hline
\end{tabular}

5. Overall, I would rate this academic's effectiveness as:
(1) Very poor
(2) Poor
(3) Good
(4) Very good
(5) Excellent
(9) No Opinion

\section{Standard Questions - Open Ended}

6. Please list 2 or 3 specific things this academic did that stimulated and/or helped you to learn.

7. Please list 2 or 3 specific things this academic might do to improve learning. 
Optional Questions - Multiple choices (choose at least 6 items in 6 categories)

Strongly
disagree

(1)

\section{Disagree}

(2)
Neither

agree nor disagree

(3)
Agree

(4)
Strongly agree

(5)
Not Applicable

(9)

\begin{tabular}{|c|c|c|c|c|c|c|c|}
\hline No. & Statements & (1) & (2) & (3) & (4) & (5) & (9) \\
\hline \multicolumn{8}{|c|}{ I. Personal attributes, knowledge, and preparation } \\
\hline 8 & $\begin{array}{l}\text { The academic is dynamic and energetic in conducting } \\
\text { the lesson }\end{array}$ & $\mathrm{O}$ & $\mathrm{O}$ & $\mathrm{O}$ & $\mathrm{O}$ & $\mathrm{O}$ & $\mathrm{O}$ \\
\hline 9 & $\begin{array}{l}\text { The academic enhances presentations with the use of } \\
\text { humour }\end{array}$ & $\mathrm{O}$ & $\mathrm{O}$ & $\mathrm{O}$ & $\mathrm{O}$ & $\mathrm{O}$ & $\mathrm{O}$ \\
\hline 10 & $\begin{array}{l}\text { This academic contrasts the implications of various } \\
\text { theories }\end{array}$ & $\mathrm{O}$ & $\mathrm{O}$ & $\mathrm{O}$ & $\mathrm{O}$ & $\mathrm{O}$ & $\mathrm{O}$ \\
\hline \multicolumn{8}{|c|}{ II. Teaching skills } \\
\hline 11 & The lesson is presented logically and systematically & $\mathrm{O}$ & $\mathrm{O}$ & $\mathrm{O}$ & $\mathrm{O}$ & $\mathrm{O}$ & $\mathrm{O}$ \\
\hline 12 & feedback provided by this academic & $\mathrm{O}$ & $\mathrm{O}$ & $\mathrm{O}$ & $\mathrm{O}$ & $\mathrm{O}$ & $\mathrm{O}$ \\
\hline 13 & $\begin{array}{l}\text { has encouraged me to think critically } \\
\text { ect }\end{array}$ & $\mathrm{O}$ & $\mathrm{O}$ & $\mathrm{O}$ & $\mathrm{O}$ & $\mathrm{O}$ & 0 \\
\hline 14 & $\begin{array}{l}\text { This academic's delivery was at a level appropriate for } \\
\text { me }\end{array}$ & $\mathrm{O}$ & $\mathrm{O}$ & $\mathrm{O}$ & $\mathrm{O}$ & $\mathrm{O}$ & $\mathrm{O}$ \\
\hline 15 & $\begin{array}{l}\text { This academic has encouraged me to think creatively } \\
\text { about this subject }\end{array}$ & $\mathrm{O}$ & $\mathrm{O}$ & $\mathrm{O}$ & $\mathrm{O}$ & $\mathrm{O}$ & 0 \\
\hline \multicolumn{8}{|c|}{ III. Content of teaching } \\
\hline 16 & $\begin{array}{l}\text { Work set by this academic has encouraged my } \\
\text { and learning }\end{array}$ & $\mathrm{O}$ & $\mathrm{O}$ & $\mathrm{O}$ & $\mathrm{O}$ & $\mathrm{O}$ & $\mathrm{O}$ \\
\hline 17 & $\begin{array}{l}\text { The majority of material this academic presented was } \\
\text { useful to me }\end{array}$ & $\mathrm{O}$ & $\mathrm{O}$ & $\mathrm{O}$ & $\mathrm{O}$ & $\mathrm{O}$ & 0 \\
\hline \multicolumn{8}{|c|}{ IV. Student- academic interactions } \\
\hline 18 & $\begin{array}{l}\text { This academic friendly towards individual students, } \\
\text { i.e. achieved and maintained good rapport with me }\end{array}$ & $\mathrm{O}$ & $\mathrm{O}$ & $\mathrm{O}$ & $\mathrm{O}$ & $\mathrm{O}$ & 0 \\
\hline 19 & $\begin{array}{l}\text { This academic encouraged my participation in class } \\
\text { discussions }\end{array}$ & $\mathrm{O}$ & $\mathrm{O}$ & $\mathrm{O}$ & $\mathrm{O}$ & $\mathrm{O}$ & 0 \\
\hline 20 & $\begin{array}{l}\text { This academic encouraged me to raise questions in } \\
\text { class, i.e., question the instructor }\end{array}$ & $\mathrm{O}$ & $\mathrm{O}$ & $\mathrm{O}$ & $\mathrm{O}$ & $\mathrm{O}$ & 0 \\
\hline \multicolumn{8}{|c|}{ V. Teaching aids } \\
\hline 21 & $\begin{array}{l}\text { The academic's hand-outs have been valuable aids to } \\
\text { my learning }\end{array}$ & $\mathrm{O}$ & $\mathrm{O}$ & $\mathrm{O}$ & $\mathrm{O}$ & $\mathrm{O}$ & $\mathrm{O}$ \\
\hline \multicolumn{8}{|c|}{ VI. Assignment and Evaluation } \\
\hline 22 & $\begin{array}{l}\text { Methods of evaluating student work are fair and } \\
\text { appropriate. }\end{array}$ & $\mathrm{O}$ & $\mathrm{O}$ & $\mathrm{O}$ & $\mathrm{O}$ & $\mathrm{O}$ & 0 \\
\hline
\end{tabular}




\section{APPENDIX C Peer Observation Protocols}

\section{PRE-OBSERVATION FORM}

Peer observer and observed teacher will use this form to name areas of teaching to be observed.

Date: - Subject:- Content Area:

Teacher: - Observer:

\section{General information}

1. The area(s) in which your students felt you need to improve are:

2. The area(s) in which you feel you need to improve and need a colleague to observe is/are:

3. What is your classroom context like? Can you name the difficulties you may face?

\section{Specific information}

4. What strategies (modes of teaching, organizing, managing, and arranging) will you use? Why do you use these strategies? (or what support the things you identify?)

\section{PEER OBSERVER'S FEEDBACK FORM}

Peer observer will use this form to give feedback on teaching with the preobservation form as the basis. The peer colleagues will discuss the selection of areas that need feedback and fill in this form.

Date: Subject:

Teacher: Observer:

\section{Feedback}

\begin{tabular}{|c|c|c|}
\hline No. & Statements on areas for feedback & Notes \\
\hline 1 & & \\
\hline 2 & & \\
\hline 3 & & \\
\hline 4 & & \\
\hline$\ldots$ & & \\
\hline
\end{tabular}




\section{Suggestions for changes}

\section{TEACHER'S REFLECTION REPORT FORM}

Individual teachers will use this form to reflect on their teaching after POT by answering the following questions.

Date:

Subject:

Teacher: Observer:

\section{Reflection}

1. What have you learned about your teaching as a result of this POT process? How does your teaching approach support student learning? What has been confirmed for you? What have you become aware of that you did not know before?

2. What will you definitely keep in your teaching repertoire? Why? Focus on 1-3 aspects of your teaching.

3. What will you most likely change about your teaching approach? Why? How? Focus on $1-3$ aspects for change and outline how you intend to implement that change in your class.

4. What were especially useful and how may they lead to improvement of your classroom teaching. List any future agenda items that have emerged as a result of the POT process.

5. What other aspects are noticeable in your view? 


\section{APPENDIX D Guiding Questions for the Debriefing Session}

\section{GUIDING QUESTIONS FOR COORDINATING THE DEBRIEFING SESSION}

\section{The themes for the post-POT meeting}

The following areas will be used for coordinating the pair of participants at the debriefing session:

1) Changes the participants are going to make after peer observation of teaching

2) Premises for the changes

3) Knowledge sources for the decisions

4) Impact of experience and training on teaching practice

\section{Sample questions}

The following questions will be flexibly used to elicit for information on the themes above and stimulate the dialogue between peers.

1. What is your experience with the employment of TT-SETs and POT? Have you ever designed or tailored SET?

2. What do you think about the student feedback and how does it affect your choice of items for POT?

3. What challenges have you faced and how can you improve your teaching?

4. Why did you choose the items for POT?

5 . What changes in teaching are you going to make?

6. How are you going to make changes in teaching?

7. What makes you think that way/decide on the changes in teaching?

8. What makes a good teacher? How can he/she do to better teaching? 


\section{APPENDIX E Guiding Questions for the End-of-term Interview}

These following areas will be used as indicative for interview questions because the interview is open-ended. The questions asked will follow the way the participants respond.

1. Personal information

2. Teaching aspirations and changes

3. Pedagogy

4. Reflection and reflective practice

5. Beliefs and attitudes

6. Collegiality and collaboration

7. Usefulness of teacher-tailored SETs augmented with POT

Before the interview, the participants will be given a number of written questions that can stimulate the careful thinking about the interview issues.

\section{INTERVIEW QUESTIONS}

Thank you very much for giving me your time today. The purpose of this meeting is to hear from you about how you are aware of impact of TT-SET augmented with POT on your pedagogical reasoning. I hope you will feel free to be quite frank.

I have a list of questions (with some additional questions if necessary) I would like you to discuss in this session. Before we begin I would like you to take five minutes to look at the questions, and I will record your immediate response. I would like to collect these sheets in at the end of the session. Please remember that you should feel under no pressure to share with me or others anything with which you are not completely comfortable.

- What do you think about students and colleagues' feedback on your teaching? What change did you make in response to student feedback? Why did you change those things?

- What have you learnt about your teaching based on responding to feedback from students?

- What have you learnt about your teaching based on responding to feedback from peers?

- What have you learnt about the way the students learn and like to learn?

- How do you know about it? How do you deal with demanding students?

- Can you give some specific examples? Take listening for example.

- Why did you choose these aspects of your teaching for changes? What did you learn from attempting the changes?

- How may you do the changes in the class next term? Why?

- What have been the positive and negative aspects of self-tailored SET augmented with POT? For example, did you change your theory of teaching?

- Which steps in the POT process were most valuable? Are all the steps necessary?

- Is there a particular aspect in teaching that you are going to change? What has made you want to change that? 
- What do you think about the students' feedback on your teaching at the end of the course compared with the previous one earlier in the course?

- What is your experience with TT-SET? You have gone through the standardized SET and a tailored one? What do you think about the value of each type in your teaching?

- What did you learn about teaching when observing your peer's teaching?

- What advice would you give to a colleague employing self-tailored SET augmented with POT in the future?

- Will you continue to seek out and conduct peer observations outside this study?

- What should be done to improve POT?

- Should POT be voluntary or compulsory? Why?

- Should the observations be done by outside parties or would it be better done by peers? Please explain.

- What did cause you trouble when join in POT? What do you think are the possible challenges to those who join in POT?

- In light of this discussion what further comments would you like to make about the self-tailored SET augmented with POT?

Thank you very much for your help with my research.

Best wishes,

Phuong Nguyen 


\section{APPENDIX F Transcriber Confidentiality Agreement Form}

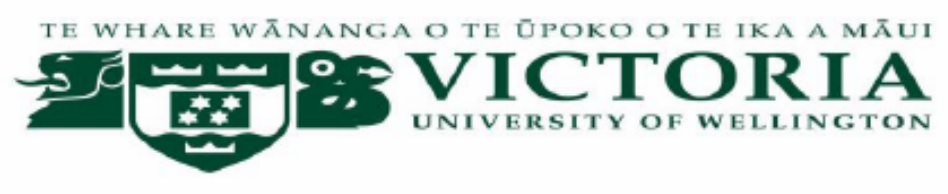

\section{Transcriber Confidentiality Agreement}

\section{Project Title:}

Case examination of teacher-tailored student evaluation of teaching augmented with peer observation of teaching in a Vietnamese university

$\mathrm{I}$, , the transcriber, agree to:

1. keep the contents of the interview material confidential by not discussing or sharing the research information in any form or format with anyone other than the researcher.

2. keep all the interview data secure while I am transcribing the interview.

3. return all the files and transcripts to the researcher when the transcribing is complete.

4. erase or destroy all research information regarding this project that is not returnable to the researcher if any (e.g. information stored on computer hard drive).

Transcriber

Name:

Signature:

Date:

Researcher

Name:

Signature:

Date: 



\section{APPENDIX G A sample extract of inductive qualitative analysis.}

\section{Example on coding data}

\begin{tabular}{|l|}
\hline From raw data through \\
themes with logical, \\
intuitive, and critical \\
thinking: \\
- Comparing and \\
contrasting, and \\
grouping the codes into \\
categories \\
- Asking why they should \\
be combined \\
Referring to the \\
definition of related \\
concepts \\
- Asking how these \\
themes were interrelated \\
and how they answered \\
the research questions \\
Asking why particular \\
themes were more \\
useful at making \\
contributions and \\
understanding \\
Making judgment based \\
on varied data, the \\
literature, and member- \\
checking \\
\end{tabular}

$$
\text { ए }
$$

\section{Premise reflection:} questioning of one's beliefs and assumptions (e.g. the teacher had a prior belief that games were not useful to student learning, but then changed her beliefs because she realised using games could make student more active and engaged in learning

\section{En: "...they could learn new words} from contexts."

Hang: "In the past, I stuck to the textbook content, but now I am more flexible and selective in teaching."
The two quotes from Dieu above were initially coded as 'process reflection and 'beliefs' respectively. however, when read the whole interview, I realised that they were related to Dieu's change in her belief about her efficacy in using varied teaching methods, i.e., premise reflection. Then, I did member checking with her and received a confirmed answer on her complete change in belief. Thus, these quotes fitted in 'premise reflection'.
Dieu: "I evaluated the effectiveness of my teaching in order to see if I should make complete changes or improve existing activities"

Dieu: 'In language teaching, if we follow 'a worn path', our
performance will appear to be boring"
$\downarrow$
A change in Dieu's belief: she meant she had been using the
same teaching methods for her class, but when she tried different
methods she realized her students were more interested.




\section{Theme: perceived impact of the intervention}

\begin{tabular}{|c|c|c|c|}
\hline Source & Quote & Code & $\begin{array}{c}\text { Category/ } \\
\text { Concept }\end{array}$ \\
\hline Hang-POF1 & $\begin{array}{l}\text { Detailed instruction should be given before carrying out activities such as discussions or exercises. It is } \\
\text { necessary to encourage weak students to communicate by forcing them to talk instead of letting them } \\
\text { volunteer for tasks. Giving some fun activities could inspire the students. The teacher should speak more } \\
\text { slowly. }\end{array}$ & $\begin{array}{l}\text { content and process } \\
\text { reflection }\end{array}$ & \multirow{9}{*}{$\begin{array}{l}\text { promoting } \\
\text { reflection }\end{array}$} \\
\hline Dieu-INT & $\begin{array}{l}\text { I evaluated the effectiveness of my teaching activities in order to see if I should make complete changes or } \\
\text { improve the existing activities }\end{array}$ & premise reflection & \\
\hline En-TRR2 & $\begin{array}{l}\text { I recognize that I need to promote the aspects which my peer highly valued and make changes to what } \\
\text { really needs improving }\end{array}$ & peer lens for learning & \\
\hline Binh-INT & Debriefing is very useful with dialogue about teaching where I could hear explanations on teaching aspects & peer lens for learning & \\
\hline Binh-TRR1 & $\begin{array}{l}\text { Collecting TT-SET helped me gain understanding of student needs whereas talking to a colleague offered } \\
\text { an opportunity to learn teaching ideas for better practice }\end{array}$ & student lens for learning & \\
\hline Dieu-INT & Writing the reflection report is a useful way to consciously evaluate teaching. & writing reflection & \\
\hline Dang-INT & Writing reflection reports is useful because it follows other steps systematically. & writing reflection & \\
\hline En-DEB & In general, SET is useful for improving teaching. & student lens for learning & \\
\hline Dang-DEB & Khoa's feedback was accurate and appropriate. & peer lens for learning & \\
\hline Dieu-INT & After getting feedback [from students and peer], I realised reflection is important for the changes & new comprehension & \multirow{4}{*}{$\begin{array}{l}\text { new } \\
\text { comprehension }\end{array}$} \\
\hline Nga-INT & $\begin{array}{l}\text { By observing the peer's teaching, I know what combination of my approaches works. Then, I became } \\
\text { more critical in analysing, for example, if these strategies work for students. I mean observations helped } \\
\text { me find evidence for my reasoning for more appropriate applications of the strategies. So, my new } \\
\text { knowledge about my teaching is that observing others makes me more critical in reflecting on teaching. }\end{array}$ & new comprehension & \\
\hline Khoa-INT & $\begin{array}{l}\text { However, after POT, I realized that if the students were excessively served that way, I would fail to impart } \\
\text { necessary knowledge }\end{array}$ & new comprehension & \\
\hline En-INT & $\begin{array}{l}\text { I often present new words with explanation of their meaning. Yet, after POT, Dieu told me to ask students } \\
\text { to find out new words in reading passages or build sentences with those words. I think it is a good idea } \\
\text { because they could learn new words from contexts. }\end{array}$ & new comprehension & \\
\hline
\end{tabular}




\begin{tabular}{|c|c|c|c|}
\hline Source & Quote & Code & $\begin{array}{c}\text { Category/ } \\
\text { Concept }\end{array}$ \\
\hline Dang-TRR2 & $\begin{array}{l}\text { I learned from Khoa, using sense of humour, video clips, and games to review lessons. Khoa gave me new } \\
\text { sources for materials, exercises, and video clips to support teaching }\end{array}$ & new comprehension & $\begin{array}{l}\text { new } \\
\text { comprehension }\end{array}$ \\
\hline Cuc-INT & $\begin{array}{l}\text { Expectation? I think my students love me because they realised how enthusiastic and passionate I am [in } \\
\text { lesson preparation and teaching] for their better learning outcomes. I believe that students may feel } \\
\text { ashamed if they do not try their best. }\end{array}$ & enhanced confidence & \multirow{5}{*}{$\begin{array}{l}\text { enhanced } \\
\text { confidence and } \\
\text { self-efficacy }\end{array}$} \\
\hline Giang-INT & $\begin{array}{l}\text { I realise that I taught more successfully this semester than before because I invested much time in lesson } \\
\text { plans with varied activities, e.g., some useful games to help students learn. }\end{array}$ & enhanced confidence & \\
\hline Dieu-INT & $\begin{array}{l}\text { I see trying making changes to teaching made practice more effective. My students became more } \\
\text { interested because they had a chance to interact with one another. Through the changes, I know them } \\
\text { more" }\end{array}$ & enhanced confidence & \\
\hline Dang-INT & ...my teaching this term could not have been good without POT... I learned a good way to review lessons & enhanced confidence & \\
\hline Dieu-INT & I believe I can manipulate impact on students with well-prepared lessons. & enhanced efficacy & \\
\hline Dang-INT & $\begin{array}{l}\text { I like TT-SET for its focus on what I consider. It shows my strengths and weaknesses for making changes. } \\
\text { S-SET provides general information that I do not know what changes should be made, informing me just } \\
\text { low or high ratings. }\end{array}$ & enhanced autonomy & \multirow{3}{*}{$\begin{array}{l}\text { enhanced } \\
\text { autonomy }\end{array}$} \\
\hline Nga-INT & $\begin{array}{l}\text { I like TT-SET because of its focus on needed information while S-SET applies for all disciplines. } \\
\text { However, using TT-SET may lead to a lack of important aspects that need considering because academics } \\
\text { have a tendency to choose items based on their strengths and students' needs. }\end{array}$ & enhanced autonomy & \\
\hline Khoa-INT & $\begin{array}{l}\text { I think the TT-SET is more valuable to me. It can help me to solve prioritized weaknesses to meet } \\
\text { students' needs, which can be achieved with help from a peer, especially an experienced one. In short, the } \\
\text { TT-SET facilitates step-by-step solution of teaching problems. }\end{array}$ & enhanced autonomy & \\
\hline Hang-VID3 & $\begin{array}{l}\text { Hang spoke more slowly in explaining new words and used an eliciting strategy to stimulate students' } \\
\text { critical thinking to find word meanings }\end{array}$ & $\begin{array}{l}\text { technical and } \\
\text { pedagogical changes }\end{array}$ & \multirow{4}{*}{$\begin{array}{l}\text { changes to } \\
\text { current } \\
\text { practice and } \\
\text { plans for future }\end{array}$} \\
\hline Binh-VID3 & $\begin{array}{l}\text { Binh sequenced her lesson more appropriately, reduced dead time (grouped students quickly for } \\
\text { discussion) }\end{array}$ & technical changes & \\
\hline Khoa-VID3 & $\begin{array}{l}\text { Khoa reduced dead time and teacher-talking time by using varied activities to engage students in learning, } \\
\text { and paid attention to individual students }\end{array}$ & $\begin{array}{l}\text { technical and } \\
\text { pedagogical changes }\end{array}$ & \\
\hline $\mathrm{Cuc}-\mathrm{INT}$ & $\begin{array}{l}\text { I changed or organised more classroom activities. I followed my students' preferred way of teaching. } \\
\text { When they wanted to be trained with listening. I gave more listening tasks... At first at the beginning of } \\
\text { the course, I did not pay attention to training integrated skills, but now I do. }\end{array}$ & pedagogical changes & \\
\hline
\end{tabular}




\begin{tabular}{|c|c|c|c|}
\hline Source & Quote & Code & $\begin{array}{c}\text { Category/ } \\
\text { Concept }\end{array}$ \\
\hline Phung-VID3 & $\begin{array}{l}\text { Phung's actions included as having more teacher-student communication by asking questions to check } \\
\text { teaching appropriateness, facilitating students' sharing ideas, giving clear instructions, and allowing more } \\
\text { student-talking time resulted from her peer's feedback }\end{array}$ & $\begin{array}{l}\text { technical and } \\
\text { pedagogical changes }\end{array}$ & \multirow{3}{*}{$\begin{array}{l}\text { changes to } \\
\text { current } \\
\text { practice and } \\
\text { plans for future }\end{array}$} \\
\hline En-INT & $\begin{array}{l}\text { I will organise more varied activities and give individual care and more suitable exercises for students at } \\
\text { different levels. I will group the good ones and those left behind for mutual support. Then I will collect } \\
\text { SET to know more about their opinions to improve teaching. }\end{array}$ & $\begin{array}{l}\text { planned changes to } \\
\text { future practice }\end{array}$ & \\
\hline Dang-INT & I will enhance students' listening skill and intonation. & $\begin{array}{l}\text { planned changes to } \\
\text { future practice }\end{array}$ & \\
\hline Dang-INT & $\begin{array}{l}\text { I trust Khoa. She is so nice. } \\
\text { I like to go on sharing teaching ideas and doing observations with Khoa }\end{array}$ & $\begin{array}{l}\text { Enhanced collaboration } \\
\text { and collegiality }\end{array}$ & \multirow{9}{*}{$\begin{array}{l}\text { enhanced } \\
\text { collaboration } \\
\text { and collegiality }\end{array}$} \\
\hline Khoa-INT & $\begin{array}{l}\text { Dang is out-going. I felt pleased to do POT with her... Dang's feedback was more helpful: more } \\
\text { constructive and more formative [i.e., more useful than TT-SET]. Because my peer knows pedagogical } \\
\text { content knowledge and the programme, she could give more precise feedback on teaching effectiveness. }\end{array}$ & enhanced collegiality & \\
\hline Dang-INT & $\begin{array}{l}\text { Khoa gave me some video-clips for teaching and showed me some useful websites where I can find useful } \\
\text { teaching materials. }\end{array}$ & enhanced collaboration & \\
\hline Dieu-INT & $\begin{array}{l}\text { POT makes learning to teach more effective and peers closer. When giving feedback, we got in harmony } \\
\text { with each other. }\end{array}$ & enhanced collegiality & \\
\hline Khoa-INT & $\begin{array}{l}\text { I want to share my teaching ideas with others and would like them to observe my teaching because this } \\
\text { collaboration with colleagues helps me reflect on teaching and gain new understanding. }\end{array}$ & enhanced collegiality & \\
\hline Phung-INT & $\begin{array}{l}\text { Our relationship is quite good. In general, we were open and truthful in giving feedback without } \\
\text { apprehension. }\end{array}$ & $\begin{array}{l}\text { enhanced collaboration } \\
\text { and collegiality }\end{array}$ & \\
\hline Giang-INT & After spending much time in conversation about teaching, I felt Phung was closer to me & $\begin{array}{l}\text { enhanced collaboration } \\
\text { and collegiality }\end{array}$ & \\
\hline Nga-INT & I thought Hang paid deference to me [at the debriefing]... Now I think our relationship is just improved. & $\begin{array}{l}\text { enhanced collaboration } \\
\text { and collegiality }\end{array}$ & \\
\hline Hang-INT & $\begin{array}{l}\text { I better understand Nga. We often shared ideas about family, life, and teaching. It is even better when I } \\
\text { observed her teaching. }\end{array}$ & $\begin{array}{l}\text { enhanced collaboration } \\
\text { and collegiality }\end{array}$ & \\
\hline
\end{tabular}




\section{APPENDIX H Review sheet for video-recordings}

\section{REVIEW SHEET FOR VIDEO-RECORDINGS OF TEACHING}

Purpose: To detect if academics implemented their planned changes after POT by reviewing videos of their teaching before, during, and after POT.

Procedure: Read the teacher's targeted aspects of teaching. As regards each feedback item, watch three videos (before, during, and after POT), record any changes the academic made in the second and third videos, and give descriptive comments.

\section{Participant:}

Part 1: Teaching aspirations and planned strategies for improvement

1. The area(s) in which students felt the participant needs to improve were:

2. The area(s) in which the participant needed to improve and needed a colleague to observe were:

$\left.{ }^{*}\right)$ List of feedback items:

\begin{tabular}{|l|c|c|}
\hline No. & Feedback items & Notes \\
\hline 1 & & \\
\hline 2 & & \\
\hline 3 & & \\
\hline
\end{tabular}

3. The strategies the participants used were:

Part 2: Learning and planned improvement after the first POT

1. Things learnt from the peer:

2. Things to keep in teaching repertoire:

3. Things to improve:

Part 3: Prompts for evaluation

\begin{tabular}{|l|c|c|}
\hline No. & Feedback items & Prompt questions \\
\hline 1 & & \\
\hline 2 & & \\
\hline 3 & & \\
\hline
\end{tabular}

Part 4: Reviewing the academic's teaching

\begin{tabular}{|l|l|l|l|l|}
\hline No. & \multicolumn{1}{|c|}{ Feedback items } & $\begin{array}{l}\text { Comments } \\
\text { on video 1 }\end{array}$ & $\begin{array}{l}\text { Comments } \\
\text { on video 2 }\end{array}$ & $\begin{array}{c}\text { Comments } \\
\text { on video 3 }\end{array}$ \\
\hline 1 & & & & \\
\hline 2 & & & & \\
\hline 3 & & & & \\
\hline$(* *)$ & Overall comments & & & \\
\hline
\end{tabular}




\section{APPENDIX I Ethics approval}

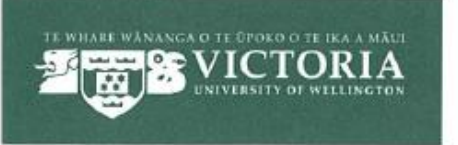

FACULTY OF EDUCATION PO Box 17-310 Wellington 6012, New Zealand Website www.victoria.ac.nz

17 December 2012

Phuong Vu Nguyen

$\mathrm{PhD}$ student

Victoria University of Wellington Faculty of Education

C/- School of Educational Psychology and Pedagogy

Donald Street

Wellington

Dear Phuong

\section{RE: Ethics application SEPP/2012/88 RM 19615}

I am pleased to advise you that your ethics application 'The role of teachertailored student feedback augmented with peer observation of teaching in quality teaching in higher education', with the required changes, has been approved by the Victoria University of Wellington Faculty of Education Ethics Committee. Please note that the approval for your research to commence is from the date of this letter.

Best wishes for your research.

Yours Sincerely

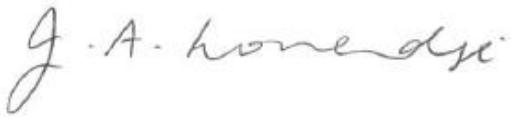

Dr Judith Loveridge

Co-Convener

Victoria University of Wellington Faculty of Education Ethics Committee 


\section{APPENDIX J Letter to the Rector of Research University}

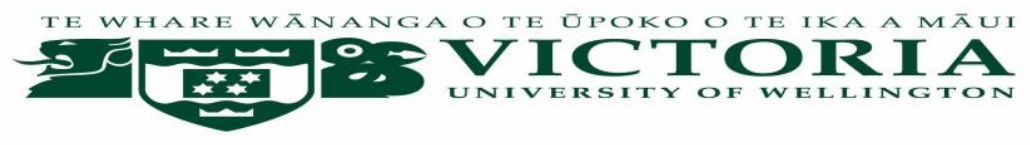

Date:

\section{Letter to the Rector of selected university}

Subject: Asking for permission to conduct research

Title of project: Case examination of teacher-tailored student evaluation of teaching augmented with peer observation of teaching in a Vietnamese university

Researcher: Phuong Vu Nguyen, Faculty of Education, School of Educational Psychology and Pedagogy, Victoria University of Wellington.

\section{Dear Sir/Madam,}

I am Phuong Vu Nguyen, a PhD student in School of Educational Psychology and Pedagogy, Victoria University of Wellington, New Zealand and lecturer at University of Economics and Law, Vietnam National University - Ho Chi Minh City. As part of this degree I am conducting research to examine the role of teacher-tailored student feedback augmented with peer observation of teaching in quality teaching in higher education in Vietnam, leading to a doctoral thesis.

The research process will be undertaken in the following manner:

- Visiting the university: Initially, I will visit your university to have a meeting with you, administrative staff, and academic staff to discuss the research.

- Selecting participants: I will invite about ten lecturers of English from the university to participate in the research. Prior to selection, all prospective participants will be provided with information about the research for their consideration. Participants will be told to ensure the confidentiality of the identity of other participants in the research. They will be invited to take part in this study with a peer colleague of their choice.

Participation will be voluntary, and each participant may withdraw at any time until data analysis commences without any disadvantage.

- Collecting data:

○ Semi-structured interviews: I would like to conduct individual interviews with up to 10 participants. Two in-depth interviews will be carried out with each participant. Each interview will last from 45 to 60 minutes. The first interview will be after they conduct teaching observation at debriefing meetings with each pair of participants; and the second one will be after they collect student feedback at the end of the course. 
- Teacher-tailored SET questionnaires: They will be used to document changes in the participants' teaching. The participants will tailor their own SET questionnaire. That means there will possibly be up to ten teacher-tailored SET forms. The forms will be delivered to their students by the participants before and after peer observation of teaching.

- Peer observation protocols: They include a pre-observation form, a peer observer's feedback form, and teacher's reflection report form.

- Video-recording: It will be used to document teachers' changes in classroom performance before POT, during the POT session, and the post-POT session. The video will be for two purposes: (i) the lecturer can review their own teaching; (ii) the researcher can do an independent analysis of the planned changes in teaching. The video camera will be focused on the teacher, not the students.

- Research journal: They will be used as a supplementary tool to record the researcher's own thoughts (feelings, reactions to experience, and reflections), reasons for decisions during interviews, debriefing sessions, and interpretations and analysis of data to reduce bias.

- Member checking: Member check through asking the participants for confirmation on the accuracy of the transcripts.

This research has been assessed and approved by Victoria University Faculty of Education Ethics Committee (No. SEPP/2012/88 RM19615). I will ensure that the identity of the participants and your university will remain confidential through the use of pseudonyms and the removal of any identifying details in the final report of the study. No information in the study will be discussed with anyone except my supervisors and the participants. All research data will be securely stored either/both in password protected files or/and in locked cupboards. All research data will be destroyed either using a pager shredder or electronically wiped within five years after the completion of the research. All video and audio recordings will be electronically destroyed. If you have questions about the way the research is being conducted you may contact the Chair of the Victoria University of Wellington Human Ethics Committee, Dr. Allison Kirkman at allison.kirkman@vuw.ac.nz.

When completed, the doctoral thesis will be submitted to the School of Educational Psychology and Pedagogy and deposited in the WJ Scott Library, and available online. It is intended that one or more articles about teacher-tailored student feedback augmented with peer observation of teaching in tertiary quality teaching in Vietnam will be submitted for publication in scholarly journals.

The participants will be required to sign a "Consent Form" to formalize their willingness to participate.

If you have any questions or would like to receive further information about the project, please feel free to contact me at phuongnv@uel.edu.vn/ Phuong.Nguyen@vuw.ac.nz. You can also contact my supervisors, Dr. Liz Jones, at 
the School of Educational Psychology and Pedagogy, Victoria University of Wellington, Room 605, Malcolm Block, Karori Campus, Karori, Wellington, Phone: +64-04 463 5939, or email: liz.jones@vuw.ac.nz, and/or Dr. Dayle Anderson, at the School of Education Policy and Implementation, Victoria University of Wellington, Room 208, Waghorn Block, Karori Campus, Wellington, Phone: + 64- 04463 9630, or email: dayle.anderson@vuw.ac.nz.

Your permission to conduct the research at your university is highly appreciated.

I will call you in the next week to organize a time to meet to discuss the research and consent form, which is attached.

Thank you very much for your support.

Yours sincerely,

Phuong Vu Nguyen 


\section{APPENDIX K Consent Form for the Rector of Research University}

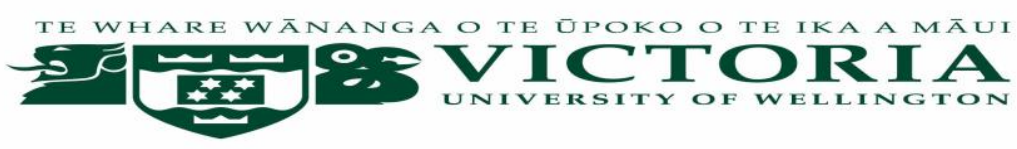

\section{RECTOR CONSENT FORM}

(to conduct the research in his/her university)

Title of project: Case examination of teacher-tailored student evaluation of teaching augmented with peer observation of teaching in a Vietnamese university

Please complete this form if you allow Phuong Vu Nguyen to conduct his doctoral research at your university.

\section{By giving his permission to conduct his study in my university I understand (please tick if you agree):}

I have been given information about this project and discussed the research project with Phuong Vu Nguyen. I have had an opportunity to ask questions and have them answered to my satisfaction.

The requirement (including being interviewed twice, video-recorded three times, and audio-recorded twice) of the participants from this university who take part in the research.

The participation in the research is voluntary and the participants may withdraw from it during the data gathering (before data collection and analysis is complete) without having to give reasons or without penalty.

$\square$ The university's identity and the participants' identity will be protected and that I may not know the identity of the participants, (or if I do I will keep their identity confidential).

All research information would be stored in password protected files and will be destroyed within five years after the completion of the research. All video and audio recordings will be electronically destroyed.

$\square$ The data collected from my university will be used for a doctoral thesis and for publication of one or more related articles in scholarly journals, and I consent for it to be used in that manner.

If I have any enquiries about the research, I can contact Phuong Vu Nguyen at phuongnv@uel.edu.vn / Phuong.Nguyen@vuw.ac.nz. I also can contact his supervisors, Dr. Liz Jones, at the School of Educational Psychology and Pedagogy, Victoria University of Wellington, Room 605, Malcolm Block, Karori Campus, Karori, Wellington 6147, Phone +64- 04463 5939, or email: liz.jones@vuw.ac.nz, and/or Dr. Dayle Anderson, at the School of Education Policy and Implementation, Victoria University of Wellington, Room 208, 
Waghorn Block, Karori Campus, Wellington, Phone: + 64- 04463 9630, or email: dayle.anderson@vuw.ac.nz.

$\square$ If I have questions about the way the research is being conducted I may contact the Chair of the Victoria University of Wellington Human Ethics Committee, Dr. Allison Kirkman at allison.kirkman@vuw.ac.nz.

By signing below,

I consent to the researcher conducting his project in this university.

$\square$ I consent to the researcher inviting lecturers in my institution to be part of the study.

Signed:

Name:

Date: 


\section{APPENDIX L Information Sheet sent to Participants of Research University}

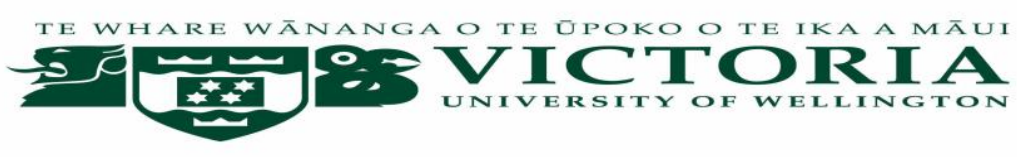

Date:

\section{INFORMATION SHEET}

\section{(Will be sent to all tertiary teachers of Business English for undergraduate programme via email)}

Case examination of teacher-tailored student evaluation of teaching augmented with peer observation of teaching in a Vietnamese university

Researcher: Phuong Vu Nguyen, Faculty of Education, School of Educational Psychology and Pedagogy, Victoria University of Wellington.

I am a PhD student in School of Educational Psychology and Pedagogy, Victoria University of Wellington and a lecturer at University of Economics and Law, Vietnam National University - Ho Chi Minh City. As part of this degree I am conducting research to examine the role of teacher-tailored student feedback augmented with peer observation of teaching in quality teaching in higher education in Vietnam.

I am inviting you to take part in this study with a peer colleague of your choice. Could you identify your peer colleague who also agrees to participate in this study? As a participant, you will tailor a student feedback form, carry out peer observation of teaching in the role of an observed teacher and peer observer. Your teaching performance will be video-recorded. The video will be used to help you review your own teaching and facilitate my independent analysis of the planned changes in teaching. You will also be interviewed about your perceptions of (i) the impact of teacher-tailored student evaluation of teaching augmented with peer observation of teaching on pedagogical reasoning; (ii) its effectiveness; and (iii) the value of student feedback via teacher-designed student evaluation of teaching compared to standardized student evaluation of teaching. Each interview will last up to one hour of your time. Prior to interview, I will give you a consent form to sign; and it is also attached to this email.

The research process will be undertaken in the following manner:

- Visiting the university: Initially, I will have visited your university to have a meeting with the Rector and administrative staff of your university to discuss the research. 
- Selecting participants: I will invite about ten lecturers of English from the university to participate in the research.

- Collecting data:

○ Semi-structured interviews: I would like to conduct individual interviews with up to 10 participants. Two in-depth interviews will be carried out with each participant. Each interview will last from 45 to 60 minutes. The first interview will be after they conduct teaching observation at debriefing meetings with each pair of participants; and the second one will be after they collect student feedback at the end of the course.

- Teacher-tailored SET questionnaires: They will be used to document changes in the participants' teaching. The participants will tailor their own SET questionnaire. That means there will possibly be up to ten teacher-tailored SET forms. The forms will be delivered to their students by the participants before and after peer observation of teaching.

- Peer observation protocols: They include a pre-observation form, a peer observer's feedback form, and teacher's reflection report form.

- Video-recording: It will be used to document teachers' changes in classroom performance before POT, during the POT session, and in the post-POT session. The camera will focus only on the teacher, not the students.

- Research journal: They will be used as a supplementary tool to record the researcher's own thoughts (feelings, reactions to experience, and reflections), reasons for decisions during interviews, debriefing sessions, and interpretations and analysis of data to reduce bias.

○ Member checking: Member check through asking the participants for confirmation on the transcripts.

You are invited to participate in this study as one of ten tertiary teachers. If you are interested in the research and want to volunteer to join my research, please reply to this email address phuongnv@uel.edu.vn or Phuong.Nguyen@ vuw.ac.nz.

I will let you know by email by (date) whether you have been selected to be part of this research. If you are selected, I will contact you and schedule the convenient time for you to be involved in the process of research.

Your participation would be voluntary, and you will be free to withdraw at any time without any disadvantage of any type and without having to explain why. Any responses that you give will be treated confidentially. Your identity will be protected through the use of a pseudonym and the removal of any identifying details.

The interview will be audio recorded for the purposes of maintaining an accurate record of your responses, and the recordings will be kept secure for a period of up to 5 years before being deleted. 
This research has been assessed and approved by Victoria University Faculty of Education Ethics Committee (No.: SEPP/2012/88 RM19615). I will ensure that the identity of the participants and your university will remain confidential through the use of pseudonyms and the removal of any identifying details in the final report of the study. No information in the study will be discussed with anyone except my supervisors and the participants. All research data will be securely stored either/both in password protected files or/and in locked cupboards. All research data will be destroyed either using a pager shredder or electronically wiped within five years after the completion of the research. All video and audio recordings will be electronically destroyed. If you have questions about the way the research is being conducted you may contact the Chair of the Victoria University of Wellington Human Ethics Committee, Dr. Allison Kirkman at allison.kirkman@vuw.ac.nz.

When completed, the doctoral thesis will be submitted to the School of Educational Psychology and Pedagogy and deposited in the WJ Scott Library, and available online. It is intended that one or more articles about teacher-tailored student feedback augmented with peer observation of teaching in tertiary quality teaching in Vietnam will be submitted for publication in scholarly journals.

If you have any questions or would like to receive further information about the project, please feel free to contact me at phuongnv@uel.edu.vn I Phuong.Nguyen@vuw.ac.nz. You can also contact my supervisors, Dr. Liz Jones, at the School of Educational Psychology and Pedagogy, Victoria University of Wellington, Room 605, Malcolm Block, Karori Campus, Karori, Wellington 6147, Phone +64- 04463 5939, or email: liz.jones@ vuw.ac.nz, and/or Dr. Dayle Anderson, at the School of Education Policy and Implementation, Victoria University of Wellington, Room 208, Waghorn Block, Karori Campus, Wellington, Phone: + 64- 04 463 9630, or email: dayle.anderson@ vuw.ac.nz. 


\section{APPENDIX M Consent Form for Participants of Research University}

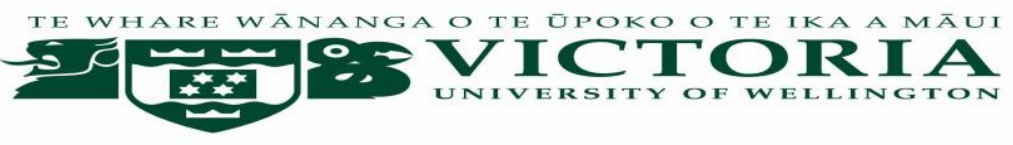

\section{PARTICIPANT CONSENT FORM}

(to be instructed in teaching observation and tailoring SET questionnaires, and to be interviewed)

Title of project: Case examination of teacher-tailored student evaluation of teaching augmented with peer observation of teaching in a Vietnamese university

Please complete this form if you would like to take part in Phuong Vu Nguyen's doctoral research.

\section{By taking part in an interview I understand that (please tick if you agree):}

I have been given information about this project and discussed the research project with Phuong Vu Nguyen. I have had an opportunity to ask questions and have them answered to my satisfaction.

I may withdraw myself (or any information I have provided) from this project (before data collection and analysis is complete) without having to give reasons or without penalty of any sort until data analysis commences.

I will be asked to:

- tailor a student feedback questionnaire from an inventory to collect feedback data on my teaching.

- join in peer observation of teaching where I will be an observed teacher and peer observer.

- participate in two semi-structured interviews. Each interview will be up to one hour. The interviews will be audio-recorded.

I agree that the researcher could video-record my teaching performance on three occasions before, during, and after peer observation of teaching for the purpose of helping me review my own teaching and facilitating the researcher's independent analysis of the planned changes in teaching.

Any information I provide will be kept confidential to the researcher, the supervisors and the person who transcribes the audio-recordings of our interviews, the published results will not use my name, and that no opinions will be attributed to me in any way that will identify me.

All data will be stored in password protected files and will be destroyed within five years after the completion of the research. All video and audio recordings will be electronically destroyed. 
I will have an opportunity to check the transcripts of the interviews and make any corrections within two weeks.

I agree that I will not disclose the identity of any other participants.

The data collected from my participation will be used for a doctoral thesis and for publication of one or more articles about teacher-tailored student feedback augmented with peer observation of teaching in tertiary quality teaching in Vietnam in scholarly journals, and I consent for it to be used in that manner. If I have any enquiries about the research, I can contact Phuong Vu Nguyen at phuongnv@uel.edu.vn / Phuong.Nguyen@vuw.ac.nz. I also can contact his supervisors, Dr. Liz Jones, at the School of Educational Psychology and Pedagogy, Victoria University of Wellington, Room 605, Malcolm Block, Karori Campus, Karori, Wellington 6147, Phone +64- 04463 5939, or email: liz.jones@vuw.ac.nz, and/or Dr. Dayle Anderson, at the School of Education Policy and Implementation, Victoria University of Wellington, Room 208, Waghorn Block, Karori Campus, Wellington, Phone: + 64- 04463 9630, or email: dayle.anderson@vuw.ac.nz.

If I have questions about the way the research is being conducted I may contact the Chair of the Victoria University of Wellington Human Ethics Committee, Dr. Allison Kirkman at allison.kirkman@vuw.ac.nz.

By signing below

I agree to participate in the research.

I would like a copy of the summary of findings of this research forwarded to me at the conclusion of the research.

or

I do not want to take part in the research at this time.

Signed:

Name:

Date: 


\section{APPENDIX N Academics' Results of TT-SET and Peer's Feedback}

The following tables on the early-term and end-of-term TT-SET results (before and after POT respectively) provide an overview on students' perceptions of each academics' teaching. The differences through paired samples t-test is significant when alpha is less than or equal to 0.05 (i.e., confidence interval is $95 \%$ ).

\section{Appendix N.1 En's results of TT-SET and peer's feedback}

Table N1.1 shows that the differences between the rating means of the early-term and end-of-term TT-SETs were not significant for En's targeted items.

Table N1.1: Difference in early-term and end-of-term TT-SETs for En

\begin{tabular}{|c|c|c|c|c|c|c|c|c|c|c|}
\hline \multirow{3}{*}{ Feedback items } & & & \multicolumn{5}{|c|}{ Paired Differences } & \multirow[b]{3}{*}{$t$} & \multirow[b]{3}{*}{ df } & \multirow{3}{*}{ 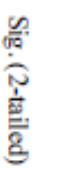 } \\
\hline & \multicolumn{2}{|c|}{ Mean } & \multirow[b]{2}{*}{ Mean } & \multirow{2}{*}{$\begin{array}{c}\text { Std. } \\
\text { Deviation }\end{array}$} & \multirow{2}{*}{$\begin{array}{l}\text { Std. } \\
\text { Error } \\
\text { Mean }\end{array}$} & \multicolumn{2}{|c|}{$\begin{array}{l}95 \% \text { Confidence } \\
\text { Interval of the } \\
\text { Difference }\end{array}$} & & & \\
\hline & SET1 & SET2 & & & & Lower & Upper & & & \\
\hline $\begin{array}{l}\text { Q1. Teaching } \\
\text { organisation }\end{array}$ & 3.6842 & 3.4737 & -.21053 & .91766 & .21053 & -.23177 & .65283 & -1.000 & 18 & .331 \\
\hline $\begin{array}{l}\text { Q2. Clarity of } \\
\text { communication }\end{array}$ & 4.0000 & 3.8500 & -.15000 & .98809 & .22094 & -.31244 & .61244 & -.679 & 19 & .505 \\
\hline Q8. Enthusiasm & 4.2500 & 4.1000 & -.15000 & .98809 & .22094 & -31244 & .61244 & -.679 & 19 & .505 \\
\hline $\begin{array}{l}\text { Q9. Delivery } \\
\text { holding interest }\end{array}$ & 3.4500 & 3.3500 & -.10000 & 1.07115 & .23952 & -40131 & .60131 & -.418 & 19 & .681 \\
\hline $\begin{array}{l}\text { Q10. Delivery at } \\
\text { appropriate } \\
\text { level }\end{array}$ & 3.9000 & 3.6000 & -.30000 & 1.08094 & .24170 & -.20589 & .80589 & -1.241 & 19 & .230 \\
\hline $\begin{array}{l}\text { Q11. Useful } \\
\text { materials }\end{array}$ & 3.8000 & 3.7500 & -.05000 & 1.27630 & .28539 & -.54733 & .64733 & -.175 & 19 & .863 \\
\hline $\begin{array}{l}\text { Q12. } \\
\text { Encouragement } \\
\text { of discussions }\end{array}$ & 4.3000 & 4.0500 & -.25000 & .85070 & .19022 & -.14814 & .64814 & -1.314 & 19 & .204 \\
\hline $\begin{array}{l}\text { Q14. Effective } \\
\text { use of visual } \\
\text { aids and } \\
\text { computer } \\
\text { resources }\end{array}$ & 4.0500 & 4.2000 & .15000 & .81273 & .18173 & -.53037 & .23037 & .825 & 19 & .419 \\
\hline $\begin{array}{l}\text { Q16. } \\
\text { Appropriateness } \\
\text { of learning } \\
\text { evaluation }\end{array}$ & 4.1000 & 4.0500 & -.05000 & 82558 & .18460 & -.33638 & .43638 & -.271 & 19 & .789 \\
\hline
\end{tabular}




\begin{tabular}{|c|c|}
\hline SET1 & SET2 \\
\hline \multicolumn{2}{|c|}{ 6. Please list 2 or 3 specific things this teacher did that stimulated and/or helped you to learn. } \\
\hline $\begin{array}{l}\text { Teaching vocabulary well (3 ideas), developing } \\
\text { speaking skills (2), regular revision (4), giving } \\
\text { clear instructions (3), organising fun activities } \\
\text { such as listening to songs, storytelling, and } \\
\text { useful games (30), showing rapport (3), } \\
\text { group/pair-work (10), giving bonus grades (16) }\end{array}$ & $\begin{array}{l}\text { Teaching vocabulary well (4 ideas), organising } \\
\text { fun activities such as listening to songs, } \\
\text { storytelling, and useful games (22), group/pair- } \\
\text { work (3), giving bonus grades (9), clear } \\
\text { presentations with practical examples (3), } \\
\text { encouraging students to give ideas and } \\
\text { respecting students' ideas (2), giving exercises } \\
\text { (1), and checking attendance (2) }\end{array}$ \\
\hline \multicolumn{2}{|c|}{ 7. Please list 2 or 3 specific things this teacher might do to improve learning. } \\
\hline $\begin{array}{l}\text { Correcting pronunciation (3), using translation } \\
\text { (5), using useful games (3), instructing how to } \\
\text { use new words learnt (4), using content } \\
\text { appropriate for students (6), developing } \\
\text { speaking and listening skills (7), consolidating } \\
\text { what was learnt (8) }\end{array}$ & $\begin{array}{l}\text { Teaching more business English with } \\
\text { appropriate materials (2), giving more } \\
\text { practical examples (1), giving sample tests and } \\
\text { exercises (3), organising entertaining activities } \\
\text { such as song and games (5), group-work (1), } \\
\text { training pronunciation and speaking, listening, } \\
\text { reading, and writing skills (7), organising } \\
\text { competition and giving bonus grades (3) }\end{array}$ \\
\hline
\end{tabular}

Peer feedback after POT1 and POT2 on En's practice was about the same for teaching organisation, communication of ideas, enthusiasm, interest maintenance, and appropriateness, as shown in Table N1.2.

Table N1.2: En's peer feedback

\begin{tabular}{|c|c|c|}
\hline & POTl feedback & POT2 feedback \\
\hline $\begin{array}{l}\text { Good } \\
\text { aspects }\end{array}$ & $\begin{array}{l}\text { - Properly arranged activities with } \\
\text { appropriate timing } \\
\text { - sing games were used to stimulate } \\
\text { learners' interest } \\
\text { - Presenting the lesson logically thanks } \\
\text { to good preparation with wide } \\
\text { knowledge } \\
\text { - Meeting the course objectives } \\
\text { - Being suitable for students' level } \\
\text { - The academic being enthusiastic, } \\
\text { dynamic, and formal } \\
\text { - Having good pronunciation, and class } \\
\text { management }\end{array}$ & $\begin{array}{l}\text { - Properly arranged activities for } \\
\text { teaching delivery with all necessary } \\
\text { steps (warming-up, pre-reading, } \\
\text { while-reading, and post-reading } \\
\text { activities). } \\
\text { - Being well prepared and having } \\
\text { knowledge of the topic. Clear, easy to } \\
\text { follow, and formal teaching } \\
\text { - Good teaching skills with combination } \\
\text { of varied methods such as } \\
\text { communicative language teaching and } \\
\text { grammar translation to promote } \\
\text { students' understanding of knowledge }\end{array}$ \\
\hline $\begin{array}{l}\text { Aspects for } \\
\text { attention }\end{array}$ & - Teaching vocabulary in isolation & - Examples for clarity of lesson \\
\hline Suggestions & $\begin{array}{l}\text { - Necessary to present vocabulary in } \\
\text { context } \\
\text { - Should ask students about } \\
\text { vocabulary's position, word class, and } \\
\text { meaning; and asking them to build } \\
\text { sentences with the new words } \\
\text { - Should call many pairs so that the } \\
\text { whole class could have a chance to } \\
\text { participate and give bonuses to } \\
\text { encourage learning to encourage their } \\
\text { engagement }\end{array}$ & $\begin{array}{l}\text { - Necessary to give some practical } \\
\text { examples } \\
\text { - Should design some activities for pair- } \\
\text { work or group-work to facilitate class } \\
\text { interactions and build up interesting } \\
\text { atmosphere }\end{array}$ \\
\hline
\end{tabular}




\section{Appendix N.2 Dang's results of TT-SET and peer's feedback}

Table N2.1 shows that the differences between the rating means of the early-term and end-of-term TT-SETs were not significant for Dang's targeted items. In general, the ratings were high.

Table N2.1: Difference in early-term and end-of-term TT-SETs for Dang

\begin{tabular}{|c|c|c|c|c|c|c|c|c|c|c|}
\hline \multirow{3}{*}{ Feedback items } & & & \multicolumn{5}{|c|}{ Paired Differences } & \multirow[b]{3}{*}{$t$} & \multirow[b]{3}{*}{ df } & \multirow{3}{*}{ 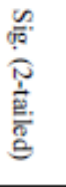 } \\
\hline & \multicolumn{2}{|c|}{ Mean } & \multirow[b]{2}{*}{ Mean } & \multirow{2}{*}{$\begin{array}{c}\text { Std. } \\
\text { Deviation }\end{array}$} & \multirow{2}{*}{$\begin{array}{l}\text { Std. } \\
\text { Error } \\
\text { Mean }\end{array}$} & \multicolumn{2}{|c|}{$\begin{array}{l}95 \% \text { Confidence } \\
\text { Interval of the } \\
\text { Difference }\end{array}$} & & & \\
\hline & SET1 & SET2 & & & & Lower & Upper & & & \\
\hline $\begin{array}{l}\text { Q1. Teaching } \\
\text { organisation }\end{array}$ & 3.8000 & 3.9200 & .12000 & .97125 & .19425 & -28091 & .52091 & .618 & 24 & .543 \\
\hline $\begin{array}{l}\text { Q2. Clarity of } \\
\text { communication }\end{array}$ & 4.3103 & 4.2069 & -.10345 & .85960 & .15962 & -.43042 & .22353 & -.648 & 28 & 522 \\
\hline $\begin{array}{l}\text { Q8. Dynamism } \\
\text { and being } \\
\text { energetic }\end{array}$ & 4.2903 & 4.3548 & .06452 & .89202 & .16021 & -.26268 & .39171 & .403 & 30 & .690 \\
\hline $\begin{array}{l}\text { Q13. Delivery at } \\
\text { appropriate } \\
\text { level }\end{array}$ & 3.6786 & 4.0357 & .35714 & 1.22366 & .23125 & -.11734 & .83163 & 1.544 & 27 & .134 \\
\hline $\begin{array}{l}\text { Q14. } \\
\text { Constructive } \\
\text { individual } \\
\text { support }\end{array}$ & 4.0333 & 4.2000 & .16667 & .83391 & .15225 & -.14472 & .47805 & 1.095 & 29 & .283 \\
\hline Q18. Rapport & 4.3448 & 4.6207 & .27586 & .79716 & .14803 & -.02736 & .57909 & 1.864 & 28 & .073 \\
\hline $\begin{array}{l}\text { Q19. } \\
\text { Encouragement } \\
\text { of discussions }\end{array}$ & 4.4828 & 4.5172 & .03448 & .77840 & .14455 & -.26161 & .33057 & .239 & 28 & .813 \\
\hline $\begin{array}{l}\text { Q21. } \\
\text { Encouragement } \\
\text { of ideas and } \\
\text { knowledge } \\
\text { sharing }\end{array}$ & 4.3043 & 4.3913 & .08696 & .84816 & .17685 & -.27981 & .45373 & .492 & 22 & .628 \\
\hline $\begin{array}{l}\text { Q23. Effective } \\
\text { use of visual } \\
\text { aids and } \\
\text { computer } \\
\text { resources }\end{array}$ & 4.0370 & 4.2963 & .25926 & .81300 & .15646 & -.06235 & .58087 & 1.657 & 26 & .110 \\
\hline $\begin{array}{l}\text { Q25. } \\
\text { Appropriateness } \\
\text { of learning } \\
\text { evaluation }\end{array}$ & 4.0000 & 4.2308 & .23077 & .76460 & .14995 & -.07806 & .53960 & 1.539 & 25 & .136 \\
\hline
\end{tabular}




\begin{tabular}{|c|c|}
\hline SET1 & SET2 \\
\hline \multicolumn{2}{|c|}{ 6. Please list 2 or 3 specific things this teacher did that stimulated and/or helped you to learn. } \\
\hline $\begin{array}{l}\text { Encouraging self-study (2 ideas), using } \\
\text { interesting visual aids, games with fun and other } \\
\text { entertaining activities (17) clear presentations of } \\
\text { lessons with clear promunciation and modern } \\
\text { methods (7), friendly and enthusiastic teacher } \\
\text { (11), listening to and sharing with students (4) } \\
\text { checking lesson preparation and vocabulary (3), } \\
\text { group/pair-work (8), discussion (7)giving bomus } \\
\text { grades (11) }\end{array}$ & $\begin{array}{l}\text { Organising interesting activities such as } \\
\text { storytelling, games, English songs, video clips } \\
\text { (34 ideas), showing rapport (3), appreciating } \\
\text { students' effort (1), giving exercises (1), giving } \\
\text { bomus grades (9), enthusiasm (1), organising } \\
\text { group-work (7), guiding learning methods (1) }\end{array}$ \\
\hline \multicolumn{2}{|c|}{ 7. Please list 2 or 3 specific things this teacher might do to improve learning. } \\
\hline $\begin{array}{l}\text { Using more visual aids (1), listening to students' } \\
\text { ideas (2), providing more appropriate materials } \\
\text { (9), guiding learning methods and teaching } \\
\text { more vocabulary (6), training more speaking } \\
\text { skills (6), providing appropriate topics for } \\
\text { discussion (1), organising group-work for } \\
\text { discussion (10), encouraging lesson preparation } \\
\text { by giving bomus grades (2), playing English } \\
\text { songs (4) }\end{array}$ & $\begin{array}{l}\text { Providing more materials for developing } \\
\text { language skills (14), guiding learning methods } \\
\text { (3), organising varied activities (3), being } \\
\text { friendlier (2), listing to students' feedback to } \\
\text { deliver lessons at an appropriate level (1), } \\
\text { revision and giving sample tests (3), paying } \\
\text { care to individual students (1) }\end{array}$ \\
\hline
\end{tabular}

Dang received similar peer feedback and suggestions after POT1 and POT2 on communication of ideas, technical aids, teaching organisation, interest maintenance, and appropriateness, as shown in Table N2.2.

Table N2.2: Dang's peer feedback

\begin{tabular}{|c|c|c|}
\hline & POT 1 feedback & POT2 feedback \\
\hline $\begin{array}{l}\text { Good } \\
\text { aspects }\end{array}$ & $\begin{array}{l}\text { - Teaching delivery at a level } \\
\text { appropriate for the students } \\
\text { - Effective use of PowerPoint } \\
\text { - Good review of lessons } \\
\text { - Creatively and interestingly organised } \\
\text { communication activities were to } \\
\text { facilitate practice } \\
\text { - Integrated real situations } \\
\text { - Good class management }\end{array}$ & $\begin{array}{l}\text { - Giving clear explanations with simple } \\
\text { vocabulary appropriate for students' } \\
\text { level } \\
\text { - Using Power point for delivery to } \\
\text { engage students in learning } \\
\text { - Using group presentations to check } \\
\text { learning and preparation } \\
\text { - Using games to review lessons and } \\
\text { stimulate interest }\end{array}$ \\
\hline $\begin{array}{l}\text { Aspects for } \\
\text { attention }\end{array}$ & $\begin{array}{l}\text { - Timing } \\
\text { - Contexts for language use } \\
\text { - Fun activities } \\
\text { - Intonation } \\
\end{array}$ & $\begin{array}{l}\text { - Feedback } \\
\text { - Fun activities } \\
\text { - Intonation }\end{array}$ \\
\hline Suggestions & $\begin{array}{l}\text { Necessary to: } \\
\text { - balance time for the sections } \\
\text { - provide specific contexts so that } \\
\text { students could give good examples } \\
\text { - ask students to reproduce 'requests' in } \\
\text { drama } \\
\text { Importance of using intonation to } \\
\text { attract attention }\end{array}$ & $\begin{array}{l}\text { - Should give feedback after group } \\
\text { presentations } \\
\text { - Using more fun activities } \\
\text { - Should use intonation to attract } \\
\text { attention }\end{array}$ \\
\hline
\end{tabular}




\section{Appendix N.3 Dieu's results of TT-SET and peer's feedback}

Table N3.1 shows that there were not significant differences in the rating means of the early-term and end-of-term TT-SETs for most of selected items except for one item being rated lower.

Table N3.1: Difference in early-term and end-of-term TT-SETs for Dieu

\begin{tabular}{|c|c|c|c|c|c|c|c|c|c|c|}
\hline \multirow{3}{*}{ Feedback items } & & & \multicolumn{5}{|c|}{ Paired Differences } & \multirow[b]{3}{*}{$\mathrm{t}$} & \multirow[b]{3}{*}{ df } & \multirow{3}{*}{ 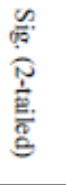 } \\
\hline & \multicolumn{2}{|c|}{ Mean } & \multirow[b]{2}{*}{ Mean } & \multirow{2}{*}{$\begin{array}{c}\text { Std. } \\
\text { Deviation }\end{array}$} & \multirow{2}{*}{$\begin{array}{l}\text { Std. } \\
\text { Error } \\
\text { Mean }\end{array}$} & \multicolumn{2}{|c|}{$\begin{array}{l}95 \% \text { Confidence } \\
\text { Interval of the } \\
\text { Difference }\end{array}$} & & & \\
\hline & SET1 & SET2 & & & & Lower & Upper & & & \\
\hline $\begin{array}{l}\text { Q1. Teaching } \\
\text { organisation }\end{array}$ & 3.5455 & 3.6364 & .09091 & .94388 & .28459 & -.72502 & .54320 & .319 & 10 & .756 \\
\hline $\begin{array}{l}\text { Q3. Stimulation } \\
\text { of learning } \\
\text { interest }\end{array}$ & 3.7500 & 3.5000 & -25000 & .86603 & .25000 & -.30025 & .80025 & -1.000 & 11 & .339 \\
\hline Q12. Feedback & 3.7143 & 3.7857 & .07143 & .82874 & .22149 & -.54993 & .40707 & .322 & 13 & .752 \\
\hline $\begin{array}{l}\text { Q13. } \\
\text { Encouragement } \\
\text { of critical } \\
\text { thinking }\end{array}$ & 3.5385 & 3.6923 & .15385 & .89872 & .24926 & -.69694 & .38924 & .617 & 12 & .549 \\
\hline $\begin{array}{l}\text { Q15. } \\
\text { Encouragement } \\
\text { of creative } \\
\text { thinking }\end{array}$ & 3.5714 & 3.6429 & .07143 & 1.20667 & .32250 & -.76814 & .62528 & .221 & 13 & .828 \\
\hline $\begin{array}{l}18 . \\
\text { Encouragement } \\
\text { of discussions }\end{array}$ & 4.6154 & 4.0769 & -.53846 & .77625 & .21529 & .06938 & 1.00754 & -2.501 & 12 & .028 \\
\hline $\begin{array}{l}\text { Q19. } \\
\text { Encouragement } \\
\text { of ideas and } \\
\text { knowledge } \\
\text { sharing }\end{array}$ & 4.5000 & 4.0714 & -.42857 & .93761 & .25059 & -.11279 & .96993 & -1.710 & 13 & .111 \\
\hline
\end{tabular}

\begin{tabular}{|c|c|}
\hline SET1 & SET2 \\
\hline \multicolumn{2}{|c|}{ 6. Please list 2 or 3 specific things this teacher did that stimulated and/or helped you to learn. } \\
\hline $\begin{array}{l}\text { Organising group/pair-work (9), giving bonus } \\
\text { grades (13), organising interesting activities } \\
\text { such as games, storytelling, and English songs } \\
\text { (5), pronunciation correction (1), introducing } \\
\text { good materials (2) }\end{array}$ & $\begin{array}{l}\text { Organising interesting activities such as games, } \\
\text { roleplay, dramas, English songs ( } 25 \text { ideas), } \\
\text { developing language skills (1), providing more } \\
\text { materials (2), giving quizzes (1), giving bonus } \\
\text { grades (13) }\end{array}$ \\
\hline \multicolumn{2}{|c|}{ 7. Please list 2 or 3 specific things this teacher might do to improve learning. } \\
\hline $\begin{array}{l}\text { Developing writing and speaking skills with } \\
\text { pronunciation guides (9), teaching more } \\
\text { vocabulary (5), activities supportive of learning } \\
\text { such as quizzes, games, English movies (2), } \\
\text { providing more materials and exercises (3), } \\
\text { more group-work discussion (2), guiding } \\
\text { learning methods (2), exchange with guest } \\
\text { speakers (1) }\end{array}$ & $\begin{array}{l}\text { Providing more homework and materials (9), } \\
\text { organising group-work (4), being less strict by } \\
\text { giving easier tests and higher grades (3), giving } \\
\text { feedback and encouragement (1), developing } \\
\text { language skills (3), exchange with other classes } \\
\text { (2), allowing long break-time (1), giving } \\
\text { clearer instruction (3), games (1), sample tests } \\
\text { (3), flexible schedule for the course (1) }\end{array}$ \\
\hline
\end{tabular}

Dieu's peer identified the same good aspects on her teaching in POT2 as in POT1. Suggestions were given on attention to low-achieving learners and a strategy for presenting vocabulary, as shown in Table N3.1. 
Table N3.2: Dieu's peer feedback

\begin{tabular}{|c|c|c|}
\hline & POT 1 feedback & POT2 feedback \\
\hline $\begin{array}{l}\text { Good } \\
\text { aspects }\end{array}$ & $\begin{array}{l}\text { - Properly- organised activities with } \\
\text { appropriate timing } \\
\text { - Varied activities leading to } \\
\text { - stimulating students' interest and } \\
\text { focus } \\
\text { - Clear and logical presentations with } \\
\text { pictures and guiding questions to } \\
\text { encourage the students to think } \\
\text { critically about the subject } \\
\text { - Organising group-work and giving } \\
\text { bonuses that encouraged students' } \\
\text { effort } \\
\text { - Showing enthusiasm and formality in } \\
\text { teaching } \\
\text { - Suitable content knowledge delivery } \\
\text { to the students' level }\end{array}$ & $\begin{array}{l}\text { - Properly- organised activities with } \\
\text { appropriate timing } \\
\text { - Giving bonuses that motivated and } \\
\text { interested students a lot } \\
\text { - Clear presentations with detailed steps } \\
\text { - Creating competition among groups, } \\
\text { forcing students to think critically } \\
\text { about the task } \\
\text { - Being dignified in clear speech, } \\
\text { enthusiastic, and well-prepared }\end{array}$ \\
\hline $\begin{array}{l}\text { Aspects for } \\
\text { attention }\end{array}$ & $\begin{array}{l}\text { - Attention to weak/low-achieving } \\
\text { students }\end{array}$ & - No opinion \\
\hline Suggestions & $\begin{array}{l}\text { - Should provide more words from the } \\
\text { content of the listening passage to } \\
\text { help weak students catch up with the } \\
\text { task }\end{array}$ & - No opinion \\
\hline
\end{tabular}

\section{Appendix N.4 Hang's results of TT-SET and peer's feedback}

Table N4.1 shows that the differences between the rating means of early-term and endof-term SETs were not significant for most items except for only one item being rated higher. 
Table N4.1: Difference in early-term and end-of-term TT-SETs for Hang

\begin{tabular}{|c|c|c|c|c|c|c|c|c|c|c|}
\hline \multirow[b]{3}{*}{ Feedback items } & \multirow{2}{*}{\multicolumn{2}{|c|}{ Mean }} & \multicolumn{5}{|c|}{ Paired Differences } & \multirow[b]{3}{*}{$\mathrm{t}$} & \multirow[b]{3}{*}{ df } & \multirow{3}{*}{ 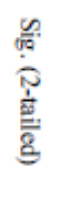 } \\
\hline & & & \multirow[b]{2}{*}{ Mean } & \multirow{2}{*}{$\begin{array}{c}\text { Std. } \\
\text { Deviation }\end{array}$} & \multirow{2}{*}{$\begin{array}{l}\text { Std. } \\
\text { Error } \\
\text { Mean }\end{array}$} & \multicolumn{2}{|c|}{$\begin{array}{l}95 \% \text { Confidence } \\
\text { Interval of the } \\
\text { Difference }\end{array}$} & & & \\
\hline & SET1 & SET2 & & & & Lower & Upper & & & \\
\hline $\begin{array}{l}\text { Q1. Teaching } \\
\text { organisation }\end{array}$ & 4.1538 & 4.5385 & .38462 & .96077 & .26647 & -19597 & .96520 & 1.443 & 12 & .175 \\
\hline $\begin{array}{l}\text { Q3. Stimulation } \\
\text { of interest in } \\
\text { learning }\end{array}$ & 4.0769 & 4.8462 & .76923 & 92681 & .25705 & .20917 & 1.32930 & 2.993 & 12 & .011 \\
\hline $\begin{array}{l}\text { Q11. Delivery } \\
\text { holding interest }\end{array}$ & 3.7500 & 4.1667 & .41667 & .79296 & .22891 & -.08716 & .92049 & 1.820 & 11 & .096 \\
\hline $\begin{array}{l}\text { Q13. Effective } \\
\text { use of time }\end{array}$ & 4.0833 & 4.5000 & .41667 & .66856 & .19300 & -.00811 & .84145 & 2.159 & 11 & .054 \\
\hline $\begin{array}{l}\text { Q24. Work set } \\
\text { encouraging } \\
\text { learning }\end{array}$ & 3.9167 & 4.1667 & .25000 & .75378 & .21760 & -.22893 & .72893 & 1.149 & 11 & .275 \\
\hline $\begin{array}{l}\text { Q27. } \\
\text { Encouragement } \\
\text { of discussions }\end{array}$ & 4.2500 & 4.7500 & .50000 & .90453 & .26112 & -.07471 & 1.07471 & 1.915 & 11 & .082 \\
\hline $\begin{array}{l}\text { Q28. } \\
\text { Encouragement } \\
\text { of questioning }\end{array}$ & 4.1818 & 4.6364 & .45455 & .68755 & .20730 & -.00736 & .91645 & 2.193 & 10 & .053 \\
\hline $\begin{array}{l}\text { Q29. } \\
\text { Encouragement } \\
\text { of idea and } \\
\text { knowledge } \\
\text { sharing }\end{array}$ & 4.2727 & 4.6364 & .36364 & .80904 & .24393 & -.17988 & .90716 & 1.491 & 10 & .167 \\
\hline
\end{tabular}

\begin{tabular}{|c|c|}
\hline SET1 & SET2 \\
\hline \multicolumn{2}{|c|}{ 6. Please list 2 or 3 specific things this teacher did that stimulated and/or helped you to learn. } \\
\hline $\begin{array}{l}\text { Organising competitive games and roleplay } \\
\text { (12), giving bomus grades (1), encouraging } \\
\text { students to give opinions (1), friendly teacher } \\
\text { (3), organising group-work (2), interesting } \\
\text { methods (1) }\end{array}$ & $\begin{array}{l}\text { Organising competitive games, dramas, } \\
\text { English songs, roleplay, and other interesting } \\
\text { activities (20), providing basic knowledge (2), } \\
\text { friendly teacher (1), using more English (2), } \\
\text { giving feedback to students (1) }\end{array}$ \\
\hline \multicolumn{2}{|c|}{ 7. Please list 2 or 3 specific things this teacher might do to improve learning. } \\
\hline $\begin{array}{l}\text { Encouraging communication in English such as } \\
\text { discussion or presentations (5), developing } \\
\text { listening and speaking skills (3), providing more } \\
\text { materials (7) }\end{array}$ & $\begin{array}{l}\text { Developing listening, speaking, reading, } \\
\text { writing skills (7), giving more practice in u. } \\
\text { English (4), extra-curricular activities (1), } \\
\text { using the previous teaching methods (1), } \\
\text { providing basic knowledge and materials ( }\end{array}$ \\
\hline
\end{tabular}

Peer feedback on Hang's targeted aspects of teaching was mostly positive, including real-life examples to engage students in learning, group/pair-work activities, and rapport. Yet, suggestions were about management of discussions, instruction, and speech flow, developing language skills, and engaging students (see Table N4.2). 
Table N4.2: Hang's peer feedback

\begin{tabular}{|c|c|c|}
\hline & POT 1 feedback & POT2 feedback \\
\hline $\begin{array}{l}\text { Good } \\
\text { aspects }\end{array}$ & $\begin{array}{l}\text { - Providing enriched information with } \\
\text { real-life examples which stimulated } \\
\text { interest in learning } \\
\text { - Organising pair-work and group-work } \\
\text { for students to show opinions with } \\
\text { academic's support and correction } \\
\text { - Moving around to give help }\end{array}$ & $\begin{array}{l}\text { - Clear lead-in instructions that helped } \\
\text { students guess what they were going } \\
\text { to listen to } \\
\text { - Clearly explaining tricky expressions } \\
\text { and sentences by linking them to } \\
\text { real-life situations } \\
\text { - Encouraging students to talk about } \\
\text { their real-life aspirations }\end{array}$ \\
\hline $\begin{array}{l}\text { Aspects for } \\
\text { attention }\end{array}$ & $\begin{array}{l}\text { - Group management: Dominant vs. } \\
\text { quiet students } \\
\text { - Not clear and detailed instructions } \\
\text { - Fast speech flow }\end{array}$ & $\begin{array}{l}\text { - Lack focus on skills and facilitation of } \\
\text { students engagement in exploring their } \\
\text { understanding of lessons. }\end{array}$ \\
\hline Suggestions & $\begin{array}{l}\text { - Should give detailed instruction } \\
\text { before carrying out activities such as } \\
\text { discussions or exercises } \\
\text { - Should encourage weak students to } \\
\text { communicate by forcing them to talk } \\
\text { instead of letting them volunteer for } \\
\text { tasks } \\
\text { - Should use some fun activities to } \\
\text { inspire students } \\
\text { - Should speak more slowly }\end{array}$ & $\begin{array}{l}\text { - Necessary to introduce or review } \\
\text { skills applicable to the task for the } \\
\text { listening activity and some difficult } \\
\text { unfamiliar expressions in the passages } \\
\text { - For the reading activity, should } \\
\text { encourage students to interpret the } \\
\text { meanings of tricky expressions } \\
\text { instead of giving explanations to see } \\
\text { how they understand the reading } \\
\text { passage }\end{array}$ \\
\hline
\end{tabular}

\section{Appendix N.5 Khoa's results of TT-SET and peer's feedback}

Table N5.1 shows that there were no significant differences on rating means for Khoa's all selected items.

Table N5.1: Difference in early-term and end-of-term TT-SETs for Khoa

\begin{tabular}{|c|c|c|c|c|c|c|c|c|c|c|}
\hline \multirow{3}{*}{ Feedback items } & \multirow{2}{*}{\multicolumn{2}{|c|}{ Mean }} & \multicolumn{5}{|c|}{ Paired Differences } & \multirow[b]{3}{*}{$t$} & \multirow[b]{3}{*}{ df } & \multirow{3}{*}{ 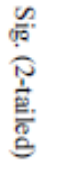 } \\
\hline & & & \multirow[b]{2}{*}{ Mean } & \multirow{2}{*}{$\begin{array}{c}\text { Std. } \\
\text { Deviation }\end{array}$} & \multirow{2}{*}{$\begin{array}{l}\text { Std. } \\
\text { Error } \\
\text { Mean }\end{array}$} & \multicolumn{2}{|c|}{$\begin{array}{l}95 \% \text { Confidence } \\
\text { Interval of the } \\
\text { Difference }\end{array}$} & & & \\
\hline & SET1 & SET2 & & & & Lower & Upper & & & \\
\hline $\begin{array}{l}\text { Q1. Teaching } \\
\text { organisation }\end{array}$ & 3.8667 & 4.0000 & .13333 & .83381 & .21529 & -32841 & .59508 & .619 & 14 & .546 \\
\hline $\begin{array}{l}\text { Q3. Stimulation } \\
\text { of interest in } \\
\text { learning }\end{array}$ & 3.9412 & 3.7059 & -.23529 & 1.43742 & .34863 & -97435 & .50376 & -675 & 16 & .509 \\
\hline $\begin{array}{l}\text { Q10. Use of } \\
\text { humour }\end{array}$ & 4.5294 & 4.1765 & -.35294 & 1.41161 & .34237 & -1.07872 & .37284 & -1.031 & 16 & .318 \\
\hline $\begin{array}{l}\text { Q12. Logical } \\
\text { and systematic } \\
\text { presentation }\end{array}$ & 4.0000 & 3.9412 & -.05882 & .96635 & .23437 & -.55567 & .43803 & -251 & 16 & .805 \\
\hline $\begin{array}{l}\text { Q15. Useful } \\
\text { materials }\end{array}$ & 4.2500 & 3.7500 & -.50000 & 1.15470 & .28868 & -1.11530 & .11530 & -1.732 & 15 & .104 \\
\hline Q16. Rapport & 4.1176 & 3.8824 & -.23529 & 1.56243 & .37894 & -1.03862 & .56803 & -.621 & 16 & .543 \\
\hline $\begin{array}{l}\text { Q17. } \\
\text { Encouragement } \\
\text { of idea and } \\
\text { knowledge } \\
\text { sharing }\end{array}$ & 4.2000 & 3.6000 & -.60000 & 1.29835 & .33523 & -1.31900 & .11900 & -1.790 & 14 & .095 \\
\hline $\begin{array}{l}\text { Q19. } \\
\text { Appropriateness } \\
\text { of learning } \\
\text { evaluation }\end{array}$ & 3.9412 & 4.0588 & .11765 & 1.16632 & .28287 & -.48202 & .71731 & .416 & 16 & .683 \\
\hline
\end{tabular}




\begin{tabular}{|c|c|}
\hline SET1 & SET2 \\
\hline \multicolumn{2}{|c|}{ 6. Please list 2 or 3 specific things this teacher did that stimulated and/or helped you to learn. } \\
\hline $\begin{array}{l}\text { Organising group-work for discussion (6), } \\
\text { organising interesting activities such as video- } \\
\text { recordings, games, storytelling (17) } \\
\text { encouraging interest in speaking (5), friendly } \\
\text { and enthusiastic teacher (3), organising } \\
\text { practical and logical activities (5) using visual } \\
\text { aids (2), clear instruction (7), giving bonus } \\
\text { grades (2) }\end{array}$ & $\begin{array}{l}\text { Organising interesting activities such as } \\
\text { storytelling, games, English songs, video clips } \\
\text { (14 ideas), cheerful, friendly and enthusiastic } \\
\text { teacher (5), clear instruction (2), good } \\
\text { pronunciation (1), encouraging students to } \\
\text { speak (5), praising students (2), organising } \\
\text { group-work (1), giving exercises (2), giving } \\
\text { bonus grades (2) }\end{array}$ \\
\hline \multicolumn{2}{|c|}{ 7. Please list 2 or 3 specific things this teacher might do to improve learning. } \\
\hline $\begin{array}{l}\text { Organising group-work for discussion (6), } \\
\text { encouraging competition and games among } \\
\text { groups (4), providing more materials (6), } \\
\text { developing listening and speaking skills, } \\
\text { vocabulary and grammar (4), using videos (1) }\end{array}$ & $\begin{array}{l}\text { Giving group-work exercises (8), pronunciation } \\
\text { correction (4), organising interesting activities } \\
\text { such as games, guessing words from pictures, } \\
\text { video clips (3), giving slower and detailed } \\
\text { presentations (e.g., examples for new words) } \\
\text { (4) revision (1), providing more materials (1), } \\
\text { guiding learning methods (3) }\end{array}$ \\
\hline
\end{tabular}

Khoa received mostly positive comments from her peer, which appeared to be the same for both POTs. However, suggests were given on speech flow and explanation of vocabulary, and developing reading skills (see table N5.2).

Table N5.2: Khoa's peer feedback

\begin{tabular}{|l|l|l|}
\hline & \multicolumn{1}{|c|}{ POT l feedback } & \multicolumn{1}{c|}{ POT2 feedback } \\
\hline aspects & $\begin{array}{l}\text { - Giving question-response tasks and } \\
\text { facilitating group/pair-work } \\
\text { presentations Singing: creating an } \\
\text { interesting atmosphere }\end{array}$ & $\begin{array}{l}\text { - Varied teaching organisation with } \\
\text { interesting activities via group/pair- } \\
\text { work presentations, games, video } \\
\text { clips }\end{array}$ \\
& $\begin{array}{l}\text { - Encouraging students to use English } \\
\text { to share ideas in familiar practical } \\
\text { topics Logical presentation }\end{array}$ & $\begin{array}{l}\text { - Logical delivery with sustainable } \\
\text { interactions because the students } \\
\text { were encouraged to communicate }\end{array}$ \\
& $\begin{array}{l}\text { - Being energetic and humorous } \\
\text { - Having an interesting way for lead-in }\end{array}$ & $\begin{array}{l}\text { Interesting introduction and lead-in } \\
\text { with audio-visual aids }\end{array}$ \\
& $\begin{array}{l}\text { Associating classroom language to } \\
\text { real-life context, and good } \\
\text { preparation, e.g. interesting logical } \\
\text { introduction }\end{array}$ \\
\hline $\begin{array}{l}\text { Aspects for } \\
\text { attention }\end{array}$ & $\begin{array}{l}\text { - Fast speech flow } \\
\text { - Explanation of word meaning }\end{array}$ & $\begin{array}{l}\text { Lack focus on language skills } \\
\text { Suggestions }\end{array}$ \\
$\begin{array}{l}\text { - Slowing down some sections } \\
\text { - Should use Vietnamese for } \\
\text { explaining abstract vocabulary: more } \\
\text { effective }\end{array}$ & $\begin{array}{l}\text { - For reading skills, it is necessary to } \\
\text { lead students' focus on the skills } \\
\text { such as skimming, scanning, and } \\
\text { identifying key words. }\end{array}$ \\
\hline
\end{tabular}

\section{Appendix N.6 Cuc's results of TT-SET and peer's feedback}

Student evaluation of Cuc's teaching before and after POT was similar because the differences in early-term and end-of-term rating means were not significant for most of Cuc's selected feedback items (Table N6.1) . 
Table N6.1: Difference in early-term and end-of-term TT-SETs for Cuc

\begin{tabular}{|c|c|c|c|c|c|c|c|c|c|c|}
\hline \multirow{3}{*}{ Feedback items } & \multirow{2}{*}{\multicolumn{2}{|c|}{ Mean }} & \multicolumn{5}{|c|}{ Paired Differences } & \multirow[b]{3}{*}{$t$} & \multirow[b]{3}{*}{ df } & \multirow{3}{*}{ 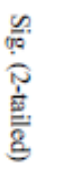 } \\
\hline & & & \multirow[b]{2}{*}{ Mean } & \multirow{2}{*}{$\begin{array}{c}\text { Std. } \\
\text { Deviation }\end{array}$} & \multirow{2}{*}{$\begin{array}{l}\text { Std. } \\
\text { Error } \\
\text { Mean }\end{array}$} & \multicolumn{2}{|c|}{$\begin{array}{l}95 \% \text { Confidence } \\
\text { Interval of the } \\
\text { Difference }\end{array}$} & & & \\
\hline & SET1 & SET2 & & & & Lower & Upper & & & \\
\hline $\begin{array}{l}\text { Q1. Teaching } \\
\text { organisation }\end{array}$ & 4.3793 & 4.3103 & -.06897 & 1.03272 & .19177 & -.46179 & .32386 & -.360 & 28 & .722 \\
\hline $\begin{array}{l}\text { Q2. Clarity of } \\
\text { communication }\end{array}$ & 4.4000 & 4.4000 & 0.00000 & 1.05045 & .19179 & -39225 & .39225 & 0.000 & 29 & 1.000 \\
\hline $\begin{array}{l}\text { Q3. Stimulation } \\
\text { of interest in } \\
\text { learning }\end{array}$ & 4.3667 & 4.3333 & -.03333 & .85029 & .15524 & -35084 & .28417 & -.215 & 29 & .831 \\
\hline $\begin{array}{l}\text { Q21. } \\
\text { Encouragement } \\
\text { of critical } \\
\text { thinking }\end{array}$ & 4.0769 & 4.4615 & .38462 & .98293 & .19277 & -.01240 & .78163 & 1.995 & 25 & .057 \\
\hline $\begin{array}{l}\text { Q25. } \\
\text { Encouragement } \\
\text { of creative } \\
\text { thinking }\end{array}$ & 4.2692 & 4.3077 & .03846 & 1.18257 & .23192 & -.43919 & .51611 & .166 & 25 & .870 \\
\hline Q30. Rapport & 4.4231 & 4.6923 & .26923 & .96157 & .18858 & -.11916 & .65762 & 1.428 & 25 & .166 \\
\hline $\begin{array}{l}\text { Q31. } \\
\text { Encouragement } \\
\text { of discussions }\end{array}$ & 4.3462 & 4.6538 & .30769 & 1.04954 & .20583 & -.11623 & .73161 & 1.495 & 25 & .147 \\
\hline $\begin{array}{l}\text { Q32. } \\
\text { Encouragement } \\
\text { of questioning }\end{array}$ & 4.2593 & 4.3704 & .11111 & 1.12090 & .21572 & -33230 & .55452 & .515 & 26 & .611 \\
\hline $\begin{array}{l}\text { Q33. } \\
\text { Encouragement } \\
\text { of idea and } \\
\text { knowledge } \\
\text { sharing }\end{array}$ & 4.4074 & 4.4444 & .03704 & 1.09128 & .21002 & -39466 & .46873 & .176 & 26 & .861 \\
\hline $\begin{array}{l}\text { Q37. } \\
\text { Appropriateness } \\
\text { of learning } \\
\text { evaluation }\end{array}$ & 4.2963 & 4.5556 & .25926 & .94432 & .18173 & -.11430 & .63282 & 1.427 & 26 & .166 \\
\hline
\end{tabular}

\begin{tabular}{|c|c|}
\hline SET1 & SET2 \\
\hline \multicolumn{2}{|c|}{ 6. Please list 2 or 3 specific things this teacher did that stimulated and/or helped you to learn. } \\
\hline $\begin{array}{l}\text { Organising interesting activities such as games, } \\
\text { English songs (24), developing language skills } \\
\text { (4), creating an environment for using English } \\
\text { with interesting situations (8), showing humour } \\
\text { friendliness, and rapport (8), clear instruction } \\
\text { (1), giving bonus grades (4), providing more } \\
\text { materials (5) }\end{array}$ & $\begin{array}{l}\text { Developing language skills (10), group-work } \\
\text { (5), interesting activities such as games and } \\
\text { songs (12), respecting students 'opinions (4), } \\
\text { giving bonus grades (7), clear instruction and } \\
\text { voice (5), showing enthusiasm and care for } \\
\text { students (2), practical content (1), promoting } \\
\text { self-study (1), giving homework (1), creating } \\
\text { active and comfortable atmosphere (6), } \\
\text { introducing the importance of the subject (1) }\end{array}$ \\
\hline & ght do to improve learning. \\
\hline $\begin{array}{l}\text { Providing more materials (6), developing } \\
\text { language skills (5), giving clearer instruction } \\
\text { (2), using Vietnamese for difficult terms (2), } \\
\text { using only English (1), more group-work (2), no } \\
\text { midterm (1), creating more communication } \\
\text { situations (1), guiding learning methods (1), fun } \\
\text { activities such as games and songs (6) }\end{array}$ & $\begin{array}{l}\text { Developing language skills more (13), } \\
\text { organising games and songs (2), encouraging } \\
\text { more group-work discussion (5), providing } \\
\text { more exercises and knowledge in laws (8), } \\
\text { using only English (1), guiding learning } \\
\text { methods (3), correcting students' use of } \\
\text { language (1), assignments (1), using class time } \\
\text { effectively }\end{array}$ \\
\hline
\end{tabular}

Like student feedback, Cuc's peer mentioned similar strengths after observing Cuc teach two times and did not give any suggestions for changes as shown in Table N6.2. 
Table N6.2: Cuc's peer feedback

\begin{tabular}{|l|l|l|}
\hline & \multicolumn{1}{|c|}{ POT 1 feedback } & \multicolumn{1}{c|}{ POT2 feedback } \\
\hline $\begin{array}{l}\text { Good } \\
\text { aspects }\end{array}$ & $\begin{array}{l}\text { - Students being enthusiastic and } \\
\text { engaged in activities } \\
\text { - Varied teacher-student and student- } \\
\text { student interactions, practice on } \\
\text { different skills with group-work in } \\
\text { listening, speaking } \\
\text { - Integrated four language skills } \\
\text { - Offering rapport and support to each } \\
\text { group }\end{array}$ & $\begin{array}{l}\text { - Interesting organisation of activities } \\
\text { students a language environment for } \\
\text { - Integrating four language skills } \\
\text { - Promoting independent thinking and } \\
\text { method of self-study }\end{array}$ \\
\hline $\begin{array}{l}\text { Aspects for } \\
\text { attention }\end{array}$ & - No opinion & - No opinion \\
\hline Suggestions & - No opinion & - No opinion \\
\hline
\end{tabular}

\section{Appendix N.7 Giang's results of TT-SET and peer's feedback}

Table N7.1 shows that there were significant differences with three items being rated higher while the other aspects remained unchanged.

Table N7.1: Differences in early-term and end-of-term TT-SETs for Giang

\begin{tabular}{|c|c|c|c|c|c|c|c|c|c|c|}
\hline \multirow[b]{3}{*}{ Feedback items } & \multirow{2}{*}{\multicolumn{2}{|c|}{ Mean }} & \multicolumn{5}{|c|}{ Paired Differences } & \multirow[b]{3}{*}{$\mathrm{t}$} & \multirow[b]{3}{*}{ df } & \multirow{3}{*}{ 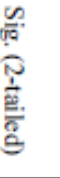 } \\
\hline & & & \multirow[b]{2}{*}{ Mean } & \multirow{2}{*}{$\begin{array}{c}\text { Std. } \\
\text { Deviation }\end{array}$} & \multirow{2}{*}{$\begin{array}{l}\text { Std. } \\
\text { Error } \\
\text { Mean }\end{array}$} & \multicolumn{2}{|c|}{$\begin{array}{l}95 \% \text { Confidence } \\
\text { Interval of the } \\
\text { Difference }\end{array}$} & & & \\
\hline & SET1 & SET2 & & & & Lower & Upper & & & \\
\hline $\begin{array}{l}\text { Q1. Teaching } \\
\text { organisation }\end{array}$ & 3.8824 & 4.4118 & .52941 & 1.02204 & .17528 & -88602 & -17281 & 3.020 & 33 & .005 \\
\hline $\begin{array}{l}\text { Q2. Clarity of } \\
\text { communication }\end{array}$ & 4.0909 & 4.5758 & .48485 & 90558 & .15764 & -80595 & -.16374 & 3.076 & 32 & .004 \\
\hline $\begin{array}{l}\text { Q20. } \\
\text { Constructive } \\
\text { individual help }\end{array}$ & 4.2121 & 4.6364 & .42424 & .86712 & .15095 & -.73171 & -11678 & 2.811 & 32 & .008 \\
\hline $\begin{array}{l}\text { Q21. } \\
\text { Intellectual } \\
\text { challenging } \\
\text { materials }\end{array}$ & 4.1143 & 4.3429 & .22857 & 84316 & .14252 & -.51821 & .06107 & 1.604 & 34 & .118 \\
\hline $\begin{array}{l}\text { Q23. } \\
\text { Encouragement } \\
\text { of class } \\
\text { discussions }\end{array}$ & 4.4286 & 4.5429 & .11429 & .58266 & .09849 & -31444 & .08587 & 1.160 & 34 & .254 \\
\hline $\begin{array}{l}\text { Q25. } \\
\text { Encouragement } \\
\text { of idea and } \\
\text { knowledge } \\
\text { sharing }\end{array}$ & 4.4706 & 4.4118 & -.05882 & .64860 & .11123 & -16748 & .28513 & -.529 & 33 & .600 \\
\hline
\end{tabular}




\begin{tabular}{|c|c|}
\hline SET1 & SET2 \\
\hline \multicolumn{2}{|c|}{ 6. Please list 2 or 3 specific things this teacher did that stimulated and/or helped you to learn. } \\
\hline $\begin{array}{l}\text { Organising interesting activities such as games, } \\
\text { storytelling with humour (25), clear instruction } \\
\text { that stimulate learning (4), giving bonus grades } \\
\text { (4), providing good materials (1), visual aids } \\
\text { (1), using English much (1), lovely smiles (1), } \\
\text { practical content and good materials (5), } \\
\text { encouraging creativity (1), developing speaking } \\
\text { skills and vocabulary (2), responding to } \\
\text { students' feedback (1), group-work (2) }\end{array}$ & $\begin{array}{l}\text { Interesting activities such as games, } \\
\text { storytelling, and songs (34), group-work } \\
\text { presentations (15), humour and enthusiasm } \\
\text { (16), interesting Powerpoint slides (3), being } \\
\text { beautiful (1), good examples and knowledge } \\
\text { (3), guiding learning methods and taking exams } \\
\text { (2) }\end{array}$ \\
\hline \multicolumn{2}{|c|}{ 7. Please list 2 or 3 specific things this teacher might do to improve learning. } \\
\hline $\begin{array}{l}\text { More group-work (4), developing speaking and } \\
\text { listening skills with vocabulary (5), giving } \\
\text { homework (3), bonus grades (1), more materials } \\
\text { (1), outdoor activities, songs, and games (3), } \\
\text { responding to students' questions (2), giving } \\
\text { individual care (1), delivering content } \\
\text { appropriate for students' level (1) }\end{array}$ & $\begin{array}{l}\text { Giving more exercises and practice (6), } \\
\text { developing listening and speaking skills with } \\
\text { vocabulary (5), clearer instruction on } \\
\text { vocabulary (2), encouraging discussion and } \\
\text { presentations (3), more games and songs (5), } \\
\text { using translation (2), encouraging students to } \\
\text { speak (1) }\end{array}$ \\
\hline
\end{tabular}

Giang's peer also detected her improvement in organisation of group work and clarity of communicating information and ideas, as shown in Table N7.2.

Table N7.2: Giang's peer feedback

\begin{tabular}{|c|c|c|}
\hline & POT 1 feedback & POT2 feedback \\
\hline $\begin{array}{l}\text { Good } \\
\text { aspects }\end{array}$ & $\begin{array}{l}\text { - Intellectually challenging content, } \\
\text { which required critical thinking } \\
\text { - Organising group presentations and } \\
\text { teaching grammar via games, which } \\
\text { was good }\end{array}$ & $\begin{array}{l}\text { - Presenting the lesson clearly and } \\
\text { logically } \\
\text { - Facilitating group presentation in } \\
\text { each session } \\
\text { - Giving a chance to each student to } \\
\text { practice } \\
\text { - Organising group-work with reading } \\
\text { activities, which was good } \\
\text { - Giving clear feedback and correcting } \\
\text { students' pronunciation and speaking } \\
\text { or presentation skills } \\
\text { - Good way to organise group-work } \\
\text { and apply IT in teaching such as } \\
\text { designing beautiful slideshows }\end{array}$ \\
\hline $\begin{array}{l}\text { Aspects for } \\
\text { attention }\end{array}$ & $\begin{array}{l}\text { - Students being not engaged } \\
\text { - Unclear communication of ideas }\end{array}$ & $\begin{array}{l}\text { - Problem of time control } \\
\text { - inappropriate level for listening task }\end{array}$ \\
\hline Suggestions & $\begin{array}{l}\text { - Learners finding it difficult to follow } \\
\text { the lesson due to interruptions } \\
\text { - Giving clearer explanation on } \\
\text { grammatical points } \\
\text { - Avoid distraction from technical } \\
\text { devices which hindered students' } \\
\text { attention } \\
\text { - Paying attention to voice because } \\
\text { high-pitched voice was hard for } \\
\text { students when the academic was too } \\
\text { enthusiastic } \\
\text { - Should use more English in the } \\
\text { classroom } \\
\text { - Necessary to spend time revising } \\
\text { theoretical knowledge }\end{array}$ & $\begin{array}{l}\text { - Adjusting listening tasks to match } \\
\text { the student level because the } \\
\text { listening task of the class seemed to } \\
\text { be a lot difficult for the students } \\
\text { - Paying attention to time control } \\
\text { because it was a problem and time } \\
\text { schedule was not strictly followed }\end{array}$ \\
\hline
\end{tabular}




\section{Appendix N.8 Nga's results of TT-SET and peer's feedback}

Table N8.1 shows that the differences were not significant for both TT-SET results.

Table 8.1: Difference in early-term and end-of-term TT-SETs for Nga

\begin{tabular}{|c|c|c|c|c|c|c|c|c|c|c|}
\hline \multirow{3}{*}{ Feedback items } & & & \multicolumn{5}{|c|}{ Paired Differences } & \multirow{3}{*}{$\mathrm{t}$} & \multirow{3}{*}{$\mathrm{df}$} & \multirow{3}{*}{ 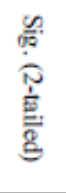 } \\
\hline & \multicolumn{2}{|c|}{ Mean } & \multirow[t]{2}{*}{ Mean } & \multirow[t]{2}{*}{$\begin{array}{c}\text { Std. } \\
\text { Deviation }\end{array}$} & \multirow[t]{2}{*}{$\begin{array}{l}\text { Std. } \\
\text { Error } \\
\text { Mean }\end{array}$} & \multicolumn{2}{|c|}{$\begin{array}{l}95 \% \text { Confidence } \\
\text { Interval of the } \\
\text { Difference }\end{array}$} & & & \\
\hline & SET1 & SET2 & & & & Lower & Upper & & & \\
\hline $\begin{array}{l}\text { Q1. Teaching } \\
\text { organisation }\end{array}$ & 3.9167 & 4.5000 & -.58333 & .99620 & .28758 & $-121,629$ & .04963 & $-2,028$ & 11 & .067 \\
\hline $\begin{array}{l}\text { Q3. Stimulation } \\
\text { of interest in } \\
\text { learning }\end{array}$ & 4.4167 & 4.4167 & .00000 & 127,920 & .36927 & -81277 & .81277 & .000 & 11 & 1.000 \\
\hline $\begin{array}{l}\text { Q8. Delivery } \\
\text { holding interest }\end{array}$ & 4.1538 & 4.3846 & -.23077 & 109,193 & .30285 & -89061 & .42908 & -.762 & 12 & .461 \\
\hline $\begin{array}{l}\text { Q9. } \\
\text { Encouragement } \\
\text { of critical } \\
\text { thinking }\end{array}$ & 3.7692 & 4.2308 & -.46154 & 119,829 & .33235 & $-118,566$ & .26258 & $-1,389$ & 12 & .190 \\
\hline $\begin{array}{l}\text { Q10. Work set } \\
\text { to encourage } \\
\text { learning }\end{array}$ & 4.2308 & 4.0769 & .15385 & 106,819 & .29626 & -49165 & .79935 & .519 & 12 & .613 \\
\hline $\begin{array}{l}\text { Q11. } \\
\text { Encouragement } \\
\text { of discussions }\end{array}$ & 4.6923 & 4.6154 & .07692 & .75955 & .21066 & -38207 & .53592 & .365 & 12 & .721 \\
\hline
\end{tabular}

\section{SET1}

SET2

6. Please list 2 or 3 specific things this teacher did that stimulated and/or helped you to learn. Organising interesting activities such as games, $\quad$ Interesting activities such as games (8), competition, and songs (20), group-work discussion and presentations (10), checking assignments (9), content appropriate for students (3), clear instruction (4), showing enthusiasm, encouragement and support (6), appropriate way of evaluating students (4), giving bonus grades (3) friendliness, enthusiasm, and empathy (5), clear, practical and interesting instruction (4), promoting interest in learning (7), creating an environment for language use (1), checking lesson preparation (1), regular revision of lessons (2), providing deep knowledge (2), giving bonus grades (1)

7. Please list 2 or 3 specific things this teacher might do to improve learning.

Checking assignments (8), more games and friendlier atmosphere (10), group-work (3), individual care (4), developing language skills with pronunciation (5), providing more economic vocabulary and knowledge (4), providing hand-outs and quizzes (2), delivering content more appropriate to students (1), creating more comfortable atmosphere (4) Creating an environment for language use (3), giving assignments (4), guiding how to memorise vocabulary (3), clearer instruction (1), encouraging self-study (1), using visual aids (1), giving slower instruction (1), translation (1), sample tests (1)

Nga received just positive feedback from her peer for both POTs without any suggestions for changes, as shown in Table N8.2. 
Table N8.2: Nga's peer feedback

\begin{tabular}{|c|c|c|}
\hline & POT 1 feedback & POT2 feedback \\
\hline $\begin{array}{l}\text { Good } \\
\text { aspects }\end{array}$ & $\begin{array}{l}\text { - Using games for warm-up, using } \\
\text { gestures and even Vietnamese to } \\
\text { explain difficult vocabulary, making } \\
\text { jokes to help the students relax, } \\
\text { giving "punishments" to encourage } \\
\text { participation } \\
\text { - Introducing vocabulary via guessing } \\
\text { games, explanations, synonyms, } \\
\text { antonyms, or translation } \\
\text { - Being energetic and enthusiastic to } \\
\text { create an active atmosphere }\end{array}$ & $\begin{array}{l}\text { - Students being more excited and } \\
\text { happy with games and the academic } \\
\text { remembering their names } \\
\text { - Setting group presentation so that } \\
\text { students could help and encourage } \\
\text { each other } \\
\text { - Designing some more exercises to } \\
\text { help students grasp the lesson better } \\
\text { - Initiating a lesson review by a } \\
\text { question-response task }\end{array}$ \\
\hline $\begin{array}{l}\text { Aspects for } \\
\text { attention }\end{array}$ & - No opinion & - No opinion \\
\hline Suggestions & - No opinion & - No opinion \\
\hline
\end{tabular}

\section{Appendix N.9 Binh's results of TT-SET and peer's feedback}

Table N9.1 shows that the differences in TT-SET results were not significant.

Table N9.1: Difference in early-term and end-of-term TT-SETs for Binh

\begin{tabular}{|c|c|c|c|c|c|c|c|c|c|c|}
\hline \multirow{3}{*}{ Feedback items } & \multirow{2}{*}{\multicolumn{2}{|c|}{ Mean }} & \multicolumn{5}{|c|}{ Paired Differences } & \multirow[b]{3}{*}{$\mathrm{t}$} & \multirow[b]{3}{*}{ df } & $\stackrel{n}{\varphi x}$ \\
\hline & & & \multirow[b]{2}{*}{ Mean } & \multirow{2}{*}{$\begin{array}{c}\text { Std. } \\
\text { Deviation }\end{array}$} & \multirow{2}{*}{$\begin{array}{l}\text { Std. } \\
\text { Error } \\
\text { Mean }\end{array}$} & \multicolumn{2}{|c|}{$\begin{array}{l}95 \% \text { Confidence } \\
\text { Interval of the } \\
\text { Difference }\end{array}$} & & & 良 \\
\hline & SET1 & SET2 & & & & Lower & Upper & & & \\
\hline $\begin{array}{l}\text { Q1. Teaching } \\
\text { organisation }\end{array}$ & 3.800 & 3.850 & .05000 & 1.19097 & .26631 & -.50739 & .60739 & .188 & 19 & .853 \\
\hline $\begin{array}{l}\text { Q2. Clarity of } \\
\text { communication }\end{array}$ & 4.318 & 4.318 & 0.00000 & .75593 & .16116 & -33516 & .33516 & 0.000 & 21 & 1.000 \\
\hline $\begin{array}{l}\text { Q3. Stimulation of } \\
\text { interest in learning }\end{array}$ & 4.091 & 3.864 & -.22727 & 1.06600 & .22727 & -69991 & .24537 & -1.000 & 21 & .329 \\
\hline $\begin{array}{l}\text { Q11. Delivery } \\
\text { holding interest }\end{array}$ & 3.900 & 3.950 & .05000 & 1.09904 & .24575 & -.46437 & .56437 & .203 & 19 & .841 \\
\hline $\begin{array}{l}\text { Q19. } \\
\text { Encouragement of } \\
\text { discussions }\end{array}$ & 4.524 & 4.476 & -.04762 & .74001 & .16148 & -.38447 & .28923 & -.295 & 20 & .771 \\
\hline $\begin{array}{l}\text { Q20. } \\
\text { Encouragement of } \\
\text { questioning }\end{array}$ & 4.476 & 4.429 & -.04762 & .58959 & .12866 & -31600 & .22076 & -.370 & 20 & .715 \\
\hline $\begin{array}{l}\text { Q21. } \\
\text { Encouragement of } \\
\text { idea and } \\
\text { knowledge } \\
\text { sharing }\end{array}$ & 4.476 & 4.286 & -.19048 & .60159 & .13128 & -46431 & .08336 & -1.451 & 20 & .162 \\
\hline $\begin{array}{l}\text { Q23. Contribution } \\
\text { of readings, } \\
\text { homework, etc. to } \\
\text { appreciation and } \\
\text { understanding }\end{array}$ & 4.000 & 3.762 & -.23810 & .99523 & .21718 & -.69112 & .21493 & -1.096 & 20 & 286 \\
\hline
\end{tabular}


6. Please list 2 or 3 specific things this teacher did that stimulated and/or helped you to learn. Organising interesting activities such as games, $\quad$ Organising interesting activities such as games, storytelling (12), friendly and enthusiastic teacher (8), respecting students' opinions (3), organising group-work (2), encouraging students to speak (1), providing interesting materials (1), giving bonus grades (2) English songs (30 ideas), giving exercises (7), organising group-work for using English (6), developing language skills and vocabulary (3), giving interesting topics for presentations (1), creating a comfortable atmosphere with support (2), teacher having good look (1), encouraging students to give opinions (2)

7. Please list 2 or 3 specific things this teacher might do to improve learning. Providing more exercises and materials (7), developing speaking and listening skills, vocabulary and grammar (9), guiding learning methods (1), being more enthusiastic (1), using Vietnamese to explain difficult terms (1), listening to students' feedback (1)

Providing materials, exercises, video clips (11),
organising interesting activities such as games,
English songs (14), using Vietnamese (1),
sample tests (1), guiding learning methods and
doing tests (4), giving clear instruction (3),
developing language skills and vocabulary
(13), assigning students' presentations (5)

Binh's peers (An and Cuc) identified different strengths and weaknesses regarding her practice during two observations. An did not return the peer observer's written feedback but discussed it with Binh at the debriefing on issues such as inappropriate timing (e.g., long introduction), failure to engage students, unclear presentation, speaking too fast, nervousness from being observed (An-DEB) but improved practice after POT2 (RJ). Cuc's comments is shown in Table N9.2.

Table N9.2: Binh's peer feedback (from Cuc)

\begin{tabular}{|c|c|c|}
\hline & POT 1 feedback & POT2 feedback \\
\hline $\begin{array}{l}\text { Good } \\
\text { aspects }\end{array}$ & $\begin{array}{l}\text { - Interweaving communication } \\
\text { activities interwoven into lesson } \\
\text { content like grammar } \\
\text { - Clear, understandable, and } \\
\text { interesting presentation }\end{array}$ & $\begin{array}{l}\text { - Good method with dynamism and } \\
\text { flexibility }\end{array}$ \\
\hline $\begin{array}{l}\text { Aspects for } \\
\text { attention }\end{array}$ & $\begin{array}{l}\text { - Class management: too much time } \\
\text { for grouping }\end{array}$ & $\begin{array}{l}\text { - "One-way" interaction for student } \\
\text { communication activities like, set by } \\
\text { the academic rather than self- } \\
\text { awareness. } \\
\text { - Passive students } \\
\text { - Much dead time }\end{array}$ \\
\hline Suggestions & $\begin{array}{l}\text { - Should reconsider organising group } \\
\text { or pair-work discussions } \\
\text { - More dynamical to encourage } \\
\text { passive students to join in } \\
\text { communication }\end{array}$ & $\begin{array}{l}\text { - Should state the purpose of activities } \\
\text { such as what they learned from the } \\
\text { instruction, which skills, whether } \\
\text { they could use those skills in the } \\
\text { future. } \\
\text { - Making students more active and } \\
\text { promoting their self-study ability }\end{array}$ \\
\hline
\end{tabular}

\section{Appendix N.10 Phung's results of TT-SET and peer's feedback}

Table N10.1 shows that the differences between rating means of early-term and endof-term TT-SETs were significant with three items being rated higher. 
Table N10.1: Differences in early-term and end-of-term TT-SETs for Phung

\begin{tabular}{|c|c|c|c|c|c|c|c|c|c|c|}
\hline \multirow{3}{*}{ Questions } & \multirow{2}{*}{\multicolumn{2}{|c|}{ Mean }} & \multicolumn{5}{|c|}{ Paired Differences } & \multirow[b]{3}{*}{$t$} & \multirow[b]{3}{*}{ df } & \multirow{3}{*}{ 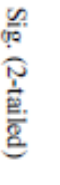 } \\
\hline & & & \multirow[b]{2}{*}{ Mean } & \multirow{2}{*}{$\begin{array}{c}\text { Std. } \\
\text { Deviation }\end{array}$} & \multirow{2}{*}{$\begin{array}{l}\text { Std. } \\
\text { Error } \\
\text { Mean }\end{array}$} & \multicolumn{2}{|c|}{$\begin{array}{l}95 \% \text { Confidence } \\
\text { Interval of the } \\
\text { Difference }\end{array}$} & & & \\
\hline & SET1 & SET2 & & & & Lower & Upper & & & \\
\hline $\begin{array}{l}\text { Q3. Stimulation } \\
\text { of interest in } \\
\text { learning }\end{array}$ & 3.3846 & 4.1538 & .76923 & 90808 & .17809 & .40245 & $\begin{array}{r}1.1360 \\
1\end{array}$ & 4.319 & 25 & .000 \\
\hline $\begin{array}{l}\text { Q10. Use of } \\
\text { humour }\end{array}$ & 3.4231 & 4.1154 & .69231 & 1.12318 & .22027 & .23864 & $\begin{array}{r}1.1459 \\
7 \\
\end{array}$ & 3.143 & 25 & .004 \\
\hline $\begin{array}{l}\text { Q18. Delivery at } \\
\text { an appropriate } \\
\text { level }\end{array}$ & 3.6667 & 4.1111 & .44444 & 1.08604 & .20901 & .01482 & .87407 & 2.126 & 26 & .043 \\
\hline $\begin{array}{l}\text { Q19. } \\
\text { Constructive } \\
\text { individual help }\end{array}$ & 4.2593 & 4.0741 & -.18519 & 1.17791 & .22669 & -65115 & .28078 & -.817 & 26 & .421 \\
\hline Q21. Rapport & 4.2000 & 4.4400 & .24000 & 1.01160 & .20232 & -17757 & .65757 & 1.186 & 24 & 247 \\
\hline $\begin{array}{l}\text { Q22. } \\
\text { Encouragement } \\
\text { of discussions }\end{array}$ & 4.3846 & 4.5385 & .15385 & .73170 & .14350 & -14169 & .44939 & 1.072 & 25 & .294 \\
\hline $\begin{array}{l}\text { Q23. } \\
\text { Encouragement } \\
\text { of questioning }\end{array}$ & 4.4231 & 4.5385 & .11538 & .71144 & .13953 & -17197 & .40274 & .827 & 25 & 416 \\
\hline $\begin{array}{l}\text { Q24. } \\
\text { Encouragement } \\
\text { of idea and } \\
\text { knowledge } \\
\text { sharing }\end{array}$ & 4.3333 & 4.4074 & .07407 & 1.07152 & .20621 & -34980 & .49795 & .359 & 26 & .722 \\
\hline $\begin{array}{l}\text { Q27. } \\
\text { Appropriateness } \\
\text { of learning } \\
\text { evaluation }\end{array}$ & 4.1667 & 4.4583 & 29167 & .75060 & .15322 & -.02529 & .60862 & 1.904 & 23 & .070 \\
\hline
\end{tabular}

SET1

SET2

6. Please list 2 or 3 specific things this teacher did that stimulated and/or helped you to learn. Showing care for students and encouragement (9), strict but friendly and enthusiastic teacher (4), developing language skills with pronunciation and vocabulary (9), deep knowledge (3), introducing the importance of the subject (1), giving bonus grades (3), being humorous (5), organising group-work for discussion or presentations (5), clear instruction (5), organising games (1) Organising interesting activities such as games, English songs, storytelling, videos (32 ideas), giving exercises (7), clear instruction (1), promoting interest and confidence (3), revision and quizzes (3), organising group-work and presentations (7), attractive voice (1), respecting students' opinions (2), providing materials (1), developing vocabulary (1), giving bonus grades (2)

7. Please list 2 or 3 specific things this teacher might do to improve learning.

Providing more materials and exercises (14), encouraging students to use English (5), developing listening and speaking skills with pronunciation (7), sharing experience (2), organising group-work (5), guiding learning methods (2), organising games (4), giving slow and detailed instruction (6), giving homework (6), being friendlier (4) Providing more materials such as exercises, songs, video clips (20), organising group-work for discussion and presentations (10), developing language skills and vocabulary (7), organising games (4), sharing experience (1), checking attendance (1), giving detailed instruction (2), enthusiasm (1), allowing break time (1)

Phung received peer similar feedback for both POTs as shown in Table N10.2. 
Table N10.2: Phung's peer feedback

\begin{tabular}{|c|c|c|}
\hline & POT 1 feedback & POT2 feedback \\
\hline $\begin{array}{l}\text { Good } \\
\text { aspects }\end{array}$ & $\begin{array}{l}\text { - Revising leadership styles and } \\
\text { introducing word collocation as } \\
\text { warm-up } \\
\text { - Encouraging students to express their } \\
\text { opinions Giving clear phonetic } \\
\text { transcriptions of vocabulary and } \\
\text { delivering lessons at appropriate } \\
\text { speed }\end{array}$ & $\begin{array}{l}\text { - Properly arranged activities for } \\
\text { teaching delivery } \\
\text { - Having good knowledge to lead in } \\
\text { the reading } \\
\text { - Selecting suitable content to the } \\
\text { learners } \\
\text { - Encouraging students to } \\
\text { communicate in English with } \\
\text { instruction on pronunciation } \\
\text { - Delivering the lesson at appropriate } \\
\text { speed }\end{array}$ \\
\hline $\begin{array}{l}\text { Aspects for } \\
\text { attention }\end{array}$ & $\begin{array}{l}\text { - Teacher-student interaction, } \\
\text { instructions, and feedback }\end{array}$ & $\begin{array}{l}\text { - Academic-student and student- } \\
\text { student interactions }\end{array}$ \\
\hline Suggestions & $\begin{array}{l}\text { - Avoid being so serious in class. } \\
\text { - Should have more interactions with } \\
\text { students by asking questions, which } \\
\text { could be a way to check whether the } \\
\text { lesson was at a level appropriate for } \\
\text { them [DEB] } \\
\text { - Better to give clear instructions for } \\
\text { each session and requiring students } \\
\text { to share ideas. } \\
\text { - Avoiding interfering much in } \\
\text { students' communication such as } \\
\text { after a presentation, students have to } \\
\text { ask their classmates questions and } \\
\text { get their opinions [DEB] }\end{array}$ & $\begin{array}{l}\text { - Necessary to have more academic- } \\
\text { student and student-student } \\
\text { interactions }\end{array}$ \\
\hline
\end{tabular}




\section{Appendix N.11 An's results of TT-SET and peer's feedback}

Table N11.1 shows that An received significantly lower ratings on seven teaching aspects.

Table N11.1: Difference in early-term and end-of-term TT-SETs for An

\begin{tabular}{|c|c|c|c|c|c|c|c|c|c|c|}
\hline \multirow[b]{3}{*}{ Feedback items } & & & \multicolumn{5}{|c|}{ Paired Differences } & \multirow[b]{3}{*}{$t$} & \multirow[b]{3}{*}{ df } & \multirow{3}{*}{ 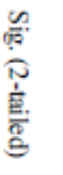 } \\
\hline & \multicolumn{2}{|c|}{ Mean } & \multirow[b]{2}{*}{ Mean } & \multirow{2}{*}{$\begin{array}{c}\text { Std. } \\
\text { Deviation }\end{array}$} & \multirow{2}{*}{$\begin{array}{l}\text { Std. } \\
\text { Error } \\
\text { Mean }\end{array}$} & \multicolumn{2}{|c|}{$\begin{array}{l}95 \% \text { Confidence } \\
\text { Interval of the } \\
\text { Difference }\end{array}$} & & & \\
\hline & SET1 & SET2 & & & & Lower & Upper & & & \\
\hline $\begin{array}{l}\text { Q1. Teaching } \\
\text { organisation }\end{array}$ & 3.1034 & 2.5862 & -.51724 & 1.32613 & .24626 & .01281 & 1.02167 & -2.100 & 28 & .045 \\
\hline $\begin{array}{l}\text { Q3. Stimulation of } \\
\text { interest in leaming }\end{array}$ & 2.9000 & 2.4667 & -.43333 & 1.40647 & .25679 & -.09185 & .95852 & -1.688 & 29 & .102 \\
\hline $\begin{array}{l}\text { Q8. Dynamism } \\
\text { and being } \\
\text { energetic }\end{array}$ & 3.6552 & 3.2414 & -.41379 & 1.21059 & .22480 & -.04669 & .87428 & -1.841 & 28 & .076 \\
\hline $\begin{array}{l}\text { Q9. Use of } \\
\text { humour }\end{array}$ & 3.7667 & 3.0667 & -.70000 & 1.39333 & .25439 & .17972 & 1.22028 & -2.752 & 29 & .010 \\
\hline $\begin{array}{l}\text { Q13. } \\
\text { Encouragement of } \\
\text { critical thinking }\end{array}$ & 3.7407 & 3.0741 & -.66667 & 1.07417 & .20672 & .24174 & 1.09160 & -3.225 & 26 & .003 \\
\hline $\begin{array}{l}\text { Q17. Useful } \\
\text { materials }\end{array}$ & 3.4828 & 3.0000 & -.48276 & 1.08958 & .20233 & .06830 & .89721 & -2.386 & 28 & .024 \\
\hline Q18. Rapport & 3.4828 & 2.7931 & -.68966 & 1.16813 & .21692 & .24532 & 1.13399 & -3.179 & 28 & .004 \\
\hline $\begin{array}{l}\text { Q19. } \\
\text { Encouragement of } \\
\text { discussions }\end{array}$ & 3.9667 & 3.1000 & -.86667 & .81931 & .14958 & .56073 & 1.17260 & -5.794 & 29 & .000 \\
\hline $\begin{array}{l}\text { Q21. Hand-outs as } \\
\text { valuable aids to } \\
\text { learning }\end{array}$ & 3.4815 & 2.9259 & -.55556 & 1.15470 & .22222 & .09877 & 1.01234 & -2.500 & 26 & .019 \\
\hline
\end{tabular}

SET1

SET2

6. Please list 2 or 3 specific things this teacher did that stimulated and/or helped you to learn. \begin{tabular}{l|l} 
Providing more exercises (9), interesting & Developing language skills (2), informing testing
\end{tabular} activities such as games and storytelling (9), $\quad$ schedule (2), checking assignments (1), giving encouraging students to speak (4), clear $\quad$ bonus grades (3), storytelling (4), providing explanations for exercises (1), encouraging exercises regularly (1), encouraging students to creativity (1), giving bonus grades (4), checking attendance (5), group-work (1) give opinions (1)

7. Please list 2 or 3 specific things this teacher might do to improve learning.

Developing listening skills with pronunciation $\quad$ Providing more materials (5), delivering more and communication in English (6), providing more useful materials and exercises (8), delivering more interesting lessons (2), checking assignments (1) interesting lessons with detailed explanations (3), guiding learning methods (3), delivering slower and clearer instruction (2), recommending websites to learn English (1), giving bonus grades (1), checking assignments (1)

An received just positive written feedback from her peer, as shown in Table N11.2. 
Table N11.2: An's peer feedback

\begin{tabular}{|c|c|c|}
\hline & POT 1 feedback & POT2 feedback \\
\hline $\begin{array}{l}\text { Good } \\
\text { aspects }\end{array}$ & $\begin{array}{l}\text { - Communicating ideas and } \\
\text { information clearly and in details } \\
\text { - Being humorous, enthusiastic, } \\
\text { supportive } \\
\text { - Moving around to support students } \\
\text { - Engaging students in learning }\end{array}$ & $\begin{array}{l}\text { - Good organisation of } \\
\text { - Asking students to give feedback on } \\
\text { their classmates' presentation }\end{array}$ \\
\hline $\begin{array}{l}\text { Aspects for } \\
\text { attention }\end{array}$ & - Not facilitating self-production & - Giving non-constructive feedback \\
\hline Suggestions & $\begin{array}{l}\text { - Should ask students to give more } \\
\text { examples of the grammatical } \\
\text { structure they learned }\end{array}$ & $\begin{array}{l}\text { - Should offer constructive feedback } \\
\text { and advice }\end{array}$ \\
\hline
\end{tabular}

